\title{
Global surveillance of trends in cancer survival 2000-14 (CONCORD-3): analysis of individual records for 37513025 patients diagnosed with one of 18 cancers from 322 population-based registries in 71 countries
}

\author{
Claudia Allemani, Tomohiro Matsuda, Veronica Di Carlo, Rhea Harewood, Melissa Matz, Maja Nikšić, Audrey Bonaventure, Mikhail Valkov, \\ Christopher JJohnson, Jacques Estève, Olufemi J Ogunbiyi, Gulnar Azevedo e Silva, Wan-Qing Chen, Sultan Eser, Gerda Engholm, Charles A Stiller, \\ Alain Monnereau, Ryan R Woods, Otto Visser, Gek Hsiang Lim, Joanne Aitken, Hannah K Weir, Michel P Coleman, CONCORD Working Group*
}

\section{Summary}

Background In 2015, the second cycle of the CONCORD programme established global surveillance of cancer survival as a metric of the effectiveness of health systems and to inform global policy on cancer control. CONCORD-3 updates the worldwide surveillance of cancer survival to 2014.

Methods CONCORD-3 includes individual records for 37.5 million patients diagnosed with cancer during the 15-year period 2000-14. Data were provided by 322 population-based cancer registries in 71 countries and territories, 47 of which provided data with $100 \%$ population coverage. The study includes 18 cancers or groups of cancers: oesophagus, stomach, colon, rectum, liver, pancreas, lung, breast (women), cervix, ovary, prostate, and melanoma of the skin in adults, and brain tumours, leukaemias, and lymphomas in both adults and children. Standardised quality control procedures were applied; errors were rectified by the registry concerned. We estimated 5-year net survival. Estimates were age-standardised with the International Cancer Survival Standard weights.

Findings For most cancers, 5-year net survival remains among the highest in the world in the USA and Canada, in Australia and New Zealand, and in Finland, Iceland, Norway, and Sweden. For many cancers, Denmark is closing the survival gap with the other Nordic countries. Survival trends are generally increasing, even for some of the more lethal cancers: in some countries, survival has increased by up to $5 \%$ for cancers of the liver, pancreas, and lung. For women diagnosed during 2010-14, 5-year survival for breast cancer is now $89.5 \%$ in Australia and $\mathbf{9 0 \cdot 2 \%}$ in the USA, but international differences remain very wide, with levels as low as $66 \cdot 1 \%$ in India. For gastrointestinal cancers, the highest levels of 5-year survival are seen in southeast Asia: in South Korea for cancers of the stomach $(68.9 \%)$, colon $(71.8 \%)$, and rectum (71.1\%); in Japan for oesophageal cancer (36.0\%); and in Taiwan for liver cancer (27.9\%). By contrast, in the same world region, survival is generally lower than elsewhere for melanoma of the skin $\mathbf{5 9 . 9 \%}$ in South Korea, $52 \cdot 1 \%$ in Taiwan, and $49.6 \%$ in China), and for both lymphoid malignancies $(52 \cdot 5 \%, 50 \cdot 5 \%$, and $38 \cdot 3 \%$ ) and myeloid malignancies $(45 \cdot 9 \%$, $33 \cdot 4 \%$, and $24 \cdot 8 \%$ ). For children diagnosed during 2010-14, 5-year survival for acute lymphoblastic leukaemia ranged from $\mathbf{4 9 . 8 \%}$ in Ecuador to $\mathbf{9 5 . 2 \%}$ in Finland. 5-year survival from brain tumours in children is higher than for adults but the global range is very wide (from $28.9 \%$ in Brazil to nearly $80 \%$ in Sweden and Denmark).

Interpretation The CONCORD programme enables timely comparisons of the overall effectiveness of health systems in providing care for 18 cancers that collectively represent $75 \%$ of all cancers diagnosed worldwide every year. It contributes to the evidence base for global policy on cancer control. Since 2017, the Organisation for Economic Co-operation and Development has used findings from the CONCORD programme as the official benchmark of cancer survival, among their indicators of the quality of health care in 48 countries worldwide. Governments must recognise population-based cancer registries as key policy tools that can be used to evaluate both the impact of cancer prevention strategies and the effectiveness of health systems for all patients diagnosed with cancer.

Funding American Cancer Society; Centers for Disease Control and Prevention; Swiss Re; Swiss Cancer Research foundation; Swiss Cancer League; Institut National du Cancer; La Ligue Contre le Cancer; Rossy Family Foundation; US National Cancer Institute; and the Susan G Komen Foundation.

\section{Introduction}

The incidence of cancer continues to rise, both in highincome countries and, especially, in low-income and middle-income countries. Prevention is crucial, but implementation has been slow and incomplete, even in high-income countries. Prevention is a long-term strategy, and not all cancers can be prevented. ${ }^{1}$ To reduce cancer mortality, reduction of cancer incidence and improvement of cancer survival are both necessary.

Many patients will continue to be diagnosed with cancer every year for decades to come: an estimated 14 million patients a year worldwide around $2012,{ }^{2}$ with a
Published Online January 30, 2018 http://dx.doi.org/10.1016/ S0140-6736(17)33326-3

See Online/Comment http://dx.doi.org/10.1016 S0140-6736(18)30155-7

*Members are listed at the end of the Article

Cancer Survival Group Department of Non-Communicable Disease Epidemiology, London School of Hygiene \& Tropical Medicine, London, UK (C Allemani PhD, V Di Carlo MSc, R Harewood MSc, M Matz PhD, M Nikšić PhD, A Bonaventure MD, Prof M P (oleman BM BCh); Population-based Cancer Registry Section, Division of Surveillance, Center for Cance Control and Information Services, National Cancer Center, Tokyo, Japan (T Matsuda PhD); Department of Radiology, Radiotherapy and Oncology, Northern State Medical University, Arkhangelsk, Russia (Prof M Valkov MD); Cancer Data Registry of Idaho, Boise, ID, USA (C) Johnson MPH). Department of Biostatistics, Université Claude Bernard, Lyon, France (ProfJ Estève PhD); Ibadan Cancer Registry, University City College Hospital, Ibadan, Dyo State, Nigeria

(Prof OJ Ogunbiyi MBBS); Department of Epidemiology, Universidade do Estado do Rio de Janeiro, Maracanã, Rio de Janeiro, Brazil (Prof G Azevedo e Silva PhD) National Office for Cancer Prevention and Control and National Central Cancer Registry, National Cancer Center, Beijing, China (W-Q Chen PhD); Department of Public Health, Balıkesir University, Balıkesir, Turkey 
(S Eser PhD); Department of Documentation and Quality, Danish Cancer Society, Copenhagen, Denmark (G Engholm MSc); National Cancer Registration and Analysis Service, Public Health England, London, UK (C A Stiller MSc); Registre des hémopathies malignes de la Gironde, Institut Bergonié, Bordeaux, France

(A Monnereau MD); French Network of Cancer Registries, Toulouse, France (A Monnereau); British Columbia Cancer Registry, BC Cancer Agency, Vancouver, BC, Canada (R Woods MSC);

Netherlands Cancer Registry Netherlands Comprehensive Cancer Organisation (IKNL), Utrecht, Netherlands (O Visser PhD); National Registry of Diseases Office, Health Promotion Board, Singapore (G H Lim MSc); Cancer Council Queensland, Fortitude Valley, QLD, Australia (Prof) Aitken PhD); and Division

of Cancer Prevention and

Control, Centers for Disease Control and Prevention,

Atlanta, GA, USA (H K Weir PhD)

Correspondence to:

Dr Claudia Allemani, Cancer

Survival Group, Department of

Non-Communicable Disease

Epidemiology, London School of

Hygiene \& Tropical Medicine, London WC1E 7HT, UK

claudia.allemani@Ishtm.ac.uk
Research in context

\section{Evidence before this study}

In 2015, the second cycle of the CONCORD programme

(CONCORD-2) established global surveillance of cancer survival as one of the key metrics of the effectiveness of health systems and to inform global policy on cancer control. This was done by analysis of individual records for 25.7 million patients diagnosed with one of ten common cancers during 1995-2009 and followed up to Dec 31, 2009. The data were provided by 279 population-based cancer registries in 67 countries.

CONCORD-2 revealed wide differences in cancer survival trends that were attributed to differences in access to early diagnosis and optimal treatment.

\section{Added value of this study}

CONCORD-3 covers almost 1 billion people worldwide. It includes 15 common cancers in adults and three common cancers in children. Data quality has improved. The results are timely, published within 3 years of the end of follow-up.

CONCORD-3 updates the worldwide surveillance of cancer survival to 2014. It includes data for over 37.5 million patients diagnosed with cancer during the 15-year period 2000-14. Data were provided by more than 320 population-based cancer registries in 71 countries and territories, including 27 countries of low or middle income; 47 countries provided data with $100 \%$ population coverage. The study now includes 18 cancers or groups of cancers: oesophagus, stomach, colon, rectum, liver, pancreas, lung, breast (women), cervix, ovary, prostate, and melanoma of the skin in adults, together with brain tumours, leukaemias, and lymphomas in both adults and children. These cancers represent $75 \%$ of all cancers diagnosed worldwide every year, in both low-income and high-income countries. The use of a similar study design and the same statistical methods as in CONCORD-2 enables the evaluation of survival trends for ten cancers over the 20-year period 1995-2014.
For the first time, worldwide trends in survival are also available for cancers of the oesophagus, pancreas, and brain, and lymphomas and leukaemias.

\section{Implications of all the available evidence}

The CONCORD programme enables comparative evaluation of the effectiveness of health systems in providing cancer care. It also contributes to the evidence base for global policy on cancer control. CONCORD monitors progress towards the overarching goal of the 2013 World Cancer Declaration, to achieve "major reductions in premature deaths from cancer, and improvements in quality of life and cancer survival" by 2020. It provides evidence to support WHO policy following the Cancer Resolution passed by the World Health Assembly in 2017. The International Atomic Energy Agency's Programme for Action on Cancer Therapy used CONCORD- 2 results in 2015 to launch its worldwide campaign to highlight the global divide in cancer survival, and to raise awareness of persistent inequalities in access to life-saving cancer services. The results were used in a Lancet Series on women's cancers in 2016. The US Centers for Disease Control and Prevention used the results in a 2017 supplement to the journal Cancer to inform cancer control policy designed to reduce racial differences in cancer survival.

CONCORD-3 can be expected to affect cancer control policy worldwide, especially in countries with low survival.

The Organisation for Economic Co-operation and Development published a subset of CONCORD-3 results in 2017 as the official benchmark of cancer survival, among their indicators of the quality of health care in 48 countries worldwide. The survival estimates will also form part of the Lancet Oncology Commission on childhood cancer in 2018. Future research will include examination of the impact on international differences in cancer survival of stage at diagnosis, compliance with treatment guidelines, and the quality of health care.
$50 \%$ projected increase to 21.6 million patients a year by $2030 .{ }^{3}$ Those patients will all need prompt diagnosis and optimal treatment to improve their survival. Monitoring the effectiveness of national and regional health systems in treating and caring for these patients becomes ever more crucial.

In 2016, the WHO Executive Board recommended strengthening health systems to ensure early diagnosis and accessible, affordable, high-quality care for all patients with cancer. ${ }^{3}$ The World Health Assembly followed up with a resolution on cancer control in May, 2017. It included recommendations that national cancer control strategies should aim to reduce late presentation and ensure appropriate treatment and care for potentially curable malignancies such as acute leukaemia in children "to increase survival, reduce mortality and improve quality of life". ${ }^{4}$

President Tabaré Vázquez of Uruguay and WHO Director-General Tedros Ghebreyesus have called for all countries "to provide universal health coverage, thereby ensuring all people can access needed preventive and curative health-care services, without falling into poverty". ${ }^{5}$ Their call relates to all non-communicable diseases, including cancer. Population-based cancer survival is one metric that can help evaluate whether all people have access to effective treatment services.

In 2015, the second cycle of the CONCORD programme (CONCORD-2) established global surveillance of cancer survival for the first time, ${ }^{6}$ with publication of trends in survival over the 15-year period 1995-2009 among patients diagnosed with cancer in 67 countries, home to two thirds ( 4.8 billion) of the world's population. In 40 countries, the data had $100 \%$ national population coverage. CONCORD-2 incorporated centralised quality control and analysis of individual data for 25676887 patients diagnosed with one of the ten common cancers that represented $63 \%$ of the global cancer burden in 2009. The 279 population-based 
registries covered a combined total population of 896 million people.

The US National Cancer Institute, in an invited commentary ${ }^{7}$ for The Lancet, noted that the global analyses of cancer survival in CONCORD-2 provided insights from countries with successful cancer control initiatives that could be applied in other regions, and that the availability of better data "provides a clearer picture of the effect of cancer control programmes on the ultimate goal of improving survival and reducing the effect of cancer on the social and economic development of countries".

The US Centers for Disease Control and Prevention described CONCORD-2 as the start of global surveillance of cancer survival, ${ }^{8}$ with survival estimates "that can be compared so scientists can begin to determine why survival differs among countries. This could lead to improvements in cancer control programs." The results from CONCORD-2 influenced national cancer control strategy in the UK in July, 2015. ${ }^{9,10}$ In September, 2015, the International Atomic Energy Agency's Programme for Action on Cancer Therapy used the results to launch a worldwide campaign ${ }^{11}$ to highlight the global divide in cancer survival, and to raise awareness of persistent inequalities in access to life-saving cancer services. ${ }^{12}$ Further analyses of survival trends and disparities by race and stage at diagnosis in 37 US states have been included in a supplement to $\mathrm{Cancer}^{13,14}$ designed to improve cancer control in the USA.

CONCORD-3 updates worldwide surveillance of cancer survival trends to include patients diagnosed up to 2014, with follow-up to Dec 31, 2014. In countries that were already involved, more registries are participating, and eight more countries have joined the programme. Follow-up for patients diagnosed during 2000-09 with one of the ten cancers included in CONCORD-2 has been updated. CONCORD-3 includes data for patients diagnosed during 2000-14 with one of 18 malignancies that represent $75 \%$ of the global cancer burden (table 1). In addition to information on stage at diagnosis, we have collected data on tumour grade and the first course of treatment. Findings are published within 3 years of the end of follow-up.

\section{Methods \\ Cancer registries}

We contacted 412 cancer registries in 85 countries: 20 in Africa (13 countries), 45 in Central and South America (15 countries), 68 in North America (two countries), 80 in Asia (20 countries), 189 in Europe (33 countries), and ten in Oceania (two countries).

When the data call for CONCORD-3 was issued in May, 2016, 12 of the 279 cancer registries that had participated in CONCORD-2 were no longer operational. The registry in Benghazi (Libya) had been disrupted by war, the registry in Macerata (Italy) had ceased operating, the Department of Health had ceased funding the UK National Registry of Childhood Tumours in 2013, and the

\begin{tabular}{|lccc}
\hline & $\begin{array}{l}\text { Overall } \\
(\mathbf{n}=\mathbf{1 4 0 6 7 8 9 4 )}\end{array}$ & $\begin{array}{l}\text { More developed regions } \\
(\mathbf{n}=6 \text { 053621) }\end{array}$ & $\begin{array}{l}\text { Less developed regions } \\
(\mathbf{n = 8} \text { 014 273) }\end{array}$ \\
\hline Oesophagus & $455784(3 \cdot 2 \%)$ & $86144(1 \cdot 4 \%)$ & $369640(4 \cdot 6 \%)$ \\
\hline Stomach & $951594(6 \cdot 8 \%)$ & $274509(4 \cdot 5 \%)$ & $677085(8 \cdot 4 \%)$ \\
\hline Colorectum & $1360602(9 \cdot 7 \%)$ & $736867(12 \cdot 2 \%)$ & $623735(7 \cdot 8 \%)$ \\
\hline Liver & $782451(5 \cdot 6 \%)$ & $134302(2 \cdot 2 \%)$ & $648149(8 \cdot 1 \%)$ \\
\hline Pancreas & $337872(2 \cdot 4 \%)$ & $187465(3 \cdot 1 \%)$ & $150407(1 \cdot 9 \%)$ \\
\hline Lung & $1824701(13 \cdot 0 \%)$ & $758214(12 \cdot 5 \%)$ & $1066487(13 \cdot 3 \%)$ \\
\hline Melanoma & $232130(1 \cdot 7 \%)$ & $191066(3 \cdot 2 \%)$ & $41064(0 \cdot 5 \%)$ \\
\hline Breast (women) & $1671149(11 \cdot 9 \%)$ & $788200(13 \cdot 0 \%)$ & $882949(11 \cdot 0 \%)$ \\
\hline Cervix & $527624(3 \cdot 8 \%)$ & $83078(1 \cdot 4 \%)$ & $444546(5 \cdot 5 \%)$ \\
\hline Ovary & $238719(1 \cdot 7 \%)$ & $99752(1 \cdot 6 \%)$ & $138967(1 \cdot 7 \%)$ \\
\hline Prostate & $1094916(7 \cdot 8 \%)$ & $741966(12 \cdot 3 \%)$ & $352950(4 \cdot 4 \%)$ \\
\hline $\begin{array}{l}\text { Brain and central } \\
\text { nervous system }\end{array}$ & $256213(1 \cdot 8 \%)$ & $88967(1 \cdot 5 \%)$ & $167246(2 \cdot 1 \%)$ \\
\hline Lymphomas & $451691(3 \cdot 2 \%)$ & $219255(3 \cdot 6 \%)$ & $232436(2 \cdot 9 \%)$ \\
\hline Leukaemias & $351965(2 \cdot 5 \%)$ & $141274(2 \cdot 3 \%)$ & $210691(2 \cdot 6 \%)$ \\
\hline All index cancers* & $10537411(74 \cdot 9 \%)$ & $4531059(74 \cdot 8 \%)$ & $6006352(74 \cdot 9 \%)$ \\
\hline
\end{tabular}

Data are from Globocan, 2012. ${ }^{15}$ Index cancer refers to a cancer or group of malignancies included in CONCORD-3. More developed regions refers to North America, Europe, Australia, New Zealand, and Japan; all other countries and regions are classified as less developed. ${ }^{15}$ These are UN designations intended for statistical convenience and do not reflect a judgment about the stage reached by a particular country or area in the development process. ${ }^{16}$ *Excluding non-melanoma skin cancer.

Table 1: Estimated number of patients diagnosed with an index cancer worldwide each year around 2012

nine English regional cancer registries had been replaced by a single cancer registry for England in 2013. Of the 267 remaining registries, nine could no longer provide up-to-date follow-up of all registered patients, whereas 13 did not reply to repeated approaches. Data from the Tirol (Austria) registry are no longer reported separately from the Austrian national estimates. In all, 244 (87\%) of the 279 registries ( 63 of the 67 countries) that participated in CONCORD-2 submitted data (appendix p 266).

Of the 133 registries that had not previously participated in the CONCORD programme, 108 agreed to do so. Of these, 85 (78\%) registries in 12 countries submitted data, whereas 11 were unable to complete follow-up of registered patients with cancer for their vital status, nine made no further contact, and three signed up too late (appendix p 266)

Of the 329 registries that submitted data, seven were excluded because their data were not compliant with the protocol and could not be rectified in time. These exclusions affected the only participating registry or registries from several countries: Tunisia (Central Region), Bosnia and Herzegovina (Republika Srpska), Saudi Arabia, and Serbia (Central Region and Vojvodina). We analysed data provided by 322 cancer registries ( $81 \%$ of the 400 operational registries invited) in 71 countries and territories (appendix p 266), for patients diagnosed with cancer during the 15-year period 2000-14, with data on their vital status at least 5 years after diagnosis, or at Dec 31, 2014.

Eight countries from four world regions are participating in the global surveillance of cancer survival 
for the first time: Morocco (Africa); Costa Rica (national), Mexico (children, national), and Peru (Central and South America); Iran, Kuwait (national), and Singapore (national; Asia), and Greece (children, national; Europe).

\section{Ethical approvals}

We maintain approvals from the Confidentiality Advisory Group of the UK's statutory Health Research Authority (HRA; reference ECC 3-04(i)/2011; last update March 3, 2017), the National Health Service Research Ethics Service (11/LO/0331; Feb 21, 2017), and the London School of Hygiene \& Tropical Medicine (12171; Sept 6, 2017). The HRA also approves the Cancer Survival Group's System-Level Security Policy, governing data security. One investigator (MPC) maintains triennial certification with the Collaborative Institutional Training Initiative in Human Subjects Research for Biomedical Investigators (CITI Program; ID3327653; certification updated May 2, 2016). We maintain statutory or ethical approvals and data sharing agreements, usually with annual renewal, in 85 other jurisdictions participating in the CONCORD programme. Registries in all other jurisdictions obtain local approval. The data belong to the participating registries and are only used for purposes agreed in the CONCORD protocol.

Participants transmit data via a specially configured file transmission utility with 256-bit Advanced Encryption Security. The utility automatically generates a random, strong, one-time password for each data file at the time of transmission, and emails it to a different address. Neither the password nor the address are seen by the sender. This avoids the need for confirmation of passwords by email or telephone. Tumour records are effectively anonymised: they do not contain the patient's name, address, postcode, or any national identity or social security number. All variables are numeric or alphanumeric codes. Each registry is sent a set of unique codes that must be used in naming each cancer data file, including distinct filenames for any retransmission. The codes have no meaning outside of the study. Data files thus contain no information that could be used to identify a person or a cancer registry, and neither the name nor the content of the file would indicate that the file contains cancer data. This enhances security and facilitates efficient handling of thousands of data files.

\section{Protocol}

The CONCORD-3 protocol defining the data structure, file transmission procedures, and statistical analyses was expanded and updated from the CONCORD-2 protocol, with the inclusion of variables on five additional cancers or groups of malignancies, tumour grade, and the modality and date of the first course of treatment by surgery, radiotherapy, or systemic therapy.

In a study of this scale, adherence to protocol is crucial. The protocol and analytic approaches were discussed with CONCORD Working Group members from 27 countries at a 1-day meeting in Marrakesh, Morocco, on Oct 17, 2016. The protocol was also discussed at workshops in China, Romania, Russia, Singapore, and the USA (for North America), and in conference calls with Costa Rica, Hong Kong, Malaysia, Mauritius, Mexico, and Mongolia.

English is still a barrier to communication in many countries, so the CONCORD-3 protocol was translated into eight other languages: Arabic, Chinese (Mandarin), French, Italian, Japanese, Portuguese, Russian, and Spanish. Translations were done by native speakers in the CONCORD Central Analytic Team in London or the wider CONCORD Working Group, and checked against the English original by other native speakers. The protocol was made available to participants in all nine languages on the CONCORD website. The Central Analytic Team communicates with participants in six languages.

We examined survival for 18 cancers or groups of malignancies (referred to as index cancers): oesophagus, stomach, colon, rectum, liver, pancreas, lung, melanoma of the skin, breast (women), cervix, ovary, and prostate in adults (15-99 years); brain tumours, myeloid, and lymphoid malignancies in adults; and brain tumours, acute lymphoblastic leukaemia, and lymphomas in children ( $0-14$ years). Collectively, these cancers accounted for about $75 \%$ of the estimated number of patients diagnosed with cancer worldwide each year around 2012 (table 1). The overall proportion is very similar in North America, Europe, Australia, New Zealand, and Japan (referred to as developed countries ${ }^{15}$ ) and in other world regions (referred to as developing countries ${ }^{15}$ ), but it varies widely between cancers: prostate cancer is proportionately three times more common in developed countries, and cervical cancer is four times more common in developing countries (table 1).

Solid tumours were defined by anatomical site (topography), and the leukaemias, lymphomas, and melanoma of the skin by morphology (table 2). Topography and morphology were coded to the International Classification of Diseases for Oncology (third edition, ICD-O-3), ${ }^{17}$ including its first revision. ${ }^{18}$ We restricted estimation of survival for melanomas to those arising in the skin, including the skin of the labia majora, vulva, penis, and scrotum (table 2). Melanomas arising in internal organs were included with all other malignancies in those organs. For ovarian cancer, we included the fallopian tube, uterine ligaments, and adnexa, as well as the peritoneum and retroperitoneum, where high-grade serous ovarian carcinomas are often detected. ${ }^{21}$ Registries were not asked to select cancers by sex, although some did so. Where datasets did include records for breast cancer in men, the proportion was consistently around $0.7 \%$; these records were excluded. We also excluded small numbers of retroperitoneal malignancies in men, as well as Kaposi's sarcoma, and tumours in solid organs with haemopoietic morphology.

Registries provided data for all haemopoietic malignancies (ICD-O-3 morphology codes in the 


\begin{tabular}{|c|c|c|c|c|c|c|c|c|c|c|}
\hline & \multirow[t]{3}{*}{ Topography or morphology codes* } & \multirow[t]{3}{*}{ Description } & \multicolumn{8}{|c|}{ Contributing countries and registries } \\
\hline & & & \multicolumn{2}{|l|}{$2000-04$} & \multicolumn{2}{|l|}{ 2005-09 } & \multicolumn{2}{|l|}{$2010-14$} & \multicolumn{2}{|c|}{ Any period (2000-14) } \\
\hline & & & Countries & Registries & Countries & Registries & Countries & Registries & Countries & Registries \\
\hline Oesophagus & C15.0-C15.5, C15.8-C15.9 & Oesophagus & 55 & 249 & 59 & 287 & 58 & 273 & 60 & 290 \\
\hline Stomach & C16.0-C16.6, C16.8-C16.9 & Stomach & 57 & 252 & 62 & 293 & 60 & 277 & 62 & 294 \\
\hline Colon & C18.0-C18.9, C19.9 & $\begin{array}{l}\text { Colon and rectosigmoid } \\
\text { junction }\end{array}$ & 57 & 251 & 64 & 294 & 64 & 280 & 65 & 296 \\
\hline Rectum & $\mathrm{C} 20.9, \mathrm{C} 21.0-\mathrm{C} 21.2, \mathrm{C} 21.8$ & $\begin{array}{l}\text { Rectum, anus, and anal } \\
\text { canal }\end{array}$ & 56 & 250 & 63 & 292 & 63 & 278 & 64 & 294 \\
\hline Liver & $\mathrm{C} 22.0-\mathrm{C} 22.1$ & $\begin{array}{l}\text { Liver and intrahepatic } \\
\text { bile ducts }\end{array}$ & 56 & 250 & 60 & 289 & 60 & 275 & 61 & 291 \\
\hline Pancreas & $\mathrm{C} 25.0-\mathrm{C} 25.4, \mathrm{C} 25.7-\mathrm{C} 25.9$ & Pancreas & 55 & 249 & 58 & 288 & 58 & 274 & 59 & 290 \\
\hline Lung & С $34.0-C 34.3$, C34.8-C34.9 & Lung and bronchus & 57 & 250 & 61 & 289 & 61 & 275 & 61 & 290 \\
\hline $\begin{array}{l}\text { Melanoma of } \\
\text { the skin }\end{array}$ & $\begin{array}{l}\text { 8720-8790 provided topography was } \\
\text { C44.0-C } 44.9, C 51.0, C 51.9, C 60.9 \text {, or } C 63.2\end{array}$ & $\begin{array}{l}\text { Melanoma of the skin, } \\
\text { including skin of labia } \\
\text { majora, vulva, penis, and } \\
\text { scrotum }\end{array}$ & 55 & 239 & 58 & 278 & 59 & 266 & 59 & 281 \\
\hline $\begin{array}{l}\text { Breast } \\
\text { (women) }\end{array}$ & C50.0-C50.6, C50.8-C50.9 & Breast & 59 & 255 & 64 & 295 & 65 & 282 & 66 & 298 \\
\hline Cervix & C53.0-C53.1, C53.8-C53.9 & Cervix uteri & 57 & 253 & 63 & 293 & 62 & 277 & 64 & 295 \\
\hline Ovary & C48.0-C48.2, C56.9, C57.0-C57.4, C57.7-C57.9 & $\begin{array}{l}\text { Ovary, fallopian tube and } \\
\text { uterine ligaments, other } \\
\text { and unspecified female } \\
\text { genital organs, } \\
\text { peritoneum, and } \\
\text { retroperitoneum }\end{array}$ & 56 & 249 & 61 & 288 & 59 & 272 & 61 & 289 \\
\hline Prostate & C61.9 & Prostate gland & 58 & 249 & 62 & 289 & 62 & 275 & 62 & 290 \\
\hline Brain (adults) & C71.0-C71.9 & Brain (adults) & 55 & 247 & 58 & 283 & 58 & 269 & 59 & 286 \\
\hline $\begin{array}{l}\text { Myeloid } \\
\text { (adults) }{ }^{\dagger}\end{array}$ & $\begin{array}{l}\text { 9740, 9741, 9742, 9800, 9801, 9805, 9806, } \\
\text { 9807, 9808, 9809, 9840, 9860, 9861, 9863, } \\
\text { 9865, 9866, 9867, 9869, 9870, 9871, 9872, } \\
\text { 9873, 9874, 9875, 9876, 9891, 9895, 9896, } \\
\text { 9897, 9898, 9910, 9911, 9920, 9930, 9931, } \\
\text { 9945, 9946, 9950, 9960, 9961, 9962, 9963, } \\
\text { 9964, 9975, 9980, 9982, 9983, 9984, 9985, } \\
\text { 9986, 9987, 9989, 9991, } 9992\end{array}$ & All myeloid malignancies & 56 & 249 & 59 & 280 & 60 & 268 & 61 & 286 \\
\hline $\begin{array}{l}\text { Lymphoid } \\
\text { (adults) } \dagger\end{array}$ & $\begin{array}{l}\text { 9590, 9591, 9596, 9597, 9650-9655, 9659, } \\
\text { 9661-9665, 9667, 9670, 9671, 9673, 9675, } \\
\text { 9678, 9679, 9680, 9684, 9687-9691, 9695, } \\
\text { 9698, 9699, 9700-9702, 9705, 9708, 9709, } \\
\text { 9712, 9714, 9716-9719, 9725-9729, 9731-9735, } \\
\text { 9737, 9738, 9760-9762, 9764, 9811-9818, 9820, } \\
\text { 9823, 9826, 9827,9831-9837, 9940, 9948 }\end{array}$ & $\begin{array}{l}\text { All lymphoid } \\
\text { malignancies }\end{array}$ & 57 & 250 & 60 & 284 & 61 & 271 & 62 & 289 \\
\hline $\begin{array}{l}\text { Brain } \\
\text { (children) }\end{array}$ & C71.0-C71.9 & Brain (children) & 54 & 219 & 58 & 257 & 60 & 245 & 60 & 260 \\
\hline $\begin{array}{l}\text { Acute } \\
\text { lymphoblastic } \\
\text { leukaemia } \\
\text { (children)‡ }\end{array}$ & $\begin{array}{l}\text { 9835-9837; plus } 9811-9818 \text { provided } \\
\text { topography was } C 42.0, \text { C } 42.1, \text { C } 42.3 \text {, C } 42.4 \text {, } \\
\text { or C } 80.9\end{array}$ & $\begin{array}{l}\text { Precursor-cell acute } \\
\text { lymphoblastic leukaemia }\end{array}$ & 56 & 214 & 60 & 247 & 61 & 233 & 61 & 254 \\
\hline $\begin{array}{l}\text { Lymphoma } \\
\text { (children) }\end{array}$ & $\begin{array}{l}\text { 9590, 9591, 9596, 9597, 9650-9655, 9659, } \\
9661-9665,9667,9670,9671,9673,9675, \\
\text { 9678-9680, 9684, 9687-9691, 9695, } \\
\text { 9698-9702, 9705, 9708, 9709, 9712, 9714, } \\
\text { 9716-9719, 9725-9729, 9731-9735, 9737, 9738, } \\
\text { 9740-9742, 9750-9762, 9764-9769, 9970, } \\
\text { 9971; plus 9811-9818 provided topography was } \\
\text { not C42.0, C42.1, C42.3, C42.4, or C80.9 }\end{array}$ & All lymphomas & 55 & 214 & 60 & 253 & 62 & 235 & 62 & 257 \\
\hline $\begin{array}{l}\text { Some registries co } \\
\text { at some point dur } \\
\text { were defined by } \mathrm{H} \\
\text { Cancer (third editi }\end{array}$ & $\begin{array}{l}\text { tributed data for selected cancers or calendar periods, so the } \\
\text { g } 2000-14 \text { is thus greater than or equal to the number in ar } \\
\text { IEMACARE }{ }^{9} \text { groups } 1-19 \text { and myeloid malignancies by HAE } \\
\text { nn }{ }^{20} \text { incorporating morphology codes from the first revision }\end{array}$ & $\begin{array}{l}\text { ne number of participating cour } \\
\text { any } 5 \text {-year period. *Internationa } \\
\text { EMACARE groups } 20-25 \text {, incorn } \\
\text { nof ICD- } 0-3^{18} \text { was used to defin }\end{array}$ & $\begin{array}{l}\text { ntries alsc } \\
\text { al Classific } \\
\text { porating } \\
\text { ne childhe }\end{array}$ & $\begin{array}{l}\text { ries by can } \\
\text { on of Disea } \\
\text { rphology c } \\
\text { acute lym }\end{array}$ & sticteu & $\begin{array}{l}\text { period. The } \\
\text { (ICD-O-3), } \\
\text { st revision } \\
\text { emia (group }\end{array}$ & $\begin{array}{l}\text { mber of } \\
\text { cluding it } \\
\text { CD-O-3. } \\
\text { 1) and lyn }\end{array}$ & $\begin{array}{l}\text { atries and } \\
\text { rst revision } \\
\text { Internatic } \\
\text { oma in chi }\end{array}$ & $\begin{array}{l}\text { ries that con } \\
\text { ymphoid ma } \\
\text { llassification } \\
\text { (group II). }\end{array}$ & $\begin{array}{l}\text { ntributed data } \\
\text { ralignancies } \\
\text { hof Childhood }\end{array}$ \\
\hline
\end{tabular}


range 9590-9992) in adults and children, to minimise differences in the spectrum of leukaemias and lymphomas submitted for analysis. In consultation with specialists in the HAEMACARE ${ }^{19}$ and InterLymph ${ }^{22,23}$ groups, we agreed to analyse survival for adults in two broad groups: lymphoid malignancies (HAEMACARE groups 1-19) and myeloid malignancies (groups 20-25; table 2; appendix pp 2-5).

For children, we agreed to present survival estimates separately for acute lymphoblastic leukaemia and lymphomas, based on ICD-O-3 codes, grouped according to the third edition of the International Classification of Childhood Cancer. ${ }^{20}$ The first revision of ICD-O-3, published in 2013, ${ }^{18}$ introduced eight new entities for acute lymphoblastic leukaemia or lymphoma (morphology codes 9811-9818). These new entities were not used at all by registries in 42 of the 58 countries that submitted data for children diagnosed with acute lymphoblastic leukaemia during 2010-14, and very rarely in eight countries (ie, the combined number of children coded to a new entity was fewer than 100), but the proportions ranged from $11 \%$ to $89 \%$ in large datasets from Australia, Belgium, Canada, the Netherlands, Puerto Rico, Singapore, Taiwan, and the USA. The overall proportion for all 58 countries combined during 2010-14 was $29 \%$ (10679 of 36867 children). We therefore included the new entities in all analyses. We included them among the acute lymphoblastic leukaemias if the anatomical site was coded as blood, bone marrow, reticulo-endothelial, or haemopoietic system not otherwise specified (C42.0-42.1, C42.3-42.4), or unknown primary site (C80.9). Otherwise, such malignancies were included with the lymphomas (appendix pp 2-5).

Survival analyses include only primary, invasive malignancies (ICD-O-3 behaviour code 3), except for the brain, where benign tumours (behaviour code 0 ) are also included. To facilitate quality control and comparison of the intensity of early diagnostic and screening activity, registries were asked to provide data for all registered malignancies at each index site, including those that were benign, of uncertain or borderline malignancy (behaviour code 1), in situ (behaviour code 2), metastatic (behaviour code 6), or uncertain whether primary or metastatic (behaviour code 9).

Registries were asked to provide full dates (day, month, and year) of birth, diagnosis, and death or last known vital status, both for quality control and to enable comparable estimation of survival. ${ }^{24}$ Where the day or month of birth, or the day of the date of diagnosis, or the day or month of the date of last known vital status was missing, we used an algorithm (details on request) to standardise the imputation of missing components of dates for all populations.

Participating registries completed a questionnaire on their methods of operation, including data definitions, data collection procedures, coding of anatomical site, morphology and behaviour, the tracing of patients registered with cancer to ascertain their vital status, and how tumour records are linked with data on vital status.

Patients diagnosed with two or more primary cancers at different index sites during 2000-14 were included in the analyses for each cancer-eg, colon cancer in 2005 followed by a breast cancer in 2010. Survival was measured from the date of diagnosis until death, loss to follow-up, or censoring. We retained the most complete record for patients with synchronous primary cancers in the same organ. If a patient was registered with two or more primary malignancies in the same index site during 2000-14 (metachronous primaries), only the first was included in analyses.

North American registries define multiple primary cancers under the rules of the Surveillance Epidemiology and End Results programme. ${ }^{25}$ Those rules accept more cancers as new primary cancers than do the rules of the International Association of Cancer Registries (IACR), ${ }^{26}$ which are used by most cancer registries in other continents. The North American Association of Central Cancer Registries (NAACCR) kindly updated the program developed for CONCORD-2 to enable all North American registries to recode their entire incidence databases to the IACR multiple primary rules before their datasets for 2000-14 were extracted for CONCORD-3.

Countries and territories were defined by their United Nations (UN) name, continent, and code as of $2015 .{ }^{16}$ The names of jurisdictions used in the text, tables, graphics, maps, and appendix are based on those used for statistical purposes by the Statistics Division of the UN Secretariat; similarly, we use the terms "national coverage" to contrast with "regional coverage" for statistical purposes. These designations and the presentation of data here do not imply any assumption regarding the political affiliation of countries or territories, or the expression of any opinion whatsoever on the part of the CONCORD programme concerning the legal status of any country, territory, city, or area, or of its authorities, or concerning the delimitation of its frontiers or boundaries. Some names have been shortened for convenience (eg, Korea for South Korea): this does not carry any political significance.

Cyprus is a Member State of the European Union, but it is part of Asia. Costa Rica, Cuba, Guadeloupe, Martinique, Mexico, and Puerto Rico (Caribbean and Central America) were grouped with South America as Central and South America. World maps and 29 regional maps were prepared in ArcGIS Release 10.3, ${ }^{27}$ using digital boundaries (shapefiles) from the database of global administrative areas (GADM 2.8).

The population coverage of the data from participating registries is given as the proportion of the country or jurisdiction's population, taken from the UN Population Division database for $2014,{ }^{28}$ or from the authorities for Australia, Guadeloupe, Hong Kong, Poland, Portugal, and Taiwan, or the registries concerned. Belarus, Greece, and Mexico provided data only for childhood cancers, so the populations used were for children (0-14 years), 
and Mali, Mongolia, and Morocco only provided cancer data for women, so we used the female populations.

\section{Quality control}

As for the previous cycle of the CONCORD programme, we carried out data quality checks in three phases: protocol adherence, exclusions, and editorial checks. After each phase, a detailed report was sent to each cancer registry for discussion and correction of data where required.

First, we sent registries a report showing the percentage compliance with the protocol for each of 51 variables in each cancer file. Compliance of less than $100 \%$ required correction or resubmission of data. Next, we checked for logical inconsistencies between the variables in each tumour record. Exclusion criteria were defined a priori, on the basis of experience from CONCORD-2, and extended to cover features of some of the five additional cancers such as Ann Arbor stage for the lymphomas and 14 additional variables on tumour grade and treatment. The variables in each record were checked for logical coherence against 20 sets of criteria, including eligibility (eg, age and tumour behaviour), definite errors (eg, sexsite errors, invalid dates, impossible date sequence, and missing vital status), and possible errors, including a wide range of inconsistencies between age, tumour site, and morphology. ${ }^{6}{ }^{62}$ Registries were sent exclusion reports for each index cancer and each calendar period, summarising the number of tumour records with each type of definite or possible error, the number registered from a death certificate only (DCO) or detected at autopsy, and the number and proportion of eligible patients whose data could be included in survival analyses. Registries were invited to request details of tumour records in which errors had been detected. Many registries used this information to update their databases. Where errors in classification, coding, or pathological assignment were identified, registries were asked to correct and resubmit their data.

Finally, we examined the proportion of tumour records with morphological verification of the diagnosis, whether from histology of a biopsy or surgical specimen, cytology of a smear or bone marrow aspirate, or from imaging or biomarkers, including tumours with a specific morphology code. We also examined the proportion of cases with non-specific morphology; the distributions of the day and month of the dates of birth, diagnosis, and last known vital status; and the proportion of patients who died within 30 days, were lost to follow-up, or were censored within 5 years of diagnosis.

\section{Follow-up for vital status}

Cancer registries use various methods to determine the vital status (alive, dead, emigrated, or lost to follow-up) of patients registered with cancer. ${ }^{6}$ Among 243 registries that provided specific information on follow-up procedures, 242 (99\%) determine the vital status of registered patients with cancer using passive follow-up techniques in which tumour registration records are regularly linked to a regional or national index of all death registrations, regardless of the cause of death. Linkages are usually based on a national identity or social security number that is stored in both records. Such linkages are increasingly done electronically, but manual scrutiny of printed lists is still required in places. Tumour records that match to a death record are updated with the date of death. Some registries routinely receive paper or electronic death certificates for their territory but this is insufficient on its own because death certificates that do not mention cancer are rarely included. Transcription errors can arise with identity numbers, so variables such as the name, sex, and date of birth are often used to improve the probability of an accurate match between a cancer record and a death registration.

Many registries use electoral registers, hospital records, or official databases, such as social insurance, health insurance, and driving licences, to determine the date on which a patient was last known or believed to have been alive. Patients recorded as having migrated beyond the registry's jurisdiction, or to another country, might be recorded as lost to follow-up because the patient's eventual death is unlikely to be recorded: they are censored from survival analysis on that date.

Active follow-up techniques are also used by 124 (51\%) of the 243 registries, which routinely contact the treating physician, general practitioner, or hospital administration to determine the vital status for each registered patient, often on a quarterly or annual basis. Some registries also determine the vital status by contact with the patient's family, by telephone or home visit, or with the village administration.

Registries were asked to submit data with follow-up for at least 5 years or, for patients diagnosed during 2010-14, until Dec 31, 2014. Registration and follow-up for patients diagnosed in 2000-09 was updated and new datasets were submitted.

Patients registered solely from a death certificate or diagnosed at autopsy were excluded from analyses because their survival time is unknown.

\section{Statistical analysis}

Most registries submitted data for patients diagnosed between 2000 and 2014, with follow-up to 2014, although some registries only began operation after 2000 or provided data for less than 15 years. The study design we used to examine survival trends among patients diagnosed in three consecutive 5-year calendar periods was "cohort, cohort, period". We used the cohort approach to estimate survival for patients diagnosed during 2000-04 and 2005-09 and the period approach for patients diagnosed during 2010-14. This design was also used for CONCORD-2, ${ }^{6}$ so it enables us to examine global trends in survival over a 20 -year period by 
including the estimates for patients diagnosed during 1995-99.

The cohort approach is considered the gold standard ${ }^{30,31}$ because it provides a survival estimate for a group of patients who were diagnosed during the same year or period, are likely to have been treated in similar fashion, and who have all been followed up for at least the duration of survival required, such as 5 years. This approach to the estimation of survival is easy to interpret, but other approaches are required when some patients have been followed up for less than 5 years.

We used the cohort approach for patients diagnosed in 2000-04 and 2005-09 because in most datasets all patients had been followed up for at least 5 years. We used the period approach ${ }^{32}$ for patients diagnosed during 2010-14 because 5 years of follow-up data were not available for all patients. This combination of cohort and period approaches facilitates monitoring of cancer survival trends over an extended time span, from the earliest to the most recent years of cancer registration for which follow-up data are available (appendix p 267). ${ }^{33}$

To ensure comparability of survival trends from 1995, we estimated net survival up to 5 years after diagnosis for both adults and children. Net survival is the cumulative probability of surviving up to a given time since diagnosis (eg, 5 years) after correcting for other causes of death (background mortality). We used the Pohar Perme estimator, ${ }^{34}$ which takes unbiased account of the higher competing risks of death in elderly people, implemented with the algorithm stns ${ }^{35}$ in Stata (version 14)

To control for the wide differences in background mortality between participating jurisdictions and over time, we produced 6210 life tables of all-cause mortality rates for each calendar year during 2000-14 in the general population of each country or registry territory, by single year of age, by sex, and by race or ethnicity in Australia (Northern Territory: Indigenous or non-Indigenous), Israel (Arab or Jewish), New Zealand (Māori or nonMāori), and Singapore (Chinese, Malay, or Indian). For 127 registries, we obtained complete life tables that did not require interpolation or smoothing for each calendar year in 2000-14.

For 193 registries, the method of life table construction depended on whether we received raw data (numbers of deaths and populations) or mortality rates, and on whether the raw data or the mortality rates were by single year of age (ie, complete) or by 5 -year age group (ie, abridged).

For 108 registries, we obtained death and population counts from the registry or the relevant national statistical authority. We derived life tables for 2001 and 2013 if possible, each centred on 3 calendar years of data (eg, 2000-02 or 2012-14) to increase the robustness of the rates. We constructed raw mortality rates from the death and population counts using a Poisson regression model with flexible functions, ${ }^{36}$ then smoothed and extended the rates to obtain complete life tables by sex and single year of age up to age 99 years. Life tables for each calendar year in 2002-12 were created by linear interpolation between the 2001 and 2010 life tables. ${ }^{37}$ Rather than extrapolate, we used the life table centred on 2001 for 2000, and the life table centred on 2013 for 2014.

For 56 registries that provided abridged mortality rates, or complete mortality rates that were not smoothed, we used the Ewbank relational model ${ }^{38}$ with three or four parameters to interpolate (if abridged) and smooth the mortality rates for the registry territory against a highquality smooth life table for a country with a similar pattern of mortality by age. ${ }^{39}$

Each set of life tables was checked with a standardised statistical summary on the earliest and latest year of available data, showing the data source and the method of construction and smoothing. For each sex and, where relevant, each race or ethnicity, the reports show the life expectancy at birth, the probability of death in the age bands 15-59, 60-84, and 85-99 years, and semi-log plots of the age-mortality rates from 0 to 99 years, showing both the raw datapoints and the final smoothed life-table curve, and the model residuals by age group (appendix pp 268-271).

Collection of authoritative raw data on the numbers of deaths and populations by age, sex, and calendar year or period in participating jurisdictions proved more difficult than in 2013-14. For 29 registries, no reliable data on allcause mortality could be obtained for the registry territory. We took national life tables published by the UN Population Division ${ }^{28}$ and interpolated and extended them to age 99 years with the Elandt-Johnson method..$^{40}$

For the 42 participating states in the USA, we used life tables by state, race, and socioeconomic status, provided by the US National Cancer Institute (Mariotto A; personal communication on Jan 26, 2016).

For each country, registry, and calendar period, we present age-standardised net survival estimates for each cancer at 5 years after diagnosis. For adults, we used the International Cancer Survival Standard (ICSS) weights, ${ }^{4}$ in which age at diagnosis is categorised into five groups: 15-44, 45-54, 55-64, 65-74, and 75-99 years and, for prostate cancer, $15-54,55-64,65-74,75-84$, and 85-99 years. Of the three sets of ICSS weights, we used group 2 (cancers for which incidence does not increase steeply with age) for melanoma of the skin, cervix uteri, and brain (adults), and group 1 (cancers for which incidence does increase steeply with age) for oesophagus, stomach, colon, rectum, liver, pancreas, lung, breast, ovary, and prostate, and both groups of haemopoietic malignancies. For children, we estimated survival for the age groups $0-4,5-9$, and 10-14 years; we obtained age-standardised estimates by assigning equal weights to the three age-specific estimates. ${ }^{41,42}$

Cumulative survival probabilities in the range $0-1$ are presented for convenience as percentages in the range $0-100 \%$. 95\% CIs for both unstandardised and 


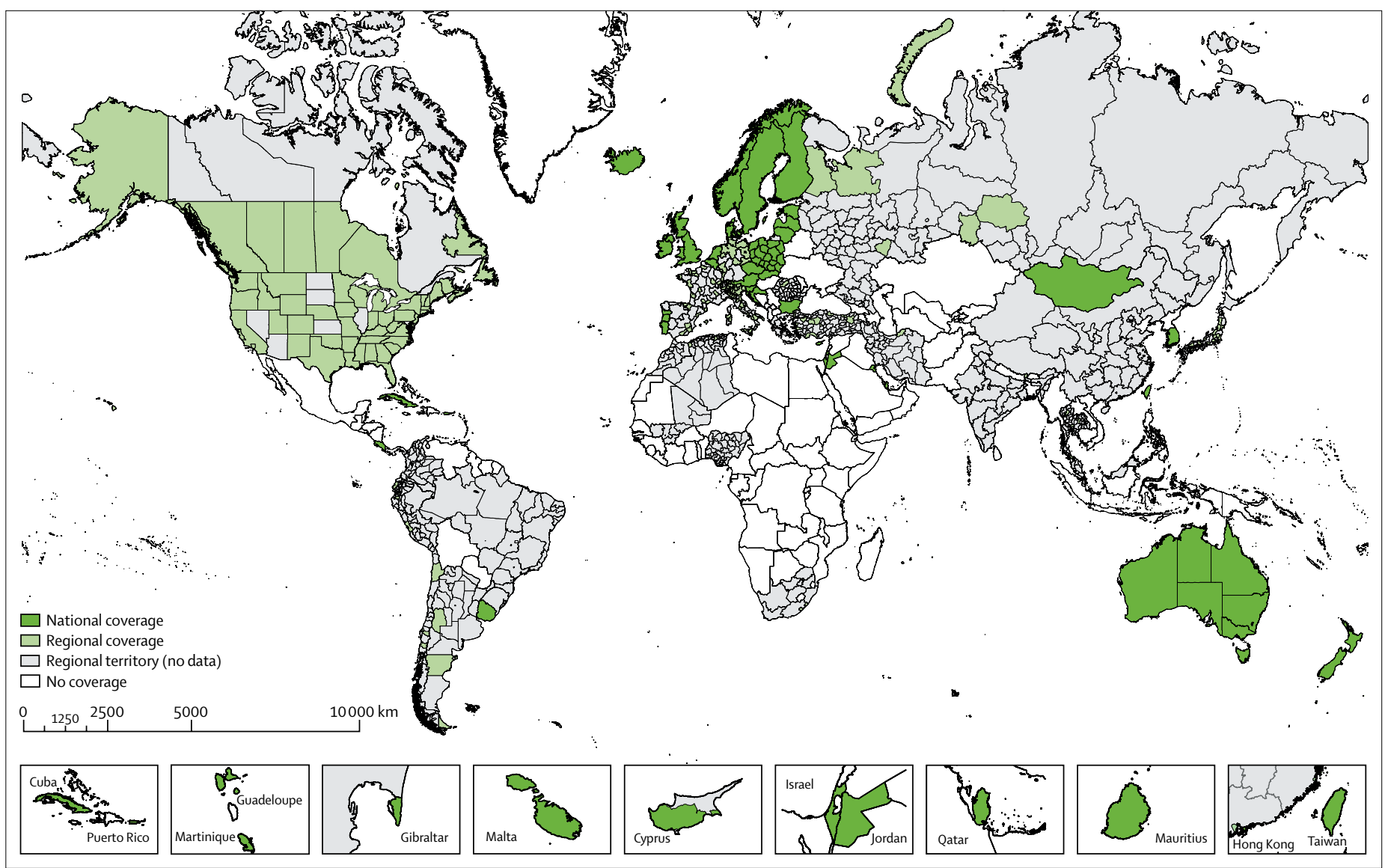

Figure 1: Participating countries and regions: world (adults)

Registries in smaller countries are shown in boxes, at different scales. See appendix (pp 178-208) for regional maps and for world map for childhood cancers.

age-standardised survival estimates were derived assuming a normal distribution, truncated to the range $0-100$. Standard errors to construct the CIs were derived with the Greenwood method. ${ }^{43}$ If no death or censoring occurred within 5 years, or if all patients died within 5 years (survival probability 1 or 0 ), we obtained a binomial approximation for the lower or upper bound, respectively, of the CI. ${ }^{30}$

We did not estimate survival if fewer than ten patients were available for analysis. If 10-49 patients were available for analysis in a given calendar period, we only estimated survival for all ages combined. If 50 or more patients were available, we attempted survival estimation for each age group. If a single age-specific estimate could not be obtained, we merged the data for adjacent age groups and assigned the combined estimate to both age groups before standardisation for age. If two or more age-specific estimates could not be obtained, we present only the unstandardised estimate for all ages combined. We did not merge data between consecutive calendar periods.

We considered survival estimates as less reliable if $15 \%$ or more of patients were lost to follow-up or censored alive within 5 years of diagnosis. For patients diagnosed in 2010 or later, this criterion was applied for patients censored alive before Dec 31, 2014, the study closure date. Estimates are also considered less reliable if $15 \%$ or more of patients were registered only from a death certificate or at autopsy and excluded from analysis, because their survival is unknown. Finally, estimates are also considered less reliable if $15 \%$ or more of patients were excluded from analysis because one or more dates was incomplete: unknown year of birth, unknown month or year of diagnosis, or unknown year of last known vital status.

The pooled estimates for countries with more than one registry do not include data from registries for which the estimates were less reliable. Less reliable estimates are shown with a flag in figures and tables when they are the only available information from a given country or territory.

\section{Role of the funding source}

The funding sources played no part in the design, data collection, quality control, analysis, interpretation of the findings, writing of the manuscript, or the decision to submit for publication. The corresponding author had full access to all data and responsibility for submission for publication. 


\section{Results}

\section{The CONCORD database 2000-14}

We analysed data for 322 cancer registries in 71 countries in Africa (eight registries, six countries), Central and South America (33 registries, 13 countries), North America (57 registries, two countries), Asia (66 registries, 17 countries), Europe (149 registries, 31 countries), and Oceania (nine registries, two countries; figure 1).

For 47 countries, data were provided with $100 \%$ coverage of the national population: 41 countries for both adults and children, and six for children only (Argentina, Belarus, France, Greece, Mexico, and Switzerland; table 3). In the other countries, population coverage varied from less than $1 \%$ in India to $86 \%$ in the USA (tables 4,5 ). 80 cancer registries joined the CONCORD programme for the first time. The 322 participating registries covered a combined population of almost 1 billion people around 2014 (989082 244; tables 4, 5). Detailed maps of participating jurisdictions are shown in the appendix (pp 178-208).

Coverage is now national in Australia, and contributions from additional registries increased the population coverage in another 14 of the 25 countries that participated in CONCORD-2 with subnational coverage. These are Africa: Algeria (from 1.6\% to 6.0\%); Central and South America: Brazil (from $5 \cdot 7 \%$ to $7 \cdot 7 \%$ ), Chile (from $5.5 \%$ to $13 \cdot 8 \%$ ), Colombia (from $6.9 \%$ to $9.0 \%$ ), and Ecuador (from $33.8 \%$ to $40 \cdot 2 \%$ ); North America: the USA (from $83.2 \%$ to $85.8 \%$ ); Asia: Japan (from $29 \cdot 2 \%$ to $40 \cdot 6 \%$ ), Thailand (from 5.9\% to $20 \cdot 3 \%$ ), and Turkey (from 5.4\% to $23 \cdot 4 \%$ ); Europe: France (from $18 \cdot 4 \%$ to $21 \cdot 7 \%$ ), Italy (from $38 \cdot 6 \%$ to $58 \cdot 3 \%$ ), Romania (from $3 \cdot 1 \%$ to $5 \cdot 0 \%$ ), Russia (from $0.9 \%$ to $5.6 \%$ ), and Switzerland (from $47.4 \%$ to $54.7 \%$ ); and Oceania: Australia (from $90 \cdot 8 \%$ to $100 \cdot 0 \%$. International coverage has been reduced by the loss of data from Indonesia (Jakarta) and from four countries in Africa: Gambia, Lesotho, Libya, and Tunisia.

Three of the Polish registries that participated in CONCORD-2 now use a different or anglicised name, changing the alphabetical order in the supplementary tables: Holy Cross (formerly Kielce), Lower Silesia (Wrocław), and Subcarpathia (Podkarpackie). All 16 voivodeships of Poland are now included.

Four registries submitted data with wider territorial coverage than before. The Burgundy (Digestive) registry in France submitted data for both the Saône-et-Loire and the Côte-d'Or departments; in Italy, the Biella registry now covers the Vercelli province as well as Biella, and the Milan registry now covers the Milan province and Lodi as well as the city of Milan; and the Cluj registry in Romania expanded coverage from Cluj county to include BistrițaNăsăud county.

We received more than 4700 datasets. We examined individual cancer registrations for 42222177 patients diagnosed with an index cancer during the period 2000-14 (table 3). Of these, 2690466 (6.4\%) were for an in-situ cancer, mostly of the cervix (54.6\% of 1708385 women), melanoma of the skin (27.0\% of 2262368 patients), breast
(10.6\% of 7379194 women), rectum $(4.8 \%$ of 1881039 patients), colon (4.4\% of 4619844 adults), or prostate $(0 \cdot 6 \%$ of 6069870 men; appendix pp 6-101). The proportions of in-situ cancer are not directly comparable between countries because some registries still do not record in-situ malignancies, whereas others did not submit data for cancers for which in-situ malignancy is common. The variation between continents is still of interest: for cervical cancer, it ranged from $2.2 \%$ in African registries to $23.6 \%$ in Central and South American registries, 37.4\% in Asian registries, $66.7 \%$ in European registries, and $81.9 \%$ in Oceania; US registries did not submit data for in-situ cervical cancers and only three Canadian provinces did so. The proportion of in-situ breast cancers varied from $0.2 \%$ in African registries to $4-6 \%$ in Central and South America, Asia, Europe, and Oceania, and $17.3 \%$ in North America (appendix pp 52-56).

Patients with in-situ cancer were not included in survival analyses. We excluded a further $227038(0 \cdot 5 \%)$ patients because the year of birth, the month or year of diagnosis, or the year of last known vital status was unknown; and $527408(1.2 \%)$ patients because the tumour was not a primary, invasive malignancy (behaviour code 3); or the morphology was that of Kaposi's sarcoma or lymphoma in a solid organ; or for other reasons (table 3). The proportion of records excluded for these reasons is shown for each cancer and each cancer registry in the appendix (pp 6-101).

Of the 38777265 patients otherwise eligible for inclusion in survival analyses, we excluded 1132833 (2.9\%) records because the cancer was registered only from a death certificate or discovered at autopsy (table 3 ) and a further $131407(0 \cdot 3 \%)$ for other reasons. These reasons included definite errors (unknown vital status, unknown sex, sexsite error, and invalid dates or sequence of dates) and possible errors, such as apparent inconsistencies between age, cancer site, and morphology (details on request). For example, we excluded hepatoblastomas in children older than 6 years and multiple myeloma in people aged less than 20 years, unless the record was confirmed as correct by the registry concerned.

Among the 37513025 patients available for survival analyses for all cancers combined $(96.7 \%$ of those eligible for inclusion), pathological evidence of malignancy (histology, cytology, or haematology) was available for 35502123 (94.6\%). This proportion ranged from $88.6 \%$ in Asia, $91.6 \%$ in Africa, and $92.4 \%$ in Central and South America up to $94-98 \%$ in Europe, Oceania, and North America (table 3). Continental variation was much wider for some cancers (appendix pp 6-101).

In what follows, we present results in a similar structure for each group of cancers. Differences between survival estimates are given as the arithmetic difference: for example, $12 \%$ is $2 \%$ (not $20 \%$ ) higher than $10 \%$. We use flags in the figures (figures 2,3) and tables (tables 6, 7) to indiate where survival estimates are based on national 


\begin{tabular}{|c|c|c|c|c|c|c|c|c|c|c|c|c|c|}
\hline & \multirow{2}{*}{$\begin{array}{l}\text { Calendar } \\
\text { period }\end{array}$} & \multirow{2}{*}{$\begin{array}{l}\text { Patients } \\
\text { submitted }\end{array}$} & \multicolumn{3}{|c|}{ Ineligible patients $\uparrow$} & \multirow{2}{*}{$\begin{array}{l}\text { Eligible } \\
\text { patients }\end{array}$} & \multicolumn{2}{|c|}{ Excludeds } & \multirow{2}{*}{$\begin{array}{l}\text { Patients } \\
\text { included }\end{array}$} & \multicolumn{4}{|c|}{ Data quality indicators $\mathbb{T}$} \\
\hline & & & $\begin{array}{l}\text { Incomplete } \\
\text { dates }\end{array}$ & In situ & Other & & DCO & Other & & $\begin{array}{l}\text { Microscop- } \\
\text { ically verified }\end{array}$ & $\begin{array}{l}\text { Non-specific } \\
\text { morphology }\end{array}$ & $\begin{array}{l}\text { Lost to } \\
\text { follow-up }\end{array}$ & Censored \\
\hline Africa & & 46627 & $9.6 \%$ & $0.4 \%$ & $1.1 \%$ & 41447 & $0.9 \%$ & $2 \cdot 1 \%$ & 40197 & $91.6 \%$ & $14.1 \%$ & $7.6 \%$ & $37.7 \%$ \\
\hline Algerian registries & 2000-14 & 18157 & $7.6 \%$ & $0.1 \%$ & $1.8 \%$ & 16434 & $1.8 \%$ & $3 \cdot 3 \%$ & 15602 & $98 \cdot 4 \%$ & $10.2 \%$ & $0.0 \%$ & $31.5 \%$ \\
\hline Mali (Bamako) & 2010-12 & 104 & $41 \cdot 3 \%$ & $0.0 \%$ & $0.0 \%$ & 61 & $0.0 \%$ & $1.6 \%$ & 60 & $100 \cdot 0 \%$ & $20 \cdot 0 \%$ & $0.0 \%$ & $0.0 \%$ \\
\hline Mauritius* & 2005-12 & 4125 & $0.0 \%$ & $0.0 \%$ & $0.4 \%$ & 4109 & $0.0 \%$ & $3.7 \%$ & 3959 & $96.7 \%$ & $19.8 \%$ & $0.0 \%$ & $2 \cdot 3 \%$ \\
\hline $\begin{array}{l}\text { Morocco } \\
\text { (Casablanca) }\end{array}$ & 2008-12 & 4840 & $1.4 \%$ & $0.0 \%$ & $0.1 \%$ & 4769 & $0.0 \%$ & $1 \cdot 8 \%$ & 4683 & $100 \cdot 0 \%$ & $2 \cdot 4 \%$ & $33.0 \%$ & $35 \cdot 6 \%$ \\
\hline Nigeria (Ibadan) & $2003-14$ & 11726 & $25 \cdot 4 \%$ & $1 \cdot 4 \%$ & $1 \cdot 2 \%$ & 8443 & $0.9 \%$ & $1 \cdot 1 \%$ & 8274 & $98.7 \%$ & $2.0 \%$ & $0.0 \%$ & $65 \cdot 3 \%$ \\
\hline $\begin{array}{l}\text { South Africa } \\
\text { (Eastern Cape) }\end{array}$ & 2000-14 & 7675 & $0.0 \%$ & $0.0 \%$ & $0.6 \%$ & 7631 & $0.0 \%$ & $0.2 \%$ & 7619 & $62 \cdot 3 \%$ & $39 \cdot 5 \%$ & $19 \cdot 7 \%$ & $40 \cdot 2 \%$ \\
\hline $\begin{array}{l}\text { America (Central and } \\
\text { South) }\end{array}$ & & 906076 & $5 \cdot 4 \%$ & $3 \cdot 1 \%$ & $0.7 \%$ & 822687 & $13 \cdot 7 \%$ & $1 \cdot 1 \%$ & 700946 & $92 \cdot 4 \%$ & $8.0 \%$ & $5 \cdot 2 \%$ & $3.7 \%$ \\
\hline $\begin{array}{l}\text { Argentinian } \\
\text { registries } \neq\end{array}$ & $2000-14$ & 75167 & $1.7 \%$ & $1.5 \%$ & $0.5 \%$ & 72366 & $10 \cdot 8 \%$ & $0.6 \%$ & 64151 & $96.5 \%$ & $5.7 \%$ & $0.0 \%$ & $2 \cdot 3 \%$ \\
\hline Brazilian registries & 2000-14 & 191344 & $18.5 \%$ & $3.9 \%$ & $0.5 \%$ & 147622 & $8.0 \%$ & $0.9 \%$ & 134597 & $90 \cdot 0 \%$ & $10.6 \%$ & $22.9 \%$ & $0.3 \%$ \\
\hline Chilean registries & $2000-12$ & 28987 & $0.0 \%$ & $0.8 \%$ & $0.7 \%$ & 28555 & $7.6 \%$ & $0.1 \%$ & 26363 & $86 \cdot 2 \%$ & $12.0 \%$ & $0.0 \%$ & $13 \cdot 6 \%$ \\
\hline $\begin{array}{l}\text { Colombian } \\
\text { registries }\end{array}$ & $2000-14$ & 63402 & $3 \cdot 1 \%$ & $1.5 \%$ & $1 \cdot 2 \%$ & 59740 & $5.0 \%$ & $0.9 \%$ & 56245 & $89.9 \%$ & $11 \cdot 3 \%$ & $0.0 \%$ & $21.0 \%$ \\
\hline Costa Rica* & $2002-14$ & 72900 & $0.0 \%$ & $4 \cdot 1 \%$ & $1.4 \%$ & 68900 & $8 \cdot 4 \%$ & $0.8 \%$ & 62536 & $90 \cdot 1 \%$ & $13.0 \%$ & $0.0 \%$ & $0.0 \%$ \\
\hline Cuba* & 2000-12 & 193196 & $0.0 \%$ & $0.0 \%$ & $0.2 \%$ & 192755 & $32 \cdot 3 \%$ & $2 \cdot 5 \%$ & 125696 & $91.8 \%$ & $5 \cdot 1 \%$ & $2.6 \%$ & $0.0 \%$ \\
\hline $\begin{array}{l}\text { Ecuadorian } \\
\text { registries }\end{array}$ & $2000-14$ & 71798 & $7 \cdot 7 \%$ & $8 \cdot 2 \%$ & $0.8 \%$ & 59892 & $9.8 \%$ & $1.6 \%$ & 53043 & $92.0 \%$ & $9.9 \%$ & $0.3 \%$ & $2 \cdot 7 \%$ \\
\hline Guadeloupe* & 2008-13 & 8896 & $0.0 \%$ & $12.0 \%$ & $0.3 \%$ & 7802 & $0.0 \%$ & $0.2 \%$ & 7787 & $99 \cdot 1 \%$ & $2 \cdot 1 \%$ & $0.0 \%$ & $57 \cdot 7 \%$ \\
\hline Martinique* & $2000-12$ & 16066 & $0.0 \%$ & $0.0 \%$ & $0.1 \%$ & 16053 & $0.0 \%$ & $1.7 \%$ & 15779 & $97 \cdot 3 \%$ & $0.7 \%$ & $7.3 \%$ & $0.1 \%$ \\
\hline $\begin{array}{l}\text { Mexico } \\
\text { (childhood) } \ddagger\end{array}$ & 2008-14 & 9749 & $5.8 \%$ & $0.0 \%$ & $9.7 \%$ & 8236 & $0.0 \%$ & $0.5 \%$ & 8194 & $99.8 \%$ & $3.9 \%$ & $9 \cdot 3 \%$ & $7.6 \%$ \\
\hline Peru (Lima) & 2010-12 & 19078 & $0.1 \%$ & $0.0 \%$ & $0.7 \%$ & 18929 & $8.9 \%$ & $0.1 \%$ & 17226 & $93.9 \%$ & $2.9 \%$ & $0.0 \%$ & $10 \cdot 2 \%$ \\
\hline Puerto Rico* & 2000-11 & 118877 & $3 \cdot 7 \%$ & $3.9 \%$ & $0.7 \%$ & 109001 & $6 \cdot 4 \%$ & $0.3 \%$ & 101613 & $98 \cdot 4 \%$ & $3 \cdot 4 \%$ & $0.0 \%$ & $0.0 \%$ \\
\hline Uruguay* & 2008-12 & 36616 & $0.0 \%$ & $9 \cdot 6 \%$ & $0.7 \%$ & 32836 & $15 \cdot 5 \%$ & $0.1 \%$ & 27716 & $85 \cdot 0 \%$ & $15 \cdot 9 \%$ & $0.0 \%$ & $0.0 \%$ \\
\hline America (North) & & 15925870 & $0.7 \%$ & $6.8 \%$ & $0.7 \%$ & 14622183 & $1.8 \%$ & $0 \cdot 3 \%$ & 14320034 & $97 \cdot 7 \%$ & $3.0 \%$ & $1.4 \%$ & $0.0 \%$ \\
\hline Canadian registries & 2000-14 & 1519461 & $0.1 \%$ & $4.9 \%$ & $0.7 \%$ & 1431975 & $1 \cdot 2 \%$ & $0.4 \%$ & 1409413 & $94.8 \%$ & $5 \cdot 5 \%$ & $0.0 \%$ & $0.0 \%$ \\
\hline US registries & 2000-14 & 14406409 & $0.7 \%$ & $7.0 \%$ & $0.7 \%$ & 13190208 & $1.8 \%$ & $0.3 \%$ & 12910621 & $98.0 \%$ & $2.8 \%$ & $1.5 \%$ & $0.0 \%$ \\
\hline Asia & & 6595363 & $0.6 \%$ & $3 \cdot 4 \%$ & $0.4 \%$ & 6298518 & $4.7 \%$ & $0 \cdot 4 \%$ & 5976959 & $88.6 \%$ & $11 \cdot 5 \%$ & $0.4 \%$ & $1.0 \%$ \\
\hline Chinese registries & $2003-13$ & 610729 & $0.8 \%$ & $0.2 \%$ & $0.2 \%$ & 603861 & $1.4 \%$ & $0.1 \%$ & 594533 & $66 \cdot 2 \%$ & $41.8 \%$ & $3 \cdot 2 \%$ & $0 \cdot 1 \%$ \\
\hline Cyprus* & 2004-14 & 25086 & $1.4 \%$ & $2 \cdot 6 \%$ & $0.8 \%$ & 23880 & $9.0 \%$ & $0.5 \%$ & 21610 & $98.9 \%$ & $1.8 \%$ & $0.0 \%$ & $34.8 \%$ \\
\hline Hong Kong* & 2005-14 & 78127 & $3.8 \%$ & $0.0 \%$ & $0.0 \%$ & 75146 & $0.4 \%$ & $0.2 \%$ & 74721 & $96.6 \%$ & $0.0 \%$ & $5.5 \%$ & $0.0 \%$ \\
\hline Indian registries & 2000-14 & 5048 & $3 \cdot 2 \%$ & $0.0 \%$ & $0.0 \%$ & 4882 & $1.7 \%$ & $0.6 \%$ & 4774 & $82 \cdot 1 \%$ & $25 \cdot 1 \%$ & $1.8 \%$ & $0 \cdot 1 \%$ \\
\hline Iran (Golestan) & $2006-08$ & 1187 & $0.0 \%$ & $0.0 \%$ & $0.5 \%$ & 1181 & $8.9 \%$ & $3 \cdot 1 \%$ & 1039 & $82 \cdot 1 \%$ & $17 \cdot 9 \%$ & $8.9 \%$ & $0.0 \%$ \\
\hline $\mid$ |srael* & $2000-13$ & 282191 & $0.0 \%$ & $7 \cdot 3 \%$ & $2 \cdot 2 \%$ & 255359 & $4.8 \%$ & $0 \cdot 4 \%$ & 241881 & $96 \cdot 8 \%$ & $4 \cdot 2 \%$ & $0.0 \%$ & $0.0 \%$ \\
\hline Japanese registries & 2000-14 & 2237861 & $1.0 \%$ & $4.8 \%$ & $0.5 \%$ & 2096697 & $12 \cdot 4 \%$ & $0.1 \%$ & 1834894 & $91 \cdot 4 \%$ & $11 \cdot 3 \%$ & $0.0 \%$ & $1.7 \%$ \\
\hline Jordan* & 2000-14 & 43442 & $0.2 \%$ & $1.2 \%$ & $1.5 \%$ & 42179 & $0.2 \%$ & $1.6 \%$ & 41433 & $99 \cdot 1 \%$ & $3.0 \%$ & $5.9 \%$ & $0.0 \%$ \\
\hline Korea* & 2000-14 & 1770463 & $0.5 \%$ & $0.0 \%$ & $0.0 \%$ & 1762176 & $0.0 \%$ & $0.1 \%$ & 1760804 & $93 \cdot 1 \%$ & $7.8 \%$ & $0.0 \%$ & $0.0 \%$ \\
\hline Kuwait* & $2000-13$ & 8931 & $0.0 \%$ & $1 \cdot 4 \%$ & $1 \cdot 1 \%$ & 8710 & $2 \cdot 3 \%$ & $0.3 \%$ & 8484 & $99.8 \%$ & $0.4 \%$ & $1 \cdot 2 \%$ & $0.0 \%$ \\
\hline Malaysia (Penang) & $2000-13$ & 19612 & $0.3 \%$ & $0.0 \%$ & $0.1 \%$ & 19527 & $1.6 \%$ & $2 \cdot 1 \%$ & 18805 & $94 \cdot 2 \%$ & $9 \cdot 5 \%$ & $0.0 \%$ & $13.0 \%$ \\
\hline Mongolia* & 2003-14 & 1025 & $0.0 \%$ & $1.1 \%$ & $0.0 \%$ & 1014 & $0.3 \%$ & $1 \cdot 2 \%$ & 999 & $77 \cdot 0 \%$ & $4 \cdot 1 \%$ & $7.6 \%$ & $0.0 \%$ \\
\hline Qatar* & 2000-14 & 7940 & $0.0 \%$ & $1.0 \%$ & $1.0 \%$ & 7778 & $1.0 \%$ & $0.7 \%$ & 7642 & $95 \cdot 4 \%$ & $6 \cdot 3 \%$ & $0.0 \%$ & $51.0 \%$ \\
\hline Singapore* & 2000-14 & 122461 & $0.0 \%$ & $7.0 \%$ & $1.9 \%$ & 111495 & $1.1 \%$ & $0.3 \%$ & 109992 & $91 \cdot 7 \%$ & $1.9 \%$ & $0.0 \%$ & $0.0 \%$ \\
\hline Taiwan* & 2000-14 & 941313 & $0.1 \%$ & $8.6 \%$ & $0.1 \%$ & 859169 & $0.0 \%$ & $0.1 \%$ & 858683 & $86 \cdot 6 \%$ & $0.5 \%$ & $0.0 \%$ & $0.0 \%$ \\
\hline Thai registries & 2000-14 & 183776 & $0.0 \%$ & $0.3 \%$ & $0.5 \%$ & 182455 & $3.8 \%$ & $8.7 \%$ & 159528 & $68.6 \%$ & $34.0 \%$ & $0.0 \%$ & $3.0 \%$ \\
\hline \multirow[t]{2}{*}{ Turkish registries } & $2000-13$ & 256171 & $1.5 \%$ & $2 \cdot 7 \%$ & $0.9 \%$ & 243009 & $1.9 \%$ & $0.5 \%$ & 237137 & $94 \cdot 7 \%$ & $7.9 \%$ & $0.2 \%$ & $3.8 \%$ \\
\hline & & & & & & & & & & & \multicolumn{3}{|c|}{ (Table 3 continues on next page } \\
\hline
\end{tabular}


population coverage, are unstandardised, or are considered less reliable. Where relevant, we mention in the text only reliable, age-standardised survival estimates. Where possible, we also present graphics of national trends in cancer survival over the 20-year period 1995-2014 Estimates for patients diagnosed during 1995-99 are for countries that provided data for one of the ten cancers included in CONCORD-2. ${ }^{\circ}$

\begin{tabular}{|c|c|c|c|c|c|c|c|c|c|c|c|c|c|}
\hline & \multirow{2}{*}{$\begin{array}{l}\text { Calendar } \\
\text { period }\end{array}$} & \multirow{2}{*}{$\begin{array}{l}\text { Patients } \\
\text { submitted }\end{array}$} & \multicolumn{3}{|c|}{ Ineligible patients $\dagger$} & \multirow{2}{*}{$\begin{array}{l}\text { Eligible } \\
\text { patients }\end{array}$} & \multicolumn{2}{|c|}{ Excluded $\$$} & \multirow{2}{*}{$\begin{array}{l}\text { Patients } \\
\text { included }\end{array}$} & \multicolumn{4}{|c|}{ Data quality indicators $\Phi$} \\
\hline & & & $\begin{array}{l}\text { Incomplete } \\
\text { dates }\end{array}$ & In situ & Other & & DCO & Other & & $\begin{array}{l}\text { Microscop- } \\
\text { ically verified }\end{array}$ & $\begin{array}{l}\text { Non-specific } \\
\text { morphology }\end{array}$ & $\begin{array}{l}\text { Lost to } \\
\text { follow-up }\end{array}$ & Censored \\
\hline \multicolumn{14}{|c|}{ (Continued from previous page) } \\
\hline Europe & & 17057088 & $0.1 \%$ & $7.0 \%$ & $2 \cdot 1 \%$ & 15481564 & $2 \cdot 8 \%$ & $0.3 \%$ & 14991316 & $94.0 \%$ & $3.9 \%$ & $0.9 \%$ & $2 \cdot 1 \%$ \\
\hline Austria* & 2000-14 & 486379 & $0.0 \%$ & $7 \cdot 4 \%$ & $1.2 \%$ & 444735 & $6 \cdot 1 \%$ & $1 \cdot 1 \%$ & 412683 & $98.0 \%$ & $4.9 \%$ & $0.0 \%$ & $0.0 \%$ \\
\hline $\begin{array}{l}\text { Belarus } \\
\text { (childhood) } \ddagger\end{array}$ & $2000-14$ & 1740 & $0.0 \%$ & $0.0 \%$ & $0.0 \%$ & 1740 & $0.6 \%$ & $2 \cdot 5 \%$ & 1687 & $97 \cdot 9 \%$ & $2 \cdot 5 \%$ & $1.5 \%$ & $0.0 \%$ \\
\hline Belgium* & 2004-14 & 616737 & $0.0 \%$ & $11 \cdot 4 \%$ & $0.2 \%$ & 545373 & $0.0 \%$ & $0.2 \%$ & 544110 & $98 \cdot 4 \%$ & $2.0 \%$ & $1.1 \%$ & $0.0 \%$ \\
\hline Bulgaria* & $2000-14$ & 299563 & $0.0 \%$ & $0.0 \%$ & $0.1 \%$ & 299333 & $8.5 \%$ & $0.0 \%$ & 273868 & $89 \cdot 2 \%$ & $1.4 \%$ & $0.1 \%$ & $0.0 \%$ \\
\hline Croatia* $^{*}$ & 2000-14 & 246883 & $0.0 \%$ & $3 \cdot 5 \%$ & $0.2 \%$ & 237793 & $6 \cdot 2 \%$ & $0.1 \%$ & 222776 & $82.9 \%$ & $0.5 \%$ & $0.0 \%$ & $0.0 \%$ \\
\hline Czech Republic* & 2000-14 & 640594 & $0.0 \%$ & $7 \cdot 5 \%$ & $1.6 \%$ & 582748 & $1 \cdot 3 \%$ & $0.4 \%$ & 572368 & $90 \cdot 3 \%$ & $1.5 \%$ & $0.0 \%$ & $0.0 \%$ \\
\hline Denmark* & 2000-14 & 366310 & $0.0 \%$ & $0.0 \%$ & $0 \cdot 2 \%$ & 365525 & $0.0 \%$ & $0.1 \%$ & 365105 & $96 \cdot 3 \%$ & $6 \cdot 8 \%$ & $0.2 \%$ & $0.0 \%$ \\
\hline Estonia* & $2000-12$ & 64038 & $0.0 \%$ & $1.8 \%$ & $0.7 \%$ & 62396 & $3.9 \%$ & $0 \cdot 2 \%$ & 59848 & $89 \cdot 2 \%$ & $2.0 \%$ & $0.3 \%$ & $0.0 \%$ \\
\hline Finland* & 2000-14 & 328513 & $0.6 \%$ & $5 \cdot 4 \%$ & $0.9 \%$ & 306077 & $3.8 \%$ & $0.1 \%$ & 294268 & $95 \cdot 8 \%$ & $3 \cdot 2 \%$ & $0.1 \%$ & $0.0 \%$ \\
\hline French registries $\ddagger$ & $2000-12$ & 466020 & $0.2 \%$ & $0.0 \%$ & $0.3 \%$ & 463588 & $0.0 \%$ & $0.6 \%$ & 460927 & $96 \cdot 1 \%$ & $0.6 \%$ & $1.8 \%$ & $0.1 \%$ \\
\hline German registries & 2000-14 & 1925070 & $0.4 \%$ & $4.5 \%$ & $1.0 \%$ & 1811465 & $10 \cdot 3 \%$ & $0.2 \%$ & 1621312 & $97.5 \%$ & $0.7 \%$ & $0.3 \%$ & $17 \cdot 7 \%$ \\
\hline Gibraltar* & $2000-10$ & 732 & $13 \cdot 0 \%$ & $11 \cdot 7 \%$ & $1.1 \%$ & 543 & $0.2 \%$ & $1.7 \%$ & 533 & $99.6 \%$ & $0.8 \%$ & $0.0 \%$ & $41 \cdot 7 \%$ \\
\hline Greece (childhood) $\ddagger$ & 2000-14 & 1743 & $0.6 \%$ & $0.0 \%$ & $0.0 \%$ & 1733 & $0.0 \%$ & $0.4 \%$ & 1726 & $99.9 \%$ & $0.1 \%$ & $0.8 \%$ & $0.2 \%$ \\
\hline Iceland* & $2000-14$ & 15245 & $0.0 \%$ & $1.4 \%$ & $0.8 \%$ & 14918 & $0.8 \%$ & $0.1 \%$ & 14782 & $96 \cdot 5 \%$ & $3.9 \%$ & $0.0 \%$ & $0.0 \%$ \\
\hline Ireland* & $2000-13$ & 240962 & $0.0 \%$ & $16 \cdot 3 \%$ & $0.9 \%$ & 199552 & $1.5 \%$ & $0.2 \%$ & 196331 & $92 \cdot 2 \%$ & $1.7 \%$ & $0.0 \%$ & $0.0 \%$ \\
\hline Italian registries & 2000-14 & 1452003 & $0.0 \%$ & $1.8 \%$ & $0.8 \%$ & 1414476 & $0.7 \%$ & $0.3 \%$ & 1400117 & $87 \cdot 5 \%$ & $14 \cdot 1 \%$ & $0.7 \%$ & $0.8 \%$ \\
\hline Latvia* & 2000-14 & 97852 & $0.0 \%$ & $0.1 \%$ & $26 \cdot 8 \%$ & 71511 & $0.0 \%$ & $0.6 \%$ & 71082 & $99.8 \%$ & $1.1 \%$ & $0.0 \%$ & $0.0 \%$ \\
\hline Lithuania* & $2000-12$ & 154857 & $0.0 \%$ & $4 \cdot 1 \%$ & $1.1 \%$ & 146896 & $4.9 \%$ & $0.2 \%$ & 139475 & $87.6 \%$ & $1.5 \%$ & $0.0 \%$ & $0.3 \%$ \\
\hline Malta* & 2000-13 & 17625 & $0.0 \%$ & $6 \cdot 9 \%$ & $1.8 \%$ & 16091 & $3 \cdot 1 \%$ & $0.4 \%$ & 15518 & $92 \cdot 4 \%$ & $8.9 \%$ & $0.0 \%$ & $0.0 \%$ \\
\hline Netherlands* & 2000-14 & 1047456 & $0.0 \%$ & $3 \cdot 8 \%$ & $1 \cdot 2 \%$ & 994826 & $0.2 \%$ & $0.6 \%$ & 987029 & $96 \cdot 2 \%$ & $4.0 \%$ & $0.5 \%$ & $0.0 \%$ \\
\hline Norway* & 2000-14 & 488733 & $0.0 \%$ & $10 \cdot 3 \%$ & $32 \cdot 5 \%$ & 279696 & $0.5 \%$ & $0 \cdot 1 \%$ & 277991 & $99 \cdot 8 \%$ & $0.4 \%$ & $0.2 \%$ & $0.0 \%$ \\
\hline Poland* & 2000-14 & 1389978 & $0.0 \%$ & $0.1 \%$ & $0 \cdot 3 \%$ & 1383780 & $2.5 \%$ & $0.3 \%$ & 1344837 & $91 \cdot 4 \%$ & $1.3 \%$ & $0.0 \%$ & $0.0 \%$ \\
\hline Portugal* & 2000-14 & 408523 & $0.7 \%$ & $2 \cdot 7 \%$ & $1.5 \%$ & 388199 & $0.1 \%$ & $0.2 \%$ & 386853 & $96 \cdot 7 \%$ & $2.7 \%$ & $1.7 \%$ & $0.0 \%$ \\
\hline Romania (Cluj) & $2006-12$ & 17740 & $0.0 \%$ & $3 \cdot 1 \%$ & $1.7 \%$ & 16894 & $16 \cdot 6 \%$ & $0.2 \%$ & 14060 & $90 \cdot 1 \%$ & $10 \cdot 7 \%$ & $0.0 \%$ & $0.0 \%$ \\
\hline Russian registries & 2000-14 & 252171 & $0.0 \%$ & $0.5 \%$ & $0.4 \%$ & 249928 & $0.8 \%$ & $1.0 \%$ & 245591 & $85 \cdot 4 \%$ & $4 \cdot 1 \%$ & $2.0 \%$ & $1 \cdot 3 \%$ \\
\hline Slovakia* & 2000-10 & 180029 & $0.0 \%$ & $4 \cdot 1 \%$ & $1 \cdot 3 \%$ & 170269 & $8.2 \%$ & $0 \cdot 1 \%$ & 156122 & $94.0 \%$ & $6.7 \%$ & $0.0 \%$ & $0.0 \%$ \\
\hline Slovenia* & $2000-13$ & 124213 & $0.0 \%$ & $13 \cdot 0 \%$ & $2 \cdot 4 \%$ & 105052 & $2.0 \%$ & $0.0 \%$ & 102970 & $93 \cdot 5 \%$ & $0.3 \%$ & $0.1 \%$ & $0.0 \%$ \\
\hline Spanish registries & 2000-14 & 417865 & $0.3 \%$ & $6.8 \%$ & $0.9 \%$ & 384586 & $1.9 \%$ & $0.2 \%$ & 376759 & $91.7 \%$ & $2 \cdot 2 \%$ & $0.5 \%$ & $0.5 \%$ \\
\hline Sweden* & 2000-14 & 676693 & $0.0 \%$ & $15 \cdot 4 \%$ & $3.0 \%$ & 551717 & $1.1 \%$ & $0 \cdot 2 \%$ & 544531 & $98.6 \%$ & $2 \cdot 2 \%$ & $0.2 \%$ & $0.0 \%$ \\
\hline Swiss registriesł & 2000-14 & 241610 & $0.0 \%$ & $7 \cdot 9 \%$ & $2 \cdot 5 \%$ & 216439 & $1.2 \%$ & $0.5 \%$ & 212695 & $95.9 \%$ & $2 \cdot 4 \%$ & $4.6 \%$ & $3.9 \%$ \\
\hline$U K^{*}$ & 2000-14 & 4389211 & $0.1 \%$ & $13.0 \%$ & $1.4 \%$ & 3753685 & $1.8 \%$ & $0.3 \%$ & 3673362 & $94.9 \%$ & $4.5 \%$ & $1.9 \%$ & $0.0 \%$ \\
\hline Oceania & & 1691153 & $0.3 \%$ & $9 \cdot 7 \%$ & $0.7 \%$ & 1510866 & $1.6 \%$ & $0 \cdot 2 \%$ & 1483573 & $96 \cdot 5 \%$ & $3 \cdot 6 \%$ & $0.0 \%$ & $0.0 \%$ \\
\hline Australia* & 2000-14 & 1443620 & $0.3 \%$ & $11 \cdot 3 \%$ & $0.8 \%$ & 1263961 & $1 \cdot 4 \%$ & $0 \cdot 2 \%$ & 1244350 & $97 \cdot 0 \%$ & $3.0 \%$ & $0.0 \%$ & $0.0 \%$ \\
\hline New Zealand* & 2000-14 & 247533 & $0.0 \%$ & $0.0 \%$ & $0 \cdot 3 \%$ & 246905 & $2.9 \%$ & $0 \cdot 2 \%$ & 239223 & $94 \cdot 3 \%$ & $6 \cdot 3 \%$ & $0.0 \%$ & $0.0 \%$ \\
\hline Total & & 42222177 & $0.5 \%$ & $6 \cdot 4 \%$ & $1.2 \%$ & 38777265 & $2.9 \%$ & $0.3 \%$ & 37513025 & $94.6 \%$ & $4.9 \%$ & $1.0 \%$ & $1.1 \%$ \\
\hline $\begin{array}{l}\text { DCO=death certificate on } \\
\text { of last known vital status } \\
\text { data or for tumours that } \\
\text { metastatic (behavior cod } \\
\text { malignancies only. SDCO: } \\
\text { submitted for CONCORD- } \\
\text { TNon-specific morpholos } \\
\text { diagnosis or, if diagnosed }\end{array}$ & $\begin{array}{l}\text { y. }{ }^{*} \text { Data with } \\
\text { is unknown. } \\
\text { re benign (b } \\
\text { 9); or for pa } \\
\text { tumours reg } \\
\text { 3. Other: vita } \\
\text { (solid tum } \\
\text { in } 2010 \text { or la }\end{array}$ & $\begin{array}{l}\text { 100\% coverag } \\
\text { In-situ maligna } \\
\text { ehaviour code } \\
\text { atients with age } \\
\text { istered only fro } \\
\text { al status or sex } \\
\text { ours only): ICD- } \\
\text { ter, before Dec }\end{array}$ & $\begin{array}{l}\text { e of the nationa } \\
\text { ncy (ICD-O-3 be } \\
\text {, except brain } \mathrm{t} \\
\text { outside the ran } \\
\mathrm{n} \text { a death certif } \\
\text { inknown; invali } \\
0-3^{17,18} \text { morphol } \\
31,2014 \text {. }\end{array}$ & $\begin{array}{l}\text { al populati } \\
\text { ehaviour c } \\
\text { umours), } \\
\text { ige } 0-14 \text { ye } \\
\text { ficate or de } \\
\text { id date or s } \\
\text { logy code i }\end{array}$ & 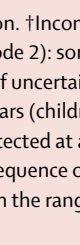 & $\begin{array}{l}\text { lete dates: reco } \\
\text { registries do n } \\
\text { ehaviour (beh } \\
\text { ) or 15-99 yea } \\
\text { opsy. Sweden } \\
\text { ates; inconsist } \\
\text { 3000-8005. C }\end{array}$ & $\begin{array}{l}\text { rds in whi } \\
\text { ot registe } \\
\text { avior code } \\
\text { s (adults) } \\
\text { does not } r \\
\text { ency of se } \\
\text { nsored: } p\end{array}$ & $\begin{array}{l}\text { in the ye } \\
\text { in-situ c } \\
\text { 1), meta } \\
\text { or other } \\
\text { gister D } \\
\text {-site, sit } \\
\text { tients w }\end{array}$ & $\begin{array}{l}\text { birth is unkn } \\
\text { ers; other regi } \\
\text { ic from anoth } \\
\text { ditions. } \neq \text { Dat } \\
\text {; autopsy-det } \\
\text { orphology, ag } \\
\text { last known v }\end{array}$ & $\begin{array}{l}\text {; or the month } \\
\text { s did not submi } \\
\text { rgan (behavior } \\
\text { th } 100 \% \text { coveras } \\
\text { d cases were not } \\
\text { te, age-morpho } \\
\text { status is "alive" }\end{array}$ & $\begin{array}{l}\text { ir year of diagn } \\
\text { them. Other: } r \\
\text { ode } 6 \text { ), or unkr } \\
\text { e of the nation } \\
\text { submitted for } \\
\text { ogy, or age-sit } \\
\text { and who were } c\end{array}$ & $\begin{array}{l}\text { is is unknow } \\
\text { cords with in } \\
\text { wn if priman } \\
\text { population f } \\
\text { ONCORD-2 b } \\
\text { morphology } \\
\text { nsored withir }\end{array}$ & $\begin{array}{l}n \text {; or the yea } \\
\text { complete } \\
\text { y or } \\
\text { or childhooc } \\
\text { ut have beer } \\
5 \text { years of }\end{array}$ \\
\hline
\end{tabular}




\section{Oesophagus}

Results are available for 734428 adults from 290 registries in 60 countries (table 2, table 4). In 2010-14, 5-year age-standardised net survival was in the range $10-30 \%$ in most countries, with a much wider range in Asia (appendix p 248). Most survival estimates were considered reliable (table 6; appendix pp 138-151).

\begin{tabular}{|c|c|c|c|c|c|c|c|c|c|c|c|}
\hline & \multirow{2}{*}{$\begin{array}{l}\text { Population } \\
\text { covered }\end{array}$} & \multirow{2}{*}{$\begin{array}{l}\text { Percentage } \\
\text { of national } \\
\text { population } \\
\text { covered }\end{array}$} & \multicolumn{8}{|c|}{ Number of patients } & \multirow{2}{*}{$\begin{array}{l}\text { Total } \\
\text { number of } \\
\text { patients } \dagger\end{array}$} \\
\hline & & & Oesophagus & Stomach & Colon & Rectum & Liver & Pancreas & Lung & $\begin{array}{l}\text { Melanoma } \\
\text { of the skin }\end{array}$ & \\
\hline Africa & 10533762 & $3.5 \%$ & 3057 & 1731 & 2172 & 1487 & 869 & 379 & 2734 & 368 & 40197 \\
\hline Algerian registries & 2447075 & $6 \cdot 3 \%$ & 104 & 1129 & 1331 & 827 & 244 & 272 & 1852 & 248 & 15602 \\
\hline Mali (Bamako) & 764245 & $9.0 \%$ &.. & .. &.. & .. &.. & .. & .. &.. & 60 \\
\hline Mauritius* & 1268567 & $100 \cdot 0 \%$ & 59 & 494 & 494 & 289 & 27 & 45 & 584 & 4 & 3959 \\
\hline Morocco (Casablanca) & 2178083 & $12 \cdot 7 \%$ &.. & .. & .. & .. & .. & .. & .. &.. & 4683 \\
\hline Nigeria (Ibadan) & 2797220 & $1.6 \%$ &.. & .. & 230 & 266 & 333 & .. & .. & 69 & 8274 \\
\hline $\begin{array}{l}\text { South Africa (Eastern } \\
\text { Cape) }\end{array}$ & 1078572 & $2.0 \%$ & 2894 & 108 & 117 & 105 & 265 & 62 & 298 & 47 & 7619 \\
\hline $\begin{array}{l}\text { America (Central and } \\
\text { South) }\end{array}$ & 99818363 & $23 \cdot 7 \%$ & 13528 & 60643 & 82843 & 26424 & 9019 & 15731 & 53959 & 8202 & 700946 \\
\hline Argentinian registriesł & 3973922 & $9 \cdot 2 \%$ & 1152 & 2686 & 6076 & 1959 & 968 & 1657 & 5195 & 1084 & 64151 \\
\hline Brazilian registries & 15882624 & $7 \cdot 7 \%$ & 6678 & 15567 & 21984 & 10354 & 858 & 5520 & 4884 & 1424 & 134597 \\
\hline Chilean registries & 2459133 & $13 \cdot 8 \%$ & 918 & 4423 & 1949 & 824 & 525 & 793 & 2166 & 554 & 26363 \\
\hline Colombian registries & 4277369 & $9.0 \%$ & 642 & 7988 & 3874 & 1990 & 1129 & 1303 & 3965 & 1373 & 56245 \\
\hline Costa Rica* & 4757606 & $100 \cdot 0 \%$ & 528 & 8577 & 5438 & 1926 & 1239 & 1188 & 2688 & 1432 & 62536 \\
\hline Cuba* & 11379111 & $100 \cdot 0 \%$ &.. & 6664 & 15047 & .. &.. & .. & 19344 &.. & 125696 \\
\hline Ecuadorian registries & 6398546 & $40 \cdot 2 \%$ & 486 & 7210 & 3203 & 1622 & 1502 & 1069 & 2641 & 1080 & 53043 \\
\hline Guadeloupe* & 450273 & $100 \cdot 0 \%$ & 119 & 521 & 724 & 210 & 82 & 167 & 308 & 52 & 7787 \\
\hline Martinique* & 396425 & $100 \cdot 0 \%$ & 213 & 973 & 1305 & 423 & 206 & 473 & 693 & 164 & 15779 \\
\hline Mexico (childhood) $\ddagger$ & 35188624 & $100 \cdot 0 \%$ &.. & .. &.. &.. &.. & .. & .. &.. & 8194 \\
\hline Peru (Lima) & 7548697 & $24 \cdot 4 \%$ &.. & .. & 2803 & 992 &.. & .. & .. &.. & 17226 \\
\hline Puerto Rico* & 3686517 & $100 \cdot 0 \%$ & 1743 & 3900 & 14594 & 3882 & 2202 & 1984 & 6570 & 1039 & 101613 \\
\hline Uruguay* & 3419516 & $100 \cdot 0 \%$ & 1049 & 2134 & 5846 & 2242 & 308 & 1577 & 5505 &.. & 27716 \\
\hline America (North) & 301237785 & $84.8 \%$ & 195255 & 283566 & 1471548 & 461707 & 246966 & 462187 & 2564507 & 678206 & 14320034 \\
\hline Canadian registries & 27213277 & $76 \cdot 5 \%$ & 18788 & 33889 & 166760 & 60958 & 20858 & 41908 & 236434 & 65235 & 1409413 \\
\hline US registries & 274024508 & $85 \cdot 8 \%$ & 176467 & 249677 & 1304788 & 400749 & 226108 & 420279 & 2328073 & 612971 & 12910621 \\
\hline Asia & 227771765 & $7 \cdot 2 \%$ & 206254 & 971935 & 703081 & 361987 & 617479 & 205345 & 950362 & 31314 & 5976959 \\
\hline Chinese registries & 31755347 & $2 \cdot 3 \%$ & 72714 & 92578 & 35001 & 31498 & 74295 & 19110 & 133932 & 1449 & 594533 \\
\hline Cyprus* & 1153658 & $100 \cdot 0 \%$ & 81 & 802 & 2665 & 788 & 247 & 534 & 2489 & 589 & 21610 \\
\hline Hong Kong* & 7241700 & $100 \cdot 0 \%$ &.. & .. & 28797 & 12856 &.. & .. & .. &.. & 74721 \\
\hline Indian registries & 1005294 & $0.1 \%$ & 269 & 303 & 199 & 191 & 230 & 134 & 850 & 29 & 4774 \\
\hline Iran (Golestan) & 1893646 & $2 \cdot 4 \%$ & 412 & 353 & 216 & 58 &.. & .. & .. &.. & 1039 \\
\hline |srael* & 7939483 & $100 \cdot 0 \%$ & 1691 & 9737 & 33938 & 9401 & 2310 & 8083 & 25347 & 12265 & 241881 \\
\hline Japanese registries & 51445407 & $40 \cdot 6 \%$ & 63631 & 381457 & 247682 & 102776 & 122792 & 79636 & 276444 & 4018 & 1834894 \\
\hline Jordan* & 7416083 & $100 \cdot 0 \%$ & 352 & 1955 & 5116 & 1775 & 606 & 952 & 4282 & 214 & 41433 \\
\hline Korea* & 50074400 & $100 \cdot 0 \%$ & 30627 & 396213 & 187078 & 121053 & 214821 & 59357 & 257345 & 5771 & 1760804 \\
\hline Kuwait* & 3753121 & $100 \cdot 0 \%$ & 90 & 207 & 908 & 330 & 261 & 240 & 559 & 18 & 8484 \\
\hline Malaysia (Penang) & 1543500 & $5 \cdot 2 \%$ & 290 & 1061 & 2285 & 1126 & 927 & 539 & 2863 &.. & 18805 \\
\hline Mongolia* & 1468823 & $100 \cdot 0 \%$ &. & .. &.. &.. &. & .. & .. &.. & 999 \\
\hline Qatar* & 2172065 & $100 \cdot 0 \%$ & 98 & 361 & 784 & 283 & 438 & 186 & 587 & 55 & 7642 \\
\hline Singapore* & 3870700 & $100 \cdot 0 \%$ & 1434 & 6822 & 17225 & 6241 & 7101 & 3481 & 17921 & 367 & 109992 \\
\hline Taiwan* & 23123866 & $100 \cdot 0 \%$ & 27680 & 54983 & 108844 & 57163 & 158157 & 22283 & 141108 & 2988 & 858683 \\
\hline Thai registries & 13738188 & $20 \cdot 3 \%$ & 3344 & 5321 & 13801 & 6679 & 30814 & 3284 & 28865 & 695 & 159528 \\
\hline Turkish registries & 18176484 & $23 \cdot 4 \%$ & 3541 & 19782 & 18542 & 9769 & 4480 & 7526 & 57770 & 2856 & 237137 \\
\hline
\end{tabular}


Survival was highest in Japan (36.0\%) and Korea $(31.3 \%)$, and less than $30 \%$ in all other countries (table 6 ; appendix p 210). Survival was in the range $20-30 \%$ in ten countries: Puerto Rico; the USA; China and Israel; five European countries (Ireland [northern Europe]; and
Belgium, Germany, the Netherlands, and Switzerland [western Europe]); and Australia.

Survival trends from 2000 to 2014 increased by 4-5\% in three European countries (Denmark and the UK [northern Europe]; and Germany [western Europe]), and

\begin{tabular}{|c|c|c|c|c|c|c|c|c|c|c|c|}
\hline & \multirow{2}{*}{$\begin{array}{l}\text { Population } \\
\text { covered }\end{array}$} & \multirow{2}{*}{$\begin{array}{l}\text { Percentage } \\
\text { of national } \\
\text { population } \\
\text { covered }\end{array}$} & \multicolumn{8}{|c|}{ Number of patients } & \multirow{2}{*}{$\begin{array}{l}\text { Total } \\
\text { number of } \\
\text { patients } \dagger\end{array}$} \\
\hline & & & Oesophagus & Stomach & Colon & Rectum & Liver & Pancreas & Lung & $\begin{array}{l}\text { Melanoma } \\
\text { of the skin }\end{array}$ & \\
\hline \multicolumn{12}{|c|}{ (Continued from previous page) } \\
\hline Europe & 321365615 & $50.0 \%$ & 294606 & 668096 & 1764170 & 801387 & 283720 & 506723 & 2317434 & 647507 & 14991316 \\
\hline Austria* & 8516916 & $100 \cdot 0 \%$ & 5324 & 19308 & 46127 & 23360 & 10570 & 18371 & 56130 & 19150 & 412683 \\
\hline Belarus (childhood) $\ddagger$ & 1498163 & $100 \cdot 0 \%$ & .. & .. &.. & .. &. &. & " & .. & 1687 \\
\hline Belgium* & 11226322 & $100 \cdot 0 \%$ & 10191 & 15222 & 63540 & 27614 & 7026 & 15015 & 83551 & 21905 & 544110 \\
\hline Bulgaria* & 7201308 & $100 \cdot 0 \%$ & .. & 21404 & 37854 & 22511 & 5704 &. & 50210 & 5875 & 273868 \\
\hline Croatia* & 4255853 & $100 \cdot 0 \%$ & 3007 & 14589 & 27382 & 15309 & 5456 & 8596 & 41744 & 7848 & 222776 \\
\hline Czech Republic* & 10542666 & $100 \cdot 0 \%$ & 7764 & 24157 & 83481 & 34838 & 12056 & 27622 & 93241 & 27800 & 572368 \\
\hline Denmark* & 5646899 & $100 \cdot 0 \%$ & 6556 & 8022 & 40495 & 22384 & 4708 & 13279 & 62402 & 24630 & 365105 \\
\hline Estonia* & 1316203 & $100 \cdot 0 \%$ & 786 & 5212 & 6523 & 3062 & 903 & 2698 & 9201 & 1983 & 59848 \\
\hline Finland ${ }^{*}$ & 5479660 & $100 \cdot 0 \%$ & 3686 & 9871 & 25374 & 12847 & 5096 & 12851 & 31950 & 14949 & 294268 \\
\hline French registriesł & 13891552 & $21 \cdot 7 \%$ & 9958 & 13770 & 53149 & 20018 & 14818 & 15328 & 51989 & 13677 & 460927 \\
\hline German registries & 29655933 & $36 \cdot 8 \%$ & 27208 & 75378 & 191396 & 99791 & 28301 & 57498 & 212897 & 78713 & 1621312 \\
\hline Gibraltar* & 31997 & $100 \cdot 0 \%$ & 12 & 29 & 81 & 21 & 5 & 13 & 63 & 31 & 533 \\
\hline Greece (childhood) $\ddagger$ & 1610335 & $100 \cdot 0 \%$ & .. &. & .. & .. & .. & .. & & .. & 1726 \\
\hline Iceland* & 327318 & $100 \cdot 0 \%$ & 271 & 473 & 1433 & 580 & 165 & 481 & 2314 & 713 & 14782 \\
\hline Ireland* & 4675164 & $100 \cdot 0 \%$ & 4899 & 6801 & 21724 & 9085 & 2178 & 5931 & 26838 & 9470 & 196331 \\
\hline Italian registries & 34479972 & $57.7 \%$ & 12219 & 80686 & 188983 & 53226 & 63084 & 56698 & 203548 & 46607 & 1400117 \\
\hline Latvia* & 1989354 & $100 \cdot 0 \%$ & 1294 & 6878 & 7658 & 4748 & 464 & 1072 & 9691 & 2503 & 71082 \\
\hline Lithuania* & 2916798 & $100 \cdot 0 \%$ & 2130 & 11840 & 11148 & 7694 & 1776 & 5421 & 18499 & 3317 & 139475 \\
\hline Malta* & 417723 & $100 \cdot 0 \%$ & 184 & 656 & 1908 & 781 & 152 & 709 & 2043 & 541 & 15518 \\
\hline Netherlands* & 16868020 & $100 \cdot 0 \%$ & 25342 & 29585 & 126237 & 51839 & 6397 & 28717 & 159895 & 59088 & 987029 \\
\hline Norway* & 5147970 & $100 \cdot 0 \%$ & 3021 & 7548 & 36646 & 16306 & 1916 & 6671 & 33558 & 19994 & 277991 \\
\hline Poland ${ }^{*}$ & 38483957 & $100 \cdot 0 \%$ & 18959 & 79466 & 140075 & 83669 & 20764 & 47635 & 306136 & 35834 & 1344837 \\
\hline Portugal ${ }^{*}$ & 10566132 & $100 \cdot 0 \%$ & 6122 & 33865 & 57219 & 25989 & 5107 & 8303 & 40422 & 9358 & 386853 \\
\hline Romania (Cluj) & 983525 & $5.0 \%$ & 216 & 1201 & 1552 & 657 & 547 & 563 & 2126 & 436 & 14060 \\
\hline Russian registries & 8081400 & $5.6 \%$ & 6000 & 31711 & 28946 & 16305 & 3757 & 10048 & 42434 & 4914 & 245591 \\
\hline Slovakia* & 5422861 & $100 \cdot 0 \%$ & 2794 & 9604 & 23694 & 11066 & 2741 & 6624 & 22971 & 6389 & 156122 \\
\hline Slovenia* & 2066068 & $100 \cdot 0 \%$ & 1202 & 6443 & 12376 & 6682 & 1964 & 4073 & 16051 & 5603 & 102970 \\
\hline Spanish registries & 9396745 & $20 \cdot 3 \%$ & 5637 & 17844 & 54250 & 18245 & 11848 & 12438 & 54237 & 11028 & 376759 \\
\hline Sweden* & 9703247 & $100 \cdot 0 \%$ & 6233 & 13463 & 55664 & 29777 & 7242 & 14240 & 51122 & 36921 & 544531 \\
\hline Swiss registriesł & 4368854 & $53 \cdot 2 \%$ & 3583 & 6135 & 21137 & 8633 & 5070 & 7694 & 27116 & 14893 & 212695 \\
\hline $\mathrm{UK}^{*}$ & 64596700 & $100 \cdot 0 \%$ & 120008 & 116935 & 398118 & 174350 & 53905 & 118134 & 605055 & 163337 & 3673362 \\
\hline Oceania & 27952971 & $100 \cdot 0 \%$ & 21728 & 33411 & 174823 & 67496 & 20311 & 39014 & 162266 & 187512 & 1483573 \\
\hline Australia* & 23457489 & $100 \cdot 0 \%$ & 17877 & 27952 & 144382 & 56260 & 17281 & 33319 & 136318 & 156302 & 1244350 \\
\hline New Zealand ${ }^{*}$ & 4495482 & $100 \cdot 0 \%$ & 3851 & 5459 & 30441 & 11236 & 3030 & 5695 & 25948 & 31210 & 239223 \\
\hline Total & 988680261 & $20 \cdot 2 \%$ & 734428 & 2019382 & 4198637 & 1720488 & 1178364 & 1229379 & 6051262 & 1553109 & 37513025 \\
\hline
\end{tabular}

Table 4: Population covered by participating registries (number of people and percentage of national population) and number of adults diagnosed with cancer of the oesophagus, stomach, colon, rectum, liver, pancreas, or lung, or melanoma of the skin during 2000-14 by continent and country 
the USA (appendix p 229). Survival increased by $6-10 \%$ in Puerto Rico; China, Israel, Japan, and Singapore; six European countries (Ireland and Norway [northern Europe]; Portugal [southern Europe]; Belgium, the Netherlands, and Switzerland [western Europe]); and Australia. The increase in Korea was $12 \cdot 7 \%$.

\section{Stomach}

Results are available for 2019382 adults from 294 registries in 62 countries (table 2, table 4). Age-standardised 5-year net survival was generally in the range $20-40 \%$, with very wide variation in Asia (appendix p 249). Most estimates were considered reliable (table 6; appendix pp 138-151).
In 2010-14, survival was very high in Korea (68.9\%) and Japan (60 -3\%; table 6; appendix p 211). Survival was in the range $30-40 \%$ in 16 countries: Canada and the USA; Puerto Rico and Martinique; five Asian countries (Malaysia [Penang] and Singapore [south Asia]; China and Taiwan [east Asia]; and Israel [west Asia]); six European countries (Italy and Portugal [southern Europe]; and Austria, Belgium, Germany, and Switzerland [western Europe]); and Australia (table 6; appendix p 211).

Survival was in the range $20-29 \%$ in 24 countries (Mauritius, Kuwait, Turkey, 20 European countries, and New Zealand), and less than 20\% in Chile, Ecuador, India (Karunagappally), Thailand, and Bulgaria.

\begin{tabular}{|c|c|c|c|c|c|c|c|c|c|c|c|c|c|}
\hline & \multirow{2}{*}{$\begin{array}{l}\text { Population } \\
\text { covered }\end{array}$} & \multirow{2}{*}{$\begin{array}{l}\text { Percentage } \\
\text { of national } \\
\text { population } \\
\text { covered }\end{array}$} & \multicolumn{10}{|c|}{ Number of patients } & \multirow{2}{*}{$\begin{array}{l}\text { Total } \\
\text { number of } \\
\text { patients } \dagger\end{array}$} \\
\hline & & & $\begin{array}{l}\text { Breast } \\
\text { (women) }\end{array}$ & Cervix & Ovary & Prostate & $\begin{array}{l}\text { Brain } \\
\text { (adults) }\end{array}$ & $\begin{array}{l}\text { Myeloid } \\
\text { (adults) }\end{array}$ & $\begin{array}{l}\text { Lymphoid } \\
\text { (adults) }\end{array}$ & $\begin{array}{l}\text { Brain } \\
\text { (children) }\end{array}$ & $\begin{array}{l}\text { Acute } \\
\text { lympho- } \\
\text { blastic } \\
\text { leukaemia } \\
\text { (children) }\end{array}$ & $\begin{array}{l}\text { Lymph- } \\
\text { oma } \\
\text { (children) }\end{array}$ & \\
\hline Africa & 10533762 & $3 \cdot 5 \%$ & 15117 & 5017 & 1010 & 2726 & 592 & 425 & 2042 & 179 & 64 & 228 & 40197 \\
\hline $\begin{array}{l}\text { Algerian } \\
\text { registries }\end{array}$ & 2447075 & $6 \cdot 3 \%$ & 5196 & 885 & 423 & 764 & 392 & 290 & 1376 & 109 & 45 & 115 & 15602 \\
\hline $\begin{array}{l}\text { Mali } \\
\text { (Bamako) }\end{array}$ & 764245 & $9.0 \%$ & 60 & .. & .. & .. & .. & .. & .. & .. & .. & .. & 60 \\
\hline Mauritius* & 1268567 & $100 \cdot 0 \%$ & 483 & 436 & 244 & 628 & 36 & 42 & 86 & 3 & .. & 5 & 3959 \\
\hline $\begin{array}{l}\text { Morocco } \\
\text { (Casablanca) }\end{array}$ & 2178083 & $12 \cdot 7 \%$ & 4683 & .. & .. & .. &.. & .. & .. & .. & .. & .. & 4683 \\
\hline $\begin{array}{l}\text { Nigeria } \\
\text { (Ibadan) }\end{array}$ & 2797220 & $1.6 \%$ & 3962 & 1578 & 225 & 833 & 148 & 76 & 412 & 45 & 12 & 85 & 8274 \\
\hline $\begin{array}{l}\text { South Africa } \\
\text { (Eastern } \\
\text { Cape) }\end{array}$ & 1078572 & $2.0 \%$ & 733 & 2118 & 118 & 501 & 16 & 17 & 168 & 22 & 7 & 23 & 7619 \\
\hline $\begin{array}{l}\text { America } \\
\text { (Central and } \\
\text { South) }\end{array}$ & 99818363 & $23 \cdot 7 \%$ & 159976 & 49067 & 16023 & 115102 & 8547 & 10842 & 47740 & 4936 & 13299 & 5065 & 700946 \\
\hline $\begin{array}{l}\text { Argentinian } \\
\text { registries } \ddagger\end{array}$ & 3973922 & $9 \cdot 2 \%$ & 15282 & 2467 & 1688 & 7115 & 1217 & 1588 & 4281 & 2662 & 5119 & 1955 & 64151 \\
\hline $\begin{array}{l}\text { Brazilian } \\
\text { registries }\end{array}$ & 15882624 & $7 \cdot 7 \%$ & 49811 & 3083 & 1201 & 7556 & 1011 & 852 & 3270 & 168 & 233 & 143 & 134597 \\
\hline $\begin{array}{l}\text { Chilean } \\
\text { registries }\end{array}$ & 2459133 & $13 \cdot 8 \%$ & 3717 & 1564 & 698 & 4816 & 475 & 652 & 1968 & 83 & 171 & 67 & 26363 \\
\hline $\begin{array}{l}\text { Colombian } \\
\text { registries }\end{array}$ & 4277369 & $9.0 \%$ & 9609 & 5124 & 1759 & 8722 & 1601 & 1476 & 5004 & 243 & 306 & 137 & 56245 \\
\hline Costa Rica* & 4757606 & $100 \cdot 0 \%$ & 12019 & 7466 & 1408 & 11345 & 1067 & 857 & 4646 & 153 & 456 & 103 & 62536 \\
\hline Cuba* & 11379111 & $100 \cdot 0 \%$ & 33313 & 16396 & 4560 & 21358 &.. & .. & 8451 & .. & .. & 563 & 125696 \\
\hline $\begin{array}{l}\text { Ecuadorian } \\
\text { registries }\end{array}$ & 6398546 & $40 \cdot 2 \%$ & 8283 & 5453 & 1732 & 7939 & 1481 & 1489 & 6330 & 324 & 859 & 340 & 53043 \\
\hline Guadeloupe* & 450273 & $100 \cdot 0 \%$ & 1266 & 160 & 110 & 3389 & 55 & 115 & 480 & 14 & 9 & 6 & 7787 \\
\hline Martinique ${ }^{*}$ & 396425 & $100 \cdot 0 \%$ & 2279 & 399 & 191 & 6480 & 182 & 482 & 1257 & 20 & 29 & 10 & 15779 \\
\hline $\begin{array}{l}\text { Mexico } \\
\text { (childhood) } \ddagger\end{array}$ & 35188624 & $100 \cdot 0 \%$ & .. & .. & .. & .. & .. & .. & .. & 1047 & 5647 & 1500 & 8194 \\
\hline Peru (Lima) & 7548697 & $24 \cdot 4 \%$ & 5590 & 2917 & .. & .. & .. & 891 & 3653 & .. & 268 & 112 & 17226 \\
\hline Puerto Rico* & 3686517 & $100 \cdot 0 \%$ & 18807 & 2458 & 1728 & 29855 & 1458 & 2440 & 8400 & 222 & 202 & 129 & 101613 \\
\hline \multirow[t]{2}{*}{ Uruguay* } & 3419516 & $100 \cdot 0 \%$ & .. & 1580 & 948 & 6527 & .. & .. & .. & .. & .. & .. & 27716 \\
\hline & & & & & & & & & & & \multicolumn{3}{|c|}{ (Table 5 continues on next page } \\
\hline
\end{tabular}




\begin{tabular}{|c|c|c|c|c|c|c|c|c|c|c|c|c|c|}
\hline & \multirow{2}{*}{$\begin{array}{l}\text { Population } \\
\text { covered }\end{array}$} & \multirow{2}{*}{$\begin{array}{l}\text { Percentage } \\
\text { of national } \\
\text { population } \\
\text { covered }\end{array}$} & \multicolumn{10}{|c|}{ Number of patients } & \multirow{2}{*}{$\begin{array}{l}\text { Total } \\
\text { number of } \\
\text { patients }\end{array}$} \\
\hline & & & $\begin{array}{l}\text { Breast } \\
\text { (women) }\end{array}$ & Cervix & Ovary & Prostate & $\begin{array}{l}\text { Brain } \\
\text { (adults) }\end{array}$ & $\begin{array}{l}\text { Myeloid } \\
\text { (adults) }\end{array}$ & $\begin{array}{l}\text { Lymphoid } \\
\text { (adults) }\end{array}$ & $\begin{array}{l}\text { Brain } \\
\text { (children) }\end{array}$ & $\begin{array}{l}\text { Acute } \\
\text { lympho- } \\
\text { blastic } \\
\text { leukaemia } \\
\text { (children) }\end{array}$ & $\begin{array}{l}\text { Lymph- } \\
\text { oma } \\
\text { (children) }\end{array}$ & \\
\hline \multicolumn{14}{|c|}{ (Continued from previous page) } \\
\hline $\begin{array}{l}\text { America } \\
\text { (North) }\end{array}$ & 301237785 & $84 \cdot 8 \%$ & 2587798 & 163517 & 312954 & 2703952 & 251888 & 508562 & 1356829 & 27157 & 29995 & 13440 & 14320034 \\
\hline $\begin{array}{l}\text { Canadian } \\
\text { registries }\end{array}$ & 27213277 & $76 \cdot 5 \%$ & 237321 & 16054 & 31395 & 256736 & 28186 & 49474 & 139370 & 2196 & 2712 & 1139 & 1409413 \\
\hline US registries & 274024508 & $85 \cdot 8 \%$ & 2350477 & 147463 & 281559 & 2447216 & 223702 & 459088 & 1217459 & 24961 & 27283 & 12301 & 12910621 \\
\hline Asia & 227771765 & $7 \cdot 2 \%$ & 726968 & 161620 & 109998 & 397673 & 73306 & 140066 & 293307 & 8513 & 11371 & 6380 & 5976959 \\
\hline $\begin{array}{l}\text { Chinese } \\
\text { registries }\end{array}$ & 31755347 & $2 \cdot 3 \%$ & 53791 & 13131 & 10517 & 12380 & 11341 & 12171 & 19388 & 526 & 498 & 213 & 594533 \\
\hline Cyprus* & 1153658 & $100 \cdot 0 \%$ & 5069 & 321 & 553 & 4088 & 394 & 555 & 2326 & 14 & 56 & 39 & 21610 \\
\hline Hong Kong* & 7241700 & $100 \cdot 0 \%$ & 28956 & 4112 & .. &.. & .. &.. &.. & .. & .. & .. & 74721 \\
\hline $\begin{array}{l}\text { Indian } \\
\text { registries }\end{array}$ & 1005294 & $0.1 \%$ & 812 & 753 & 172 & 183 & 162 & 153 & 272 & 18 & 32 & 12 & 4774 \\
\hline $\begin{array}{l}\text { Iran } \\
\text { (Golestan) }\end{array}$ & 1893646 & $2 \cdot 4 \%$ & .. &.. & .. &.. & .. &.. &.. & .. & .. & .. & 1039 \\
\hline |srael* & 7939483 & $100 \cdot 0 \%$ & 51125 & 2942 & 5663 & 32503 & 5235 & 8375 & 30911 & 843 & 754 & 758 & 241881 \\
\hline $\begin{array}{l}\text { Japanese } \\
\text { registries }\end{array}$ & 51445407 & $40 \cdot 6 \%$ & 184372 & 30606 & 31244 & 168505 & 15007 & 37845 & 85640 & 1293 & 1438 & 508 & 1834894 \\
\hline Jordan* & 7416083 & $100 \cdot 0 \%$ & 11584 & 579 & .. & 2457 & 1483 & 1917 & 6511 & 489 & 681 & 480 & 41433 \\
\hline Korea* & 50074400 & $100 \cdot 0 \%$ & 179520 & 58663 & 28076 & 83892 & 17701 & 42322 & 70594 & 2333 & 3389 & 2049 & 1760804 \\
\hline Kuwait* & 3753121 & $100 \cdot 0 \%$ & 2568 & 163 & 221 & 509 & 230 & 346 & 1405 & 49 & 251 & 129 & 8484 \\
\hline $\begin{array}{l}\text { Malaysia } \\
\text { (Penang) }\end{array}$ & 1543500 & $5 \cdot 2 \%$ & 4606 & 1046 & 805 & 915 & 289 & 504 & 1244 & 84 & 156 & 65 & 18805 \\
\hline Mongolia* & 1468823 & $100 \cdot 0 \%$ & 999 &.. & .. &.. &.. &.. &.. & .. &.. &.. & 999 \\
\hline Qatar* & 2172065 & $100 \cdot 0 \%$ & 1861 & 196 & 214 & 586 & 287 & 489 & 1042 & 33 & 71 & 71 & 7642 \\
\hline Singapore* & 3870700 & $100 \cdot 0 \%$ & 22473 & 2943 & 3514 & 7991 & 1202 & 3339 & 7187 & 196 & 388 & 167 & 109992 \\
\hline Taiwan* & 23123866 & $100 \cdot 0 \%$ & 116929 & 29214 & 16872 & 52681 & 8410 & 17813 & 39704 & 1211 & 1811 & 832 & 858683 \\
\hline $\begin{array}{l}\text { Thai } \\
\text { registries }\end{array}$ & 13738188 & $20 \cdot 3 \%$ & 25001 & 12737 & 5469 & 5869 & 2779 & 5177 & 8486 & 385 & 605 & 217 & 159528 \\
\hline $\begin{array}{l}\text { Turkish } \\
\text { registries }\end{array}$ & 18176484 & $23 \cdot 4 \%$ & 37302 & 4214 & 6678 & 25114 & 8786 & 9060 & 18597 & 1039 & 1241 & 840 & 237137 \\
\hline Europe & 321365615 & $50 \cdot 0 \%$ & 2700348 & 267986 & 399675 & 2355249 & 297032 & 436684 & 1182009 & 24316 & 29544 & 14830 & 14991316 \\
\hline Austria* & 8516916 & $100 \cdot 0 \%$ & 74818 & 6455 & 11567 & 75082 & 7615 & 7223 & 31583 &.. & .. & .. & 412683 \\
\hline $\begin{array}{l}\text { Belarus } \\
\text { (childhood) } \ddagger\end{array}$ & 1498163 & $100 \cdot 0 \%$ & .. & .. & .. &.. & .. &.. & .. & 580 & 740 & 367 & 1687 \\
\hline Belgium* & 11226322 & $100 \cdot 0 \%$ & 111685 & 6929 & 10447 & 97316 & 9057 & 19790 & 42772 & 781 & 720 & 549 & 544110 \\
\hline Bulgaria* & 7201308 & $100 \cdot 0 \%$ & 53605 & 16329 & 12206 & 26190 & .. & 6226 & 14919 & .. & 537 & 298 & 273868 \\
\hline Croatia* & 4255853 & $100 \cdot 0 \%$ & 35323 & 5279 & 7138 & 22066 & 7515 & 5026 & 15421 & 403 & 443 & 231 & 222776 \\
\hline $\begin{array}{l}\text { Czech } \\
\text { Republic*}\end{array}$ & 10542666 & $100 \cdot 0 \%$ & 89989 & 14950 & 18875 & 78581 & 11007 & 9734 & 36974 & 489 & 531 & 279 & 572368 \\
\hline Denmark* & 5646899 & $100 \cdot 0 \%$ & 65840 & 5755 & 9024 & 55052 & 8951 & 8951 & 27756 & 493 & 574 & 233 & 365105 \\
\hline Estonia* & 1316203 & $100 \cdot 0 \%$ & 8149 & 2232 & 2122 & 9734 & 1295 & 1512 & 4193 & 103 & 93 & 47 & 59848 \\
\hline Finland* & 5479660 & $100 \cdot 0 \%$ & 62282 & 2318 & 8101 & 66706 & 5953 & 5573 & 25395 & 503 & 572 & 241 & 294268 \\
\hline $\begin{array}{l}\text { French } \\
\text { registriesł }\end{array}$ & 13891552 & $21.7 \%$ & 82538 & 5125 & 8658 & 91806 & 7532 & 18897 & 41784 & 4477 & 4830 & 2573 & 460927 \\
\hline $\begin{array}{l}\text { German } \\
\text { registries }\end{array}$ & 29655933 & $36 \cdot 8 \%$ & 300626 & 24302 & 38064 & 284771 & 27683 & 45934 & 126594 & 691 & 1019 & 446 & 1621312 \\
\hline Gibraltar* & 31997 & $100 \cdot 0 \%$ & 169 & 10 & 14 & 62 & 11 & 4 & 8 & .. &.. & .. & 533 \\
\hline & & & & & & & & & & & & continu & on next page) \\
\hline
\end{tabular}




\begin{tabular}{|c|c|c|c|c|c|c|c|c|c|c|c|c|c|}
\hline & \multirow{2}{*}{$\begin{array}{l}\text { Population } \\
\text { covered }\end{array}$} & \multirow{2}{*}{$\begin{array}{l}\text { Percentage } \\
\text { of national } \\
\text { population } \\
\text { covered }\end{array}$} & \multicolumn{10}{|c|}{ Number of patients } & \multirow{2}{*}{$\begin{array}{l}\text { Total } \\
\text { number of } \\
\text { patients } \dagger\end{array}$} \\
\hline & & & $\begin{array}{l}\text { Breast } \\
\text { (women) }\end{array}$ & Cervix & Ovary & Prostate & $\begin{array}{l}\text { Brain } \\
\text { (adults) }\end{array}$ & $\begin{array}{l}\text { Myeloid } \\
\text { (adults) }\end{array}$ & $\begin{array}{l}\text { Lymphoid } \\
\text { (adults) }\end{array}$ & $\begin{array}{l}\text { Brain } \\
\text { (children) }\end{array}$ & $\begin{array}{l}\text { Acute } \\
\text { lympho- } \\
\text { blastic } \\
\text { leukaemia } \\
\text { (children) }\end{array}$ & $\begin{array}{l}\text { Lymph- } \\
\text { oma } \\
\text { (children) }\end{array}$ & \\
\hline \multicolumn{14}{|c|}{ (Continued from previous page) } \\
\hline $\begin{array}{l}\text { Greece } \\
\text { (childhood)‡ }\end{array}$ & 1610335 & $100 \cdot 0 \%$ & &.$\cdot$ & .. &.. &. & .. & .. & 237 & 1092 & 397 & 1726 \\
\hline Iceland* & 327318 & $100 \cdot 0 \%$ & 2743 & 225 & 276 & 3083 & 348 & 433 & 1167 & 35 & 31 & 11 & 14782 \\
\hline Ireland* & 4675164 & $100 \cdot 0 \%$ & 34632 & 3573 & 4952 & 37536 & 4605 & 6250 & 16806 & 396 & 461 & 194 & 196331 \\
\hline $\begin{array}{l}\text { Italian } \\
\text { registries }\end{array}$ & 34479972 & $57 \cdot 7 \%$ & 250204 & 13394 & 31025 & 196256 & 28325 & 49653 & 121301 & 1613 & 2022 & 1273 & 1400117 \\
\hline Latvia* & 1989354 & $100 \cdot 0 \%$ & 13020 & 3148 & 3842 & 10674 & 1415 & 1749 & 2679 & 100 & 147 & .. & 71082 \\
\hline Lithuania* & 2916798 & $100 \cdot 0 \%$ & 17699 & 6318 & 5452 & 30156 & 2942 & 4850 & 9741 & 136 & 250 & 106 & 139475 \\
\hline Malta* & 417723 & $100 \cdot 0 \%$ & 3523 & 136 & 547 & 2069 & 315 & 492 & 1383 & 24 & 35 & 20 & 15518 \\
\hline Netherlands* & 16868020 & $100 \cdot 0 \%$ & 198074 & 10317 & 19252 & 142578 & 17261 & 30256 & 78420 & 1428 & 1588 & 755 & 987029 \\
\hline Norway* & 5147970 & $100 \cdot 0 \%$ & 43349 & 4458 & 7207 & 57657 & 5647 & 8374 & 24389 & 484 & 511 & 255 & 277991 \\
\hline Poland ${ }^{*}$ & 38483957 & $100 \cdot 0 \%$ & 220036 & 48857 & 53462 & 131099 & 37794 & 21008 & 94159 & 2071 & 2505 & 1308 & 1344837 \\
\hline Portugal* & 10566132 & $100 \cdot 0 \%$ & 69599 & 9013 & 6532 & 64886 & 7348 & 9414 & 32024 & 549 & 627 & 476 & 386853 \\
\hline $\begin{array}{l}\text { Romania } \\
\text { (Cluj) }\end{array}$ & 983525 & $5.0 \%$ & 2205 & 1004 & 460 & 1301 & 291 & 482 & 969 & 19 & 21 & 10 & 14060 \\
\hline $\begin{array}{l}\text { Russian } \\
\text { registries }\end{array}$ & 8081400 & $5 \cdot 6 \%$ & 41903 & 10897 & 10628 & 20346 & 4449 & 3190 & 9209 & 327 & 320 & 207 & 245591 \\
\hline Slovakia* & 5422861 & $100 \cdot 0 \%$ & 23698 & 6170 & 5207 & 14376 & 3695 & 4466 & 11821 & 323 & 304 & 179 & 156122 \\
\hline Slovenia* & 2066068 & $100 \cdot 0 \%$ & 15822 & 2281 & 2750 & 14932 & 1943 & 3026 & 7498 & 114 & 122 & 88 & 102970 \\
\hline $\begin{array}{l}\text { Spanish } \\
\text { registries }\end{array}$ & 9396745 & $20 \cdot 3 \%$ & 56759 & 5023 & 7710 & 63237 & 8685 & 13483 & 31782 & 1521 & 1917 & 1115 & 376759 \\
\hline Sweden* & 9703247 & $100 \cdot 0 \%$ & 102483 & 6816 & 12132 & 139051 & 9327 & 14280 & 43784 & 761 & 926 & 309 & 544531 \\
\hline $\begin{array}{l}\text { Swiss } \\
\text { registries }\end{array}$ & 4368854 & $53 \cdot 2 \%$ & 39262 & 1858 & 4964 & 40528 & 4102 & 7175 & 18923 & 565 & 733 & 324 & 212695 \\
\hline $\mathrm{UK}^{*}$ & 64596700 & $100 \cdot 0 \%$ & 680313 & 44814 & 97061 & 578118 & 71921 & 129233 & 308555 & 5093 & 5873 & 2539 & 3673362 \\
\hline Oceania & 27952971 & $100 \cdot 0 \%$ & 232346 & 13537 & 25841 & 290176 & 25294 & 54647 & 129127 & 1713 & 3078 & 1253 & 1483573 \\
\hline Australia* & 23457489 & $100 \cdot 0 \%$ & 193134 & 11065 & 21124 & 247000 & 21569 & 46955 & 108727 & 1484 & 2565 & 1036 & 1244350 \\
\hline $\begin{array}{l}\text { New } \\
\text { Zealand* }\end{array}$ & 4495482 & $100 \cdot 0 \%$ & 39212 & 2472 & 4717 & 43176 & 3725 & 7692 & 20400 & 229 & 513 & 217 & 239223 \\
\hline Total & 988680261 & $20 \cdot 2 \%$ & 6422553 & 660744 & 865501 & 5864878 & 656659 & 1151226 & 3011054 & 66814 & 87351 & 41196 & 37513025 \\
\hline $\begin{array}{l}\text { Populations given } \\
\text { (2010). Subnation } \\
\text { Mongolia, and Mo } \\
\text { national populatio }\end{array}$ & $\begin{array}{l}\text { populations wer } \\
\text { occo only provide } \\
\text {. Total is for all }\end{array}$ & $\begin{array}{l}\text { ancers (seet } \\
\text { avided by th } \\
\text { ancer an }\end{array}$ & $\begin{array}{l}\text { table 4). } \neq \mathrm{Da} \\
\text { tr women }\end{array}$ & $\begin{array}{l}\text { hed. Bela } \\
\text { a popula } \\
\text { h } 100 \%\end{array}$ & $\begin{array}{l}\text { Greece, } \\
\text { s showr } \\
\text { rage of }\end{array}$ & $\begin{array}{l}\text { or women. Pc } \\
\text { tional popul }\end{array}$ & $\begin{array}{l}\text { opulation sh } \\
\text { ation for chi }\end{array}$ & $\begin{array}{l}\text { childhood } \\
\text { for France } \\
\text { od maligna }\end{array}$ & $\begin{array}{l}\text { ancers: nationa, } \\
\text { excludes Guade } \\
\text { incies only. }\end{array}$ & $\begin{array}{l}\text { Guadeloup } \\
\text { al populatio } \\
\text { loupe and }\end{array}$ & $\begin{array}{l}\text { ong Kong, Pc } \\
\text { shown are fo } \\
\text { rtinique. *Da }\end{array}$ & $\begin{array}{l}\text { d, Portugal, } \\
\text { Idren (0-14 } \\
\text { ith } 100 \% \text { co }\end{array}$ & $\begin{array}{l}\text { and Taiwan } \\
\text { years). Mali, } \\
\text { verage of the }\end{array}$ \\
\hline
\end{tabular}

Survival trends between 1995-99 and 2010-14 were rather flat in most countries (appendix p 230), but after 2000 , survival increased by up to $5 \%$ in six European countries (Denmark, Lithuania, and the UK [northern Europe]; Poland [eastern Europe]; and Austria and the Netherlands [western Europe]). Over the same 20 years, 5 -year survival increased by $6-10 \%$ in Canada, Israel, Japan, Estonia, and Ireland, by $11 \%$ in the USA and Germany, and by $20 \%$ or more in China and Korea.

\section{Colon}

Results are available for 4198637 adults from 296 registries in 65 countries (tables 2, 4). Survival for colon cancer varied widely, especially in Central and South America, Asia, and Europe (figure 4; appendix p 250). Most estimates were considered reliable (table 6; appendix pp 139-151).

For patients diagnosed during 2010-14, survival was higher than $70 \%$ in three countries: Israel, Korea, and Australia. Survival was in the range 60-69\% in 25 countries: 


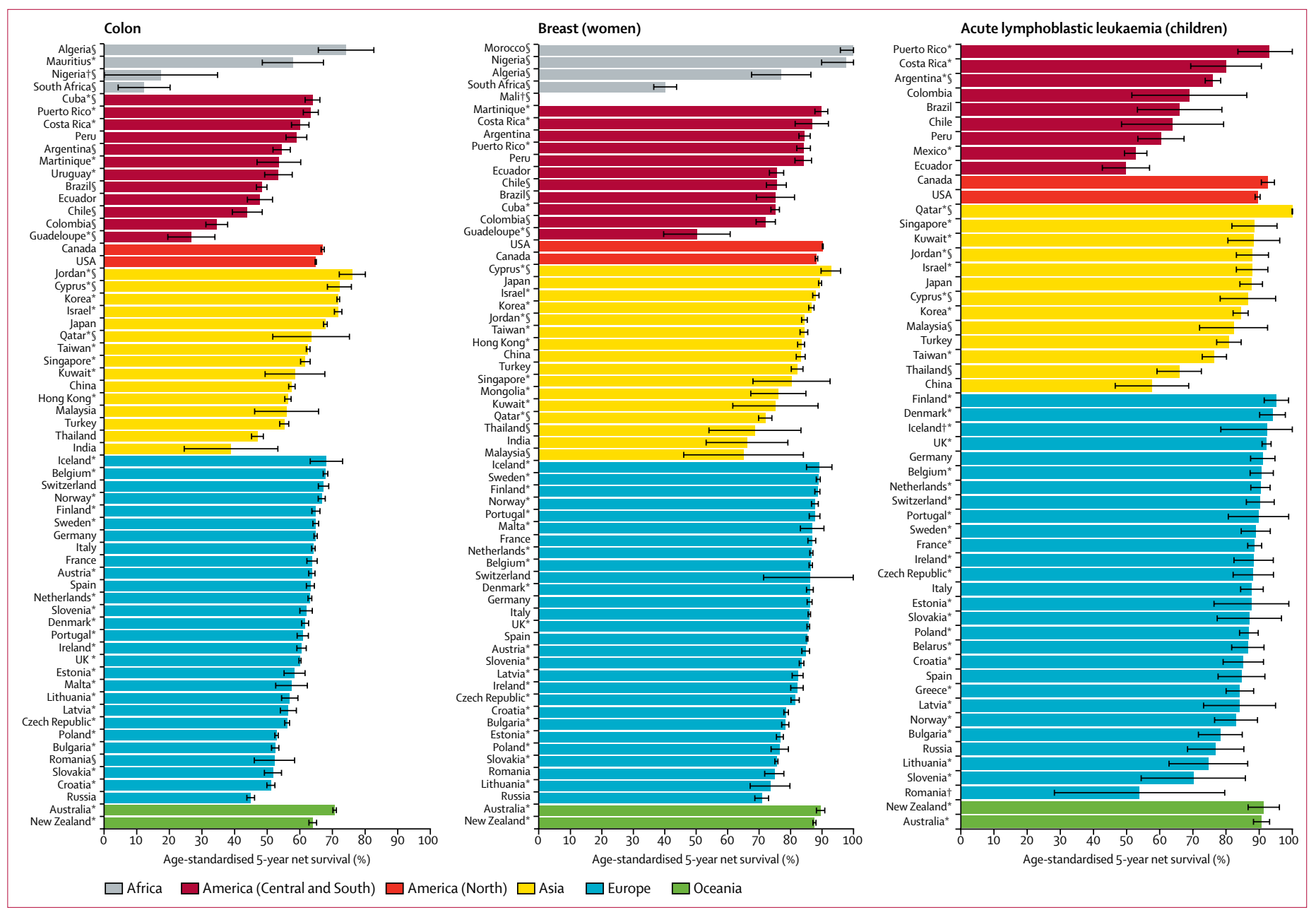

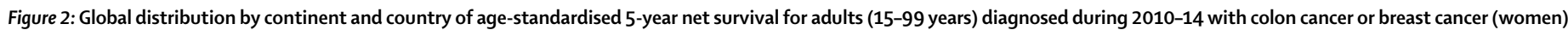
and children (0-14 years) diagnosed with acute lymphoblastic leukaemia

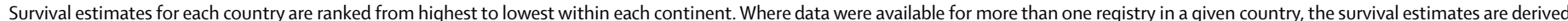

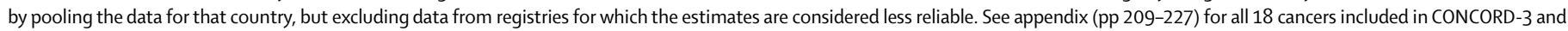

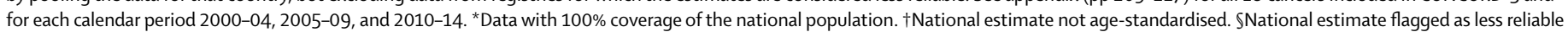
because the only available estimates are from a registry or registries in this category.

Costa Rica and Puerto Rico; Canada and the USA; Japan, Singapore, and Taiwan; 17 European countries (Denmark, Finland, Iceland, Ireland, Norway, Sweden, and the UK [northern Europe]; Italy, Portugal, Slovenia, and Spain [southern Europe]; and Austria, Belgium, France, Germany, the Netherlands, and Switzerland [western Europe]); and New Zealand (table 6; appendix p 212).

Survival ranged from $50 \%$ to $60 \%$ in 18 countries: Mauritius; three Central and South American countries (Martinique, Peru [Lima], and Uruguay); five Asian countries (Malaysia [Penang; south Asia]; China and Hong Kong [east Asia]; and Kuwait and Turkey [west Asia]); and nine European countries (Estonia, Lithuania, and Latvia [northern Europe]; Croatia and Malta [southern Europe]; and Bulgaria, the Czech Republic, Poland, and Slovakia [eastern Europe]). 5-year net survival was less than 50\% in Ecuador, Thailand, Russia, and India (table 6).
Survival trends between 1995-99 and 2000-14 were generally flat or increasing (appendix p 231). 5-year survival increased over this period by $5-10 \%$ in 14 countries: Canada; Japan and Taiwan; ten European countries (Estonia, Finland, Ireland, Lithuania, and Sweden [northern Europe]; Italy and Malta [southern Europe]; Austria, France, and the Netherlands [western Europe]); and Australia. Over the same period, survival increased by more than $10 \%$ in China, Israel, and Korea, and in 13 European countries (Denmark, Iceland, Latvia, Norway, and the UK [northern Europe]; Portugal, Slovenia, and Spain [southern Europe]; and Bulgaria, the Czech Republic, and Poland [eastern Europe]; and Germany and Switzerland [western Europe]).

\section{Rectum}

Results are available for 1720488 adults from 294 registries in 64 countries (table 2, table 4). Similarly 


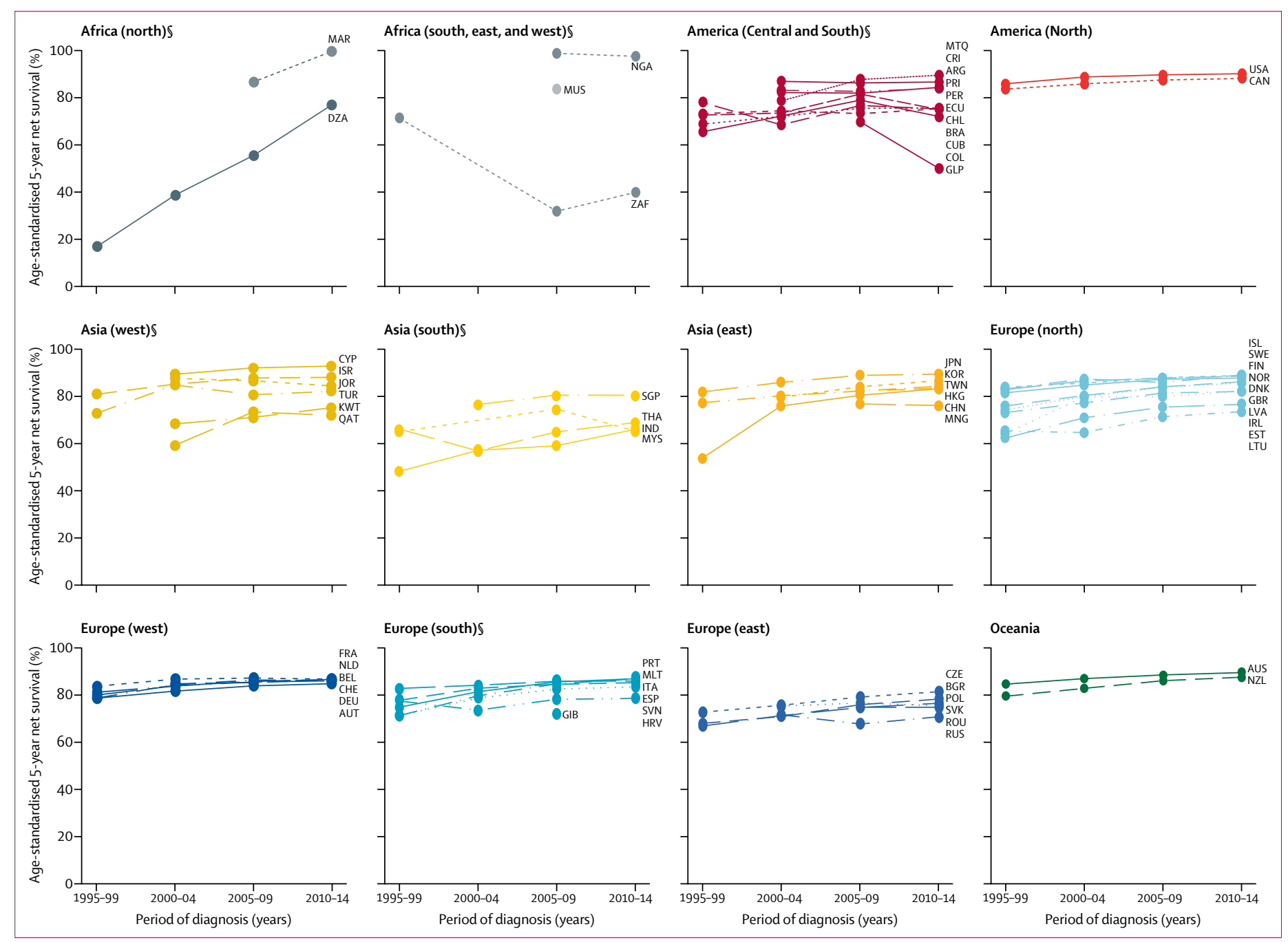

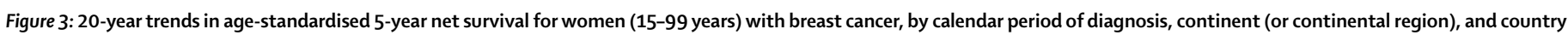

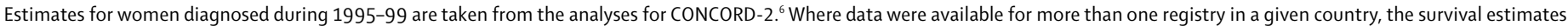
are derived by pooling the data for that country, excluding data from registries for which the survival estimates are considered less reliable. See appendix (pp 227-246) for other cancers. Standard International Organization for Standardization abbreviations for country names: Algeria=DZA; Argentina=ARG; Australia=AUS; Austria=AUT; Belgium=BEL; Brazil=BRA;

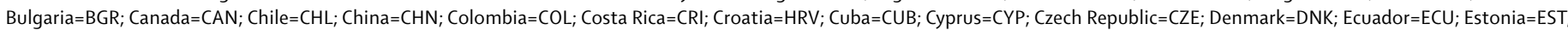

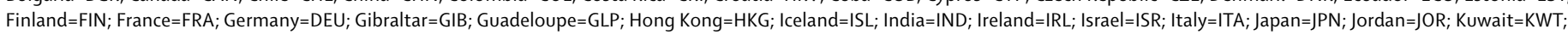
Latvia=LVA; Lithuania=LTU; Malaysia=MYS; Malta=MLT; Martinique=MTQ; Mauritius=MUS; Mongolia=MNG; Morocco=MAR; Netherlands=NLD; New Zealand=NZL; Nigeria=NGA; Norway=NOR; Peru=PER; Poland=POL; Portugal=PRT; Puerto Rico=PRI; Qatar=QAT; Romania=ROU; Russia=RUS; Singapore=SGP; Slovakia=SVK; Slovenia=SVN; South Africa=ZAF; South Korea=KOR; Spain=ESP; Sweden=SWE; Switzerland=CHE; Taiwan=TWN; Thailand=THA; Turkey=TUR; UK=GBR; USA=USA. SContinent or continental region with one or more national estimates flagged as less reliable.

to colon cancer, 5-year net survival for rectal cancer varied widely. The range of survival estimates in Asia for 2010-14 was even wider than that for colon cancer (appendix p 251). Almost all the survival estimates were considered reliable (table 6; appendix pp 139-151).

Survival was higher than $70 \%$ in Korea and Australia (table 6). For patients diagnosed during 2010-14, survival was in the range $60-69 \%$ in 24 countries: Canada and the USA; four Asian countries (Singapore [south Asia]; Japan and Taiwan [east Asia]; and Israel [west Asia]); 17 European countries (Denmark, Finland, Iceland, Ireland, Norway, Sweden, and the UK [northern Europe];
Italy, Portugal, Slovenia, and Spain [southern Europe]; and Austria, Belgium, France, Germany, the Netherlands, and Switzerland [western Europe]); and New Zealand (table 6; appendix p 213).

Survival was in the range $50-59 \%$ in 18 countries: six countries in Central and South America (Argentina, Costa Rica, Martinique, Peru [Lima], Puerto Rico, and Uruguay); five Asian countries (Malaysia [Penang; south Asia]; China and Hong Kong [east Asia]; and Kuwait and Turkey [west Asia]); and six European countries (Estonia, Latvia, and Lithuania [northern Europe]; Malta [southern Europe]; and the Czech Republic and Romania 
[Cluj; eastern Europe]). 5-year survival was less than 50\% in Slovakia, Poland, Croatia, Bulgaria, Ecuador, Thailand, Russia, and India (table 6).
Survival trends between 1995-99 and 2000-14 were generally increasing, especially since 2000, in Asia, Europe, and Oceania (appendix p 232). Survival increased

\begin{tabular}{|c|c|c|c|c|c|c|c|c|}
\hline & \multicolumn{6}{|c|}{ Gastrointestinal cancers } & \multirow[t]{2}{*}{ Lung } & \multirow{2}{*}{$\begin{array}{l}\text { Melanoma of } \\
\text { the skin }\end{array}$} \\
\hline & Oesophagus & Stomach & Colon & Rectum & Liver & Pancreas & & \\
\hline \multicolumn{9}{|l|}{ Africa } \\
\hline \multicolumn{9}{|c|}{ Algeria (three registries) } \\
\hline 2000-04 &.. & $\begin{array}{l}20 \cdot 7 \S \\
(14 \cdot 3-27 \cdot 1)\end{array}$ & $\begin{array}{l}88 \cdot 15 \\
(80 \cdot 1-96 \cdot 0)\end{array}$ & $\begin{array}{l}63 \cdot 45 \\
(48 \cdot 3-78 \cdot 5)\end{array}$ & $\begin{array}{c}6 \cdot 2+5 \\
(0 \cdot 0-13 \cdot 7)\end{array}$ & $\begin{array}{c}8 \cdot 3+5 \\
(0 \cdot 0-20 \cdot 3)\end{array}$ & $\begin{array}{l}18 \cdot 0 \mathrm{~S} \\
(14 \cdot 0-22 \cdot 0)\end{array}$ & $\begin{array}{c}6 \cdot 9+5 \\
(0 \cdot 0-18 \cdot 5)\end{array}$ \\
\hline 2005-09 & $\begin{array}{l}55 \cdot 4+5 \\
(36 \cdot 4-74 \cdot 5)\end{array}$ & $\begin{array}{l}42 \cdot 45 \\
(36 \cdot 4-48 \cdot 4)\end{array}$ & $\begin{array}{l}76 \cdot 7 \S \\
(69 \cdot 6-83 \cdot 9)\end{array}$ & $\begin{array}{l}64 \cdot 5 \S \\
(56 \cdot 4-72 \cdot 7)\end{array}$ & $\begin{array}{l}13 \cdot 9 \$ \\
(8 \cdot 6-19 \cdot 2)\end{array}$ & $\begin{array}{l}30 \cdot 25 \\
(21 \cdot 3-39 \cdot 2)\end{array}$ & $\begin{array}{l}30 \cdot 2 \S \\
(25 \cdot 7-34 \cdot 8)\end{array}$ & $\begin{array}{l}63 \cdot 45 \\
(59 \cdot 4-67 \cdot 3)\end{array}$ \\
\hline 2010-14 & $\begin{array}{l}37 \cdot 35 \\
(23 \cdot 4-51 \cdot 1)\end{array}$ & $\begin{array}{c}41 \cdot 65 \\
(35 \cdot 5-47 \cdot 7)\end{array}$ & $\begin{array}{l}74 \cdot 25 \\
(65 \cdot 7-82 \cdot 7)\end{array}$ & $\begin{array}{l}67 \cdot 35 \\
(58 \cdot 0-76 \cdot 5)\end{array}$ & $\begin{array}{l}13 \cdot 55 \\
(6 \cdot 9-20 \cdot 1)\end{array}$ & $\begin{array}{l}29 \cdot 85 \\
(18 \cdot 7-40 \cdot 9)\end{array}$ & $\begin{array}{l}33 \cdot 7 \S \\
(28 \cdot 5-38 \cdot 9)\end{array}$ & $\begin{array}{l}54 \cdot 95 \\
(47 \cdot 3-62 \cdot 6)\end{array}$ \\
\hline \multicolumn{9}{|c|}{ Mali (Bamako) } \\
\hline 2000-04 &.. &.. &.. &.. & .. &.. & .. &.. \\
\hline $2005-09$ &.. &.. & .. & .. &.. &.. & .. &.. \\
\hline 2010-14 &.. &. &. &. &. &.. & .. &. \\
\hline \multicolumn{9}{|l|}{ Mauritius* } \\
\hline 2000-04 & .. & .. & .. & .. & .. &.. & .. & .. \\
\hline $2005-09$ &.$\cdot$ & $\begin{array}{l}44 \cdot 3 \\
(36 \cdot 8-51 \cdot 7)\end{array}$ & $\begin{array}{l}65 \cdot 9 \\
(56 \cdot 7-75 \cdot 1)\end{array}$ & $\begin{array}{l}83 \cdot 65 \\
(75 \cdot 0-92 \cdot 1)\end{array}$ &. &.. & $\begin{array}{l}31 \cdot 7 \\
(25 \cdot 9-37 \cdot 6)\end{array}$ &. \\
\hline 2010-14 & $\begin{array}{l}28 \cdot 1 \dagger \\
(14 \cdot 6-41 \cdot 5)\end{array}$ & $\begin{array}{l}25 \cdot 7 \\
(18 \cdot 0-33 \cdot 3)\end{array}$ & $\begin{array}{l}57 \cdot 9 \\
(48 \cdot 5-67 \cdot 2)\end{array}$ & $\begin{array}{l}72 \cdot 95 \\
(62 \cdot 7-83 \cdot 0)\end{array}$ & $\begin{array}{c}17 \cdot 0 \dagger \\
(2 \cdot 6-31 \cdot 3)\end{array}$ & $\begin{array}{l}24 \cdot 5 \dagger \\
(11 \cdot 4-37 \cdot 6)\end{array}$ & $\begin{array}{l}20 \cdot 4 \\
(13 \cdot 2-27 \cdot 7)\end{array}$ &.. \\
\hline \multicolumn{9}{|c|}{ Morocco (Casablanca) } \\
\hline 2000-04 &.. &.. & .. & .. &.. &.. & .. & .. \\
\hline $2005-09$ &.. &.. & .. & .. & .. &.. & .. & .. \\
\hline 2010-14 &.. & .. &. &. &. &.. & .. & .. \\
\hline \multicolumn{9}{|c|}{ Nigeria (Ibadan) } \\
\hline 2000-04 & .. & .. & .. & .. & .. & .. & .. & .. \\
\hline 2005-09 &.. & .. & $\begin{array}{l}41 \cdot 2+5 \\
(16 \cdot 9-65 \cdot 6)\end{array}$ & $\begin{array}{c}25 \cdot 9+5 \\
(0 \cdot 0-53 \cdot 7)\end{array}$ & .. &.. & & $\begin{array}{l}100 \cdot 0+5 \\
(100 \cdot 0-100 \cdot 0)\end{array}$ \\
\hline 2010-14 &.. & .. & $\begin{array}{c}17 \cdot 4+5 \\
(0 \cdot 1-34 \cdot 8)\end{array}$ & $\begin{array}{c}16 \cdot 9+5 \\
(0 \cdot 0-37 \cdot 8)\end{array}$ &. & .. & .. & $\begin{array}{l}97 \cdot 7+5 \\
(74 \cdot 6-100 \cdot 0)\end{array}$ \\
\hline \multicolumn{9}{|c|}{ South Africa (Eastern Cape) } \\
\hline 2000-04 & $\begin{array}{c}12 \cdot 1+5 \\
(0 \cdot 0-27 \cdot 0)\end{array}$ &.$\cdot$ & .. &. & $\begin{array}{c}27 \cdot 1+5 \\
(0 \cdot 0-57 \cdot 1)\end{array}$ &.. & .. & .. \\
\hline 2005-09 & $\begin{array}{l}19 \cdot 25 \\
(12 \cdot 0-26 \cdot 4)\end{array}$ & $\begin{array}{l}25 \cdot 0+5 \\
(6 \cdot 1-44 \cdot 0)\end{array}$ & $\begin{array}{c}31 \cdot 9+5 \\
(10 \cdot 7-53 \cdot 1)\end{array}$ & $\begin{array}{c}19 \cdot 9+5 \\
(0 \cdot 0-46 \cdot 4)\end{array}$ & .. &.. & $\begin{array}{c}16 \cdot 7+5 \\
(0.8-32 \cdot 5)\end{array}$ & .. \\
\hline $2010-14$ & $\begin{array}{l}18 \cdot 05 \\
(12 \cdot 6-23 \cdot 4)\end{array}$ & $\begin{array}{c}25 \cdot 6+5 \\
(7 \cdot 2-43 \cdot 9)\end{array}$ & $\begin{array}{c}12 \cdot 35 \\
(4 \cdot 3-20 \cdot 2)\end{array}$ & $\begin{array}{c}9 \cdot 1+5 \\
(0 \cdot 0-21 \cdot 5)\end{array}$ & $\begin{array}{c}0.0+5 \\
(0.0-0.0)\end{array}$ & $\begin{array}{c}21 \cdot 8+5 \\
(0 \cdot 0-49 \cdot 5)\end{array}$ & $\begin{array}{l}15 \cdot 05 \\
(6 \cdot 1-24 \cdot 0)\end{array}$ & $\begin{array}{c}16 \cdot 7+5 \\
(0 \cdot 0-41 \cdot 0)\end{array}$ \\
\hline \multicolumn{9}{|c|}{ America (Central and South) } \\
\hline \multicolumn{9}{|c|}{ Argentina (five registries) $\ddagger$} \\
\hline 2000-04 & $\begin{array}{l}18 \cdot 7 \S \\
(12 \cdot 7-24 \cdot 8)\end{array}$ & $\begin{array}{l}21 \cdot 75 \\
(17 \cdot 1-26 \cdot 2)\end{array}$ & $\begin{array}{l}54 \cdot 2 \Omega \\
(49 \cdot 7-58 \cdot 7)\end{array}$ & $\begin{array}{l}48 \cdot 9 \\
(41 \cdot 5-56 \cdot 3)\end{array}$ & $\begin{array}{l}14 \cdot 15 \\
(6 \cdot 0-22 \cdot 1)\end{array}$ & $\begin{array}{c}9.65 \\
(5 \cdot 5-13 \cdot 6)\end{array}$ & $\begin{array}{l}19 \cdot 55 \\
(15 \cdot 5-23 \cdot 5)\end{array}$ & $\begin{array}{l}68 \cdot 4 \\
(60 \cdot 6-76 \cdot 3)\end{array}$ \\
\hline 2005-09 & $\begin{array}{l}15 \cdot 05 \\
(11 \cdot 4-18 \cdot 6)\end{array}$ & $\begin{array}{l}19 \cdot 3 \$ \\
(16 \cdot 9-21 \cdot 7)\end{array}$ & $\begin{array}{l}51 \cdot 2 \S \\
(49 \cdot 0-53 \cdot 5)\end{array}$ & $\begin{array}{l}47 \cdot 5 \\
(43 \cdot 3-51 \cdot 7)\end{array}$ & $\begin{array}{l}11 \cdot 45 \\
(8 \cdot 2-14 \cdot 5)\end{array}$ & $\begin{array}{c}8 \cdot 35 \\
(6 \cdot 0-10 \cdot 7)\end{array}$ & $\begin{array}{l}12 \cdot 45 \\
(10 \cdot 9-14 \cdot 0)\end{array}$ & $\begin{array}{l}68 \cdot 1 \\
(63 \cdot 4-72 \cdot 9)\end{array}$ \\
\hline 2010-14 & $\begin{array}{l}16 \cdot 45 \\
(11 \cdot 9-21 \cdot 0)\end{array}$ & $\begin{array}{l}21 \cdot 5 \S \\
(18 \cdot 5-24 \cdot 4)\end{array}$ & $\begin{array}{l}54 \cdot 45 \\
(51 \cdot 8-57 \cdot 1)\end{array}$ & $\begin{array}{l}49 \cdot 9 \\
(45 \cdot 3-54 \cdot 4)\end{array}$ & $\begin{array}{l}12 \cdot 6 \S \\
(8 \cdot 7-16 \cdot 6)\end{array}$ & $\begin{array}{l}11 \cdot 4 \S \\
(8 \cdot 1-14 \cdot 6)\end{array}$ & $\begin{array}{l}13 \cdot 15 \\
(11 \cdot 2-15 \cdot 1)\end{array}$ & $\begin{array}{l}71 \cdot 0 \\
(65 \cdot 6-76 \cdot 4)\end{array}$ \\
\hline \multicolumn{9}{|c|}{ Brazil (six registries) } \\
\hline 2000-04 & $\begin{array}{l}10 \cdot 75 \\
(9 \cdot 0-12 \cdot 4)\end{array}$ & $\begin{array}{l}19 \cdot 15 \\
(17 \cdot 9-20 \cdot 3)\end{array}$ & $\begin{array}{l}44 \cdot 55 \\
(42 \cdot 9-46 \cdot 0)\end{array}$ & $\begin{array}{l}37 \cdot 7 \S \\
(35 \cdot 7-39 \cdot 6)\end{array}$ & $\begin{array}{l}15 \cdot 4 \varsigma \\
(10 \cdot 4-20 \cdot 5)\end{array}$ & $\begin{array}{c}3.95 \\
(3 \cdot 0-4 \cdot 9)\end{array}$ & $\begin{array}{l}10 \cdot 7 \\
(8 \cdot 3-13 \cdot 1)\end{array}$ & $\begin{array}{l}76 \cdot 7 \\
(71 \cdot 5-81 \cdot 8)\end{array}$ \\
\hline $2005-09$ & $\begin{array}{l}12 \cdot 5 \S \\
(10 \cdot 6-14 \cdot 5)\end{array}$ & $\begin{array}{l}24 \cdot 7 \S \\
(23 \cdot 2-26 \cdot 2)\end{array}$ & $\begin{array}{l}50 \cdot 65 \\
(49 \cdot 3-52 \cdot 0)\end{array}$ & $\begin{array}{l}45 \cdot 7 \S \\
(43 \cdot 7-47 \cdot 8)\end{array}$ & $\begin{array}{c}9 \cdot 65 \\
(6 \cdot 5-12 \cdot 8)\end{array}$ & $\begin{array}{c}9 \cdot 15 \\
(7 \cdot 3-10 \cdot 9)\end{array}$ & $\begin{array}{c}7 \cdot 8 \\
(5 \cdot 8-9 \cdot 8)\end{array}$ & $\begin{array}{l}75 \cdot 9 \\
(71 \cdot 7-80 \cdot 1)\end{array}$ \\
\hline 2010-14 & $\begin{array}{c}9 \cdot 75 \\
(7 \cdot 9-11 \cdot 4)\end{array}$ & $\begin{array}{l}20 \cdot 65 \\
(18 \cdot 9-22 \cdot 2)\end{array}$ & $\begin{array}{l}48 \cdot 35 \\
(46 \cdot 7-49 \cdot 9)\end{array}$ & $\begin{array}{l}42 \cdot 45 \\
(40 \cdot 1-44 \cdot 6)\end{array}$ & $\begin{array}{l}11 \cdot 25 \\
(7 \cdot 6-14 \cdot 7)\end{array}$ & $\begin{array}{c}9.5 \$ \\
(7 \cdot 4-11 \cdot 5)\end{array}$ & $\begin{array}{c}8.5 \\
(5 \cdot 3-11 \cdot 6)\end{array}$ & $\begin{array}{l}70 \cdot 0 \\
(65 \cdot 4-74 \cdot 7)\end{array}$ \\
\hline
\end{tabular}




\begin{tabular}{|c|c|c|c|c|c|c|c|c|}
\hline & \multicolumn{6}{|c|}{ Gastrointestinal cancers } & \multirow[t]{2}{*}{ Lung } & \multirow{2}{*}{$\begin{array}{l}\text { Melanoma of } \\
\text { the skin }\end{array}$} \\
\hline & Oesophagus & Stomach & Colon & Rectum & Liver & Pancreas & & \\
\hline \multicolumn{9}{|c|}{ (Continued from previous page) } \\
\hline \multicolumn{9}{|c|}{ Chile (four registries) } \\
\hline $2000-04$ & $\begin{array}{c}7 \cdot 0 \\
(3 \cdot 1-10 \cdot 9)\end{array}$ & $\begin{array}{l}14 \cdot 5 \\
(11 \cdot 7-17 \cdot 4)\end{array}$ & $\begin{array}{l}35 \cdot 5 \\
(28 \cdot 6-42 \cdot 3)\end{array}$ & $\begin{array}{l}35 \cdot 9 \\
(26 \cdot 3-45 \cdot 5)\end{array}$ & $\begin{array}{c}3 \cdot 6+5 \\
(0 \cdot 0-7 \cdot 4)\end{array}$ & $\begin{array}{c}2 \cdot 2 \\
(0 \cdot 2-4 \cdot 2)\end{array}$ & $\begin{array}{c}7 \cdot 15 \\
(4 \cdot 4-9 \cdot 8)\end{array}$ & $\begin{array}{l}57 \cdot 4 \\
(46 \cdot 6-68 \cdot 1)\end{array}$ \\
\hline $2005-09$ & $\begin{array}{l}10 \cdot 8 \\
(7 \cdot 5-14 \cdot 1)\end{array}$ & $\begin{array}{l}16 \cdot 3 \\
(14 \cdot 7-18 \cdot 0)\end{array}$ & $\begin{array}{l}47 \cdot 1 \\
(43 \cdot 1-51 \cdot 0)\end{array}$ & $\begin{array}{l}39 \cdot 5 \\
(33 \cdot 9-45 \cdot 0)\end{array}$ & $\begin{array}{c}2 \cdot 75 \\
(1 \cdot 0-4 \cdot 4)\end{array}$ & $\begin{array}{c}3 \cdot 6 \\
(1 \cdot 9-5 \cdot 4)\end{array}$ & $\begin{array}{c}6 \cdot 3 \$ \\
(4 \cdot 7-7 \cdot 8)\end{array}$ & $\begin{array}{l}64 \cdot 4 \\
(54 \cdot 4-74 \cdot 4)\end{array}$ \\
\hline 2010-14 & $\begin{array}{c}8 \cdot 7 \\
(0 \cdot 3-17 \cdot 0)\end{array}$ & $\begin{array}{l}16 \cdot 7 \\
(14 \cdot 2-19 \cdot 3)\end{array}$ & $\begin{array}{l}43 \cdot 95 \\
(39 \cdot 3-48 \cdot 5)\end{array}$ & $\begin{array}{l}32 \cdot 7 \S \\
(26 \cdot 6-38 \cdot 8)\end{array}$ & $\begin{array}{c}3 \cdot 75 \\
(0.7-6 \cdot 8)\end{array}$ & $\begin{array}{c}4 \cdot 4^{\dagger} \\
(1 \cdot 0-7 \cdot 8)\end{array}$ & $\begin{array}{c}4 \cdot 65 \\
(3 \cdot 0-6 \cdot 2)\end{array}$ & $\begin{array}{l}59 \cdot 7 \S \\
(49 \cdot 4-69 \cdot 9)\end{array}$ \\
\hline \multicolumn{9}{|c|}{ Colombia (four registries) } \\
\hline $2000-04$ & $\begin{array}{l}10 \cdot 7 \S \\
(5 \cdot 6-15 \cdot 8)\end{array}$ & $\begin{array}{l}18 \cdot 4 \\
(16 \cdot 0-20 \cdot 9)\end{array}$ & $\begin{array}{l}45 \cdot 0 \\
(40 \cdot 6-49 \cdot 4)\end{array}$ & $\begin{array}{l}38 \cdot 3 \\
(32 \cdot 9-43 \cdot 7)\end{array}$ & $\begin{array}{c}4.85 \\
(1.8-7.9)\end{array}$ & $\begin{array}{c}6 \cdot 45 \\
(3 \cdot 5-9 \cdot 4)\end{array}$ & $\begin{array}{c}9 \cdot 4 \\
(7 \cdot 2-11 \cdot 5)\end{array}$ & $\begin{array}{l}63 \cdot 15 \\
(57 \cdot 0-69 \cdot 1)\end{array}$ \\
\hline 2005-09 & $\begin{array}{c}9 \cdot 5 \\
(4 \cdot 6-14 \cdot 4)\end{array}$ & $\begin{array}{l}17 \cdot 7 \\
(16 \cdot 2-19 \cdot 3)\end{array}$ & $\begin{array}{l}41 \cdot 3 \\
(37 \cdot 9-44 \cdot 7)\end{array}$ & $\begin{array}{l}37 \cdot 2 \\
(32 \cdot 7-41 \cdot 7)\end{array}$ & $\begin{array}{c}5 \cdot 4 \\
(3 \cdot 1-7 \cdot 7)\end{array}$ & $\begin{array}{c}3 \cdot 4 \\
(1 \cdot 6-5 \cdot 2)\end{array}$ & $\begin{array}{l}10 \cdot 5 \\
(8 \cdot 5-12 \cdot 5)\end{array}$ & $\begin{array}{l}71 \cdot 3 \S \\
(66 \cdot 2-76 \cdot 5)\end{array}$ \\
\hline 2010-14 & $\begin{array}{l}10 \cdot 55 \\
(3 \cdot 9-17 \cdot 1)\end{array}$ & $\begin{array}{l}17 \cdot 1 \S \\
(15 \cdot 4-18 \cdot 8)\end{array}$ & $\begin{array}{l}34 \cdot 5 \S \\
(31 \cdot 2-37 \cdot 9)\end{array}$ & $\begin{array}{l}38 \cdot 05 \\
(33 \cdot 2-42 \cdot 8)\end{array}$ & $\begin{array}{c}5 \cdot 2 \S \\
(2 \cdot 7-7 \cdot 8)\end{array}$ & $\begin{array}{c}5 \cdot 35 \\
(2 \cdot 8-7 \cdot 8)\end{array}$ & $\begin{array}{c}8 \cdot 75 \\
(6 \cdot 7-10 \cdot 6)\end{array}$ & $\begin{array}{l}65 \cdot 15 \\
(59 \cdot 2-71 \cdot 1)\end{array}$ \\
\hline \multicolumn{9}{|l|}{ Costa Rica* } \\
\hline $2000-04$ & $\begin{array}{l}35 \cdot 7 \S \\
(26 \cdot 3-45 \cdot 1)\end{array}$ & $\begin{array}{l}48 \cdot 4 \\
(45 \cdot 5-51 \cdot 2)\end{array}$ & $\begin{array}{l}63 \cdot 8 \\
(59 \cdot 5-68 \cdot 1)\end{array}$ & $\begin{array}{l}48 \cdot 4 \\
(41 \cdot 3-55 \cdot 6)\end{array}$ & $\begin{array}{l}39 \cdot 0 \$ \\
(32 \cdot 4-45 \cdot 6)\end{array}$ & $\begin{array}{l}38 \cdot 15 \\
(31 \cdot 3-44 \cdot 8)\end{array}$ & $\begin{array}{l}36 \cdot 25 \\
(31 \cdot 0-41 \cdot 5)\end{array}$ & $\begin{array}{l}82 \cdot 6 \\
(77 \cdot 2-88 \cdot 1)\end{array}$ \\
\hline $2005-09$ & $\begin{array}{l}19 \cdot 25 \\
(11 \cdot 3-27 \cdot 1)\end{array}$ & $\begin{array}{l}38 \cdot 4 \\
(36 \cdot 3-40 \cdot 5)\end{array}$ & $\begin{array}{l}55 \cdot 1 \\
(52 \cdot 2-58 \cdot 0)\end{array}$ & $\begin{array}{l}50 \cdot 2 \\
(45 \cdot 2-55 \cdot 2)\end{array}$ & $\begin{array}{l}23.65 \\
(18 \cdot 5-28 \cdot 7)\end{array}$ & $\begin{array}{l}23 \cdot 85 \\
(19 \cdot 1-28 \cdot 6)\end{array}$ & $\begin{array}{l}22 \cdot 05 \\
(19 \cdot 0-25 \cdot 1)\end{array}$ & $\begin{array}{l}76 \cdot 2 \\
(71 \cdot 5-80 \cdot 8)\end{array}$ \\
\hline $2010-14$ & $\begin{array}{l}20 \cdot 9 \S \\
(14 \cdot 3-27 \cdot 6)\end{array}$ & $\begin{array}{l}40 \cdot 6 \\
(38 \cdot 5-42 \cdot 7)\end{array}$ & $\begin{array}{l}60 \cdot 1 \\
(57 \cdot 4-62 \cdot 8)\end{array}$ & $\begin{array}{l}53 \cdot 9 \\
(49 \cdot 2-58 \cdot 5)\end{array}$ & $\begin{array}{l}24 \cdot 15 \\
(19 \cdot 7-28 \cdot 5)\end{array}$ & $\begin{array}{l}24 \cdot 5 \S \\
(19 \cdot 5-29 \cdot 5)\end{array}$ & $\begin{array}{l}20 \cdot 15 \\
(17 \cdot 1-23 \cdot 1)\end{array}$ & $\begin{array}{l}77 \cdot 2 \\
(72 \cdot 7-81 \cdot 7)\end{array}$ \\
\hline \multicolumn{9}{|l|}{ Cuba* } \\
\hline $2000-04$ &. & $\begin{array}{l}17 \cdot 25 \\
(15 \cdot 7-18 \cdot 7)\end{array}$ & $\begin{array}{l}39 \cdot 8 \S \\
(38 \cdot 3-41 \cdot 2)\end{array}$ & .. & .. & .. & $\begin{array}{c}4 \cdot 5 \$ \\
(3 \cdot 8-5 \cdot 1)\end{array}$ & .. \\
\hline $2005-09$ &. & $\begin{array}{l}25 \cdot 65 \\
(23 \cdot 6-27 \cdot 5)\end{array}$ & $\begin{array}{l}48 \cdot 55 \\
(46 \cdot 9-50 \cdot 1)\end{array}$ & .. & .. & .. & $\begin{array}{l}23 \cdot 25 \\
(22 \cdot 1-24 \cdot 2)\end{array}$ & .. \\
\hline $2010-14$ &. & $\begin{array}{l}35 \cdot 7 \S \\
(32 \cdot 8-38 \cdot 6)\end{array}$ & $\begin{array}{l}63.95 \\
(61 \cdot 6-66 \cdot 2)\end{array}$ & .. & .. & .. & $\begin{array}{l}30 \cdot 15 \\
(28 \cdot 4-31 \cdot 8)\end{array}$ & .. \\
\hline \multicolumn{9}{|c|}{ Ecuador (five registries) } \\
\hline $2000-04$ & $\begin{array}{l}20 \cdot 9 \dagger \\
(9 \cdot 9-31 \cdot 9)\end{array}$ & $\begin{array}{l}17 \cdot 8 \\
(12 \cdot 3-23 \cdot 3)\end{array}$ & $\begin{array}{l}47 \cdot 7 \\
(41 \cdot 0-54 \cdot 4)\end{array}$ & $\begin{array}{l}38 \cdot 7 \\
(30 \cdot 4-47 \cdot 1)\end{array}$ & $\begin{array}{c}8 \cdot 15 \\
(4 \cdot 6-11 \cdot 5)\end{array}$ & $\begin{array}{c}8 \cdot 45 \\
(4 \cdot 7-12 \cdot 2)\end{array}$ & $\begin{array}{c}10 \cdot 15 \\
(7 \cdot 2-13 \cdot 0)\end{array}$ & $\begin{array}{l}59 \cdot 0 \\
(50 \cdot 9-67 \cdot 1)\end{array}$ \\
\hline 2005-09 & $\begin{array}{c}7 \cdot 7 \\
(3 \cdot 5-11 \cdot 9)\end{array}$ & $\begin{array}{l}17 \cdot 4 \\
(12 \cdot 0-22 \cdot 7)\end{array}$ & $\begin{array}{l}46 \cdot 7 \\
(42 \cdot 9-50 \cdot 5)\end{array}$ & $\begin{array}{l}43 \cdot 5 \\
(38 \cdot 8-48 \cdot 2)\end{array}$ & $\begin{array}{c}6 \cdot 35 \\
(4 \cdot 1-8 \cdot 6)\end{array}$ & $\begin{array}{c}9 \cdot 05 \\
(6 \cdot 2-11 \cdot 8)\end{array}$ & $\begin{array}{l}10 \cdot 55 \\
(8 \cdot 5-12 \cdot 4)\end{array}$ & $\begin{array}{l}60 \cdot 5 \\
(54 \cdot 5-66 \cdot 5)\end{array}$ \\
\hline $2010-14$ & $\begin{array}{l}12 \cdot 7 \\
(6 \cdot 7-18 \cdot 7)\end{array}$ & $\begin{array}{l}19 \cdot 1 \\
(13 \cdot 1-25 \cdot 1)\end{array}$ & $\begin{array}{l}47 \cdot 8 \\
(43 \cdot 9-51 \cdot 6)\end{array}$ & $\begin{array}{l}44 \cdot 5 \\
(39 \cdot 2-49 \cdot 8)\end{array}$ & $\begin{array}{c}5 \cdot 95 \\
(3 \cdot 9-7 \cdot 8)\end{array}$ & $\begin{array}{c}8 \cdot 45 \\
(5 \cdot 7-11 \cdot 2)\end{array}$ & $\begin{array}{l}12 \cdot 25 \\
(10 \cdot 0-14 \cdot 4)\end{array}$ & $\begin{array}{l}57 \cdot 9 \\
(51 \cdot 6-64 \cdot 1)\end{array}$ \\
\hline \multicolumn{9}{|l|}{ Guadeloupe* } \\
\hline $2000-04$ &.. & .. & .. & .. & .. &.. &. & .. \\
\hline $2005-09$ & $\begin{array}{c}0.0 \dagger \\
(0.0-0.1)\end{array}$ & $\begin{array}{c}11 \cdot 7 \S \\
(5 \cdot 7-17 \cdot 7)\end{array}$ & $\begin{array}{l}30 \cdot 9 \S \\
(23 \cdot 1-38 \cdot 7)\end{array}$ & $\begin{array}{l}21 \cdot 3 \$ \\
(8 \cdot 8-33 \cdot 8)\end{array}$ & $\begin{array}{c}9.5+5 \\
(0.0-20 \cdot 3)\end{array}$ & $\begin{array}{c}11 \cdot 2+5 \\
(1 \cdot 7-20 \cdot 8)\end{array}$ & $\begin{array}{c}6 \cdot 8 \\
(2 \cdot 3-11 \cdot 2)\end{array}$ & $\begin{array}{l}86 \cdot 2+5 \\
(53 \cdot 0-100 \cdot 0)\end{array}$ \\
\hline $2010-14$ &.. & $\begin{array}{l}11 \cdot 7 \S \\
(6 \cdot 8-16 \cdot 6)\end{array}$ & $\begin{array}{l}26 \cdot 85 \\
(19 \cdot 5-34 \cdot 0)\end{array}$ & $\begin{array}{l}35 \cdot 65 \\
(22 \cdot 4-48 \cdot 8)\end{array}$ & $\begin{array}{c}3 \cdot 7+5 \\
(0 \cdot 0-9 \cdot 2)\end{array}$ &.. & $\begin{array}{c}4.05 \\
(0.2-7 \cdot 7)\end{array}$ & $\begin{array}{c}14 \cdot 1+5 \\
(0 \cdot 0-36 \cdot 7)\end{array}$ \\
\hline \multicolumn{9}{|l|}{ Martinique* $^{*}$} \\
\hline 2000-04 & $\begin{array}{c}4 \cdot 2 \dagger \\
(0 \cdot 4-8 \cdot 1)\end{array}$ & $\begin{array}{l}29 \cdot 7 \\
(24 \cdot 2-35 \cdot 2)\end{array}$ & $\begin{array}{l}57 \cdot 0 \\
(50 \cdot 4-63 \cdot 6)\end{array}$ & $\begin{array}{l}44 \cdot 1 \\
(35 \cdot 1-53 \cdot 1)\end{array}$ & $\begin{array}{l}12 \cdot 0 \\
(5 \cdot 6-18 \cdot 4)\end{array}$ & $\begin{array}{l}13 \cdot 4 \\
(7 \cdot 8-19 \cdot 1)\end{array}$ & $\begin{array}{c}12 \cdot 5 \\
(7 \cdot 6-17 \cdot 4)\end{array}$ & $\begin{array}{l}81 \cdot 8+5 \\
(68 \cdot 5-95 \cdot 1)\end{array}$ \\
\hline 2005-09 & $\begin{array}{c}4 \cdot 9 \\
(1 \cdot 1-8 \cdot 7)\end{array}$ & $\begin{array}{l}33 \cdot 0 \\
(27 \cdot 5-38 \cdot 5)\end{array}$ & $\begin{array}{l}54 \cdot 9 \\
(49 \cdot 5-60 \cdot 3)\end{array}$ & $\begin{array}{l}54 \cdot 0 \\
(46 \cdot 0-62 \cdot 1)\end{array}$ & $\begin{array}{l}12 \cdot 4 \\
(5 \cdot 6-19 \cdot 1)\end{array}$ & $\begin{array}{c}6 \cdot 9 \\
(3 \cdot 2-10 \cdot 5)\end{array}$ & $\begin{array}{l}14 \cdot 7 \\
(10 \cdot 4-18 \cdot 9)\end{array}$ & $\begin{array}{l}86 \cdot 4+5 \\
(74 \cdot 7-98 \cdot 2)\end{array}$ \\
\hline $2010-14$ & $\begin{array}{c}4 \cdot 0 \dagger \\
(1 \cdot 8-6 \cdot 3)\end{array}$ & $\begin{array}{l}32 \cdot 1 \\
(24 \cdot 5-39 \cdot 6)\end{array}$ & $\begin{array}{l}53 \cdot 6 \\
(46 \cdot 9-60 \cdot 3)\end{array}$ & $\begin{array}{l}52 \cdot 0 \\
(42 \cdot 2-61 \cdot 7)\end{array}$ & $\begin{array}{l}15 \cdot 6 \\
(7 \cdot 2-23 \cdot 9)\end{array}$ & $\begin{array}{l}11 \cdot 9 \\
(6 \cdot 5-17 \cdot 3)\end{array}$ & $\begin{array}{l}11 \cdot 8 \\
(6 \cdot 4-17 \cdot 2)\end{array}$ & $\begin{array}{l}77 \cdot 85 \\
(61 \cdot 3-94 \cdot 3)\end{array}$ \\
\hline \multicolumn{9}{|l|}{ Peru (Lima) } \\
\hline 2000-04 &. &. &. & .. & .. & .. & .. & .. \\
\hline $2005-09$ &.$\cdot$ & .. &.. & .. & .. & .. & .. & .. \\
\hline \multirow[t]{2}{*}{ 2010-14 } &.. & .. & $\begin{array}{l}59 \cdot 0 \\
(55 \cdot 8-62 \cdot 1)\end{array}$ & $\begin{array}{l}54 \cdot 8 \\
(50 \cdot 0-59 \cdot 5)\end{array}$ & .. & .. &.. & .. \\
\hline & & & & & & & \multicolumn{2}{|c|}{ (Table 6 continues on next page) } \\
\hline
\end{tabular}




\begin{tabular}{|c|c|c|c|c|c|c|c|c|}
\hline & \multicolumn{6}{|c|}{ Gastrointestinal cancers } & \multirow[t]{2}{*}{ Lung } & \multirow{2}{*}{$\begin{array}{l}\text { Melanoma of } \\
\text { the skin }\end{array}$} \\
\hline & Oesophagus & Stomach & Colon & Rectum & Liver & Pancreas & & \\
\hline \multicolumn{9}{|c|}{ (Continued from previous page) } \\
\hline \multicolumn{9}{|l|}{ Puerto Rico* } \\
\hline 2000-04 & $\begin{array}{l}10 \cdot 9 \\
(8 \cdot 5-13 \cdot 4)\end{array}$ & $\begin{array}{l}26 \cdot 6 \\
(24 \cdot 1-29 \cdot 1)\end{array}$ & $\begin{array}{l}60 \cdot 9 \\
(59 \cdot 3-62 \cdot 5)\end{array}$ & $\begin{array}{l}53 \cdot 9 \\
(50 \cdot 9-57.0)\end{array}$ & $\begin{array}{c}11 \cdot 85 \\
(9 \cdot 0-14 \cdot 6)\end{array}$ & $\begin{array}{c}8.85 \\
(6 \cdot 3-11 \cdot 2)\end{array}$ & $\begin{array}{l}14 \cdot 7 \S \\
(13 \cdot 1-16 \cdot 3)\end{array}$ & $\begin{array}{l}73 \cdot 3 \\
(68 \cdot 2-78 \cdot 4)\end{array}$ \\
\hline 2005-09 & $\begin{array}{l}13 \cdot 0 \\
(10 \cdot 1-15 \cdot 9)\end{array}$ & $\begin{array}{l}29 \cdot 9 \\
(27 \cdot 3-32 \cdot 5)\end{array}$ & $\begin{array}{l}62 \cdot 1 \\
(60 \cdot 6-63 \cdot 5)\end{array}$ & $\begin{array}{l}59 \cdot 4 \\
(56 \cdot 6-62 \cdot 3)\end{array}$ & $\begin{array}{c}8 \cdot 75 \\
(6 \cdot 9-10 \cdot 5)\end{array}$ & $\begin{array}{c}9.05 \\
(6 \cdot 9-11 \cdot 1)\end{array}$ & $\begin{array}{l}16 \cdot 0 \\
(14 \cdot 5-17 \cdot 5)\end{array}$ & $\begin{array}{l}75 \cdot 4 \\
(70 \cdot 6-80 \cdot 1)\end{array}$ \\
\hline 2010-14 & $\begin{array}{l}20 \cdot 4 \\
(14 \cdot 8-25 \cdot 9)\end{array}$ & $\begin{array}{l}34 \cdot 6 \\
(30 \cdot 3-39 \cdot 0)\end{array}$ & $\begin{array}{l}63 \cdot 4 \\
(61 \cdot 1-65 \cdot 7)\end{array}$ & $\begin{array}{l}59 \cdot 0 \\
(54 \cdot 6-63 \cdot 4)\end{array}$ & $\begin{array}{l}14 \cdot 25 \\
(10 \cdot 4-18 \cdot 0)\end{array}$ & $\begin{array}{c}10 \cdot 25 \\
(7 \cdot 1-13 \cdot 3)\end{array}$ & $\begin{array}{l}17 \cdot 6 \\
(14 \cdot 9-20 \cdot 3)\end{array}$ & $\begin{array}{l}75 \cdot 1 \\
(68 \cdot 2-81 \cdot 9)\end{array}$ \\
\hline \multicolumn{9}{|l|}{ Uruguay* } \\
\hline 2000-04 &.$\cdot$ & .. &.. &.$\cdot$ &.. & .. &.. &.$\cdot$ \\
\hline 2005-09 & $\begin{array}{l}12 \cdot 0 \S \\
(8 \cdot 4-15 \cdot 6)\end{array}$ & $\begin{array}{l}20 \cdot 5 \\
(17 \cdot 5-23 \cdot 5)\end{array}$ & $\begin{array}{l}57 \cdot 7 \\
(55 \cdot 0-60 \cdot 4)\end{array}$ & $\begin{array}{l}50 \cdot 5 \\
(46 \cdot 3-54 \cdot 6)\end{array}$ & $\begin{array}{l}12 \cdot 05 \\
(4 \cdot 4-19 \cdot 7)\end{array}$ & $\begin{array}{c}4 \cdot 35 \\
(2 \cdot 3-6 \cdot 3)\end{array}$ & $\begin{array}{c}9.9 \Omega \\
(8 \cdot 3-11 \cdot 5)\end{array}$ &. \\
\hline 2010-14 & $\begin{array}{c}8.05 \\
(5 \cdot 3-10 \cdot 7)\end{array}$ & $\begin{array}{l}18 \cdot 5 \S \\
(15 \cdot 4-21 \cdot 5)\end{array}$ & $\begin{array}{c}53 \cdot 5 \\
(49 \cdot 2-57 \cdot 7)\end{array}$ & $\begin{array}{l}50 \cdot 1 \\
(44 \cdot 8-55 \cdot 4)\end{array}$ & $\begin{array}{l}14 \cdot 38 \\
(8 \cdot 0-20 \cdot 6)\end{array}$ & $\begin{array}{c}4.45 \\
(3 \cdot 0-5 \cdot 8)\end{array}$ & $\begin{array}{c}9.05 \\
(7 \cdot 4-10 \cdot 6)\end{array}$ &. \\
\hline \multicolumn{9}{|c|}{ America (North) } \\
\hline \multicolumn{9}{|c|}{ Canada (nine registries) } \\
\hline 2000-04 & $\begin{array}{l}14 \cdot 5 \\
(13 \cdot 4-15 \cdot 6)\end{array}$ & $\begin{array}{l}25 \cdot 1 \\
(24 \cdot 2-26 \cdot 0)\end{array}$ & $\begin{array}{l}61 \cdot 6 \\
(61 \cdot 1-62 \cdot 1)\end{array}$ & $\begin{array}{l}61 \cdot 9 \\
(61 \cdot 1-62 \cdot 8)\end{array}$ & $\begin{array}{l}17 \cdot 4 \\
(16 \cdot 2-18 \cdot 5)\end{array}$ & $\begin{array}{c}7 \cdot 9 \\
(7 \cdot 3-8 \cdot 5)\end{array}$ & $\begin{array}{l}16 \cdot 3 \\
(15 \cdot 9-16 \cdot 6)\end{array}$ & $\begin{array}{l}87 \cdot 9 \\
(87 \cdot 3-88 \cdot 6)\end{array}$ \\
\hline 2005-09 & $\begin{array}{l}14 \cdot 7 \\
(13 \cdot 7-15 \cdot 7)\end{array}$ & $\begin{array}{l}26 \cdot 6 \\
(25 \cdot 7-27 \cdot 5)\end{array}$ & $\begin{array}{l}65 \cdot 7 \\
(65 \cdot 2-66 \cdot 2)\end{array}$ & $\begin{array}{l}65 \cdot 5 \\
(64 \cdot 7-66 \cdot 3)\end{array}$ & $\begin{array}{l}19 \cdot 2 \\
(18 \cdot 2-20 \cdot 2)\end{array}$ & $\begin{array}{c}9.4 \\
(8.8-10.0)\end{array}$ & $\begin{array}{l}18 \cdot 5 \\
(18 \cdot 1-18 \cdot 8)\end{array}$ & $\begin{array}{l}88 \cdot 5 \\
(88 \cdot 0-89 \cdot 1)\end{array}$ \\
\hline 2010-14 & $\begin{array}{l}16 \cdot 1 \\
(15 \cdot 1-17 \cdot 1)\end{array}$ & $\begin{array}{l}29 \cdot 6 \\
(28 \cdot 6-30 \cdot 5)\end{array}$ & $\begin{array}{l}67 \cdot 0 \\
(66 \cdot 5-67 \cdot 5)\end{array}$ & $\begin{array}{l}66 \cdot 8 \\
(66 \cdot 0-67 \cdot 5)\end{array}$ & $\begin{array}{l}18 \cdot 7 \\
(17 \cdot 8-19 \cdot 7)\end{array}$ & $\begin{array}{l}10 \cdot 8 \\
(10 \cdot 2-11 \cdot 4)\end{array}$ & $\begin{array}{l}20 \cdot 6 \\
(20 \cdot 2-20 \cdot 9)\end{array}$ & $\begin{array}{l}89 \cdot 1 \\
(88 \cdot 6-89 \cdot 6)\end{array}$ \\
\hline \multicolumn{9}{|c|}{ USA (48 registries) } \\
\hline 2000-04 & $\begin{array}{l}16 \cdot 5 \\
(16 \cdot 1-16 \cdot 8)\end{array}$ & $\begin{array}{l}26 \cdot 2 \\
(25 \cdot 8-26 \cdot 5)\end{array}$ & $\begin{array}{l}64 \cdot 7 \\
(64 \cdot 5-64 \cdot 9)\end{array}$ & $\begin{array}{l}63 \cdot 9 \\
(63 \cdot 5-64 \cdot 2)\end{array}$ & $\begin{array}{l}12 \cdot 5 \\
(12 \cdot 2-12 \cdot 8)\end{array}$ & $\begin{array}{c}7 \cdot 2 \\
(7 \cdot 0-7 \cdot 4)\end{array}$ & $\begin{array}{l}17 \cdot 0 \\
(16 \cdot 9-17 \cdot 1)\end{array}$ & $\begin{array}{l}88 \cdot 9 \\
(88 \cdot 7-89 \cdot 1)\end{array}$ \\
\hline 2005-09 & $\begin{array}{l}18 \cdot 7 \\
(18 \cdot 4-19 \cdot 1)\end{array}$ & $\begin{array}{l}30 \cdot 1 \\
(29 \cdot 7-30 \cdot 4)\end{array}$ & $\begin{array}{l}65 \cdot 5 \\
(65 \cdot 3-65 \cdot 7)\end{array}$ & $\begin{array}{l}64 \cdot 5 \\
(64 \cdot 1-64 \cdot 8)\end{array}$ & $\begin{array}{l}15 \cdot 6 \\
(15 \cdot 3-15 \cdot 9)\end{array}$ & $\begin{array}{c}8.9 \\
(8.7-9.1)\end{array}$ & $\begin{array}{l}19 \cdot 4 \\
(19 \cdot 3-19 \cdot 5)\end{array}$ & $\begin{array}{l}90 \cdot 4 \\
(90 \cdot 2-90 \cdot 6)\end{array}$ \\
\hline 2010-14 & $\begin{array}{l}20 \cdot 0 \\
(19 \cdot 6-20 \cdot 4)\end{array}$ & $\begin{array}{l}33 \cdot 1 \\
(32 \cdot 7-33 \cdot 4)\end{array}$ & $\begin{array}{l}64 \cdot 9 \\
(64.7-65 \cdot 1)\end{array}$ & $\begin{array}{l}64 \cdot 1 \\
(63 \cdot 7-64 \cdot 4)\end{array}$ & $\begin{array}{l}17 \cdot 4 \\
(17 \cdot 1-17 \cdot 7)\end{array}$ & $\begin{array}{l}11 \cdot 5 \\
(11 \cdot 3-11 \cdot 7)\end{array}$ & $\begin{array}{l}21 \cdot 2 \\
(21 \cdot 1-21 \cdot 3)\end{array}$ & $\begin{array}{l}90 \cdot 8 \\
(90 \cdot 6-91 \cdot 0)\end{array}$ \\
\hline \multicolumn{9}{|l|}{ Asia } \\
\hline \multicolumn{9}{|c|}{ China (21 registries) } \\
\hline 2000-04 & $\begin{array}{l}22 \cdot 9 \\
(22 \cdot 0-23 \cdot 9)\end{array}$ & $\begin{array}{l}30 \cdot 2 \\
(29 \cdot 3-31 \cdot 1)\end{array}$ & $\begin{array}{l}51 \cdot 4 \\
(49 \cdot 6-53 \cdot 3)\end{array}$ & $\begin{array}{l}49 \cdot 5 \\
(47 \cdot 5-51 \cdot 4)\end{array}$ & $\begin{array}{l}11 \cdot 7 \\
(10 \cdot 9-12 \cdot 5)\end{array}$ & $\begin{array}{l}14 \cdot 4 \\
(12 \cdot 8-16 \cdot 0)\end{array}$ & $\begin{array}{l}18 \cdot 7 \\
(18 \cdot 0-19 \cdot 4)\end{array}$ & $\begin{array}{l}35 \cdot 6 \\
(25 \cdot 8-45 \cdot 4)\end{array}$ \\
\hline 2005-09 & $\begin{array}{l}27 \cdot 1 \\
(26 \cdot 5-27 \cdot 7)\end{array}$ & $\begin{array}{l}33 \cdot 2 \\
(32 \cdot 7-33 \cdot 7)\end{array}$ & $\begin{array}{l}55 \cdot 6 \\
(54 \cdot 6-56 \cdot 5)\end{array}$ & $\begin{array}{l}52 \cdot 5 \\
(51 \cdot 5-53 \cdot 6)\end{array}$ & $\begin{array}{c}11 \cdot 6 \\
(11 \cdot 1-12 \cdot 0)\end{array}$ & $\begin{array}{l}10 \cdot 2 \\
(9 \cdot 4-11 \cdot 0)\end{array}$ & $\begin{array}{l}17 \cdot 7 \\
(17 \cdot 4-18 \cdot 1)\end{array}$ & $\begin{array}{l}45 \cdot 5 \\
(40 \cdot 8-50 \cdot 2)\end{array}$ \\
\hline 2010-14 & $\begin{array}{l}29 \cdot 7 \\
(29 \cdot 0-30 \cdot 4)\end{array}$ & $\begin{array}{l}35 \cdot 9 \\
(35 \cdot 3-36 \cdot 5)\end{array}$ & $\begin{array}{l}57 \cdot 6 \\
(56 \cdot 6-58.6)\end{array}$ & $\begin{array}{l}56 \cdot 9 \\
(55 \cdot 8-58 \cdot 0)\end{array}$ & $\begin{array}{l}14 \cdot 1 \\
(13 \cdot 6-14 \cdot 7)\end{array}$ & $\begin{array}{c}9.9 \\
(9 \cdot 1-10 \cdot 7)\end{array}$ & $\begin{array}{l}19 \cdot 8 \\
(19 \cdot 4-20 \cdot 2)\end{array}$ & $\begin{array}{l}49 \cdot 6 \\
(44 \cdot 5-54 \cdot 6)\end{array}$ \\
\hline \multicolumn{9}{|l|}{ Cyprus* } \\
\hline 2000-04 &.. & $\begin{array}{l}36 \cdot 6 \dagger \\
(22 \cdot 2-51 \cdot 1)\end{array}$ & $\begin{array}{l}70 \cdot 75 \\
(61 \cdot 8-79 \cdot 6)\end{array}$ & $\begin{array}{l}61 \cdot 9+5 \\
(43 \cdot 0-80 \cdot 8)\end{array}$ & $\begin{array}{c}8.4+5 \\
(0.0-21 \cdot 0)\end{array}$ & $\begin{array}{c}12 \cdot 8+5 \\
(1.7-23 \cdot 9)\end{array}$ & $\begin{array}{l}18 \cdot 3 \\
(12 \cdot 2-24 \cdot 3)\end{array}$ & $\begin{array}{l}89 \cdot 0+\$ \\
(73 \cdot 6-100 \cdot 0)\end{array}$ \\
\hline 2005-09 & $\begin{array}{l}47 \cdot 2+5 \\
(30 \cdot 5-63 \cdot 8)\end{array}$ & $\begin{array}{l}28 \cdot 15 \\
(22 \cdot 9-33 \cdot 3)\end{array}$ & $\begin{array}{l}67 \cdot 65 \\
(63 \cdot 9-71 \cdot 4)\end{array}$ & $\begin{array}{l}78 \cdot 38 \\
(71 \cdot 3-85 \cdot 4)\end{array}$ & $\begin{array}{l}13 \cdot 3+\$ \\
(6 \cdot 3-20 \cdot 2)\end{array}$ & $\begin{array}{c}8.05 \\
(4.5-11 \cdot 6)\end{array}$ & $\begin{array}{l}18 \cdot 2 \\
(15 \cdot 6-20 \cdot 8)\end{array}$ & $\begin{array}{l}82 \cdot 15 \\
(75 \cdot 8-88 \cdot 4)\end{array}$ \\
\hline 2010-14 & $\begin{array}{l}39 \cdot 0+5 \\
(21 \cdot 8-56 \cdot 3)\end{array}$ & $\begin{array}{l}35 \cdot 65 \\
(30 \cdot 0-41 \cdot 2)\end{array}$ & $\begin{array}{l}72 \cdot 15 \\
(68 \cdot 5-75 \cdot 8)\end{array}$ & $\begin{array}{l}75 \cdot 9 \Omega \\
(69 \cdot 6-82 \cdot 2)\end{array}$ & $\begin{array}{l}10 \cdot 6 \varsigma \\
(5 \cdot 8-15 \cdot 5)\end{array}$ & $\begin{array}{c}11.65 \\
(7 \cdot 7-15 \cdot 5)\end{array}$ & $\begin{array}{l}18 \cdot 7 \\
(16 \cdot 2-21 \cdot 2)\end{array}$ & $\begin{array}{l}79.05 \\
(73 \cdot 1-85 \cdot 0)\end{array}$ \\
\hline \multicolumn{9}{|l|}{ Hong Kong* } \\
\hline 2000-04 &.. &. &. &.. &.. & .. &.. &.. \\
\hline 2005-09 & & & $\begin{array}{l}56 \cdot 1 \\
(55 \cdot 1-57 \cdot 1)\end{array}$ & $\begin{array}{l}57 \cdot 3 \\
(55 \cdot 8-58 \cdot 7)\end{array}$ &.. & & &.. \\
\hline \multirow[t]{2}{*}{ 2010-14 } &.. &. & $\begin{array}{l}56 \cdot 4 \\
(55 \cdot 4-57 \cdot 3)\end{array}$ & $\begin{array}{l}58 \cdot 0 \\
(56 \cdot 6-59 \cdot 4)\end{array}$ &. &.. &. &. \\
\hline & & & & & & & \multicolumn{2}{|c|}{ (Table 6 continues on next page) } \\
\hline
\end{tabular}




\begin{tabular}{|c|c|c|c|c|c|c|c|c|}
\hline & \multicolumn{6}{|c|}{ Gastrointestinal cancers } & \multirow[t]{2}{*}{ Lung } & \multirow{2}{*}{$\begin{array}{l}\text { Melanoma of } \\
\text { the skin }\end{array}$} \\
\hline & Oesophagus & Stomach & Colon & Rectum & Liver & Pancreas & & \\
\hline \multicolumn{9}{|c|}{ (Continued from previous page) } \\
\hline \multicolumn{9}{|c|}{ India (two registries) } \\
\hline $2000-04$ & $\begin{array}{c}2.9 \dagger \\
(0.0-6 \cdot 4)\end{array}$ & $\begin{array}{c}6 \cdot 4 \\
(2 \cdot 5-10 \cdot 3)\end{array}$ & $\begin{array}{l}46 \cdot 9 \dagger \\
(31 \cdot 9-61 \cdot 8)\end{array}$ & $\begin{array}{l}36 \cdot 9 \dagger \\
(16 \cdot 1-57 \cdot 8)\end{array}$ & $\begin{array}{c}2.4 \dagger \\
(0.0-6 \cdot 0)\end{array}$ & $\begin{array}{c}4.2 \dagger \\
(0 \cdot 0-10 \cdot 5)\end{array}$ & $\begin{array}{c}6 \cdot 9 \\
(2 \cdot 3-11 \cdot 5)\end{array}$ &.$\cdot$ \\
\hline 2005-09 & $\begin{array}{c}3 \cdot 4 \\
(0.7-6 \cdot 0)\end{array}$ & $\begin{array}{c}9 \cdot 8 \\
(3 \cdot 4-16 \cdot 3)\end{array}$ & $\begin{array}{l}33 \cdot 8 \dagger \\
(21 \cdot 1-46 \cdot 4)\end{array}$ & $\begin{array}{l}33 \cdot 6 \\
(20 \cdot 8-46 \cdot 3)\end{array}$ & $\begin{array}{c}3 \cdot 7 \\
(0.0-7 \cdot 7)\end{array}$ & $\begin{array}{c}5 \cdot 3 \dagger \\
(0 \cdot 0-11 \cdot 4)\end{array}$ & $\begin{array}{c}3 \cdot 5 \\
(1 \cdot 4-5 \cdot 7)\end{array}$ &.$\cdot$ \\
\hline $2010-14$ & $\begin{array}{c}4 \cdot 1 \\
(0 \cdot 0-8 \cdot 5)\end{array}$ & $\begin{array}{c}8 \cdot 9 \\
(4 \cdot 0-13 \cdot 9)\end{array}$ & $\begin{array}{l}38 \cdot 9 \\
(24 \cdot 6-53 \cdot 3)\end{array}$ & $\begin{array}{l}30 \cdot 0 \\
(20 \cdot 5-39 \cdot 5)\end{array}$ & $\begin{array}{c}6 \cdot 3 \dagger \\
(0.6-11 \cdot 9)\end{array}$ & $\begin{array}{c}5 \cdot 6 \dagger \\
(0.0-11 \cdot 6)\end{array}$ & $\begin{array}{c}3 \cdot 7 \\
(1 \cdot 6-5 \cdot 7)\end{array}$ & $\begin{array}{l}61 \cdot 5 \dagger \\
(27 \cdot 3-95 \cdot 8)\end{array}$ \\
\hline \multicolumn{9}{|c|}{ Iran (Golestan) } \\
\hline $2000-04$ &.. &.. &.$\cdot$ &.. & .. &.$\cdot$ &.$\cdot$ &.. \\
\hline 2005-09 & $\begin{array}{c}7 \cdot 4 \\
(4 \cdot 8-10 \cdot 0)\end{array}$ & $\begin{array}{c}5 \cdot 7 \\
(3 \cdot 3-8 \cdot 0)\end{array}$ & $\begin{array}{l}29 \cdot 1 \\
(20 \cdot 2-38 \cdot 0)\end{array}$ & $\begin{array}{l}26 \cdot 2 \dagger \\
(14 \cdot 1-38 \cdot 3)\end{array}$ & .. &.$\cdot$ &.$\cdot$ &.$\cdot$ \\
\hline 2010-14 &. & .. &.. &.. & .. &.$\cdot$ &.$\cdot$ &.. \\
\hline \multicolumn{9}{|l|}{ Israel* } \\
\hline 2000-04 & $\begin{array}{l}17 \cdot 9 \\
(14 \cdot 3-21 \cdot 5)\end{array}$ & $\begin{array}{l}29 \cdot 3 \\
(27 \cdot 6-31 \cdot 1)\end{array}$ & $\begin{array}{l}66 \cdot 5 \\
(65 \cdot 4-67 \cdot 6)\end{array}$ & $\begin{array}{l}62 \cdot 7 \\
(60 \cdot 6-64 \cdot 7)\end{array}$ & $\begin{array}{l}15 \cdot 15 \\
(12 \cdot 2-17 \cdot 9)\end{array}$ & $\begin{array}{c}7 \cdot 65 \\
(6 \cdot 4-8 \cdot 8)\end{array}$ & $\begin{array}{l}20 \cdot 8 \\
(19 \cdot 8-21 \cdot 8)\end{array}$ & $\begin{array}{l}85 \cdot 6 \\
(84 \cdot 2-87 \cdot 0)\end{array}$ \\
\hline 2005-09 & $\begin{array}{l}22 \cdot 4 \\
(18 \cdot 6-26 \cdot 2)\end{array}$ & $\begin{array}{l}29 \cdot 8 \\
(28 \cdot 1-31 \cdot 5)\end{array}$ & $\begin{array}{l}71 \cdot 4 \\
(70 \cdot 3-72 \cdot 4)\end{array}$ & $\begin{array}{l}67 \cdot 3 \\
(65 \cdot 3-69 \cdot 3)\end{array}$ & $\begin{array}{l}15 \cdot 6 \$ \\
(13 \cdot 1-18 \cdot 2)\end{array}$ & $\begin{array}{c}9 \cdot 25 \\
(8 \cdot 0-10 \cdot 5)\end{array}$ & $\begin{array}{l}23 \cdot 8 \\
(22 \cdot 8-24 \cdot 8)\end{array}$ & $\begin{array}{l}88 \cdot 4 \\
(87 \cdot 2-89 \cdot 7)\end{array}$ \\
\hline 2010-14 & $\begin{array}{l}25 \cdot 8 \\
(21 \cdot 4-30 \cdot 2)\end{array}$ & $\begin{array}{l}32 \cdot 3 \\
(30 \cdot 4-34 \cdot 3)\end{array}$ & $\begin{array}{l}71 \cdot 7 \\
(70 \cdot 6-72 \cdot 9)\end{array}$ & $\begin{array}{l}67.8 \\
(65.6-70 \cdot 0)\end{array}$ & $\begin{array}{l}18 \cdot 9 \S \\
(15 \cdot 6-22 \cdot 2)\end{array}$ & $\begin{array}{l}12 \cdot 45 \\
(10 \cdot 8-14 \cdot 0)\end{array}$ & $\begin{array}{l}26 \cdot 6 \\
(25 \cdot 4-27 \cdot 7)\end{array}$ & $\begin{array}{l}87 \cdot 4 \\
(86 \cdot 1-88 \cdot 8)\end{array}$ \\
\hline \multicolumn{9}{|c|}{ Japan (16 registries) } \\
\hline $2000-04$ & $\begin{array}{l}27 \cdot 7 \\
(26 \cdot 4-29 \cdot 0)\end{array}$ & $\begin{array}{l}50 \cdot 5 \\
(50 \cdot 0-50 \cdot 9)\end{array}$ & $\begin{array}{l}63 \cdot 4 \\
(62 \cdot 7-64 \cdot 0)\end{array}$ & $\begin{array}{l}58 \cdot 6 \\
(57 \cdot 6-59.5)\end{array}$ & $\begin{array}{l}25 \cdot 75 \\
(25 \cdot 1-26 \cdot 3)\end{array}$ & $\begin{array}{c}6 \cdot 9 \$ \\
(6 \cdot 4-7 \cdot 4)\end{array}$ & $\begin{array}{l}29 \cdot 3 \\
(28 \cdot 1-30 \cdot 5)\end{array}$ & $\begin{array}{l}68 \cdot 9 \\
(65 \cdot 1-72 \cdot 8)\end{array}$ \\
\hline 2005-09 & $\begin{array}{l}33 \cdot 3 \\
(32 \cdot 3-34 \cdot 2)\end{array}$ & $\begin{array}{l}57 \cdot 6 \\
(57 \cdot 3-57 \cdot 9)\end{array}$ & $\begin{array}{l}66 \cdot 8 \\
(66 \cdot 3-67 \cdot 3)\end{array}$ & $\begin{array}{l}64 \cdot 0 \\
(63 \cdot 3-64 \cdot 6)\end{array}$ & $\begin{array}{l}28.6 \$ \\
(28 \cdot 1-29 \cdot 1)\end{array}$ & $\begin{array}{c}7 \cdot 65 \\
(7 \cdot 2-7 \cdot 9)\end{array}$ & $\begin{array}{l}29 \cdot 3 \\
(28 \cdot 9-29 \cdot 7)\end{array}$ & $\begin{array}{l}68 \cdot 3 \\
(65 \cdot 6-71 \cdot 0)\end{array}$ \\
\hline 2010-14 & $\begin{array}{l}36 \cdot 0 \\
(34 \cdot 8-37 \cdot 3)\end{array}$ & $\begin{array}{l}60 \cdot 3 \\
(59 \cdot 9-60 \cdot 7)\end{array}$ & $\begin{array}{l}67 \cdot 8 \\
(67 \cdot 3-68 \cdot 4)\end{array}$ & $\begin{array}{l}64 \cdot 8 \\
(64 \cdot 0-65 \cdot 7)\end{array}$ & $\begin{array}{l}30 \cdot 15 \\
(29 \cdot 5-30 \cdot 6)\end{array}$ & $\begin{array}{c}8 \cdot 35 \\
(7 \cdot 8-8 \cdot 7)\end{array}$ & $\begin{array}{l}32 \cdot 9 \\
(32 \cdot 3-33 \cdot 4)\end{array}$ & $\begin{array}{l}69 \cdot 0 \\
(66 \cdot 0-72 \cdot 0)\end{array}$ \\
\hline \multicolumn{9}{|l|}{ Jordan* } \\
\hline $2000-04$ & $\begin{array}{l}52 \cdot 6 \$ \\
(41 \cdot 3-63 \cdot 9)\end{array}$ & $\begin{array}{l}76 \cdot 15 \\
(69 \cdot 7-82 \cdot 5)\end{array}$ & $\begin{array}{l}86.05 \\
(81.6-90.5)\end{array}$ & $\begin{array}{l}79 \cdot 95 \\
(71 \cdot 1-88 \cdot 8)\end{array}$ & $\begin{array}{l}71 \cdot 5 \S \\
(58 \cdot 1-84 \cdot 9)\end{array}$ & $\begin{array}{l}66 \cdot 95 \\
(52 \cdot 0-81 \cdot 8)\end{array}$ & $\begin{array}{l}42 \cdot 95 \\
(38 \cdot 3-47 \cdot 4)\end{array}$ & $\begin{array}{l}68 \cdot 5+5 \\
(56 \cdot 4-80 \cdot 6)\end{array}$ \\
\hline 2005-09 & $\begin{array}{l}53 \cdot 95 \\
(43 \cdot 0-64 \cdot 8)\end{array}$ & $\begin{array}{l}64 \cdot 85 \\
(58 \cdot 4-71 \cdot 3)\end{array}$ & $\begin{array}{l}80 \cdot 85 \\
(77 \cdot 0-84 \cdot 7)\end{array}$ & $\begin{array}{l}76 \cdot 25 \\
(67 \cdot 1-85 \cdot 3)\end{array}$ & $\begin{array}{l}64 \cdot 3 \S \\
(54 \cdot 5-74 \cdot 1)\end{array}$ & $\begin{array}{l}32 \cdot 5 \S \\
(25 \cdot 6-39 \cdot 4)\end{array}$ & $\begin{array}{l}44 \cdot 15 \\
(39 \cdot 4-48 \cdot 7)\end{array}$ & $\begin{array}{l}63 \cdot 1+5 \\
(49 \cdot 3-76 \cdot 9)\end{array}$ \\
\hline $2010-14$ & $\begin{array}{l}41 \cdot 15 \\
(30 \cdot 4-51 \cdot 9)\end{array}$ & $\begin{array}{l}55 \cdot 75 \\
(48 \cdot 7-62 \cdot 6)\end{array}$ & $\begin{array}{l}76 \cdot 15 \\
(72 \cdot 1-80 \cdot 1)\end{array}$ & $\begin{array}{l}73 \cdot 25 \\
(65 \cdot 0-81 \cdot 4)\end{array}$ & $\begin{array}{l}40 \cdot 0 \Omega \\
(28 \cdot 6-51 \cdot 3)\end{array}$ & $\begin{array}{l}24 \cdot 05 \\
(18 \cdot 7-29 \cdot 3)\end{array}$ & $\begin{array}{l}28 \cdot 3 \S \\
(24 \cdot 9-31 \cdot 6)\end{array}$ & $\begin{array}{l}55 \cdot 95 \\
(41 \cdot 5-70 \cdot 4)\end{array}$ \\
\hline \multicolumn{9}{|l|}{ Korea* } \\
\hline $2000-04$ & $\begin{array}{l}18 \cdot 6 \\
(17 \cdot 6-19 \cdot 6)\end{array}$ & $\begin{array}{l}48 \cdot 6 \\
(48 \cdot 2-48 \cdot 9)\end{array}$ & $\begin{array}{l}60 \cdot 5 \\
(59 \cdot 9-61 \cdot 2)\end{array}$ & $\begin{array}{l}60 \cdot 8 \\
(60 \cdot 0-61 \cdot 6)\end{array}$ & $\begin{array}{l}15 \cdot 3 \\
(15 \cdot 0-15 \cdot 7)\end{array}$ & $\begin{array}{c}7 \cdot 6 \\
(7 \cdot 2-8 \cdot 1)\end{array}$ & $\begin{array}{l}15 \cdot 3 \\
(15 \cdot 0-15 \cdot 6)\end{array}$ & $\begin{array}{l}51 \cdot 4 \\
(48 \cdot 3-54 \cdot 4)\end{array}$ \\
\hline 2005-09 & $\begin{array}{l}26 \cdot 9 \\
(25 \cdot 8-28 \cdot 0)\end{array}$ & $\begin{array}{l}61 \cdot 1 \\
(60 \cdot 8-61 \cdot 5)\end{array}$ & $\begin{array}{l}68 \cdot 1 \\
(67 \cdot 6-68 \cdot 6)\end{array}$ & $\begin{array}{l}68 \cdot 1 \\
(67 \cdot 5-68 \cdot 7)\end{array}$ & $\begin{array}{l}22 \cdot 4 \\
(22 \cdot 1-22 \cdot 8)\end{array}$ & $\begin{array}{c}8.4 \\
(8 \cdot 0-8 \cdot 9)\end{array}$ & $\begin{array}{l}19 \cdot 9 \\
(19 \cdot 6-20 \cdot 2)\end{array}$ & $\begin{array}{l}55 \cdot 3 \\
(52 \cdot 8-57 \cdot 7)\end{array}$ \\
\hline 2010-14 & $\begin{array}{l}31 \cdot 3 \\
(30 \cdot 3-32 \cdot 4)\end{array}$ & $\begin{array}{l}68 \cdot 9 \\
(68 \cdot 6-69 \cdot 2)\end{array}$ & $\begin{array}{l}71 \cdot 8 \\
(71 \cdot 4-72 \cdot 2)\end{array}$ & $\begin{array}{l}71 \cdot 1 \\
(70 \cdot 6-71 \cdot 7)\end{array}$ & $\begin{array}{l}27 \cdot 2 \\
(26 \cdot 8-27 \cdot 6)\end{array}$ & $\begin{array}{l}10 \cdot 5 \\
(10 \cdot 0-10 \cdot 9)\end{array}$ & $\begin{array}{l}25 \cdot 1 \\
(24 \cdot 8-25 \cdot 4)\end{array}$ & $\begin{array}{l}59 \cdot 9 \\
(57 \cdot 5-62 \cdot 2)\end{array}$ \\
\hline \multicolumn{9}{|l|}{ Kuwait* } \\
\hline $2000-04$ & $\begin{array}{c}17 \cdot 6+5 \\
(2 \cdot 9-32 \cdot 4)\end{array}$ & $\begin{array}{l}15 \cdot 0 \\
(7 \cdot 1-22 \cdot 9)\end{array}$ & $\begin{array}{l}64 \cdot 8 \\
(53 \cdot 1-76 \cdot 5)\end{array}$ & $\begin{array}{l}59 \cdot 3 \\
(48 \cdot 1-70 \cdot 4)\end{array}$ & $\begin{array}{c}11 \cdot 4+5 \\
(3 \cdot 5-19 \cdot 2)\end{array}$ & $\begin{array}{c}11 \cdot 2+5 \\
(3 \cdot 1-19 \cdot 3)\end{array}$ & $\begin{array}{l}13 \cdot 3 \\
(8 \cdot 9-17 \cdot 7)\end{array}$ & .. \\
\hline 2005-09 & $\begin{array}{c}9 \cdot 5^{\dagger} \\
(0 \cdot 0-20 \cdot 6)\end{array}$ & $\begin{array}{c}13 \cdot 4 \\
(7 \cdot 1-19 \cdot 7)\end{array}$ & $\begin{array}{l}50 \cdot 2 \\
(42 \cdot 7-57 \cdot 7)\end{array}$ & $\begin{array}{l}53 \cdot 3 \\
(42 \cdot 4-64 \cdot 2)\end{array}$ & $\begin{array}{c}12 \cdot 4 \dagger \\
(5 \cdot 8-19 \cdot 1)\end{array}$ & $\begin{array}{c}7 \cdot 0 \\
(3 \cdot 0-11 \cdot 0)\end{array}$ & $\begin{array}{l}16 \cdot 3 \\
(11 \cdot 1-21 \cdot 5)\end{array}$ & .. \\
\hline 2010-14 & $\begin{array}{l}25 \cdot 4 \dagger \\
(10 \cdot 5-40 \cdot 4)\end{array}$ & $\begin{array}{l}22 \cdot 4 \\
(12 \cdot 6-32 \cdot 3)\end{array}$ & $\begin{array}{l}58 \cdot 5 \\
(49 \cdot 4-67 \cdot 7)\end{array}$ & $\begin{array}{l}58 \cdot 2 \\
(48 \cdot 5-67 \cdot 9)\end{array}$ & $\begin{array}{l}18 \cdot 6 \\
(9 \cdot 8-27 \cdot 3)\end{array}$ & $\begin{array}{l}23 \cdot 6 \\
(12 \cdot 0-35 \cdot 2)\end{array}$ & $\begin{array}{l}13 \cdot 4 \\
(8 \cdot 8-18 \cdot 0)\end{array}$ & .. \\
\hline \multicolumn{9}{|c|}{ Malaysia (Penang) } \\
\hline $2000-04$ &.. &.$\cdot$ &.$\cdot$ &.. & .. & .. & .. & .. \\
\hline 2005-09 & $\begin{array}{l}13 \cdot 2 \\
(6 \cdot 5-19 \cdot 9)\end{array}$ & $\begin{array}{l}23 \cdot 2 \\
(17 \cdot 5-29 \cdot 0)\end{array}$ & $\begin{array}{l}54.9 \\
(49 \cdot 2-60 \cdot 6)\end{array}$ & $\begin{array}{l}37 \cdot 3 \\
(30 \cdot 1-44 \cdot 5)\end{array}$ & $\begin{array}{c}6 \cdot 4 \\
(3 \cdot 7-9 \cdot 1)\end{array}$ & $\begin{array}{c}8 \cdot 9 \\
(4 \cdot 5-13 \cdot 3)\end{array}$ & $\begin{array}{c}6 \cdot 8 \\
(4 \cdot 9-8 \cdot 7)\end{array}$ &.. \\
\hline \multirow[t]{2}{*}{ 2010-14 } & $\begin{array}{c}13 \cdot 7 \dagger \\
(3 \cdot 8-23 \cdot 6)\end{array}$ & $\begin{array}{l}30 \cdot 0 \\
(22 \cdot 9-37 \cdot 0)\end{array}$ & $\begin{array}{l}55 \cdot 9 \\
(46 \cdot 1-65 \cdot 8)\end{array}$ & $\begin{array}{l}58 \cdot 0 \\
(46 \cdot 6-69 \cdot 4)\end{array}$ & $\begin{array}{c}9.6 \dagger \\
(3.9-15 \cdot 2)\end{array}$ & $\begin{array}{l}19 \cdot 0 \\
(12 \cdot 0-26 \cdot 0)\end{array}$ & $\begin{array}{c}10 \cdot 1 \\
(7 \cdot 1-13 \cdot 2)\end{array}$ & .. \\
\hline & & & & & & & \multicolumn{2}{|c|}{ (Table 6 continues on next page) } \\
\hline
\end{tabular}




\begin{tabular}{|c|c|c|c|c|c|c|c|c|}
\hline & \multicolumn{6}{|c|}{ Gastrointestinal cancers } & \multirow[t]{2}{*}{ Lung } & \multirow{2}{*}{$\begin{array}{l}\text { Melanoma of } \\
\text { the skin }\end{array}$} \\
\hline & Oesophagus & Stomach & Colon & Rectum & Liver & Pancreas & & \\
\hline \multicolumn{9}{|c|}{ (Continued from previous page) } \\
\hline \multicolumn{9}{|l|}{ Mongolia* } \\
\hline 2000-04 &.. &.. &.. &.. &.. &.. &.. &.. \\
\hline 2005-09 & .. & .. & .. & .. & .. & .. & .. & .. \\
\hline 2010-14 &.. &.. &.. &.. &.. &.. &.. &.. \\
\hline \multicolumn{9}{|l|}{ Qatar* } \\
\hline 2000-04 & $\begin{array}{l}36 \cdot 1+5 \\
(10 \cdot 3-61 \cdot 9)\end{array}$ & $\begin{array}{l}15 \cdot 0 \S \\
(2 \cdot 0-28 \cdot 0)\end{array}$ & $\begin{array}{l}62 \cdot 15 \\
(50 \cdot 1-74 \cdot 1)\end{array}$ & $\begin{array}{l}67 \cdot 8+5 \\
(48 \cdot 7-86 \cdot 9)\end{array}$ & $\begin{array}{c}7.15 \\
(2 \cdot 6-11 \cdot 5)\end{array}$ & $\begin{array}{c}9.9+5 \\
(0 \cdot 0-21 \cdot 2)\end{array}$ & $\begin{array}{c}7.4+5 \\
(0.8-14 \cdot 1)\end{array}$ & $\begin{array}{l}77 \cdot 5+5 \\
(48 \cdot 0-100 \cdot 0)\end{array}$ \\
\hline 2005-09 & $\begin{array}{c}33 \cdot 4+5 \\
(9 \cdot 4-57 \cdot 5)\end{array}$ & $\begin{array}{l}22 \cdot 0 S \\
(12 \cdot 2-31 \cdot 7)\end{array}$ & $\begin{array}{l}64 \cdot 35 \\
(47 \cdot 6-80 \cdot 9)\end{array}$ & $\begin{array}{l}60 \cdot 5 \Omega \\
(46 \cdot 7-74 \cdot 3)\end{array}$ & $\begin{array}{c}10 \cdot 3+5 \\
(3 \cdot 2-17 \cdot 3)\end{array}$ & $\begin{array}{c}4 \cdot 2+5 \\
(0 \cdot 0-10 \cdot 4)\end{array}$ & $\begin{array}{c}14 \cdot 35 \\
(7 \cdot 4-21 \cdot 2)\end{array}$ & $\begin{array}{l}30 \cdot 1+5 \\
(0 \cdot 0-65 \cdot 3)\end{array}$ \\
\hline 2010-14 & $\begin{array}{c}42 \cdot 2+5 \\
(9 \cdot 4-75 \cdot 0)\end{array}$ & $\begin{array}{l}17 \cdot 5 \S \\
(9 \cdot 7-25 \cdot 3)\end{array}$ & $\begin{array}{l}63 \cdot 5 \S \\
(51 \cdot 7-75 \cdot 2)\end{array}$ & $\begin{array}{l}43 \cdot 6 \$ \\
(31 \cdot 2-56 \cdot 1)\end{array}$ & $\begin{array}{l}27 \cdot 25 \\
(12 \cdot 9-41 \cdot 5)\end{array}$ & $\begin{array}{l}16 \cdot 6 \$ \\
(7 \cdot 2-26 \cdot 1)\end{array}$ & $\begin{array}{c}17 \cdot 25 \\
(10 \cdot 3-24 \cdot 2)\end{array}$ & $\begin{array}{l}99 \cdot 7+5 \\
(84 \cdot 7-100 \cdot 0)\end{array}$ \\
\hline \multicolumn{9}{|l|}{ Singapore* } \\
\hline 2000-04 & $\begin{array}{c}9.0 \\
(6 \cdot 2-11 \cdot 8)\end{array}$ & $\begin{array}{l}25 \cdot 4 \\
(23 \cdot 3-27 \cdot 4)\end{array}$ & $\begin{array}{l}56 \cdot 1 \\
(54 \cdot 4-57 \cdot 8)\end{array}$ & $\begin{array}{l}51 \cdot 4 \\
(48 \cdot 5-54 \cdot 2)\end{array}$ & $\begin{array}{l}13 \cdot 2 \\
(11 \cdot 6-14 \cdot 9)\end{array}$ & $\begin{array}{c}5 \cdot 3 \\
(3 \cdot 6-7 \cdot 0)\end{array}$ & $\begin{array}{l}10 \cdot 3 \\
(9 \cdot 4-11 \cdot 2)\end{array}$ & $\begin{array}{l}62 \cdot 8 \\
(51 \cdot 0-74 \cdot 6)\end{array}$ \\
\hline 2005-09 & $\begin{array}{c}10 \cdot 5 \\
(7 \cdot 4-13 \cdot 5)\end{array}$ & $\begin{array}{l}27 \cdot 1 \\
(25 \cdot 0-29 \cdot 2)\end{array}$ & $\begin{array}{l}60 \cdot 3 \\
(58 \cdot 7-61 \cdot 8)\end{array}$ & $\begin{array}{l}59 \cdot 0 \\
(56 \cdot 3-61 \cdot 8)\end{array}$ & $\begin{array}{l}20 \cdot 4 \\
(18 \cdot 6-22 \cdot 2)\end{array}$ & $\begin{array}{c}6 \cdot 7 \\
(5 \cdot 1-8 \cdot 3)\end{array}$ & $\begin{array}{l}13 \cdot 0 \\
(12 \cdot 0-13 \cdot 9)\end{array}$ & $\begin{array}{l}59 \cdot 0 \\
(50 \cdot 5-67.5)\end{array}$ \\
\hline 2010-14 & $\begin{array}{l}14 \cdot 8 \\
(11 \cdot 1-18 \cdot 4)\end{array}$ & $\begin{array}{l}30 \cdot 3 \\
(28 \cdot 2-32 \cdot 4)\end{array}$ & $\begin{array}{l}61 \cdot 7 \\
(60 \cdot 2-63 \cdot 2)\end{array}$ & $\begin{array}{l}60 \cdot 5 \\
(58 \cdot 0-63 \cdot 0)\end{array}$ & $\begin{array}{l}24 \cdot 7 \\
(22 \cdot 8-26 \cdot 6)\end{array}$ & $\begin{array}{c}9 \cdot 4 \\
(7 \cdot 6-11 \cdot 2)\end{array}$ & $\begin{array}{l}15 \cdot 5 \\
(14 \cdot 5-16 \cdot 5)\end{array}$ & $\begin{array}{l}60 \cdot 0 \\
(51 \cdot 7-68 \cdot 2)\end{array}$ \\
\hline \multicolumn{9}{|l|}{ Taiwan* } \\
\hline 2000-04 & $\begin{array}{l}13 \cdot 0 \\
(12 \cdot 0-13 \cdot 9)\end{array}$ & $\begin{array}{l}35 \cdot 6 \\
(34 \cdot 8-36 \cdot 4)\end{array}$ & $\begin{array}{l}56 \cdot 8 \\
(56 \cdot 0-57 \cdot 5)\end{array}$ & $\begin{array}{l}57 \cdot 7 \\
(56 \cdot 8-58 \cdot 7)\end{array}$ & $\begin{array}{l}19 \cdot 5 \\
(19 \cdot 1-19 \cdot 9)\end{array}$ & $\begin{array}{c}7 \cdot 0 \\
(6 \cdot 3-7 \cdot 7)\end{array}$ & $\begin{array}{c}11 \cdot 6 \\
(11 \cdot 2-12 \cdot 0)\end{array}$ & $\begin{array}{l}48 \cdot 6 \\
(44 \cdot 6-52 \cdot 6)\end{array}$ \\
\hline 2005-09 & $\begin{array}{l}13 \cdot 2 \\
(12 \cdot 3-14 \cdot 1)\end{array}$ & $\begin{array}{l}36 \cdot 7 \\
(35 \cdot 9-37 \cdot 5)\end{array}$ & $\begin{array}{l}60 \cdot 4 \\
(59 \cdot 8-61 \cdot 0)\end{array}$ & $\begin{array}{l}61 \cdot 5 \\
(60 \cdot 6-62 \cdot 3)\end{array}$ & $\begin{array}{l}24 \cdot 4 \\
(24 \cdot 0-24 \cdot 8)\end{array}$ & $\begin{array}{c}6 \cdot 5 \\
(5 \cdot 9-7 \cdot 1)\end{array}$ & $\begin{array}{l}16 \cdot 0 \\
(15 \cdot 6-16 \cdot 4)\end{array}$ & $\begin{array}{l}53 \cdot 3 \\
(49 \cdot 7-56 \cdot 9)\end{array}$ \\
\hline 2010-14 & $\begin{array}{l}15 \cdot 5 \\
(14 \cdot 6-16 \cdot 4)\end{array}$ & $\begin{array}{l}38 \cdot 6 \\
(37 \cdot 8-39 \cdot 4)\end{array}$ & $\begin{array}{l}62 \cdot 6 \\
(62 \cdot 0-63 \cdot 1)\end{array}$ & $\begin{array}{l}62 \cdot 5 \\
(61 \cdot 7-63 \cdot 3)\end{array}$ & $\begin{array}{l}27 \cdot 9 \\
(27 \cdot 5-28 \cdot 4)\end{array}$ & $\begin{array}{c}7 \cdot 7 \\
(7 \cdot 0-8 \cdot 3)\end{array}$ & $\begin{array}{l}20 \cdot 6 \\
(20 \cdot 2-21 \cdot 0)\end{array}$ & $\begin{array}{l}52 \cdot 1 \\
(48 \cdot 5-55 \cdot 6)\end{array}$ \\
\hline \multicolumn{9}{|c|}{ Thailand (six registries) } \\
\hline 2000-04 & $\begin{array}{c}8.85 \\
(6 \cdot 6-11 \cdot 1)\end{array}$ & $\begin{array}{l}22 \cdot 38 \\
(18 \cdot 7-25 \cdot 8)\end{array}$ & $\begin{array}{l}39 \cdot 85 \\
(37 \cdot 2-42 \cdot 5)\end{array}$ & $\begin{array}{l}43 \cdot 05 \\
(38 \cdot 5-47 \cdot 4)\end{array}$ & $\begin{array}{c}7 \cdot 75 \\
(6 \cdot 9-8 \cdot 6)\end{array}$ & $\begin{array}{c}6.98 \\
(5 \cdot 1-8 \cdot 7)\end{array}$ & $\begin{array}{l}10 \cdot 25 \\
(9 \cdot 3-11 \cdot 1)\end{array}$ & $\begin{array}{l}44 \cdot 75 \\
(34 \cdot 4-55 \cdot 0)\end{array}$ \\
\hline 2005-09 & $\begin{array}{c}7.8 \\
(5.9-9.7)\end{array}$ & $\begin{array}{l}16 \cdot 1 \\
(13 \cdot 9-18 \cdot 3)\end{array}$ & $\begin{array}{l}42 \cdot 8 \\
(41 \cdot 1-44 \cdot 6)\end{array}$ & $\begin{array}{l}38 \cdot 6 \\
(36 \cdot 0-41 \cdot 1)\end{array}$ & $\begin{array}{c}7.9 \\
(7 \cdot 3-8 \cdot 6)\end{array}$ & $\begin{array}{c}9.6 \\
(7 \cdot 7-11 \cdot 5)\end{array}$ & $\begin{array}{l}10 \cdot 3 \\
(9 \cdot 6-11 \cdot 1)\end{array}$ & $\begin{array}{l}37 \cdot 9 \Omega \\
(30 \cdot 6-45 \cdot 2)\end{array}$ \\
\hline 2010-14 & $\begin{array}{c}7 \cdot 1 \\
(5 \cdot 1-9 \cdot 1)\end{array}$ & $\begin{array}{l}12 \cdot 5 \\
(10 \cdot 7-14 \cdot 3)\end{array}$ & $\begin{array}{l}47 \cdot 0 \\
(45 \cdot 2-48 \cdot 8)\end{array}$ & $\begin{array}{l}44 \cdot 4 \\
(41 \cdot 8-47 \cdot 1)\end{array}$ & $\begin{array}{c}6 \cdot 9 \\
(6 \cdot 3-7 \cdot 5)\end{array}$ & $\begin{array}{c}6 \cdot 8 \\
(5 \cdot 3-8 \cdot 2)\end{array}$ & $\begin{array}{c}8 \cdot 6 \\
(7 \cdot 9-9 \cdot 2)\end{array}$ & $\begin{array}{l}29 \cdot 95 \\
(23 \cdot 7-36 \cdot 1)\end{array}$ \\
\hline \multicolumn{9}{|c|}{ Turkey (nine registries) } \\
\hline 2000-04 & $\begin{array}{l}14 \cdot 85 \\
(9 \cdot 2-20 \cdot 4)\end{array}$ & $\begin{array}{l}21 \cdot 9 \sqrt{ } \\
(18 \cdot 5-25 \cdot 4)\end{array}$ & $\begin{array}{l}52 \cdot 98 \\
(49 \cdot 1-56 \cdot 7)\end{array}$ & $\begin{array}{l}48 \cdot 5 \\
(43 \cdot 9-53 \cdot 0)\end{array}$ & $\begin{array}{l}20 \cdot 15 \\
(15 \cdot 0-25 \cdot 2)\end{array}$ & $\begin{array}{l}10 \cdot 9 \sqrt{ } \\
(7 \cdot 8-14 \cdot 1)\end{array}$ & $\begin{array}{l}11 \cdot 9 \Omega \\
(10 \cdot 6-13 \cdot 3)\end{array}$ & $\begin{array}{l}60.95 \\
(53 \cdot 2-68.6)\end{array}$ \\
\hline 2005-09 & $\begin{array}{l}14 \cdot 7 \\
(12 \cdot 9-16 \cdot 5)\end{array}$ & $\begin{array}{l}21 \cdot 4 \\
(20 \cdot 3-22 \cdot 4)\end{array}$ & $\begin{array}{l}52 \cdot 7 \\
(51 \cdot 3-54 \cdot 2)\end{array}$ & $\begin{array}{l}49 \cdot 3 \\
(47 \cdot 3-51 \cdot 3)\end{array}$ & $\begin{array}{l}14 \cdot 9 \\
(12 \cdot 9-17 \cdot 0)\end{array}$ & $\begin{array}{c}9.5 \\
(8 \cdot 4-10 \cdot 7)\end{array}$ & $\begin{array}{l}12 \cdot 7 \\
(12 \cdot 2-13 \cdot 2)\end{array}$ & $\begin{array}{l}57 \cdot 7 \\
(54 \cdot 6-60 \cdot 9)\end{array}$ \\
\hline 2010-14 & $\begin{array}{l}19 \cdot 0 \\
(16 \cdot 9-21 \cdot 1)\end{array}$ & $\begin{array}{l}24 \cdot 6 \\
(23 \cdot 6-25 \cdot 6)\end{array}$ & $\begin{array}{l}55 \cdot 2 \\
(53 \cdot 8-56 \cdot 6)\end{array}$ & $\begin{array}{l}52 \cdot 6 \\
(50 \cdot 6-54 \cdot 5)\end{array}$ & $\begin{array}{l}15 \cdot 9 \\
(14 \cdot 0-17 \cdot 8)\end{array}$ & $\begin{array}{l}10 \cdot 4 \\
(9 \cdot 3-11 \cdot 5)\end{array}$ & $\begin{array}{l}14 \cdot 9 \\
(14 \cdot 3-15 \cdot 4)\end{array}$ & $\begin{array}{l}60 \cdot 7 \\
(57 \cdot 6-63 \cdot 7)\end{array}$ \\
\hline \multicolumn{9}{|l|}{ Europe } \\
\hline \multicolumn{9}{|l|}{ Austria* } \\
\hline $2000-04$ & $\begin{array}{l}16 \cdot 2 \\
(14 \cdot 2-18 \cdot 3)\end{array}$ & $\begin{array}{l}30 \cdot 0 \\
(28 \cdot 7-31 \cdot 3)\end{array}$ & $\begin{array}{l}60 \cdot 7 \\
(59 \cdot 7-61 \cdot 7)\end{array}$ & $\begin{array}{l}60 \cdot 2 \\
(58 \cdot 9-61 \cdot 5)\end{array}$ & $\begin{array}{l}11 \cdot 2 \\
(10 \cdot 0-12 \cdot 4)\end{array}$ & $\begin{array}{c}6.75 \\
(5 \cdot 9-7 \cdot 5)\end{array}$ & $\begin{array}{l}15 \cdot 4 \\
(14 \cdot 8-16 \cdot 0)\end{array}$ & $\begin{array}{l}83 \cdot 4 \\
(82 \cdot 2-84 \cdot 7)\end{array}$ \\
\hline 2005-09 & $\begin{array}{l}16 \cdot 8 \\
(15 \cdot 0-18 \cdot 7)\end{array}$ & $\begin{array}{l}34 \cdot 2 \\
(32 \cdot 9-35 \cdot 6)\end{array}$ & $\begin{array}{l}63 \cdot 5 \\
(62 \cdot 5-64 \cdot 4)\end{array}$ & $\begin{array}{l}63 \cdot 5 \\
(62 \cdot 2-64 \cdot 8)\end{array}$ & $\begin{array}{l}14 \cdot 2 \Omega \\
(12 \cdot 9-15 \cdot 6)\end{array}$ & $\begin{array}{c}8.5 \\
(7 \cdot 7-9 \cdot 4)\end{array}$ & $\begin{array}{l}18 \cdot 0 \\
(17 \cdot 4-18 \cdot 6)\end{array}$ & $\begin{array}{l}84 \cdot 6 \\
(83 \cdot 5-85 \cdot 8)\end{array}$ \\
\hline 2010-14 & $\begin{array}{l}18 \cdot 6 \\
(16 \cdot 6-20 \cdot 6)\end{array}$ & $\begin{array}{l}35 \cdot 4 \\
(34 \cdot 0-36 \cdot 9)\end{array}$ & $\begin{array}{l}63 \cdot 7 \\
(62 \cdot 7-64 \cdot 7)\end{array}$ & $\begin{array}{l}64 \cdot 2 \\
(62 \cdot 9-65 \cdot 6)\end{array}$ & $\begin{array}{l}14 \cdot 85 \\
(13 \cdot 4-16 \cdot 2)\end{array}$ & $\begin{array}{l}10 \cdot 5 \S \\
(9 \cdot 5-11 \cdot 4)\end{array}$ & $\begin{array}{l}19 \cdot 7 \\
(19 \cdot 1-20 \cdot 4)\end{array}$ & $\begin{array}{l}87 \cdot 8 \\
(86 \cdot 7-88 \cdot 8)\end{array}$ \\
\hline \multicolumn{9}{|l|}{ Belgium* } \\
\hline 2000-04 & $\begin{array}{l}16 \cdot 6 \\
(13 \cdot 8-19 \cdot 4)\end{array}$ & $\begin{array}{l}29 \cdot 3 \\
(26 \cdot 4-32 \cdot 2)\end{array}$ & $\begin{array}{l}64 \cdot 3 \\
(62 \cdot 7-66 \cdot 0)\end{array}$ & $\begin{array}{l}62 \cdot 9 \\
(60 \cdot 4-65 \cdot 4)\end{array}$ & $\begin{array}{l}21 \cdot 3 \\
(16 \cdot 8-25 \cdot 8)\end{array}$ & $\begin{array}{c}8.9 \\
(6 \cdot 9-10 \cdot 8)\end{array}$ & $\begin{array}{l}15 \cdot 4 \\
(14 \cdot 4-16 \cdot 4)\end{array}$ & $\begin{array}{l}86 \cdot 3 \\
(83 \cdot 9-88 \cdot 7)\end{array}$ \\
\hline 2005-09 & $\begin{array}{l}23 \cdot 2 \\
(21 \cdot 7-24 \cdot 7)\end{array}$ & $\begin{array}{l}35 \cdot 8 \\
(34 \cdot 4-37 \cdot 1)\end{array}$ & $\begin{array}{l}65 \cdot 0 \\
(64 \cdot 3-65 \cdot 7)\end{array}$ & $\begin{array}{l}65 \cdot 3 \\
(64 \cdot 3-66 \cdot 4)\end{array}$ & $\begin{array}{l}20 \cdot 6 \\
(19 \cdot 0-22 \cdot 3)\end{array}$ & $\begin{array}{l}10 \cdot 5 \\
(9 \cdot 6-11 \cdot 4)\end{array}$ & $\begin{array}{l}17 \cdot 0 \\
(16 \cdot 5-17 \cdot 4)\end{array}$ & $\begin{array}{l}88.9 \\
(87 \cdot 9-89 \cdot 8)\end{array}$ \\
\hline \multirow[t]{2}{*}{ 2010-14 } & $\begin{array}{l}23 \cdot 6 \\
(22 \cdot 1-25 \cdot 0)\end{array}$ & $\begin{array}{l}37 \cdot 5 \\
(36 \cdot 2-38 \cdot 9)\end{array}$ & $\begin{array}{l}67 \cdot 9 \\
(67 \cdot 2-68 \cdot 6)\end{array}$ & $\begin{array}{l}66 \cdot 6 \\
(65 \cdot 6-67 \cdot 6)\end{array}$ & $\begin{array}{l}20 \cdot 7 \\
(19 \cdot 2-22 \cdot 2)\end{array}$ & $\begin{array}{l}12 \cdot 4 \\
(11 \cdot 4-13 \cdot 3)\end{array}$ & $\begin{array}{l}18 \cdot 2 \\
(17 \cdot 7-18 \cdot 6)\end{array}$ & $\begin{array}{l}91 \cdot 0 \\
(90 \cdot 1-91 \cdot 8)\end{array}$ \\
\hline & & & & & & & \multicolumn{2}{|c|}{ (Table 6 continues on next page } \\
\hline
\end{tabular}




\begin{tabular}{|c|c|c|c|c|c|c|c|c|}
\hline & \multicolumn{6}{|c|}{ Gastrointestinal cancers } & \multirow[t]{2}{*}{ Lung } & \multirow{2}{*}{$\begin{array}{l}\text { Melanoma of } \\
\text { the skin }\end{array}$} \\
\hline & Oesophagus & Stomach & Colon & Rectum & Liver & Pancreas & & \\
\hline \multicolumn{9}{|c|}{ (Continued from previous page) } \\
\hline \multicolumn{9}{|l|}{ Bulgaria* } \\
\hline 2000-04 & .. & $\begin{array}{l}11 \cdot 25 \\
(10 \cdot 3-12 \cdot 0)\end{array}$ & $\begin{array}{l}43 \cdot 9 \\
(42 \cdot 7-45 \cdot 2)\end{array}$ & $\begin{array}{l}37 \cdot 0 \\
(35 \cdot 6-38 \cdot 4)\end{array}$ & $\begin{array}{c}4 \cdot 05 \\
(2 \cdot 9-5 \cdot 1)\end{array}$ & .. & $\begin{array}{c}5 \cdot 8 \\
(5 \cdot 3-6 \cdot 3)\end{array}$ & $\begin{array}{l}50 \cdot 8 \\
(47 \cdot 9-53 \cdot 8)\end{array}$ \\
\hline 2005-09 & .. & $\begin{array}{l}13 \cdot 9 \\
(13 \cdot 0-14 \cdot 9)\end{array}$ & $\begin{array}{l}48 \cdot 5 \\
(47 \cdot 4-49 \cdot 6)\end{array}$ & $\begin{array}{l}42 \cdot 1 \\
(40 \cdot 7-43 \cdot 5)\end{array}$ & $\begin{array}{c}4 \cdot 65 \\
(3 \cdot 5-5 \cdot 6)\end{array}$ &.$\cdot$ & $\begin{array}{c}7 \cdot 0 \\
(6 \cdot 5-7 \cdot 6)\end{array}$ & $\begin{array}{l}56 \cdot 0 \\
(53 \cdot 3-58 \cdot 6)\end{array}$ \\
\hline 2010-14 &. & $\begin{array}{l}16 \cdot 0 \\
(14 \cdot 9-17 \cdot 0)\end{array}$ & $\begin{array}{l}52 \cdot 4 \\
(51 \cdot 3-53 \cdot 6)\end{array}$ & $\begin{array}{l}45 \cdot 9 \\
(44 \cdot 4-47 \cdot 4)\end{array}$ & $\begin{array}{c}6 \cdot 55 \\
(5 \cdot 1-7 \cdot 9)\end{array}$ & .. & $\begin{array}{c}7 \cdot 7 \\
(7 \cdot 1-8 \cdot 2)\end{array}$ & $\begin{array}{l}61 \cdot 2 \\
(58 \cdot 8-63 \cdot 7)\end{array}$ \\
\hline \multicolumn{9}{|l|}{ Croatia* } \\
\hline $2000-04$ & $\begin{array}{c}6.8 \\
(4.9-8 \cdot 7)\end{array}$ & $\begin{array}{l}19 \cdot 7 \\
(18 \cdot 4-20 \cdot 9)\end{array}$ & $\begin{array}{l}47 \cdot 3 \\
(45 \cdot 9-48 \cdot 7)\end{array}$ & $\begin{array}{l}44 \cdot 4 \\
(42 \cdot 7-46 \cdot 1)\end{array}$ & $\begin{array}{c}9 \cdot 25 \\
(7 \cdot 6-10 \cdot 7)\end{array}$ & $\begin{array}{c}9 \cdot 1 \\
(7 \cdot 9-10 \cdot 4)\end{array}$ & $\begin{array}{l}11 \cdot 2 \\
(10 \cdot 5-11 \cdot 9)\end{array}$ & $\begin{array}{l}66 \cdot 4 \\
(64 \cdot 0-68 \cdot 8)\end{array}$ \\
\hline 2005-09 & $\begin{array}{c}9 \cdot 1 \\
(6 \cdot 9-11 \cdot 2)\end{array}$ & $\begin{array}{l}18 \cdot 9 \\
(17 \cdot 6-20 \cdot 2)\end{array}$ & $\begin{array}{l}49 \cdot 5 \\
(48 \cdot 2-50 \cdot 8)\end{array}$ & $\begin{array}{l}47 \cdot 1 \\
(45 \cdot 5-48 \cdot 7)\end{array}$ & $\begin{array}{c}9 \cdot 25 \\
(7 \cdot 7-10 \cdot 8)\end{array}$ & $\begin{array}{c}8 \cdot 2 \\
(7 \cdot 0-9 \cdot 4)\end{array}$ & $\begin{array}{l}10 \cdot 6 \\
(10 \cdot 0-11 \cdot 3)\end{array}$ & $\begin{array}{l}74 \cdot 7 \\
(72 \cdot 7-76 \cdot 7)\end{array}$ \\
\hline $2010-14$ & $\begin{array}{c}8.7 \\
(6 \cdot 6-10 \cdot 8)\end{array}$ & $\begin{array}{l}20 \cdot 0 \\
(18 \cdot 6-21 \cdot 3)\end{array}$ & $\begin{array}{c}51 \cdot 1 \\
(49 \cdot 9-52 \cdot 4)\end{array}$ & $\begin{array}{l}48 \cdot 2 \\
(46 \cdot 5-49 \cdot 8)\end{array}$ & $\begin{array}{c}9.35 \\
(7 \cdot 7-10 \cdot 9)\end{array}$ & $\begin{array}{c}8 \cdot 45 \\
(7 \cdot 1-9 \cdot 7)\end{array}$ & $\begin{array}{l}10 \cdot 0 \\
(9 \cdot 3-10 \cdot 6)\end{array}$ & $\begin{array}{l}77 \cdot 2 \\
(75 \cdot 3-79 \cdot 1)\end{array}$ \\
\hline \multicolumn{9}{|c|}{ Czech Republic* } \\
\hline 2000-04 & $\begin{array}{c}7 \cdot 3 \\
(6 \cdot 0-8 \cdot 6)\end{array}$ & $\begin{array}{l}18 \cdot 7 \\
(17 \cdot 7-19 \cdot 7)\end{array}$ & $\begin{array}{l}48 \cdot 0 \\
(47 \cdot 3-48 \cdot 8)\end{array}$ & $\begin{array}{l}43.8 \\
(42 \cdot 6-44.9)\end{array}$ & $\begin{array}{c}3 \cdot 0 \\
(2 \cdot 3-3 \cdot 6)\end{array}$ & $\begin{array}{c}3 \cdot 6 \\
(3 \cdot 0-4 \cdot 1)\end{array}$ & $\begin{array}{c}8 \cdot 6 \\
(8 \cdot 2-9 \cdot 0)\end{array}$ & $\begin{array}{l}80 \cdot 7 \\
(79 \cdot 5-81 \cdot 8)\end{array}$ \\
\hline 2005-09 & $\begin{array}{c}9 \cdot 0 \\
(7 \cdot 5-10 \cdot 5)\end{array}$ & $\begin{array}{l}19 \cdot 3 \\
(18 \cdot 3-20 \cdot 3)\end{array}$ & $\begin{array}{l}52 \cdot 0 \\
(51 \cdot 3-52 \cdot 7)\end{array}$ & $\begin{array}{l}47 \cdot 9 \\
(46 \cdot 8-49 \cdot 0)\end{array}$ & $\begin{array}{c}5 \cdot 3 \\
(4 \cdot 5-6 \cdot 2)\end{array}$ & $\begin{array}{c}4 \cdot 7 \\
(4 \cdot 1-5 \cdot 3)\end{array}$ & $\begin{array}{c}9 \cdot 8 \\
(9 \cdot 4-10 \cdot 3)\end{array}$ & $\begin{array}{l}84 \cdot 6 \\
(83 \cdot 7-85 \cdot 6)\end{array}$ \\
\hline 2010-14 & $\begin{array}{c}9.8 \\
(8 \cdot 3-11 \cdot 4)\end{array}$ & $\begin{array}{l}20 \cdot 6 \\
(19 \cdot 6-21 \cdot 6)\end{array}$ & $\begin{array}{l}56 \cdot 1 \\
(55 \cdot 4-56 \cdot 9)\end{array}$ & $\begin{array}{l}52 \cdot 3 \\
(51 \cdot 2-53 \cdot 4)\end{array}$ & $\begin{array}{c}6 \cdot 7 \\
(5 \cdot 6-7 \cdot 7)\end{array}$ & $\begin{array}{c}6 \cdot 1 \\
(5 \cdot 4-6 \cdot 7)\end{array}$ & $\begin{array}{l}10 \cdot 6 \\
(10 \cdot 2-11 \cdot 1)\end{array}$ & $\begin{array}{l}85 \cdot 7 \\
(84 \cdot 8-86 \cdot 6)\end{array}$ \\
\hline \multicolumn{9}{|l|}{ Denmark* } \\
\hline 2000-04 & $\begin{array}{c}8 \cdot 4 \\
(6 \cdot 9-9 \cdot 8)\end{array}$ & $\begin{array}{l}14 \cdot 7 \\
(13 \cdot 2-16 \cdot 3)\end{array}$ & $\begin{array}{l}51 \cdot 5 \\
(50 \cdot 4-52 \cdot 6)\end{array}$ & $\begin{array}{l}53 \cdot 2 \\
(51 \cdot 7-54 \cdot 7)\end{array}$ & $\begin{array}{c}4 \cdot 4 \\
(3 \cdot 1-5 \cdot 8)\end{array}$ & $\begin{array}{c}3 \cdot 8 \\
(3 \cdot 0-4 \cdot 5)\end{array}$ & $\begin{array}{c}9.5 \\
(9 \cdot 0-10 \cdot 1)\end{array}$ & $\begin{array}{l}87 \cdot 3 \\
(86 \cdot 0-88 \cdot 5)\end{array}$ \\
\hline $2005-09$ & $\begin{array}{l}10 \cdot 4 \\
(8 \cdot 9-11 \cdot 9)\end{array}$ & $\begin{array}{l}15 \cdot 4 \\
(13 \cdot 9-16 \cdot 9)\end{array}$ & $\begin{array}{l}56 \cdot 5 \\
(55 \cdot 5-57 \cdot 6)\end{array}$ & $\begin{array}{l}59.8 \\
(58 \cdot 4-61 \cdot 1)\end{array}$ & $\begin{array}{c}5 \cdot 6 \\
(4 \cdot 2-7 \cdot 0)\end{array}$ & $\begin{array}{c}5 \cdot 6 \\
(4 \cdot 7-6 \cdot 4)\end{array}$ & $\begin{array}{l}12 \cdot 3 \\
(11 \cdot 7-12 \cdot 8)\end{array}$ & $\begin{array}{l}89 \cdot 1 \\
(88 \cdot 1-90 \cdot 0)\end{array}$ \\
\hline 2010-14 & $\begin{array}{l}13 \cdot 7 \\
(12 \cdot 0-15 \cdot 4)\end{array}$ & $\begin{array}{l}19 \cdot 9 \\
(18 \cdot 1-21 \cdot 6)\end{array}$ & $\begin{array}{l}61 \cdot 6 \\
(60 \cdot 6-62 \cdot 7)\end{array}$ & $\begin{array}{l}64 \cdot 8 \\
(63 \cdot 4-66 \cdot 1)\end{array}$ & $\begin{array}{c}7 \cdot 5 \\
(5 \cdot 9-9 \cdot 1)\end{array}$ & $\begin{array}{c}8 \cdot 0 \\
(7 \cdot 0-9 \cdot 1)\end{array}$ & $\begin{array}{l}16 \cdot 6 \\
(16 \cdot 0-17 \cdot 3)\end{array}$ & $\begin{array}{l}91 \cdot 1 \\
(90 \cdot 2-91 \cdot 9)\end{array}$ \\
\hline \multicolumn{9}{|l|}{ Estonia* } \\
\hline 2000-04 & $\begin{array}{c}5 \cdot 7 \\
(2 \cdot 8-8 \cdot 6)\end{array}$ & $\begin{array}{l}22 \cdot 3 \\
(20 \cdot 2-24 \cdot 4)\end{array}$ & $\begin{array}{l}48 \cdot 9 \\
(46 \cdot 2-51 \cdot 5)\end{array}$ & $\begin{array}{l}46 \cdot 4 \\
(42 \cdot 9-49 \cdot 9)\end{array}$ & $\begin{array}{c}5 \cdot 5 \S \\
(2 \cdot 8-8 \cdot 3)\end{array}$ & $\begin{array}{c}4 \cdot 8 \\
(3 \cdot 2-6 \cdot 5)\end{array}$ & $\begin{array}{l}10 \cdot 8 \\
(9 \cdot 5-12 \cdot 2)\end{array}$ & $\begin{array}{l}71 \cdot 4 \\
(67 \cdot 0-75 \cdot 8)\end{array}$ \\
\hline 2005-09 & $\begin{array}{c}6 \cdot 0 \\
(3 \cdot 5-8 \cdot 5)\end{array}$ & $\begin{array}{l}24 \cdot 2 \\
(22 \cdot 1-26 \cdot 4)\end{array}$ & $\begin{array}{l}53 \cdot 8 \\
(51 \cdot 3-56 \cdot 3)\end{array}$ & $\begin{array}{l}50 \cdot 5 \\
(46.9-54 \cdot 1)\end{array}$ & $\begin{array}{c}6 \cdot 5 \\
(3 \cdot 9-9 \cdot 2)\end{array}$ & $\begin{array}{c}5 \cdot 4 \\
(3 \cdot 8-7 \cdot 0)\end{array}$ & $\begin{array}{l}14 \cdot 3 \\
(12 \cdot 6-15 \cdot 9)\end{array}$ & $\begin{array}{l}75 \cdot 2 \\
(71 \cdot 7-78 \cdot 8)\end{array}$ \\
\hline $2010-14$ & $\begin{array}{c}5 \cdot 4 \\
(2 \cdot 3-8 \cdot 5)\end{array}$ & $\begin{array}{l}29 \cdot 2 \\
(26 \cdot 1-32 \cdot 3)\end{array}$ & $\begin{array}{l}58 \cdot 4 \\
(55 \cdot 2-61 \cdot 6)\end{array}$ & $\begin{array}{l}54 \cdot 8 \\
(50 \cdot 1-59 \cdot 5)\end{array}$ & $\begin{array}{c}4 \cdot 2 \\
(1 \cdot 8-6 \cdot 6)\end{array}$ & $\begin{array}{l}10 \cdot 2 \\
(7 \cdot 0-13 \cdot 4)\end{array}$ & $\begin{array}{l}16 \cdot 9 \\
(14 \cdot 9-19 \cdot 0)\end{array}$ & $\begin{array}{l}81 \cdot 8 \\
(77 \cdot 6-86 \cdot 0)\end{array}$ \\
\hline \multicolumn{9}{|l|}{ Finland* } \\
\hline 2000-04 & $\begin{array}{l}11 \cdot 8 \\
(9 \cdot 5-14 \cdot 1)\end{array}$ & $\begin{array}{l}26 \cdot 0 \\
(24 \cdot 3-27 \cdot 6)\end{array}$ & $\begin{array}{l}61 \cdot 3 \\
(59 \cdot 9-62 \cdot 8)\end{array}$ & $\begin{array}{l}59 \cdot 9 \\
(58 \cdot 0-61 \cdot 9)\end{array}$ & $\begin{array}{c}7 \cdot 1 \\
(5 \cdot 5-8 \cdot 8)\end{array}$ & $\begin{array}{c}4 \cdot 1 \\
(3 \cdot 2-4 \cdot 9)\end{array}$ & $\begin{array}{l}11 \cdot 9 \\
(11 \cdot 0-12 \cdot 7)\end{array}$ & $\begin{array}{l}84 \cdot 8 \\
(83 \cdot 3-86 \cdot 4)\end{array}$ \\
\hline 2005-09 & $\begin{array}{l}12 \cdot 8 \\
(10 \cdot 6-15 \cdot 0)\end{array}$ & $\begin{array}{l}25 \cdot 1 \\
(23 \cdot 4-26 \cdot 8)\end{array}$ & $\begin{array}{l}63 \cdot 2 \\
(61 \cdot 9-64 \cdot 5)\end{array}$ & $\begin{array}{l}63 \cdot 8 \\
(62 \cdot 0-65 \cdot 6)\end{array}$ & $\begin{array}{c}9.15 \\
(7 \cdot 3-10 \cdot 8)\end{array}$ & $\begin{array}{c}7 \cdot 3 \\
(6 \cdot 2-8 \cdot 5)\end{array}$ & $\begin{array}{l}12 \cdot 1 \\
(11 \cdot 3-13 \cdot 0)\end{array}$ & $\begin{array}{l}87 \cdot 4 \\
(86 \cdot 2-88 \cdot 6)\end{array}$ \\
\hline $2010-14$ & $\begin{array}{l}12 \cdot 4 \\
(10 \cdot 5-14 \cdot 4)\end{array}$ & $\begin{array}{l}25 \cdot 7 \\
(23 \cdot 9-27 \cdot 5)\end{array}$ & $\begin{array}{l}64 \cdot 9 \\
(63 \cdot 7-66 \cdot 2)\end{array}$ & $\begin{array}{l}64 \cdot 4 \\
(62 \cdot 6-66 \cdot 1)\end{array}$ & $\begin{array}{l}10 \cdot 45 \\
(8 \cdot 5-12 \cdot 2)\end{array}$ & $\begin{array}{c}7 \cdot 45 \\
(6 \cdot 3-8 \cdot 4)\end{array}$ & $\begin{array}{l}13 \cdot 0 \\
(12 \cdot 1-13 \cdot 9)\end{array}$ & $\begin{array}{l}88 \cdot 7 \\
(87 \cdot 6-89 \cdot 8)\end{array}$ \\
\hline \multicolumn{9}{|c|}{ France (23 registries) $\ddagger$} \\
\hline 2000-04 & $\begin{array}{l}13 \cdot 0 \\
(11 \cdot 8-14 \cdot 2)\end{array}$ & $\begin{array}{l}26 \cdot 3 \\
(24 \cdot 9-27 \cdot 7)\end{array}$ & $\begin{array}{l}60 \cdot 7 \\
(59 \cdot 8-61 \cdot 5)\end{array}$ & $\begin{array}{l}58 \cdot 3 \\
(56 \cdot 9-59 \cdot 6)\end{array}$ & $\begin{array}{l}14 \cdot 0 \\
(12 \cdot 9-15 \cdot 1)\end{array}$ & $\begin{array}{c}7 \cdot 8 \\
(6 \cdot 9-8 \cdot 7)\end{array}$ & $\begin{array}{l}14 \cdot 1 \\
(13 \cdot 5-14 \cdot 6)\end{array}$ & $\begin{array}{l}89 \cdot 7 \\
(88 \cdot 5-90 \cdot 9)\end{array}$ \\
\hline 2005-09 & $\begin{array}{l}14 \cdot 9 \\
(13 \cdot 6-16 \cdot 1)\end{array}$ & $\begin{array}{l}27 \cdot 1 \\
(25 \cdot 8-28 \cdot 4)\end{array}$ & $\begin{array}{l}63 \cdot 6 \\
(62 \cdot 8-64 \cdot 3)\end{array}$ & $\begin{array}{l}60 \cdot 7 \\
(59 \cdot 5-61 \cdot 9)\end{array}$ & $\begin{array}{l}16 \cdot 5 \\
(15 \cdot 4-17 \cdot 6)\end{array}$ & $\begin{array}{c}9 \cdot 0 \\
(8 \cdot 2-9 \cdot 8)\end{array}$ & $\begin{array}{l}16 \cdot 2 \\
(15 \cdot 7-16 \cdot 7)\end{array}$ & $\begin{array}{l}90 \cdot 9 \\
(89 \cdot 9-91 \cdot 9)\end{array}$ \\
\hline \multirow[t]{2}{*}{ 2010-14 } & $\begin{array}{l}13 \cdot 9 \\
(11 \cdot 4-16 \cdot 4)\end{array}$ & $\begin{array}{l}26 \cdot 7 \\
(23 \cdot 9-29 \cdot 6)\end{array}$ & $\begin{array}{l}63 \cdot 7 \\
(62 \cdot 2-65 \cdot 3)\end{array}$ & $\begin{array}{l}60 \cdot 9 \\
(58 \cdot 4-63 \cdot 4)\end{array}$ & $\begin{array}{l}18 \cdot 3 \\
(16 \cdot 0-20 \cdot 5)\end{array}$ & $\begin{array}{c}8 \cdot 6 \\
(7 \cdot 0-10 \cdot 2)\end{array}$ & $\begin{array}{l}17 \cdot 3 \\
(16 \cdot 1-18 \cdot 5)\end{array}$ & $\begin{array}{l}90 \cdot 8 \\
(88 \cdot 7-92 \cdot 9)\end{array}$ \\
\hline & & & & & & & \multicolumn{2}{|c|}{ (Table 6 continues on next page } \\
\hline
\end{tabular}




\begin{tabular}{|c|c|c|c|c|c|c|c|c|}
\hline & \multicolumn{6}{|c|}{ Gastrointestinal cancers } & \multirow[t]{2}{*}{ Lung } & \multirow{2}{*}{$\begin{array}{l}\text { Melanoma of } \\
\text { the skin }\end{array}$} \\
\hline & Oesophagus & Stomach & Colon & Rectum & Liver & Pancreas & & \\
\hline \multicolumn{9}{|c|}{ (Continued from previous page) } \\
\hline \multicolumn{9}{|c|}{ Germany (ten registries) } \\
\hline 2000-04 & $\begin{array}{l}16 \cdot 6 \\
(15 \cdot 3-17 \cdot 8)\end{array}$ & $\begin{array}{l}31 \cdot 8 \\
(30 \cdot 0-33 \cdot 5)\end{array}$ & $\begin{array}{l}62 \cdot 0 \\
(61 \cdot 2-62 \cdot 8)\end{array}$ & $\begin{array}{l}60 \cdot 9 \\
(60 \cdot 2-61 \cdot 6)\end{array}$ & $\begin{array}{l}12 \cdot 5 \S \\
(11 \cdot 6-13 \cdot 4)\end{array}$ & $\begin{array}{c}8.05 \\
(7 \cdot 5-8.6)\end{array}$ & $\begin{array}{l}14 \cdot 9 \\
(14 \cdot 3-15 \cdot 4)\end{array}$ & $\begin{array}{l}91 \cdot 0 \\
(90 \cdot 4-91 \cdot 6)\end{array}$ \\
\hline 2005-09 & $\begin{array}{l}19 \cdot 7 \\
(18 \cdot 8-20 \cdot 6)\end{array}$ & $\begin{array}{c}31 \cdot 4 \\
(30 \cdot 8-32 \cdot 0)\end{array}$ & $\begin{array}{l}64 \cdot 9 \\
(64 \cdot 4-65 \cdot 3)\end{array}$ & $\begin{array}{l}62 \cdot 2 \\
(61 \cdot 6-62 \cdot 8)\end{array}$ & $\begin{array}{l}12 \cdot 9 \\
(11 \cdot 3-14 \cdot 5)\end{array}$ & $\begin{array}{c}9 \cdot 3 \\
(8 \cdot 4-10 \cdot 2)\end{array}$ & $\begin{array}{l}16 \cdot 9 \\
(16 \cdot 5-17 \cdot 4)\end{array}$ & $\begin{array}{l}92.0 \\
(91 \cdot 5-92 \cdot 6)\end{array}$ \\
\hline 2010-14 & $\begin{array}{l}20 \cdot 8 \\
(19 \cdot 8-21 \cdot 8)\end{array}$ & $\begin{array}{l}33 \cdot 5 \\
(32 \cdot 8-34 \cdot 2)\end{array}$ & $\begin{array}{l}64 \cdot 8 \\
(64 \cdot 3-65 \cdot 3)\end{array}$ & $\begin{array}{l}62 \cdot 3 \\
(61 \cdot 6-62 \cdot 9)\end{array}$ & $\begin{array}{l}13 \cdot 0 \\
(10 \cdot 5-15 \cdot 5)\end{array}$ & $\begin{array}{l}10 \cdot 7 \\
(9 \cdot 6-11 \cdot 8)\end{array}$ & $\begin{array}{l}18 \cdot 3 \\
(17 \cdot 9-18 \cdot 8)\end{array}$ & $\begin{array}{l}93 \cdot 1 \\
(92 \cdot 6-93 \cdot 6)\end{array}$ \\
\hline \multicolumn{9}{|l|}{ Gibraltar* } \\
\hline $2000-04$ &.$\cdot$ & $\begin{array}{c}8.0 \dagger \\
(0.0-19.9)\end{array}$ & $\begin{array}{l}38.8 \dagger \\
(17 \cdot 7-59 \cdot 9)\end{array}$ &.$\cdot$ & .. & .. & $\begin{array}{c}21 \cdot 7+5 \\
(0 \cdot 0-44 \cdot 9)\end{array}$ & $\begin{array}{l}65 \cdot 4 \dagger \\
(37 \cdot 6-93 \cdot 3)\end{array}$ \\
\hline 2005-09 &. & $\begin{array}{l}39 \cdot 2+5 \\
(12 \cdot 8-65 \cdot 5)\end{array}$ & $\begin{array}{l}52 \cdot 3+5 \\
(24 \cdot 3-80 \cdot 4)\end{array}$ & $\begin{array}{c}39 \cdot 3+5 \\
(7 \cdot 6-70 \cdot 9)\end{array}$ & .. & $\begin{array}{c}0.6+5 \\
(0.0-1 \cdot 9)\end{array}$ & $\begin{array}{c}19 \cdot 6+\S \\
(8 \cdot 0-31 \cdot 1)\end{array}$ & $\begin{array}{l}100 \cdot 0+5 \\
(88 \cdot 5-100 \cdot 0)\end{array}$ \\
\hline 2010-14 & & .. &. &. & .. & .. & & \\
\hline \multicolumn{9}{|l|}{ Iceland* } \\
\hline $2000-04$ & $\begin{array}{c}13 \cdot 1 \dagger \\
(3 \cdot 8-22 \cdot 4)\end{array}$ & $\begin{array}{l}32 \cdot 9 \\
(24 \cdot 9-40 \cdot 9)\end{array}$ & $\begin{array}{l}61 \cdot 4 \\
(55 \cdot 4-67 \cdot 4)\end{array}$ & $\begin{array}{l}71 \cdot 0 \\
(63 \cdot 0-79 \cdot 0)\end{array}$ & $\begin{array}{c}3.9 \dagger \\
(0.0-10.0)\end{array}$ & $\begin{array}{c}4.8 \\
(1.4-8 \cdot 1)\end{array}$ & $\begin{array}{l}14 \cdot 1 \\
(11 \cdot 2-16 \cdot 9)\end{array}$ & $\begin{array}{l}84 \cdot 5 \\
(77 \cdot 7-91 \cdot 3)\end{array}$ \\
\hline 2005-09 & $\begin{array}{c}19 \cdot 1 \dagger \\
(10 \cdot 4-27 \cdot 8)\end{array}$ & $\begin{array}{l}30 \cdot 3 \\
(21 \cdot 9-38 \cdot 6)\end{array}$ & $\begin{array}{l}64.0 \\
(58.8-69 \cdot 1)\end{array}$ & $\begin{array}{l}66 \cdot 3 \\
(58 \cdot 2-74 \cdot 5)\end{array}$ & $\begin{array}{l}16 \cdot 0 \dagger \\
(6 \cdot 0-26 \cdot 1)\end{array}$ & $\begin{array}{c}0.0 \\
(0.0-0.1)\end{array}$ & $\begin{array}{l}16 \cdot 0 \\
(12 \cdot 9-19 \cdot 1)\end{array}$ & $\begin{array}{l}85 \cdot 4 \\
(79 \cdot 5-91 \cdot 3)\end{array}$ \\
\hline 2010-14 & $\begin{array}{l}17 \cdot 7 \\
(10 \cdot 5-24 \cdot 8)\end{array}$ & $\begin{array}{l}28 \cdot 1 \\
(20 \cdot 2-36 \cdot 1)\end{array}$ & $\begin{array}{l}68 \cdot 2 \\
(63 \cdot 2-73 \cdot 1)\end{array}$ & $\begin{array}{l}63.0 \\
(55 \cdot 5-70.5)\end{array}$ & $\begin{array}{l}14 \cdot 3 \\
(6 \cdot 9-21 \cdot 7)\end{array}$ & $\begin{array}{c}0.0 \dagger \\
(0.0-0.0)\end{array}$ & $\begin{array}{l}20 \cdot 2 \\
(16 \cdot 9-23 \cdot 4)\end{array}$ & $\begin{array}{l}87.5 \\
(82.1-92.9)\end{array}$ \\
\hline \multicolumn{9}{|l|}{ Ireland* } \\
\hline $2000-04$ & $\begin{array}{l}12 \cdot 9 \\
(11 \cdot 0-14 \cdot 7)\end{array}$ & $\begin{array}{l}18 \cdot 6 \\
(16 \cdot 7-20 \cdot 4)\end{array}$ & $\begin{array}{l}53 \cdot 3 \\
(51 \cdot 8-54 \cdot 7)\end{array}$ & $\begin{array}{l}51 \cdot 1 \\
(48 \cdot 9-53 \cdot 2)\end{array}$ & $\begin{array}{c}11 \cdot 6 \\
(8 \cdot 7-14 \cdot 6)\end{array}$ & $\begin{array}{c}5 \cdot 7 \\
(4 \cdot 4-7 \cdot 0)\end{array}$ & $\begin{array}{l}10 \cdot 1 \\
(9 \cdot 3-10 \cdot 9)\end{array}$ & $\begin{array}{l}85 \cdot 5 \\
(83 \cdot 6-87 \cdot 4)\end{array}$ \\
\hline 2005-09 & $\begin{array}{l}17 \cdot 2 \\
(15 \cdot 2-19 \cdot 1)\end{array}$ & $\begin{array}{l}22 \cdot 2 \\
(20 \cdot 4-24 \cdot 1)\end{array}$ & $\begin{array}{l}58 \cdot 4 \\
(57 \cdot 1-59 \cdot 7)\end{array}$ & $\begin{array}{l}57 \cdot 1 \\
(55 \cdot 0-59 \cdot 1)\end{array}$ & $\begin{array}{l}11 \cdot 8 \\
(9 \cdot 4-14 \cdot 2)\end{array}$ & $\begin{array}{c}6 \cdot 9 \\
(5 \cdot 6-8 \cdot 2)\end{array}$ & $\begin{array}{l}13 \cdot 5 \\
(12 \cdot 7-14 \cdot 3)\end{array}$ & $\begin{array}{l}86 \cdot 6 \\
(85 \cdot 1-88 \cdot 1)\end{array}$ \\
\hline 2010-14 & $\begin{array}{l}20 \cdot 3 \\
(18 \cdot 0-22 \cdot 7)\end{array}$ & $\begin{array}{l}27 \cdot 6 \\
(25 \cdot 4-29 \cdot 8)\end{array}$ & $\begin{array}{l}60 \cdot 5 \\
(59 \cdot 1-62 \cdot 0)\end{array}$ & $\begin{array}{l}61 \cdot 7 \\
(59 \cdot 4-64 \cdot 0)\end{array}$ & $\begin{array}{l}14 \cdot 2 \\
(11 \cdot 2-17 \cdot 2)\end{array}$ & $\begin{array}{c}9.6 \\
(7 \cdot 9-11 \cdot 4)\end{array}$ & $\begin{array}{l}17 \cdot 5 \\
(16 \cdot 5-18 \cdot 5)\end{array}$ & $\begin{array}{l}89 \cdot 2 \\
(87 \cdot 7-90 \cdot 7)\end{array}$ \\
\hline \multicolumn{9}{|c|}{ Italy (45 registries) } \\
\hline $2000-04$ & $\begin{array}{l}11 \cdot 5 \\
(10 \cdot 5-12 \cdot 6)\end{array}$ & $\begin{array}{l}31 \cdot 6 \\
(30 \cdot 9-32 \cdot 2)\end{array}$ & $\begin{array}{l}59 \cdot 0 \\
(58 \cdot 6-59 \cdot 5)\end{array}$ & $\begin{array}{l}55 \cdot 8 \\
(54 \cdot 9-56 \cdot 6)\end{array}$ & $\begin{array}{l}15 \cdot 9 \\
(15 \cdot 2-16 \cdot 5)\end{array}$ & $\begin{array}{c}6 \cdot 8 \\
(6 \cdot 3-7 \cdot 3)\end{array}$ & $\begin{array}{l}14 \cdot 0 \\
(13 \cdot 7-14 \cdot 3)\end{array}$ & $\begin{array}{l}84 \cdot 1 \\
(83 \cdot 3-84 \cdot 8)\end{array}$ \\
\hline 2005-09 & $\begin{array}{l}12 \cdot 9 \\
(11 \cdot 9-14 \cdot 0)\end{array}$ & $\begin{array}{c}31 \cdot 1 \\
(30 \cdot 5-31 \cdot 7)\end{array}$ & $\begin{array}{l}64 \cdot 3 \\
(63 \cdot 9-64 \cdot 7)\end{array}$ & $\begin{array}{l}61 \cdot 1 \\
(60 \cdot 4-61 \cdot 8)\end{array}$ & $\begin{array}{l}20 \cdot 0 \\
(19 \cdot 4-20 \cdot 6)\end{array}$ & $\begin{array}{c}7.9 \\
(7 \cdot 5-8 \cdot 3)\end{array}$ & $\begin{array}{l}15 \cdot 5 \\
(15 \cdot 2-15 \cdot 8)\end{array}$ & $\begin{array}{l}86 \cdot 0 \\
(85 \cdot 5-86 \cdot 6)\end{array}$ \\
\hline 2010-14 & $\begin{array}{l}13 \cdot 8 \\
(12 \cdot 3-15 \cdot 3)\end{array}$ & $\begin{array}{l}30 \cdot 5 \\
(29 \cdot 7-31 \cdot 3)\end{array}$ & $\begin{array}{l}64 \cdot 2 \\
(63 \cdot 6-64 \cdot 7)\end{array}$ & $\begin{array}{l}61 \cdot 3 \\
(60 \cdot 3-62 \cdot 2)\end{array}$ & $\begin{array}{l}20 \cdot 3 \\
(19 \cdot 6-21 \cdot 1)\end{array}$ & $\begin{array}{c}9.2 \\
(8 \cdot 5-9.8)\end{array}$ & $\begin{array}{l}15 \cdot 9 \\
(15 \cdot 5-16 \cdot 3)\end{array}$ & $\begin{array}{l}85 \cdot 7 \\
(85 \cdot 0-86 \cdot 5)\end{array}$ \\
\hline \multicolumn{9}{|l|}{ Latvia* } \\
\hline $2000-04$ & $\begin{array}{c}6.9 \\
(3 \cdot 4-10 \cdot 3)\end{array}$ & $\begin{array}{l}24 \cdot 2 \\
(22 \cdot 2-26 \cdot 3)\end{array}$ & $\begin{array}{l}50 \cdot 5 \\
(47 \cdot 8-53 \cdot 3)\end{array}$ & $\begin{array}{l}39 \cdot 4 \\
(36 \cdot 3-42 \cdot 5)\end{array}$ & $\begin{array}{c}12 \cdot 0 \dagger \\
(3 \cdot 8-20 \cdot 3)\end{array}$ & $\begin{array}{c}8.8 \\
(5 \cdot 4-12 \cdot 1)\end{array}$ & $\begin{array}{c}17 \cdot 3 \\
(15 \cdot 3-19 \cdot 3)\end{array}$ & $\begin{array}{l}66 \cdot 3 \\
(62 \cdot 0-70 \cdot 6)\end{array}$ \\
\hline 2005-09 & $\begin{array}{l}10 \cdot 8 \\
(7 \cdot 3-14 \cdot 2)\end{array}$ & $\begin{array}{l}26 \cdot 7 \\
(24 \cdot 6-28 \cdot 9)\end{array}$ & $\begin{array}{l}54 \cdot 0 \\
(51 \cdot 4-56 \cdot 5)\end{array}$ & $\begin{array}{l}46 \cdot 6 \\
(43 \cdot 4-49 \cdot 7)\end{array}$ & $\begin{array}{c}7 \cdot 8 \\
(3 \cdot 8-11 \cdot 7)\end{array}$ & $\begin{array}{c}11 \cdot 2 \\
(7 \cdot 5-14 \cdot 8)\end{array}$ & $\begin{array}{l}19 \cdot 5 \\
(17 \cdot 7-21 \cdot 2)\end{array}$ & $\begin{array}{l}65 \cdot 1 \\
(61 \cdot 1-69 \cdot 1)\end{array}$ \\
\hline 2010-14 & $\begin{array}{c}6 \cdot 1 \\
(3 \cdot 6-8 \cdot 6)\end{array}$ & $\begin{array}{l}28 \cdot 0 \\
(25 \cdot 7-30 \cdot 3)\end{array}$ & $\begin{array}{l}56.5 \\
(54.0-58.9)\end{array}$ & $\begin{array}{l}53 \cdot 3 \\
(50 \cdot 2-56 \cdot 5)\end{array}$ & $\begin{array}{l}12 \cdot 9 \\
(7 \cdot 7-18.0)\end{array}$ & $\begin{array}{l}13 \cdot 7 \\
(9 \cdot 3-18 \cdot 1)\end{array}$ & $\begin{array}{l}20 \cdot 4 \\
(18 \cdot 7-22 \cdot 1)\end{array}$ & $\begin{array}{l}72 \cdot 1 \\
(68 \cdot 3-75 \cdot 8)\end{array}$ \\
\hline \multicolumn{9}{|l|}{ Lithuania* } \\
\hline 2000-04 & $\begin{array}{c}4 \cdot 7 \\
(2 \cdot 9-6 \cdot 4)\end{array}$ & $\begin{array}{l}22 \cdot 0 \\
(20 \cdot 7-23 \cdot 3)\end{array}$ & $\begin{array}{l}44 \cdot 5 \\
(42 \cdot 6-46 \cdot 3)\end{array}$ & $\begin{array}{l}40 \cdot 6 \\
(38 \cdot 4-42 \cdot 8)\end{array}$ & $\begin{array}{c}7 \cdot 9 \\
(5 \cdot 6-10 \cdot 2)\end{array}$ & $\begin{array}{c}6 \cdot 5 \\
(5 \cdot 3-7 \cdot 7)\end{array}$ & $\begin{array}{c}8 \cdot 8 \\
(8 \cdot 0-9 \cdot 6)\end{array}$ & $\begin{array}{l}67 \cdot 2 \\
(63 \cdot 9-70 \cdot 6)\end{array}$ \\
\hline 2005-09 & $\begin{array}{c}6 \cdot 2 \\
(4 \cdot 2-8 \cdot 1)\end{array}$ & $\begin{array}{l}24 \cdot 9 \\
(23 \cdot 4-26 \cdot 4)\end{array}$ & $\begin{array}{l}51 \cdot 1 \\
(49 \cdot 2-53 \cdot 0)\end{array}$ & $\begin{array}{l}48 \cdot 8 \\
(46 \cdot 6-51 \cdot 1)\end{array}$ & $\begin{array}{c}8.35 \\
(5 \cdot 9-10 \cdot 7)\end{array}$ & $\begin{array}{c}5 \cdot 4 \S \\
(4 \cdot 3-6 \cdot 5)\end{array}$ & $\begin{array}{c}8 \cdot 8 \\
(8 \cdot 0-9 \cdot 7)\end{array}$ & $\begin{array}{l}70 \cdot 6 \\
(67 \cdot 6-73 \cdot 7)\end{array}$ \\
\hline 2010-14 & $\begin{array}{c}5 \cdot 6 \\
(3 \cdot 3-7 \cdot 8)\end{array}$ & $\begin{array}{l}27 \cdot 0 \\
(24 \cdot 9-29 \cdot 0)\end{array}$ & $\begin{array}{l}56 \cdot 9 \\
(54 \cdot 4-59 \cdot 4)\end{array}$ & $\begin{array}{l}52 \cdot 7 \\
(49 \cdot 7-55 \cdot 7)\end{array}$ & $\begin{array}{c}8.05 \\
(5 \cdot 3-10 \cdot 7)\end{array}$ & $\begin{array}{c}7.05 \\
(5 \cdot 2-8 \cdot 8)\end{array}$ & $\begin{array}{c}9 \cdot 9 \\
(8 \cdot 7-11 \cdot 0)\end{array}$ & $\begin{array}{l}75 \cdot 3 \\
(71 \cdot 8-78 \cdot 9)\end{array}$ \\
\hline \multicolumn{9}{|l|}{ Malta* } \\
\hline $2000-04$ & $\begin{array}{c}7.8 \dagger \\
(0.0-15 \cdot 6)\end{array}$ & $\begin{array}{l}19 \cdot 9 \\
(14 \cdot 6-25 \cdot 2)\end{array}$ & $\begin{array}{l}57 \cdot 0 \\
(51 \cdot 9-62 \cdot 2)\end{array}$ & $\begin{array}{l}55 \cdot 1 \\
(47 \cdot 7-62 \cdot 5)\end{array}$ & $\begin{array}{c}4 \cdot 2 \dagger \\
(0 \cdot 0-10 \cdot 5)\end{array}$ & $\begin{array}{c}5.9 \\
(3 \cdot 0-8 \cdot 7)\end{array}$ & $\begin{array}{c}9 \cdot 2 \\
(6 \cdot 5-12 \cdot 0)\end{array}$ & $\begin{array}{l}87 \cdot 3 \\
(80 \cdot 0-94 \cdot 7)\end{array}$ \\
\hline 2005-09 & $\begin{array}{c}6.1 \dagger \\
(0.5-11 \cdot 8)\end{array}$ & $\begin{array}{l}24 \cdot 8 \\
(19 \cdot 3-30 \cdot 3)\end{array}$ & $\begin{array}{l}52 \cdot 9 \\
(48 \cdot 5-57 \cdot 4)\end{array}$ & $\begin{array}{l}53 \cdot 8 \\
(47 \cdot 2-60 \cdot 5)\end{array}$ & $\begin{array}{c}0.0+5 \\
(0.0-0.0)\end{array}$ & $\begin{array}{c}2.25 \\
(0.5-4 \cdot 0)\end{array}$ & $\begin{array}{l}11 \cdot 4 \\
(8 \cdot 7-14 \cdot 1)\end{array}$ & $\begin{array}{l}81 \cdot 6 \\
(75 \cdot 2-88.1)\end{array}$ \\
\hline \multirow[t]{2}{*}{ 2010-14 } & $\begin{array}{l}11 \cdot 2 \dagger \\
(1 \cdot 6-20 \cdot 8)\end{array}$ & $\begin{array}{l}23 \cdot 8 \\
(17 \cdot 5-30 \cdot 0)\end{array}$ & $\begin{array}{l}57 \cdot 5 \\
(52 \cdot 6-62 \cdot 3)\end{array}$ & $\begin{array}{l}56 \cdot 1 \\
(49 \cdot 2-62 \cdot 9)\end{array}$ & $\begin{array}{c}0.0+5 \\
(0.0-0.0)\end{array}$ & $\begin{array}{c}5 \cdot 5 \S \\
(2 \cdot 7-8 \cdot 3)\end{array}$ & $\begin{array}{l}14 \cdot 9 \\
(11 \cdot 5-18 \cdot 2)\end{array}$ & $\begin{array}{l}81 \cdot 9 \\
(75 \cdot 6-88 \cdot 3)\end{array}$ \\
\hline & & & & & & & \multicolumn{2}{|c|}{ (Table 6 continues on next page } \\
\hline
\end{tabular}




\begin{tabular}{|c|c|c|c|c|c|c|c|c|}
\hline & \multicolumn{6}{|c|}{ Gastrointestinal cancers } & \multirow[t]{2}{*}{ Lung } & \multirow{2}{*}{$\begin{array}{l}\text { Melanoma of } \\
\text { the skin }\end{array}$} \\
\hline & Oesophagus & Stomach & Colon & Rectum & Liver & Pancreas & & \\
\hline \multicolumn{9}{|c|}{ (Continued from previous page) } \\
\hline \multicolumn{9}{|c|}{ Netherlands* } \\
\hline $2000-04$ & $\begin{array}{l}12 \cdot 1 \\
(11 \cdot 2-13 \cdot 0)\end{array}$ & $\begin{array}{l}19 \cdot 7 \\
(18 \cdot 8-20 \cdot 6)\end{array}$ & $\begin{array}{l}58 \cdot 1 \\
(57 \cdot 4-58 \cdot 7)\end{array}$ & $\begin{array}{l}58 \cdot 0 \\
(57 \cdot 0-59 \cdot 1)\end{array}$ & $\begin{array}{l}10 \cdot 1 \\
(8 \cdot 6-11 \cdot 7)\end{array}$ & $\begin{array}{c}3 \cdot 7 \\
(3 \cdot 2-4 \cdot 2)\end{array}$ & $\begin{array}{l}12 \cdot 4 \\
(12 \cdot 1-12 \cdot 7)\end{array}$ & $\begin{array}{l}88 \cdot 4 \\
(87 \cdot 6-89 \cdot 2)\end{array}$ \\
\hline 2005-09 & $\begin{array}{l}16 \cdot 8 \\
(15 \cdot 8-17 \cdot 7)\end{array}$ & $\begin{array}{l}22 \cdot 9 \\
(22 \cdot 0-23 \cdot 9)\end{array}$ & $\begin{array}{l}60 \cdot 9 \\
(60 \cdot 3-61 \cdot 5)\end{array}$ & $\begin{array}{l}63 \cdot 1 \\
(62 \cdot 2-64 \cdot 0)\end{array}$ & $\begin{array}{l}13 \cdot 4 \\
(11 \cdot 8-14 \cdot 9)\end{array}$ & $\begin{array}{c}5 \cdot 5 \\
(5 \cdot 0-6 \cdot 1)\end{array}$ & $\begin{array}{l}15 \cdot 7 \\
(15 \cdot 3-16 \cdot 0)\end{array}$ & $\begin{array}{l}89 \cdot 8 \\
(89 \cdot 1-90 \cdot 4)\end{array}$ \\
\hline 2010-14 & $\begin{array}{l}21 \cdot 0 \\
(20 \cdot 0-22 \cdot 0)\end{array}$ & $\begin{array}{l}25 \cdot 0 \\
(24 \cdot 0-26 \cdot 0)\end{array}$ & $\begin{array}{l}63 \cdot 1 \\
(62 \cdot 5-63 \cdot 7)\end{array}$ & $\begin{array}{l}65 \cdot 3 \\
(64 \cdot 5-66 \cdot 2)\end{array}$ & $\begin{array}{l}15 \cdot 8 \\
(14 \cdot 2-17 \cdot 4)\end{array}$ & $\begin{array}{c}7 \cdot 4 \\
(6 \cdot 8-8 \cdot 1)\end{array}$ & $\begin{array}{l}17 \cdot 3 \\
(16 \cdot 9-17 \cdot 6)\end{array}$ & $\begin{array}{l}91 \cdot 0 \\
(90 \cdot 4-91 \cdot 6)\end{array}$ \\
\hline \multicolumn{9}{|l|}{ Norway* } \\
\hline 2000-04 & $\begin{array}{c}9.0 \\
(6 \cdot 7-11 \cdot 3)\end{array}$ & $\begin{array}{l}22 \cdot 4 \\
(20 \cdot 5-24 \cdot 2)\end{array}$ & $\begin{array}{l}60 \cdot 0 \\
(58 \cdot 8-61 \cdot 2)\end{array}$ & $\begin{array}{l}62 \cdot 4 \\
(60 \cdot 8-64 \cdot 1)\end{array}$ & $\begin{array}{c}7 \cdot 9 \\
(5 \cdot 4-10 \cdot 3)\end{array}$ & $\begin{array}{c}5 \cdot 0 \\
(3 \cdot 9-6 \cdot 0)\end{array}$ & $\begin{array}{l}12 \cdot 3 \\
(11 \cdot 5-13 \cdot 1)\end{array}$ & $\begin{array}{l}86 \cdot 4 \\
(85 \cdot 1-87 \cdot 6)\end{array}$ \\
\hline 2005-09 & $\begin{array}{l}13 \cdot 3 \\
(10 \cdot 8-15 \cdot 8)\end{array}$ & $\begin{array}{l}25 \cdot 3 \\
(23 \cdot 3-27 \cdot 4)\end{array}$ & $\begin{array}{l}64 \cdot 3 \\
(63 \cdot 2-65 \cdot 4)\end{array}$ & $\begin{array}{l}66 \cdot 8 \\
(65 \cdot 1-68 \cdot 4)\end{array}$ & $\begin{array}{l}14 \cdot 2 \\
(11 \cdot 2-17 \cdot 2)\end{array}$ & $\begin{array}{c}6 \cdot 1 \\
(5 \cdot 0-7 \cdot 2)\end{array}$ & $\begin{array}{l}15 \cdot 9 \\
(15 \cdot 1-16 \cdot 8)\end{array}$ & $\begin{array}{l}87 \cdot 3 \\
(86 \cdot 2-88 \cdot 3)\end{array}$ \\
\hline $2010-14$ & $\begin{array}{l}16 \cdot 5 \\
(14 \cdot 0-19 \cdot 0)\end{array}$ & $\begin{array}{l}26 \cdot 5 \\
(24 \cdot 4-28 \cdot 6)\end{array}$ & $\begin{array}{l}66 \cdot 7 \\
(65 \cdot 6-67 \cdot 8)\end{array}$ & $\begin{array}{l}69 \cdot 2 \\
(67 \cdot 6-70 \cdot 8)\end{array}$ & $\begin{array}{l}18 \cdot 7 \\
(15 \cdot 5-22 \cdot 0)\end{array}$ & $\begin{array}{c}9.5 \\
(8 \cdot 0-11 \cdot 0)\end{array}$ & $\begin{array}{l}19 \cdot 0 \\
(18 \cdot 1-19 \cdot 8)\end{array}$ & $\begin{array}{l}89 \cdot 3 \\
(88 \cdot 3-90 \cdot 3)\end{array}$ \\
\hline \multicolumn{9}{|c|}{ Poland (16 registries)* } \\
\hline $2000-04$ & $\begin{array}{c}7 \cdot 2 \\
(6 \cdot 3-8 \cdot 1)\end{array}$ & $\begin{array}{l}15 \cdot 9 \\
(15 \cdot 2-16 \cdot 5)\end{array}$ & $\begin{array}{l}45 \cdot 3 \\
(44 \cdot 6-45 \cdot 9)\end{array}$ & $\begin{array}{l}42 \cdot 5 \\
(41 \cdot 8-43 \cdot 3)\end{array}$ & $\begin{array}{c}9.5 \\
(8 \cdot 5-10 \cdot 5)\end{array}$ & $\begin{array}{c}8 \cdot 2 \\
(7 \cdot 6-8 \cdot 8)\end{array}$ & $\begin{array}{l}12 \cdot 1 \\
(11 \cdot 8-12 \cdot 4)\end{array}$ & $\begin{array}{l}63 \cdot 2 \\
(62 \cdot 0-64 \cdot 4)\end{array}$ \\
\hline 2005-09 & $\begin{array}{c}8 \cdot 9 \\
(7 \cdot 9-9 \cdot 8)\end{array}$ & $\begin{array}{l}19 \cdot 9 \\
(19 \cdot 3-20 \cdot 4)\end{array}$ & $\begin{array}{l}51 \cdot 1 \\
(50 \cdot 5-51 \cdot 6)\end{array}$ & $\begin{array}{l}47 \cdot 6 \\
(46 \cdot 9-48 \cdot 3)\end{array}$ & $\begin{array}{l}10 \cdot 7 \\
(9 \cdot 8-11 \cdot 7)\end{array}$ & $\begin{array}{c}9 \cdot 7 \\
(9 \cdot 2-10 \cdot 3)\end{array}$ & $\begin{array}{l}14 \cdot 1 \\
(13 \cdot 8-14 \cdot 4)\end{array}$ & $\begin{array}{l}67 \cdot 1 \\
(66 \cdot 2-68 \cdot 1)\end{array}$ \\
\hline 2010-14 & $\begin{array}{c}9 \cdot 1 \\
(8 \cdot 1-10 \cdot 1)\end{array}$ & $\begin{array}{l}20 \cdot 9 \\
(20 \cdot 3-21 \cdot 4)\end{array}$ & $\begin{array}{l}52 \cdot 9 \\
(52 \cdot 3-53 \cdot 4)\end{array}$ & $\begin{array}{l}48 \cdot 4 \\
(47 \cdot 7-49 \cdot 1)\end{array}$ & $\begin{array}{l}10 \cdot 8 \\
(9 \cdot 9-11 \cdot 8)\end{array}$ & $\begin{array}{c}8 \cdot 0 \\
(7 \cdot 5-8 \cdot 5)\end{array}$ & $\begin{array}{l}14 \cdot 4 \\
(14 \cdot 1-14 \cdot 7)\end{array}$ & $\begin{array}{l}69 \cdot 8 \\
(68 \cdot 9-70 \cdot 7)\end{array}$ \\
\hline \multicolumn{9}{|c|}{ Portugal (four registries)* } \\
\hline 2000-04 & $\begin{array}{l}10 \cdot 2 \\
(8 \cdot 6-11 \cdot 7)\end{array}$ & $\begin{array}{l}29.8 \\
(28 \cdot 9-30 \cdot 8)\end{array}$ & $\begin{array}{l}56 \cdot 5 \\
(55 \cdot 6-57 \cdot 4)\end{array}$ & $\begin{array}{l}54 \cdot 4 \\
(53 \cdot 1-55 \cdot 7)\end{array}$ & $\begin{array}{l}13 \cdot 6 \\
(11 \cdot 8-15 \cdot 5)\end{array}$ & $\begin{array}{c}8 \cdot 0 \\
(6 \cdot 7-9 \cdot 2)\end{array}$ & $\begin{array}{l}10 \cdot 6 \\
(10 \cdot 0-11 \cdot 2)\end{array}$ & $\begin{array}{l}78 \cdot 8 \\
(76 \cdot 8-80 \cdot 7)\end{array}$ \\
\hline 2005-09 & $\begin{array}{l}12 \cdot 5 \\
(11 \cdot 0-14 \cdot 1)\end{array}$ & $\begin{array}{l}32 \cdot 4 \\
(31 \cdot 5-33 \cdot 3)\end{array}$ & $\begin{array}{l}61 \cdot 1 \\
(60 \cdot 4-61 \cdot 9)\end{array}$ & $\begin{array}{l}59 \cdot 8 \\
(58 \cdot 7-60 \cdot 9)\end{array}$ & $\begin{array}{l}15 \cdot 3 \\
(13 \cdot 6-17 \cdot 0)\end{array}$ & $\begin{array}{l}10 \cdot 3 \\
(9 \cdot 1-11 \cdot 4)\end{array}$ & $\begin{array}{l}14 \cdot 1 \\
(13 \cdot 5-14 \cdot 7)\end{array}$ & $\begin{array}{l}81 \cdot 7 \\
(80 \cdot 2-83 \cdot 2)\end{array}$ \\
\hline 2010-14 & $\begin{array}{l}16 \cdot 1 \\
(12 \cdot 0-20 \cdot 2)\end{array}$ & $\begin{array}{l}32 \cdot 2 \\
(30 \cdot 2-34 \cdot 2)\end{array}$ & $\begin{array}{l}60 \cdot 9 \\
(59 \cdot 2-62 \cdot 6)\end{array}$ & $\begin{array}{l}59 \cdot 6 \\
(57 \cdot 1-62 \cdot 1)\end{array}$ & $\begin{array}{l}18 \cdot 7 \\
(14 \cdot 5-22 \cdot 9)\end{array}$ & $\begin{array}{l}10 \cdot 7 \\
(8 \cdot 2-13 \cdot 1)\end{array}$ & $\begin{array}{l}15 \cdot 7 \\
(14 \cdot 3-17 \cdot 1)\end{array}$ & $\begin{array}{l}83 \cdot 7 \\
(80 \cdot 4-87 \cdot 0)\end{array}$ \\
\hline \multicolumn{9}{|c|}{ Romania (Cluj) } \\
\hline 2000-04 &.$\cdot$ &.. &.$\cdot$ &.. &.$\cdot$ &.. &.. &.. \\
\hline 2005-09 & $\begin{array}{l}10 \cdot 25 \\
(4 \cdot 4-16 \cdot 0)\end{array}$ & $\begin{array}{l}19 \cdot 35 \\
(16 \cdot 0-22 \cdot 7)\end{array}$ & $\begin{array}{l}53.5 \$ \\
(48 \cdot 9-58 \cdot 0)\end{array}$ & $\begin{array}{l}44 \cdot 8 \\
(38 \cdot 9-50 \cdot 8)\end{array}$ & $\begin{array}{c}4 \cdot 15 \\
(1 \cdot 8-6 \cdot 4)\end{array}$ & $\begin{array}{c}4 \cdot 55 \\
(2 \cdot 2-6 \cdot 7)\end{array}$ & $\begin{array}{c}7 \cdot 95 \\
(6 \cdot 1-9 \cdot 6)\end{array}$ & $\begin{array}{l}68 \cdot 4 \\
(61 \cdot 5-75 \cdot 3)\end{array}$ \\
\hline 2010-14 & $\begin{array}{c}0.0+5 \\
(0.0-0.1)\end{array}$ & $\begin{array}{l}26 \cdot 05 \\
(21 \cdot 5-30 \cdot 5)\end{array}$ & $\begin{array}{l}52 \cdot 25 \\
(46 \cdot 0-58 \cdot 4)\end{array}$ & $\begin{array}{l}58 \cdot 4 \\
(49 \cdot 8-67 \cdot 0)\end{array}$ & $\begin{array}{l}13 \cdot 25 \\
(9 \cdot 2-17 \cdot 3)\end{array}$ & $\begin{array}{c}6 \cdot 0+5 \\
(2 \cdot 6-9 \cdot 3)\end{array}$ & $\begin{array}{l}11 \cdot 15 \\
(8 \cdot 7-13 \cdot 6)\end{array}$ & $\begin{array}{l}71 \cdot 3 \\
(63 \cdot 0-79 \cdot 6)\end{array}$ \\
\hline \multicolumn{9}{|c|}{ Russia (five registries) } \\
\hline $2000-04$ & $\begin{array}{l}10 \cdot 9 \\
(8 \cdot 8-13 \cdot 0)\end{array}$ & $\begin{array}{l}22 \cdot 9 \\
(21 \cdot 7-24 \cdot 1)\end{array}$ & $\begin{array}{l}40 \cdot 4 \\
(38 \cdot 9-41 \cdot 9)\end{array}$ & $\begin{array}{l}38 \cdot 5 \\
(36 \cdot 4-40 \cdot 6)\end{array}$ & $\begin{array}{c}7 \cdot 4 \\
(5 \cdot 2-9 \cdot 5)\end{array}$ & $\begin{array}{c}7 \cdot 5 \\
(6 \cdot 0-9 \cdot 1)\end{array}$ & $\begin{array}{l}16 \cdot 8 \\
(15 \cdot 7-17 \cdot 8)\end{array}$ & $\begin{array}{l}64 \cdot 7 \\
(61 \cdot 4-68 \cdot 0)\end{array}$ \\
\hline $2005-09$ & $\begin{array}{c}8 \cdot 6 \\
(7 \cdot 0-10 \cdot 2)\end{array}$ & $\begin{array}{l}20 \cdot 2 \\
(19 \cdot 3-21 \cdot 1)\end{array}$ & $\begin{array}{l}42 \cdot 4 \\
(41 \cdot 2-43 \cdot 6)\end{array}$ & $\begin{array}{l}38 \cdot 9 \\
(37 \cdot 3-40 \cdot 5)\end{array}$ & $\begin{array}{c}5 \cdot 1 \\
(3 \cdot 6-6 \cdot 6)\end{array}$ & $\begin{array}{c}5 \cdot 4 \\
(4 \cdot 5-6 \cdot 4)\end{array}$ & $\begin{array}{l}13 \cdot 9 \\
(13 \cdot 2-14 \cdot 6)\end{array}$ & $\begin{array}{l}63 \cdot 3 \\
(60 \cdot 3-66 \cdot 3)\end{array}$ \\
\hline 2010-14 & $\begin{array}{c}8 \cdot 6 \\
(7 \cdot 1-10 \cdot 1)\end{array}$ & $\begin{array}{l}21 \cdot 0 \\
(20 \cdot 0-21 \cdot 9)\end{array}$ & $\begin{array}{l}44 \cdot 9 \\
(43 \cdot 8-46 \cdot 1)\end{array}$ & $\begin{array}{l}41 \cdot 9 \\
(40 \cdot 2-43 \cdot 5)\end{array}$ & $\begin{array}{c}6 \cdot 3 \\
(4 \cdot 8-7 \cdot 9)\end{array}$ & $\begin{array}{c}4 \cdot 4 \\
(3 \cdot 7-5 \cdot 2)\end{array}$ & $\begin{array}{l}13 \cdot 7 \\
(13 \cdot 0-14 \cdot 4)\end{array}$ & $\begin{array}{l}66 \cdot 5 \\
(63 \cdot 6-69 \cdot 3)\end{array}$ \\
\hline \multicolumn{9}{|l|}{ Slovakia* } \\
\hline $2000-04$ & $\begin{array}{c}5 \cdot 85 \\
(3 \cdot 8-7 \cdot 9)\end{array}$ & $\begin{array}{l}20 \cdot 6 \$ \\
(19 \cdot 1-22 \cdot 0)\end{array}$ & $\begin{array}{l}50 \cdot 4 \\
(49 \cdot 1-51 \cdot 7)\end{array}$ & $\begin{array}{l}43 \cdot 6 \\
(41 \cdot 7-45 \cdot 5)\end{array}$ & $\begin{array}{c}5 \cdot 65 \\
(4 \cdot 1-7 \cdot 2)\end{array}$ & $\begin{array}{c}4 \cdot 85 \\
(3 \cdot 8-5 \cdot 8)\end{array}$ & $\begin{array}{c}9 \cdot 55 \\
(8 \cdot 7-10 \cdot 3)\end{array}$ & $\begin{array}{l}75 \cdot 0 \\
(72 \cdot 7-77 \cdot 3)\end{array}$ \\
\hline 2005-09 & $\begin{array}{c}6 \cdot 4 \\
(4 \cdot 7-8 \cdot 0)\end{array}$ & $\begin{array}{l}20 \cdot 9 \\
(19 \cdot 5-22 \cdot 4)\end{array}$ & $\begin{array}{l}51 \cdot 2 \\
(50 \cdot 1-52 \cdot 4)\end{array}$ & $\begin{array}{l}47 \cdot 5 \\
(45 \cdot 8-49 \cdot 2)\end{array}$ & $\begin{array}{c}6 \cdot 15 \\
(4 \cdot 8-7 \cdot 5)\end{array}$ & $\begin{array}{c}5 \cdot 8 \\
(4 \cdot 8-6 \cdot 7)\end{array}$ & $\begin{array}{l}10 \cdot 5 \\
(9 \cdot 7-11 \cdot 2)\end{array}$ & $\begin{array}{l}79 \cdot 7 \\
(77 \cdot 7-81 \cdot 7)\end{array}$ \\
\hline 2010-14 & $\begin{array}{c}6 \cdot 4 \\
(3 \cdot 3-9 \cdot 4)\end{array}$ & $\begin{array}{l}21 \cdot 1 \\
(17 \cdot 8-24 \cdot 3)\end{array}$ & $\begin{array}{l}51 \cdot 8 \\
(49 \cdot 1-54 \cdot 4)\end{array}$ & $\begin{array}{l}48 \cdot 6 \\
(44 \cdot 9-52 \cdot 4)\end{array}$ & $\begin{array}{c}7.65 \\
(4 \cdot 5-10 \cdot 7)\end{array}$ & $\begin{array}{c}6 \cdot 4 \\
(4 \cdot 4-8 \cdot 4)\end{array}$ & $\begin{array}{l}11 \cdot 2 \\
(9 \cdot 5-12 \cdot 8)\end{array}$ & $\begin{array}{l}78 \cdot 2 \\
(73 \cdot 9-82 \cdot 5)\end{array}$ \\
\hline \multicolumn{9}{|l|}{ Slovenia* } \\
\hline 2000-04 & $\begin{array}{c}8 \cdot 2 \\
(5 \cdot 7-10 \cdot 8)\end{array}$ & $\begin{array}{l}25 \cdot 9 \\
(23 \cdot 8-27 \cdot 9)\end{array}$ & $\begin{array}{l}53 \cdot 6 \\
(51 \cdot 6-55 \cdot 6)\end{array}$ & $\begin{array}{l}48 \cdot 6 \\
(46 \cdot 1-51 \cdot 1)\end{array}$ & $\begin{array}{c}3 \cdot 8 \\
(2 \cdot 2-5 \cdot 4)\end{array}$ & $\begin{array}{c}4 \cdot 7 \\
(3 \cdot 4-6 \cdot 1)\end{array}$ & $\begin{array}{c}9 \cdot 9 \\
(9 \cdot 1-10 \cdot 8)\end{array}$ & $\begin{array}{l}79 \cdot 3 \\
(76 \cdot 7-81 \cdot 9)\end{array}$ \\
\hline $2005-09$ & $\begin{array}{c}9 \cdot 3 \\
(6 \cdot 2-12 \cdot 3)\end{array}$ & $\begin{array}{l}25 \cdot 8 \\
(23 \cdot 7-27 \cdot 8)\end{array}$ & $\begin{array}{l}56 \cdot 2 \\
(54 \cdot 4-58 \cdot 0)\end{array}$ & $\begin{array}{l}57 \cdot 0 \\
(54 \cdot 6-59 \cdot 4)\end{array}$ & $\begin{array}{c}7 \cdot 0 \\
(5 \cdot 2-8 \cdot 8)\end{array}$ & $\begin{array}{c}5 \cdot 7 \\
(4 \cdot 3-7 \cdot 1)\end{array}$ & $\begin{array}{l}12 \cdot 7 \\
(11 \cdot 7-13 \cdot 7)\end{array}$ & $\begin{array}{l}85 \cdot 0 \\
(83 \cdot 0-87 \cdot 0)\end{array}$ \\
\hline \multirow[t]{2}{*}{ 2010-14 } & $\begin{array}{c}8 \cdot 6 \\
(5 \cdot 2-11 \cdot 9)\end{array}$ & $\begin{array}{l}28 \cdot 8 \\
(26 \cdot 4-31 \cdot 2)\end{array}$ & $\begin{array}{l}61 \cdot 9 \\
(60 \cdot 0-63 \cdot 8)\end{array}$ & $\begin{array}{l}60 \cdot 3 \\
(57 \cdot 7-62 \cdot 9)\end{array}$ & $\begin{array}{c}7 \cdot 4 \\
(5 \cdot 3-9 \cdot 5)\end{array}$ & $\begin{array}{c}6.6 \\
(4 \cdot 9-8 \cdot 4)\end{array}$ & $\begin{array}{l}14 \cdot 8 \\
(13 \cdot 6-16 \cdot 1)\end{array}$ & $\begin{array}{l}85 \cdot 1 \\
(83 \cdot 0-87 \cdot 1)\end{array}$ \\
\hline & & & & & & & \multicolumn{2}{|c|}{ (Table 6 continues on next pag } \\
\hline
\end{tabular}




\begin{tabular}{|c|c|c|c|c|c|c|c|c|}
\hline & \multicolumn{6}{|c|}{ Gastrointestinal cancers } & \multirow[t]{2}{*}{ Lung } & \multirow{2}{*}{$\begin{array}{l}\text { Melanoma of } \\
\text { the skin }\end{array}$} \\
\hline & Oesophagus & Stomach & Colon & Rectum & Liver & Pancreas & & \\
\hline \multicolumn{9}{|c|}{ (Continued from previous page) } \\
\hline \multicolumn{9}{|c|}{ Spain (ten registries) } \\
\hline 2000-04 & $\begin{array}{c}8 \cdot 9 \\
(7 \cdot 5-10 \cdot 3)\end{array}$ & $\begin{array}{l}25 \cdot 7 \\
(24.5-26 \cdot 9)\end{array}$ & $\begin{array}{l}56 \cdot 5 \\
(55 \cdot 6-57 \cdot 4)\end{array}$ & $\begin{array}{l}55 \cdot 2 \\
(53 \cdot 7-56 \cdot 6)\end{array}$ & $\begin{array}{l}14 \cdot 4 \\
(13 \cdot 2-15 \cdot 7)\end{array}$ & $\begin{array}{c}5 \cdot 6 \\
(4 \cdot 8-6 \cdot 4)\end{array}$ & $\begin{array}{l}10 \cdot 8 \\
(10 \cdot 4-11 \cdot 3)\end{array}$ & $\begin{array}{l}85 \cdot 3 \\
(83 \cdot 8-86 \cdot 8)\end{array}$ \\
\hline 2005-09 & $\begin{array}{l}11 \cdot 6 \\
(10 \cdot 2-13 \cdot 1)\end{array}$ & $\begin{array}{l}26 \cdot 7 \\
(25 \cdot 5-27 \cdot 9)\end{array}$ & $\begin{array}{l}61 \cdot 1 \\
(60 \cdot 3-61 \cdot 9)\end{array}$ & $\begin{array}{l}58 \cdot 6 \\
(57 \cdot 3-59 \cdot 9)\end{array}$ & $\begin{array}{l}16 \cdot 3 \\
(15 \cdot 1-17 \cdot 4)\end{array}$ & $\begin{array}{c}6 \cdot 9 \\
(6 \cdot 1-7 \cdot 7)\end{array}$ & $\begin{array}{l}12 \cdot 5 \\
(12 \cdot 0-13 \cdot 0)\end{array}$ & $\begin{array}{l}87 \cdot 1 \\
(85 \cdot 9-88 \cdot 3)\end{array}$ \\
\hline 2010-14 & $\begin{array}{l}13 \cdot 0 \\
(10 \cdot 6-15 \cdot 4)\end{array}$ & $\begin{array}{l}27 \cdot 6 \\
(25 \cdot 7-29 \cdot 5)\end{array}$ & $\begin{array}{l}63 \cdot 2 \\
(62 \cdot 0-64 \cdot 5)\end{array}$ & $\begin{array}{l}59 \cdot 5 \\
(57 \cdot 4-61 \cdot 5)\end{array}$ & $\begin{array}{l}17 \cdot 3 \\
(15 \cdot 4-19 \cdot 2)\end{array}$ & $\begin{array}{c}7 \cdot 7 \\
(6 \cdot 3-9 \cdot 2)\end{array}$ & $\begin{array}{l}13 \cdot 5 \\
(12 \cdot 7-14 \cdot 3)\end{array}$ & $\begin{array}{l}86 \cdot 8 \\
(84 \cdot 8-88 \cdot 7)\end{array}$ \\
\hline \multicolumn{9}{|l|}{ Sweden* } \\
\hline $2000-04$ & $\begin{array}{l}11 \cdot 4 \\
(9 \cdot 6-13 \cdot 1)\end{array}$ & $\begin{array}{l}21 \cdot 2 \\
(19 \cdot 8-22 \cdot 6)\end{array}$ & $\begin{array}{l}60 \cdot 2 \\
(59 \cdot 2-61 \cdot 2)\end{array}$ & $\begin{array}{l}59 \cdot 9 \\
(58 \cdot 7-61 \cdot 1)\end{array}$ & $\begin{array}{c}7 \cdot 8 \\
(6 \cdot 4-9 \cdot 1)\end{array}$ & $\begin{array}{c}4 \cdot 9 \\
(4 \cdot 1-5 \cdot 8)\end{array}$ & $\begin{array}{l}13 \cdot 9 \\
(13 \cdot 2-14 \cdot 6)\end{array}$ & $\begin{array}{l}88 \cdot 9 \\
(88 \cdot 1-89 \cdot 8)\end{array}$ \\
\hline $2005-09$ & $\begin{array}{l}13 \cdot 1 \\
(11 \cdot 4-14 \cdot 8)\end{array}$ & $\begin{array}{l}23 \cdot 6 \\
(22 \cdot 1-25 \cdot 1)\end{array}$ & $\begin{array}{l}64 \cdot 2 \\
(63 \cdot 3-65 \cdot 2)\end{array}$ & $\begin{array}{l}63 \cdot 0 \\
(61 \cdot 9-64 \cdot 2)\end{array}$ & $\begin{array}{l}13 \cdot 0 \\
(11 \cdot 4-14 \cdot 6)\end{array}$ & $\begin{array}{c}7.9 \\
(6.9-8.9)\end{array}$ & $\begin{array}{l}16 \cdot 6 \\
(15 \cdot 9-17 \cdot 3)\end{array}$ & $\begin{array}{l}90 \cdot 3 \\
(89 \cdot 5-91 \cdot 0)\end{array}$ \\
\hline 2010-14 & $\begin{array}{l}14 \cdot 8 \\
(12 \cdot 8-16 \cdot 7)\end{array}$ & $\begin{array}{l}24 \cdot 8 \\
(23 \cdot 3-26 \cdot 3)\end{array}$ & $\begin{array}{l}64.9 \\
(64 \cdot 0-65 \cdot 8)\end{array}$ & $\begin{array}{l}64 \cdot 7 \\
(63 \cdot 5-65 \cdot 8)\end{array}$ & $\begin{array}{l}16 \cdot 6 \\
(14 \cdot 9-18 \cdot 3)\end{array}$ & $\begin{array}{c}9 \cdot 7 \\
(8 \cdot 7-10 \cdot 8)\end{array}$ & $\begin{array}{l}19 \cdot 5 \\
(18 \cdot 7-20 \cdot 2)\end{array}$ & $\begin{array}{l}91 \cdot 5 \\
(90 \cdot 9-92 \cdot 2)\end{array}$ \\
\hline \multicolumn{9}{|c|}{ Switzerland (ten registries) $\ddagger$} \\
\hline 2000-04 & $\begin{array}{l}16 \cdot 1 \\
(13 \cdot 6-18 \cdot 6)\end{array}$ & $\begin{array}{l}29 \cdot 2 \\
(26 \cdot 9-31 \cdot 4)\end{array}$ & $\begin{array}{l}62 \cdot 8 \\
(61 \cdot 3-64 \cdot 3)\end{array}$ & $\begin{array}{l}59 \cdot 5 \\
(57 \cdot 2-61 \cdot 8)\end{array}$ & $\begin{array}{l}12 \cdot 2 \\
(10 \cdot 3-14 \cdot 0)\end{array}$ & $\begin{array}{c}5 \cdot 6 \\
(4 \cdot 4-6 \cdot 9)\end{array}$ & $\begin{array}{l}14 \cdot 7 \\
(13 \cdot 8-15 \cdot 6)\end{array}$ & $\begin{array}{l}90 \cdot 7 \\
(88 \cdot 4-92 \cdot 9)\end{array}$ \\
\hline $2005-09$ & $\begin{array}{l}20 \cdot 6 \\
(17 \cdot 9-23 \cdot 2)\end{array}$ & $\begin{array}{c}31 \cdot 4 \\
(29 \cdot 0-33 \cdot 7)\end{array}$ & $\begin{array}{l}65 \cdot 1 \\
(63 \cdot 7-66 \cdot 6)\end{array}$ & $\begin{array}{l}65 \cdot 6 \\
(63 \cdot 5-67 \cdot 7)\end{array}$ & $\begin{array}{l}13 \cdot 4 \\
(11 \cdot 6-15 \cdot 3)\end{array}$ & $\begin{array}{c}7 \cdot 0 \\
(5 \cdot 8-8 \cdot 2)\end{array}$ & $\begin{array}{l}17 \cdot 3 \\
(16 \cdot 5-18 \cdot 2)\end{array}$ & $\begin{array}{l}92 \cdot 4 \\
(91 \cdot 3-93 \cdot 5)\end{array}$ \\
\hline 2010-14 & $\begin{array}{l}23 \cdot 9 \\
(21 \cdot 0-26 \cdot 9)\end{array}$ & $\begin{array}{l}32 \cdot 2 \\
(29 \cdot 9-34 \cdot 5)\end{array}$ & $\begin{array}{l}67 \cdot 3 \\
(65 \cdot 7-68 \cdot 9)\end{array}$ & $\begin{array}{l}67 \cdot 3 \\
(65 \cdot 0-69 \cdot 6)\end{array}$ & $\begin{array}{l}15 \cdot 4 \\
(13 \cdot 5-17 \cdot 4)\end{array}$ & $\begin{array}{c}9 \cdot 4 \\
(7 \cdot 9-10 \cdot 8)\end{array}$ & $\begin{array}{l}20 \cdot 4 \\
(19 \cdot 4-21 \cdot 4)\end{array}$ & $\begin{array}{l}93 \cdot 6 \\
(92 \cdot 4-94 \cdot 7)\end{array}$ \\
\hline \multicolumn{9}{|c|}{ UK (four registries)* } \\
\hline 2000-04 & $\begin{array}{l}11 \cdot 5 \\
(11 \cdot 1-11 \cdot 9)\end{array}$ & $\begin{array}{l}16 \cdot 2 \\
(15 \cdot 7-16 \cdot 6)\end{array}$ & $\begin{array}{l}52 \cdot 0 \\
(51 \cdot 6-52 \cdot 3)\end{array}$ & $\begin{array}{l}54 \cdot 6 \\
(54 \cdot 0-55 \cdot 1)\end{array}$ & $\begin{array}{c}7 \cdot 1 \\
(6 \cdot 6-7 \cdot 7)\end{array}$ & $\begin{array}{c}3 \cdot 7 \\
(3 \cdot 4-4 \cdot 0)\end{array}$ & $\begin{array}{c}8 \cdot 3 \\
(8 \cdot 1-8 \cdot 5)\end{array}$ & $\begin{array}{l}86 \cdot 4 \\
(85 \cdot 9-86 \cdot 8)\end{array}$ \\
\hline $2005-09$ & $\begin{array}{l}14 \cdot 0 \\
(13 \cdot 5-14 \cdot 4)\end{array}$ & $\begin{array}{l}19 \cdot 2 \\
(18 \cdot 7-19 \cdot 7)\end{array}$ & $\begin{array}{l}56 \cdot 5 \\
(56 \cdot 2-56 \cdot 9)\end{array}$ & $\begin{array}{l}58 \cdot 7 \\
(58 \cdot 2-59 \cdot 2)\end{array}$ & $\begin{array}{c}9.7 \\
(9 \cdot 1-10 \cdot 2)\end{array}$ & $\begin{array}{c}5 \cdot 2 \\
(4 \cdot 9-5 \cdot 5)\end{array}$ & $\begin{array}{l}10 \cdot 1 \\
(9 \cdot 9-10 \cdot 3)\end{array}$ & $\begin{array}{l}89 \cdot 2 \\
(88 \cdot 9-89 \cdot 6)\end{array}$ \\
\hline 2010-14 & $\begin{array}{l}15 \cdot 7 \\
(15 \cdot 3-16 \cdot 1)\end{array}$ & $\begin{array}{l}20 \cdot 7 \\
(20 \cdot 1-21 \cdot 2)\end{array}$ & $\begin{array}{l}60 \cdot 0 \\
(59 \cdot 7-60 \cdot 4)\end{array}$ & $\begin{array}{l}62 \cdot 5 \\
(62 \cdot 0-63.0)\end{array}$ & $\begin{array}{l}13 \cdot 0 \\
(12 \cdot 4-13 \cdot 6)\end{array}$ & $\begin{array}{c}6 \cdot 8 \\
(6 \cdot 5-7 \cdot 2)\end{array}$ & $\begin{array}{l}13 \cdot 3 \\
(13 \cdot 1-13 \cdot 5)\end{array}$ & $\begin{array}{l}90 \cdot 9 \\
(90 \cdot 6-91 \cdot 3)\end{array}$ \\
\hline \multicolumn{9}{|l|}{ Oceania } \\
\hline \multicolumn{9}{|c|}{ Australia (eight registries)* } \\
\hline 2000-04 & $\begin{array}{l}18 \cdot 0 \\
(16 \cdot 9-19 \cdot 2)\end{array}$ & $\begin{array}{l}27 \cdot 7 \\
(26 \cdot 7-28 \cdot 8)\end{array}$ & $\begin{array}{l}63 \cdot 7 \\
(63 \cdot 2-64 \cdot 3)\end{array}$ & $\begin{array}{l}64 \cdot 4 \\
(63 \cdot 5-65 \cdot 2)\end{array}$ & $\begin{array}{l}14 \cdot 2 \\
(13 \cdot 1-15 \cdot 3)\end{array}$ & $\begin{array}{c}7 \cdot 3 \\
(6 \cdot 7-8 \cdot 0)\end{array}$ & $\begin{array}{l}14 \cdot 8 \\
(14 \cdot 4-15 \cdot 2)\end{array}$ & $\begin{array}{l}92 \cdot 0 \\
(91 \cdot 7-92 \cdot 4)\end{array}$ \\
\hline $2005-09$ & $\begin{array}{l}19 \cdot 9 \\
(18 \cdot 7-21 \cdot 0)\end{array}$ & $\begin{array}{l}29 \cdot 8 \\
(28 \cdot 8-30 \cdot 9)\end{array}$ & $\begin{array}{l}68 \cdot 1 \\
(67 \cdot 6-68 \cdot 6)\end{array}$ & $\begin{array}{l}68 \cdot 6 \\
(67 \cdot 8-69 \cdot 5)\end{array}$ & $\begin{array}{l}17 \cdot 7 \\
(16 \cdot 6-18 \cdot 7)\end{array}$ & $\begin{array}{c}9 \cdot 1 \\
(8 \cdot 5-9 \cdot 8)\end{array}$ & $\begin{array}{l}17 \cdot 1 \\
(16 \cdot 7-17 \cdot 5)\end{array}$ & $\begin{array}{l}92 \cdot 5 \\
(92 \cdot 2-92 \cdot 8)\end{array}$ \\
\hline 2010-14 & $\begin{array}{l}23 \cdot 7 \\
(22 \cdot 4-25 \cdot 0)\end{array}$ & $\begin{array}{l}31 \cdot 8 \\
(30 \cdot 7-32 \cdot 9)\end{array}$ & $\begin{array}{l}70 \cdot 7 \\
(70 \cdot 1-71 \cdot 2)\end{array}$ & $\begin{array}{l}71 \cdot 0 \\
(70 \cdot 2-71 \cdot 9)\end{array}$ & $\begin{array}{l}19 \cdot 2 \\
(18 \cdot 1-20 \cdot 3)\end{array}$ & $\begin{array}{l}12 \cdot 0 \\
(11 \cdot 2-12 \cdot 8)\end{array}$ & $\begin{array}{l}19 \cdot 4 \\
(19 \cdot 0-19 \cdot 9)\end{array}$ & $\begin{array}{l}92 \cdot 9 \\
(92 \cdot 5-93 \cdot 2)\end{array}$ \\
\hline \multicolumn{9}{|c|}{ New Zealand* } \\
\hline 2000-04 & $\begin{array}{l}11 \cdot 5 \\
(9 \cdot 4-13 \cdot 6)\end{array}$ & $\begin{array}{l}24 \cdot 6 \\
(22 \cdot 4-26 \cdot 8)\end{array}$ & $\begin{array}{l}61 \cdot 4 \\
(60 \cdot 2-62 \cdot 7)\end{array}$ & $\begin{array}{l}60 \cdot 1 \\
(58 \cdot 0-62 \cdot 1)\end{array}$ & $\begin{array}{l}12 \cdot 4 \\
(9 \cdot 9-15 \cdot 0)\end{array}$ & $\begin{array}{c}7 \cdot 0 \\
(5 \cdot 5-8 \cdot 5)\end{array}$ & $\begin{array}{l}11 \cdot 4 \\
(10 \cdot 5-12 \cdot 2)\end{array}$ & $\begin{array}{l}90 \cdot 0 \\
(89 \cdot 1-90 \cdot 8)\end{array}$ \\
\hline 2005-09 & $\begin{array}{l}14 \cdot 5 \\
(12 \cdot 1-16 \cdot 8)\end{array}$ & $\begin{array}{l}24 \cdot 8 \\
(22 \cdot 5-27 \cdot 0)\end{array}$ & $\begin{array}{l}62 \cdot 8 \\
(61 \cdot 6-64 \cdot 0)\end{array}$ & $\begin{array}{l}63 \cdot 3 \\
(61 \cdot 4-65 \cdot 3)\end{array}$ & $\begin{array}{l}16 \cdot 8 \\
(14 \cdot 4-19 \cdot 1)\end{array}$ & $\begin{array}{c}7 \cdot 7 \\
(6 \cdot 3-9 \cdot 0)\end{array}$ & $\begin{array}{l}12 \cdot 4 \\
(11 \cdot 6-13 \cdot 3)\end{array}$ & $\begin{array}{l}91 \cdot 1 \\
(90 \cdot 3-91 \cdot 8)\end{array}$ \\
\hline 2010-14 & $\begin{array}{l}15 \cdot 3 \\
(13 \cdot 0-17 \cdot 7)\end{array}$ & $\begin{array}{l}25 \cdot 7 \\
(23 \cdot 5-27 \cdot 9)\end{array}$ & $\begin{array}{l}64 \cdot 0 \\
(62 \cdot 8-65 \cdot 1)\end{array}$ & $\begin{array}{l}66 \cdot 0 \\
(64 \cdot 1-67 \cdot 9)\end{array}$ & $\begin{array}{l}19 \cdot 0 \\
(16 \cdot 7-21 \cdot 4)\end{array}$ & $\begin{array}{c}8 \cdot 1 \\
(6 \cdot 7-9 \cdot 6)\end{array}$ & $\begin{array}{l}15 \cdot 3 \\
(14 \cdot 3-16 \cdot 2)\end{array}$ & $\begin{array}{l}91 \cdot 8 \\
(91 \cdot 1-92 \cdot 5)\end{array}$ \\
\hline $\begin{array}{l}\text { Some registrie } \\
\text { included in thi } \\
\text { coverage of th } \\
\text { censored alive } \\
\text { with unknown }\end{array}$ & $\begin{array}{l}\text { tributed data } \\
\text { le (see table } 7 \mathrm{f} \\
\text { ional populati } \\
\text { in } 5 \text { years of di } \\
\text { I status or regis }\end{array}$ & $\begin{array}{l}\text { hood cancers) } \\
\text { hildhood malic } \\
\text { or, if diagnose } \\
\text { ith incomplet }\end{array}$ & $\begin{array}{l}\text { a with } 100 \% \text { cov } \\
\text { ies only. \$Surviv } \\
010 \text { or later, bef } \\
\text {-ie, unknown }\end{array}$ & $\begin{array}{l}\text { stimate consic } \\
\text { Dec 31, 2014; } \\
\text { a of birth, unk }\end{array}$ & $\begin{array}{l}\text { al population } \\
\text { red less reliab } \\
\text { (2) registere } \\
\text { wn month o }\end{array}$ & $\begin{array}{l}\text { rvival estima } \\
\text { cause } 15 \% \text { or } \\
\text { y from a dea } \\
\text { of diagnosis }\end{array}$ & $\begin{array}{l}\text { arus, Greece, } \\
\text { ot age-stand } \\
\text { of patients w } \\
\text { ificate or at a } \\
\text { known year c }\end{array}$ & $\begin{array}{l}\text { lexico) are not } \\
\text { d. } \neq \text { Data with } 100 \% \\
\text { ) lost to follow-up } \\
\text { y; or (3) patients } \\
\text { known vital status }\end{array}$ \\
\hline
\end{tabular}

by $5-10 \%$ in Japan, Taiwan, and Turkey; Austria, Finland, France, Italy, Malta, and Sweden; and New Zealand.

Over the same period, 5-year survival increased by $10 \%$ or more in Canada; Israel and Korea; 16 European countries (Denmark, Estonia, Iceland, Ireland, Latvia, Lithuania, Norway, and the UK [northern Europe]; Portugal and Spain [southern Europe], Bulgaria, the Czech Republic, and Poland [eastern Europe]; and Germany, the 
Netherlands, and Switzerland [western Europe]); and Australia. The increase was about $20 \%$ or more in China, Korea, and Slovenia.

\section{Liver}

Results are available for 1178364 adults from 291 registries in 61 countries (tables 2, 4).

5 -year net survival was in the range 5-30\% throughout 2000-14 (appendix p 252). Estimates are often flagged as less reliable than for other solid tumours (table 6; appendix pp 139-151) because of the exclusion of higher proportions of DCO registrations (table 3; appendix pp 32-36).

For patients diagnosed during 2010-14, agestandardised 5-year net survival was in the range 20-29\% only in Korea, Singapore, and Taiwan; and Belgium and Italy. Survival was in the range $10-19 \%$ in 27 countries: Canada and the USA; Martinique; three Asian countries (China [east Asia]; and Kuwait and Turkey [west Asia]); 13 European countries (Iceland, Ireland, Latvia, Norway, Sweden, and the UK [northern Europe]; Portugal and Spain [southern Europe]; Poland [eastern Europe]; France, Germany, the Netherlands, and Switzerland [western Europe]); and Australia and New Zealand. 5-year survival was less than $10 \%$ in Denmark, Slovenia, Thailand, the Czech Republic, Russia, and Estonia.

In most countries, survival has changed very little during the 20-year period 1995-99 to 2000-14. It increased by $5-10 \%$ in Canada and the USA; Japan; eight European countries (Denmark, Ireland, and the UK [northern Europe]; Italy and Spain [southern Europe]; and France, the Netherlands, and Switzerland [western Europe]); and Australia and New Zealand. Survival increased by more than $10 \%$ in China, Korea, and Taiwan; and Norway, Portugal, and Sweden.

\section{Pancreas}

Results are available for 1229379 adults from 290 registries in 59 countries (tables 2, 4).

Age-standardised 5-year net survival estimates were generally in the range 5-15\% throughout 2000-14 (appendix p 253). Similarly to liver cancer, some estimates are less reliable (table 6; appendix pp 139-151) owing to the high proportion of DCO registrations (table 3; appendix pp 37-41).

For patients diagnosed during 2010-14, survival was high in Kuwait (23.6\%) and Malaysia (Penang; 19.0\%; table 6; appendix p 215). Survival was in the range 10-15\% in 15 countries: Canada and the USA; Martinique; China, Korea, and Turkey; eight European countries (Estonia, Ireland, Latvia, Norway, and Sweden [northern Europe]; Portugal [southern Europe]; and Belgium and Germany [western Europe]); and Australia. 5-year net survival ranged between $5 \%$ and $9 \%$ in 14 countries. Survival was very low in Russia (4.4\%).

Trends in 5-year survival between 2000-04 and 2010-14 were generally flat, but increases of $3-5 \%$ were seen in Canada and the USA; Korea and Singapore; 12 European countries (Denmark, Estonia, Ireland, Latvia, Norway, Sweden, and the UK [northern Europe]; Portugal [southern Europe]; the Czech Republic [eastern Europe]; and Belgium, the Netherlands, and Switzerland [western Europe]); and Australia (appendix p 234).

\section{Lung}

Results are available for 6051262 adults from 290 registries in 61 countries (tables 2, 4).

Age-standardised 5-year net survival was in the range $10-20 \%$ in most countries (table 6; appendix p 254). Most estimates in Central and South America were less reliable owing to the high proportion of DCO registrations excluded from analysis (table 6; appendix pp 152-164), although the proportion of DCOs has generally decreased worldwide (table 3; appendix pp 42-46).

For patients diagnosed during 2010-14, 5-year survival was high in Japan (32.9\%). It was in the range $20-30 \%$ in 12 countries: Mauritius; Canada and the USA; four Asian countries (China, Korea, and Taiwan [east Asia]; and Israel [west Asia]); and five European countries (Latvia, Iceland, and Sweden [northern Europe]; and Austria and Switzerland [western Europe]; table 6; appendix p 216). In most countries, however, survival was in the range 10-19\%: Martinique and Puerto Rico; five Asian countries (Malaysia [Penang] and Singapore [south Asia]; and Cyprus, Kuwait, and Turkey [west Asia]); 21 European countries (Denmark, Estonia, Finland, Ireland, Lithuania, Norway, and the UK [northern Europe]; Croatia, Italy, Malta, Portugal, Slovenia, and Spain [southern Europe] the Czech Republic, Poland, Russia, and Slovakia [eastern Europe]; and Belgium, France, Germany, and the Netherlands, [western Europe]); and Australia and New Zealand. Survival was less than $10 \%$ in Thailand, Brazil, Bulgaria, and India (table 6).

Lung cancer survival trends between 1995-99 and 2000-14 were generally flat, but survival increased by 5-10\% in 21 countries: Canada and the USA; Israel, Japan, and Taiwan; 15 European countries (Denmark, Estonia, Iceland, Ireland, Latvia, Norway, Sweden, and the UK [northern Europe]; Portugal and Slovenia [southern Europe]; and Austria, France, Germany, the Netherlands, and Switzerland [western Europe]); and Australia. Survival increased by more than $10 \%$ in China and Korea (table 6; appendix p 235).

\section{Melanoma of the skin}

Results are available for 1553109 adults from 281 registries in 59 countries (tables 2, 4).

Age-standardised 5-year net survival was in the range $60-90 \%$ in most countries (appendix p 255). Most estimates were considered reliable (table 6; appendix pp 152-164). For patients diagnosed during 2010-14, 5 -year survival estimates exceeded $90 \%$ in 11 countries: the USA; eight European countries (Denmark, Sweden, and the UK [northern Europe]; and Belgium, France, Germany, the Netherlands, and Switzerland [western 


\begin{tabular}{|c|c|c|c|c|c|c|c|c|c|c|}
\hline & \multicolumn{3}{|c|}{ Women's cancers } & \multirow[t]{2}{*}{ Prostate } & \multirow[t]{2}{*}{$\begin{array}{l}\text { Brain } \\
\text { (adults) }\end{array}$} & \multicolumn{2}{|c|}{$\begin{array}{l}\text { Haemopoietic malignancies } \\
\text { (adults) }\end{array}$} & \multicolumn{3}{|c|}{ Childhood malignancies } \\
\hline & Breast & Cervix & Ovary & & & Myeloid & Lymphoid & Brain & $\begin{array}{l}\text { Acute } \\
\text { lymphoblastic } \\
\text { leukaemia }\end{array}$ & Lymphoma \\
\hline \multicolumn{11}{|l|}{ Africa } \\
\hline \multicolumn{11}{|c|}{ Algeria (three registries) } \\
\hline 2000-04 & $\begin{array}{l}38 \cdot 95 \\
(29 \cdot 1-48 \cdot 7)\end{array}$ & $\begin{array}{l}61 \cdot 15 \\
(55 \cdot 5-66 \cdot 7)\end{array}$ & $\begin{array}{l}50 \cdot 7+\$ \\
(35 \cdot 6-65 \cdot 8)\end{array}$ & $\begin{array}{l}89.9+5 \\
(77 \cdot 8-100 \cdot 0)\end{array}$ & .. & $\begin{array}{l}21 \cdot 1+5 \\
(6 \cdot 0-36 \cdot 2)\end{array}$ & $\begin{array}{l}23 \cdot 3 \S \\
(13 \cdot 8-32 \cdot 8)\end{array}$ &.. & $\begin{array}{l}30 \cdot 9+5 \\
(10 \cdot 1-51 \cdot 6)\end{array}$ & $\begin{array}{c}6.0+5 \\
(0.0-15 \cdot 1)\end{array}$ \\
\hline 2005-09 & $\begin{array}{l}55 \cdot 65 \\
(47 \cdot 4-63 \cdot 8)\end{array}$ & $\begin{array}{l}70 \cdot 75 \\
(64 \cdot 5-77 \cdot 0)\end{array}$ & $\begin{array}{l}54 \cdot 35 \\
(44 \cdot 7-63 \cdot 8)\end{array}$ & $\begin{array}{l}50 \cdot 38 \\
(41 \cdot 0-59 \cdot 6)\end{array}$ & $\begin{array}{l}51 \cdot 95 \\
(43 \cdot 4-60 \cdot 4)\end{array}$ & $\begin{array}{l}17 \cdot 1+5 \\
(0 \cdot 1-34 \cdot 1)\end{array}$ & $\begin{array}{l}47 \cdot 85 \\
(39 \cdot 9-55 \cdot 8)\end{array}$ & $\begin{array}{l}43 \cdot 35 \\
(29 \cdot 4-57 \cdot 2)\end{array}$ & $\begin{array}{l}100 \cdot 0+5 \\
(100 \cdot 0-100 \cdot 0)\end{array}$ & $\begin{array}{c}78 \cdot 9+5 \\
(62 \cdot 5-95 \cdot 3)\end{array}$ \\
\hline 2010-14 & $\begin{array}{l}77.05 \\
(68.5-85.6)\end{array}$ & $\begin{array}{l}72 \cdot 45 \\
(66 \cdot 0-78 \cdot 7)\end{array}$ & $\begin{array}{l}66 \cdot 5 \S \\
(53 \cdot 5-79 \cdot 5)\end{array}$ & $\begin{array}{l}64 \cdot 15 \\
(56 \cdot 6-71 \cdot 6)\end{array}$ & $\begin{array}{l}46 \cdot 5 \S \\
(37 \cdot 5-55 \cdot 5)\end{array}$ & $\begin{array}{l}17 \cdot 95 \\
(5 \cdot 3-30 \cdot 6)\end{array}$ & $\begin{array}{l}59 \cdot 15 \\
(49 \cdot 9-68 \cdot 3)\end{array}$ & $\begin{array}{l}54 \cdot 1 \S \\
(39 \cdot 2-69 \cdot 0)\end{array}$ &. & $\begin{array}{l}77.5 \$ \\
(65.4-89.7)\end{array}$ \\
\hline \multicolumn{11}{|c|}{ Mali (Bamako) } \\
\hline 2000-04 & .. & .. & .. & .. & .. & .. & .. & .. & .. & .. \\
\hline 2005-09 & & .. &.. & & & & & &.. & \\
\hline 2010-14 & $\begin{array}{c}0.0+5 \\
(0.0-0.0)\end{array}$ &. &. & .. & & & .. & & .. & \\
\hline \multicolumn{11}{|l|}{ Mauritius* } \\
\hline 2000-04 & .. & .. & .. & .. & .. &.. & .. & .. & .. & .. \\
\hline 2005-09 & $\begin{array}{l}83 \cdot 6 \\
(75 \cdot 9-91 \cdot 3)\end{array}$ & $\begin{array}{l}80 \cdot 8 \\
(76 \cdot 0-85 \cdot 6)\end{array}$ & $\begin{array}{l}79 \cdot 7 \\
(69 \cdot 6-89 \cdot 8)\end{array}$ & $\begin{array}{l}61 \cdot 8 \\
(54 \cdot 1-69 \cdot 4)\end{array}$ &. &. & .. & .. &. & .. \\
\hline 2010-14 & &.$\cdot$ &.$\cdot$ & $\begin{array}{l}63 \cdot 5 \\
(54 \cdot 7-72 \cdot 4)\end{array}$ & $\begin{array}{l}43 \cdot 7 \dagger \\
(25 \cdot 5-61 \cdot 9)\end{array}$ & $\begin{array}{l}50 \cdot 1 \dagger \\
(34 \cdot 4-65 \cdot 8)\end{array}$ & $\begin{array}{l}65 \cdot 9 \\
(53 \cdot 4-78 \cdot 4)\end{array}$ & &.$\cdot$ & \\
\hline \multicolumn{11}{|c|}{ Morocco (Casablanca) } \\
\hline 2000-04 & & .. & .. & .. & .. & .. & .. & .. & .. & .. \\
\hline 2005-09 & $\begin{array}{l}86 \cdot 7 \S \\
(71 \cdot 7-100 \cdot 0)\end{array}$ &. &. &. &. &. & .. & .. &. & .. \\
\hline 2010-14 & $\begin{array}{l}99 \cdot 75 \\
(95 \cdot 8-100 \cdot 0)\end{array}$ &. &. &.. &. &. &. & .. &. &.. \\
\hline \multicolumn{11}{|c|}{ Nigeria (Ibadan) } \\
\hline 2000-04 &. & .. &.. &.$\cdot$ &.$\cdot$ &. & .. & .. &.$\cdot$ &.$\cdot$ \\
\hline 2005-09 & $\begin{array}{l}98.85 \\
(95 \cdot 6-100 \cdot 0)\end{array}$ & $\begin{array}{l}58 \cdot 65 \\
(46 \cdot 5-70 \cdot 7)\end{array}$ & $\begin{array}{l}59 \cdot 4+5 \\
(24 \cdot 9-93 \cdot 9)\end{array}$ & $\begin{array}{l}73 \cdot 95 \\
(50 \cdot 2-97 \cdot 6)\end{array}$ & $\begin{array}{l}88 \cdot 6+5 \\
(60 \cdot 4-100 \cdot 0)\end{array}$ & $\begin{array}{l}57 \cdot 4+5 \\
(29 \cdot 0-85 \cdot 8)\end{array}$ & $\begin{array}{l}86 \cdot 8 \S \\
(54 \cdot 9-100 \cdot 0)\end{array}$ & .. &.. & $\begin{array}{c}51 \cdot 0 \Omega \\
(31 \cdot 5-70 \cdot 6)\end{array}$ \\
\hline 2010-14 & $\begin{array}{l}97 \cdot 5 \$ \\
(89 \cdot 9-100 \cdot 0)\end{array}$ & $\begin{array}{l}49 \cdot 8 \$ \\
(36 \cdot 5-63 \cdot 1)\end{array}$ & $\begin{array}{l}49 \cdot 15 \\
(33 \cdot 8-64 \cdot 4)\end{array}$ & $\begin{array}{l}58 \cdot 75 \\
(40 \cdot 1-77 \cdot 2)\end{array}$ & $\begin{array}{l}54 \cdot 9+5 \\
(19 \cdot 7-90 \cdot 0)\end{array}$ & $\begin{array}{l}28 \cdot 4+5 \\
(3 \cdot 0-53 \cdot 7)\end{array}$ & $\begin{array}{l}56 \cdot 4 \Omega \\
(32 \cdot 2-80 \cdot 5)\end{array}$ &.. &. & $\begin{array}{c}6 \cdot 0 \S \\
(0 \cdot 0-13 \cdot 6)\end{array}$ \\
\hline \multicolumn{11}{|c|}{ South Africa (Eastern Cape) } \\
\hline 2000-04 & $\begin{array}{l}53 \cdot 0+\$ \\
(23 \cdot 4-82 \cdot 7)\end{array}$ & $\begin{array}{l}70 \cdot 78 \\
(56 \cdot 7-84 \cdot 7)\end{array}$ & $\begin{array}{l}82 \cdot 5+\$ \\
(42 \cdot 5-100 \cdot 0)\end{array}$ & $\begin{array}{l}77 \cdot 6+5 \\
(55 \cdot 0-100 \cdot 0)\end{array}$ &. &. & .. & .. &. & .. \\
\hline 2005-09 & $\begin{array}{l}32.05 \\
(23 \cdot 3-40 \cdot 7)\end{array}$ & $\begin{array}{l}40 \cdot 25 \\
(32 \cdot 2-48 \cdot 1)\end{array}$ & $\begin{array}{l}81 \cdot 0+5 \\
(58 \cdot 8-100 \cdot 0)\end{array}$ & $\begin{array}{l}38.65 \\
(25 \cdot 1-52 \cdot 0)\end{array}$ & .. &.. & $\begin{array}{l}29 \cdot 5+5 \\
(10 \cdot 8-48 \cdot 2)\end{array}$ &.. &.. & .. \\
\hline 2010-14 & $\begin{array}{l}40 \cdot 15 \\
(30 \cdot 7-49 \cdot 6)\end{array}$ & $\begin{array}{l}37 \cdot 15 \\
(31 \cdot 4-42 \cdot 9)\end{array}$ & $\begin{array}{l}67 \cdot 8+5 \\
(47 \cdot 4-88 \cdot 2)\end{array}$ & $\begin{array}{l}37 \cdot 85 \\
(25 \cdot 5-50 \cdot 1)\end{array}$ & $\begin{array}{l}51 \cdot 6+5 \\
(0 \cdot 6-100 \cdot 0)\end{array}$ & .. & $\begin{array}{l}47 \cdot 7+5 \\
(31 \cdot 1-64 \cdot 4)\end{array}$ &.. & .. &.. \\
\hline \multicolumn{11}{|c|}{ America (Central and South) } \\
\hline \multicolumn{11}{|c|}{ Argentina (five registries) $\ddagger$} \\
\hline $2000-04$ & $\begin{array}{l}82 \cdot 3 \\
(79 \cdot 4-85 \cdot 2)\end{array}$ & $\begin{array}{l}58 \cdot 3 \\
(52 \cdot 6-64 \cdot 0)\end{array}$ & $\begin{array}{l}40 \cdot 4 \\
(32 \cdot 4-48 \cdot 4)\end{array}$ & $\begin{array}{l}83 \cdot 5 \\
(78 \cdot 5-88 \cdot 6)\end{array}$ & $\begin{array}{l}29 \cdot 85 \\
(22 \cdot 6-37 \cdot 0)\end{array}$ & $\begin{array}{l}47 \cdot 9 \\
(39 \cdot 4-56 \cdot 3)\end{array}$ & $\begin{array}{l}54 \cdot 5 \\
(49 \cdot 1-59 \cdot 8)\end{array}$ & $\begin{array}{l}45 \cdot 1 \\
(41 \cdot 8-48 \cdot 3)\end{array}$ & $\begin{array}{l}65 \cdot 0 \\
(62 \cdot 7-67 \cdot 3)\end{array}$ & $\begin{array}{c}79 \cdot 9 \\
(76 \cdot 7-83 \cdot 0)\end{array}$ \\
\hline 2005-09 & $\begin{array}{l}82 \cdot 0 \\
(80 \cdot 4-83 \cdot 6)\end{array}$ & $\begin{array}{l}55 \cdot 6 \\
(52 \cdot 0-59 \cdot 1)\end{array}$ & $\begin{array}{l}43 \cdot 2 \\
(38 \cdot 6-47 \cdot 9)\end{array}$ & $\begin{array}{l}83 \cdot 6 \\
(81 \cdot 2-86 \cdot 0)\end{array}$ & $\begin{array}{l}27 \cdot 7 \S \\
(24 \cdot 4-31 \cdot 0)\end{array}$ & $\begin{array}{l}39 \cdot 5 \\
(34 \cdot 5-44 \cdot 6)\end{array}$ & $\begin{array}{l}48 \cdot 8 \\
(45 \cdot 9-51 \cdot 6)\end{array}$ & $\begin{array}{l}56 \cdot 1 \\
(52 \cdot 9-59 \cdot 3)\end{array}$ & $\begin{array}{l}72 \cdot 0 \\
(69 \cdot 7-74 \cdot 2)\end{array}$ & $\begin{array}{c}76 \cdot 9 \\
(73 \cdot 5-80 \cdot 3)\end{array}$ \\
\hline 2010-14 & $\begin{array}{l}84 \cdot 4 \\
(82 \cdot 6-86 \cdot 2)\end{array}$ & $\begin{array}{l}52 \cdot 7 \\
(48 \cdot 7-56 \cdot 7)\end{array}$ & $\begin{array}{l}38 \cdot 6 \\
(34 \cdot 3-42 \cdot 9)\end{array}$ & $\begin{array}{l}87 \cdot 6 \\
(84 \cdot 9-90 \cdot 4)\end{array}$ & $\begin{array}{l}30 \cdot 75 \\
(26 \cdot 8-34 \cdot 6)\end{array}$ & $\begin{array}{l}37 \cdot 4 \\
(32 \cdot 1-42 \cdot 6)\end{array}$ & $\begin{array}{l}48 \cdot 2 \\
(45 \cdot 0-51 \cdot 5)\end{array}$ & $\begin{array}{l}62.95 \\
(59 \cdot 4-66 \cdot 4)\end{array}$ & $\begin{array}{l}76 \cdot 15 \\
(73 \cdot 7-78 \cdot 4)\end{array}$ & $\begin{array}{c}83 \cdot 4 \\
(80 \cdot 1-86 \cdot 6)\end{array}$ \\
\hline \multicolumn{11}{|c|}{ Brazil (six registries) } \\
\hline 2000-04 & $\begin{array}{l}68.7 \S \\
(67.5-69.8)\end{array}$ & $\begin{array}{l}69 \cdot 3 \\
(66 \cdot 1-72 \cdot 6)\end{array}$ & $\begin{array}{l}42 \cdot 1 \\
(36 \cdot 0-48 \cdot 1)\end{array}$ & $\begin{array}{l}90 \cdot 0 \\
(87 \cdot 2-92 \cdot 8)\end{array}$ & $\begin{array}{l}31 \cdot 35 \\
(26 \cdot 2-36 \cdot 4)\end{array}$ & $\begin{array}{l}31 \cdot 1 \dagger \\
(7 \cdot 3-54 \cdot 9)\end{array}$ & $\begin{array}{l}48 \cdot 2 \\
(43 \cdot 7-52 \cdot 6)\end{array}$ & $\begin{array}{l}55 \cdot 7 \\
(44 \cdot 7-66 \cdot 6)\end{array}$ & $\begin{array}{l}67 \cdot 7 \\
(57 \cdot 8-77 \cdot 6)\end{array}$ & $\begin{array}{c}69 \cdot 2 \\
(57 \cdot 3-81 \cdot 1)\end{array}$ \\
\hline 2005-09 & $\begin{array}{l}76 \cdot 9 \S \\
(75 \cdot 7-78.0)\end{array}$ & $\begin{array}{l}63 \cdot 2 \\
(59 \cdot 9-66 \cdot 5)\end{array}$ & $\begin{array}{l}34 \cdot 1 \\
(29 \cdot 4-38 \cdot 9)\end{array}$ & $\begin{array}{l}92 \cdot 5 \\
(90 \cdot 2-94 \cdot 8)\end{array}$ & $\begin{array}{l}28 \cdot 25 \\
(24 \cdot 4-32 \cdot 1)\end{array}$ & $\begin{array}{l}41 \cdot 6 \\
(31 \cdot 4-51 \cdot 8)\end{array}$ & $\begin{array}{l}49 \cdot 4 \\
(45 \cdot 6-53 \cdot 2)\end{array}$ & $\begin{array}{l}34 \cdot 7 \\
(21 \cdot 9-47 \cdot 5)\end{array}$ & $\begin{array}{l}69 \cdot 8 \\
(60 \cdot 0-79 \cdot 6)\end{array}$ & $\begin{array}{c}86 \cdot 3 \\
(77 \cdot 1-95 \cdot 5)\end{array}$ \\
\hline \multirow[t]{2}{*}{ 2010-14 } & $\begin{array}{l}75 \cdot 2 \S \\
(73 \cdot 9-76 \cdot 5)\end{array}$ & $\begin{array}{l}60 \cdot 3 \\
(56 \cdot 3-64 \cdot 3)\end{array}$ & $\begin{array}{l}34 \cdot 9 \\
(29 \cdot 5-40 \cdot 3)\end{array}$ & $\begin{array}{l}91 \cdot 6 \\
(89 \cdot 1-94 \cdot 1)\end{array}$ & $\begin{array}{l}28 \cdot 15 \\
(23 \cdot 7-32 \cdot 6)\end{array}$ & $\begin{array}{l}39 \cdot 1 \\
(28 \cdot 7-49 \cdot 5)\end{array}$ & $\begin{array}{l}46 \cdot 2 \\
(42 \cdot 2-50 \cdot 2)\end{array}$ & $\begin{array}{l}28 \cdot 9 \\
(15 \cdot 8-41 \cdot 9)\end{array}$ & $\begin{array}{l}66 \cdot 0 \\
(53 \cdot 3-78 \cdot 8)\end{array}$ & $\begin{array}{l}88 \cdot 2 \\
(78 \cdot 2-98 \cdot 3)\end{array}$ \\
\hline & & & & & & & & & \multicolumn{2}{|c|}{ (Table 7 continues on next pag } \\
\hline
\end{tabular}




\begin{tabular}{|c|c|c|c|c|c|c|c|c|c|c|}
\hline & \multicolumn{3}{|c|}{ Women's cancers } & \multirow[t]{2}{*}{ Prostate } & \multirow[t]{2}{*}{$\begin{array}{l}\text { Brain } \\
\text { (adults) }\end{array}$} & \multicolumn{2}{|c|}{$\begin{array}{l}\text { Haemopoietic malignancies } \\
\text { (adults) }\end{array}$} & \multicolumn{3}{|c|}{ Childhood malignancies } \\
\hline & Breast & Cervix & Ovary & & & Myeloid & Lymphoid & Brain & $\begin{array}{l}\text { Acute } \\
\text { lymphoblastic } \\
\text { leukaemia }\end{array}$ & Lymphoma \\
\hline \multicolumn{11}{|c|}{ (Continued from previous page) } \\
\hline \multicolumn{11}{|c|}{ Chile (four registries) } \\
\hline $2000-04$ & $\begin{array}{l}74 \cdot 6 \\
(68 \cdot 2-81 \cdot 1)\end{array}$ & $\begin{array}{l}58 \cdot 2 \\
(52 \cdot 3-64 \cdot 0)\end{array}$ & $\begin{array}{l}25 \cdot 8 \\
(18 \cdot 5-33 \cdot 1)\end{array}$ & $\begin{array}{l}82 \cdot 6 \\
(77 \cdot 2-88 \cdot 0)\end{array}$ & $\begin{array}{l}12 \cdot 4 \dagger \\
(0 \cdot 0-26 \cdot 7)\end{array}$ & $\begin{array}{l}29 \cdot 1 \\
(19 \cdot 1-39 \cdot 1)\end{array}$ & $\begin{array}{l}28 \cdot 9 \\
(23 \cdot 4-34 \cdot 3)\end{array}$ & $\begin{array}{l}46 \cdot 7 \dagger \\
(22 \cdot 7-70 \cdot 7)\end{array}$ & $\begin{array}{l}76 \cdot 7 \\
(65 \cdot 6-87 \cdot 7)\end{array}$ & $\begin{array}{c}70 \cdot 1 \dagger \\
(43 \cdot 3-96 \cdot 9)\end{array}$ \\
\hline $2005-09$ & $\begin{array}{l}73 \cdot 5 \\
(68 \cdot 4-78 \cdot 6)\end{array}$ & $\begin{array}{l}57 \cdot 2 \\
(51 \cdot 6-62 \cdot 9)\end{array}$ & $\begin{array}{l}29 \cdot 0 \\
(23 \cdot 3-34 \cdot 7)\end{array}$ & $\begin{array}{l}84 \cdot 4 \\
(80 \cdot 7-88 \cdot 1)\end{array}$ & $\begin{array}{l}21 \cdot 8 \\
(14 \cdot 3-29 \cdot 3)\end{array}$ & $\begin{array}{l}25 \cdot 8 \\
(20 \cdot 8-30 \cdot 8)\end{array}$ & $\begin{array}{l}37 \cdot 1 \\
(33 \cdot 7-40 \cdot 5)\end{array}$ & $\begin{array}{l}56 \cdot 7 \dagger \\
(37 \cdot 0-76 \cdot 4)\end{array}$ & $\begin{array}{l}67 \cdot 4 \\
(57 \cdot 3-77 \cdot 4)\end{array}$ & $\begin{array}{l}69 \cdot 7 \dagger \\
(51 \cdot 4-88 \cdot 0)\end{array}$ \\
\hline 2010-14 & $\begin{array}{l}75 \cdot 5 \S \\
(69 \cdot 4-81 \cdot 5)\end{array}$ & $\begin{array}{l}56 \cdot 79 \\
(50 \cdot 0-63 \cdot 4)\end{array}$ & $\begin{array}{l}28.05 \\
(21 \cdot 3-34 \cdot 7)\end{array}$ & $\begin{array}{l}82.05 \\
(78.4-85 \cdot 5)\end{array}$ & $\begin{array}{l}24 \cdot 2 \\
(15 \cdot 2-33 \cdot 2)\end{array}$ & $\begin{array}{l}16 \cdot 5 \\
(11 \cdot 4-21 \cdot 6)\end{array}$ & $\begin{array}{l}32 \cdot 5 \\
(23 \cdot 8-41 \cdot 2)\end{array}$ &.. & $\begin{array}{l}63 \cdot 9 \\
(48 \cdot 5-79 \cdot 3)\end{array}$ &.. \\
\hline \multicolumn{11}{|c|}{ Colombia (four registries) } \\
\hline $2000-04$ & $\begin{array}{l}72 \cdot 3 \\
(68 \cdot 9-75 \cdot 7)\end{array}$ & $\begin{array}{l}56 \cdot 6 \$ \\
(53 \cdot 5-59 \cdot 8)\end{array}$ & $\begin{array}{l}33 \cdot 5 \\
(28 \cdot 0-39 \cdot 0)\end{array}$ & $\begin{array}{l}83 \cdot 6 \\
(80 \cdot 7-86 \cdot 5)\end{array}$ & $\begin{array}{l}23 \cdot 8 \\
(20 \cdot 1-27 \cdot 5)\end{array}$ & $\begin{array}{l}16 \cdot 3 \\
(11 \cdot 4-21 \cdot 2)\end{array}$ & $\begin{array}{l}37 \cdot 2 \\
(33 \cdot 4-40 \cdot 9)\end{array}$ & $\begin{array}{l}44 \cdot 95 \\
(32 \cdot 9-57 \cdot 0)\end{array}$ & $\begin{array}{l}52 \cdot 3 \\
(43 \cdot 5-61 \cdot 2)\end{array}$ & $\begin{array}{l}68 \cdot 45 \\
(55 \cdot 3-81 \cdot 6)\end{array}$ \\
\hline 2005-09 & $\begin{array}{l}79 \cdot 1 \\
(76 \cdot 1-82 \cdot 0)\end{array}$ & $\begin{array}{l}55 \cdot 4 \\
(52 \cdot 6-58 \cdot 1)\end{array}$ & $\begin{array}{l}35 \cdot 4 \\
(30 \cdot 3-40 \cdot 6)\end{array}$ & $\begin{array}{l}87 \cdot 8 \\
(85 \cdot 4-90 \cdot 3)\end{array}$ & $\begin{array}{l}27 \cdot 4 \\
(23 \cdot 5-31 \cdot 4)\end{array}$ & $\begin{array}{l}30 \cdot 7 \\
(25 \cdot 7-35 \cdot 7)\end{array}$ & $\begin{array}{l}42 \cdot 3 \\
(39 \cdot 2-45 \cdot 4)\end{array}$ & $\begin{array}{l}33 \cdot 95 \\
(23 \cdot 4-44 \cdot 5)\end{array}$ & $\begin{array}{l}57 \cdot 3 \\
(47 \cdot 8-66 \cdot 9)\end{array}$ & $\begin{array}{l}85 \cdot 05 \\
(76 \cdot 1-93 \cdot 9)\end{array}$ \\
\hline $2010-14$ & $\begin{array}{l}72 \cdot 15 \\
(69 \cdot 0-75 \cdot 2)\end{array}$ & $\begin{array}{l}49 \cdot 4 \varsigma \\
(46 \cdot 5-52 \cdot 3)\end{array}$ & $\begin{array}{l}33 \cdot 35 \\
(28 \cdot 2-38 \cdot 4)\end{array}$ & $\begin{array}{l}80 \cdot 35 \\
(77 \cdot 6-83 \cdot 1)\end{array}$ & $\begin{array}{l}20 \cdot 85 \\
(17 \cdot 1-24 \cdot 4)\end{array}$ & $\begin{array}{l}31 \cdot 85 \\
(26 \cdot 6-37 \cdot 1)\end{array}$ & $\begin{array}{l}40 \cdot 35 \\
(37 \cdot 0-43 \cdot 6)\end{array}$ & $\begin{array}{l}46.95 \\
(35 \cdot 9-57 \cdot 9)\end{array}$ & $\begin{array}{l}68 \cdot 9 \\
(51 \cdot 6-86 \cdot 3)\end{array}$ &.. \\
\hline \multicolumn{11}{|l|}{ Costa Rica* } \\
\hline 2000-04 & $\begin{array}{l}87 \cdot 0 \\
(83 \cdot 6-90 \cdot 3)\end{array}$ & $\begin{array}{l}84.95 \\
(81 \cdot 2-88 \cdot 6)\end{array}$ & $\begin{array}{l}54 \cdot 3 \\
(43 \cdot 7-64 \cdot 9)\end{array}$ & $\begin{array}{l}94 \cdot 0 \\
(92 \cdot 0-96 \cdot 1)\end{array}$ & $\begin{array}{l}30 \cdot 85 \\
(24 \cdot 9-36 \cdot 7)\end{array}$ & $\begin{array}{l}35 \cdot 0 \\
(25 \cdot 4-44 \cdot 6)\end{array}$ & $\begin{array}{l}50 \cdot 0 \\
(45 \cdot 2-54 \cdot 8)\end{array}$ & $\begin{array}{l}77 \cdot 1 \dagger \\
(55 \cdot 1-99 \cdot 0)\end{array}$ & $\begin{array}{l}97 \cdot 8 \dagger \\
(93 \cdot 2-100 \cdot 0)\end{array}$ &.. \\
\hline 2005-09 & $\begin{array}{l}86 \cdot 4 \\
(84 \cdot 0-88 \cdot 7)\end{array}$ & $\begin{array}{l}78 \cdot 38 \\
(75 \cdot 3-81 \cdot 3)\end{array}$ & $\begin{array}{l}47 \cdot 1 \\
(40 \cdot 5-53 \cdot 7)\end{array}$ & $\begin{array}{l}92 \cdot 6 \\
(90 \cdot 9-94 \cdot 2)\end{array}$ & $\begin{array}{l}18 \cdot 9 \Omega \\
(15 \cdot 1-22 \cdot 8)\end{array}$ & $\begin{array}{l}21 \cdot 2 \\
(15 \cdot 7-26 \cdot 7)\end{array}$ & $\begin{array}{l}50 \cdot 4 \\
(46 \cdot 9-53 \cdot 9)\end{array}$ & $\begin{array}{l}71 \cdot 2 \\
(61 \cdot 2-81 \cdot 3)\end{array}$ & $\begin{array}{l}87 \cdot 4 \\
(82 \cdot 8-91 \cdot 9)\end{array}$ & $\begin{array}{c}93 \cdot 0 \\
(86 \cdot 7-99 \cdot 3)\end{array}$ \\
\hline $2010-14$ & $\begin{array}{l}86 \cdot 7 \\
(84 \cdot 6-88 \cdot 9)\end{array}$ & $\begin{array}{l}78.05 \\
(74.8-81.2)\end{array}$ & $\begin{array}{l}56 \cdot 9 \\
(49 \cdot 1-64 \cdot 7)\end{array}$ & $\begin{array}{l}93 \cdot 2 \\
(91 \cdot 5-94 \cdot 9)\end{array}$ & $\begin{array}{l}21 \cdot 5 \S \\
(17 \cdot 5-25 \cdot 6)\end{array}$ & $\begin{array}{l}29 \cdot 4 \\
(23 \cdot 3-35 \cdot 4)\end{array}$ & $\begin{array}{l}52 \cdot 4 \\
(49 \cdot 1-55 \cdot 7)\end{array}$ & $\begin{array}{l}69 \cdot 8 \\
(57 \cdot 8-81 \cdot 8)\end{array}$ & $\begin{array}{l}80 \cdot 0 \\
(69 \cdot 3-90 \cdot 7)\end{array}$ & $\begin{array}{c}93 \cdot 5 \\
(86 \cdot 2-100 \cdot 0)\end{array}$ \\
\hline \multicolumn{11}{|l|}{ Cuba* } \\
\hline $2000-04$ & $\begin{array}{l}73 \cdot 7 \\
(72 \cdot 2-75 \cdot 1)\end{array}$ & $\begin{array}{l}64 \cdot 1 \\
(62 \cdot 3-65 \cdot 9)\end{array}$ & $\begin{array}{l}29 \cdot 5 \\
(26 \cdot 7-32 \cdot 3)\end{array}$ & $\begin{array}{l}26 \cdot 15 \\
(24 \cdot 6-27 \cdot 6)\end{array}$ &.. & .. & $\begin{array}{l}44.05 \\
(41 \cdot 6-46 \cdot 5)\end{array}$ & .. & .. & $\begin{array}{c}72 \cdot 45 \\
(67 \cdot 1-77 \cdot 8)\end{array}$ \\
\hline 2005-09 & $\begin{array}{l}81 \cdot 8 \\
(80 \cdot 5-83 \cdot 0)\end{array}$ & $\begin{array}{l}68 \cdot 8 \\
(67 \cdot 0-70 \cdot 7)\end{array}$ & $\begin{array}{l}38 \cdot 4 \\
(35 \cdot 6-41 \cdot 3)\end{array}$ & $\begin{array}{l}53 \cdot 85 \\
(52 \cdot 1-55 \cdot 6)\end{array}$ &.. &.. & $\begin{array}{l}49 \cdot 4 \varsigma \\
(46 \cdot 7-52 \cdot 0)\end{array}$ &.. &.. & $\begin{array}{c}77 \cdot 45 \\
(71 \cdot 5-83 \cdot 3)\end{array}$ \\
\hline 2010-14 & $\begin{array}{l}75 \cdot 1 \\
(73 \cdot 7-76 \cdot 5)\end{array}$ & $\begin{array}{l}72 \cdot 9 \\
(70 \cdot 5-75 \cdot 2)\end{array}$ & $\begin{array}{l}49 \cdot 3 \Omega \\
(45 \cdot 0-53 \cdot 5)\end{array}$ & $\begin{array}{l}71 \cdot 4 \Omega \\
(68 \cdot 9-74 \cdot 0)\end{array}$ &.. &.. & $\begin{array}{l}60 \cdot 15 \\
(56 \cdot 3-63 \cdot 8)\end{array}$ &.. &.. & $\begin{array}{c}78 \cdot 5 \S \\
(70 \cdot 5-86 \cdot 6)\end{array}$ \\
\hline \multicolumn{11}{|c|}{ Ecuador (five registries) } \\
\hline $2000-04$ & $\begin{array}{l}72 \cdot 1 \\
(67 \cdot 3-76 \cdot 9)\end{array}$ & $\begin{array}{l}47 \cdot 4 \\
(44 \cdot 1-50 \cdot 7)\end{array}$ & $\begin{array}{l}34 \cdot 5 \\
(26 \cdot 5-42 \cdot 5)\end{array}$ & $\begin{array}{l}85 \cdot 7 \\
(82 \cdot 0-89 \cdot 5)\end{array}$ & $\begin{array}{l}24 \cdot 2 \\
(19 \cdot 4-28 \cdot 9)\end{array}$ & $\begin{array}{l}20 \cdot 5 \\
(12 \cdot 6-28 \cdot 4)\end{array}$ & $\begin{array}{l}33 \cdot 1 \\
(29 \cdot 1-37 \cdot 0)\end{array}$ & $\begin{array}{l}48.05 \\
(35 \cdot 2-60.8)\end{array}$ & $\begin{array}{l}48 \cdot 35 \\
(40 \cdot 3-56 \cdot 4)\end{array}$ & $\begin{array}{c}73 \cdot 45 \\
(62 \cdot 7-84 \cdot 1)\end{array}$ \\
\hline $2005-09$ & $\begin{array}{l}75 \cdot 7 \\
(72 \cdot 7-78 \cdot 7)\end{array}$ & $\begin{array}{l}50 \cdot 4 \\
(47 \cdot 8-52 \cdot 9)\end{array}$ & $\begin{array}{l}38 \cdot 8 \\
(33 \cdot 1-44 \cdot 5)\end{array}$ & $\begin{array}{l}80 \cdot 7 \\
(78 \cdot 0-83 \cdot 5)\end{array}$ & $\begin{array}{l}20 \cdot 9 \\
(17 \cdot 6-24 \cdot 2)\end{array}$ & $\begin{array}{l}20 \cdot 3 \\
(15 \cdot 5-25 \cdot 0)\end{array}$ & $\begin{array}{l}39 \cdot 4 \\
(36 \cdot 7-42 \cdot 0)\end{array}$ & $\begin{array}{l}31 \cdot 2 \\
(20 \cdot 9-41 \cdot 5)\end{array}$ & $\begin{array}{l}51 \cdot 5 \\
(44 \cdot 9-58 \cdot 0)\end{array}$ & $\begin{array}{l}72 \cdot 2 \\
(63 \cdot 7-80 \cdot 7)\end{array}$ \\
\hline 2010-14 & $\begin{array}{l}75 \cdot 5 \\
(72 \cdot 4-78 \cdot 7)\end{array}$ & $\begin{array}{l}52 \cdot 0 \\
(49 \cdot 3-54 \cdot 7)\end{array}$ & $\begin{array}{l}37 \cdot 9 \\
(32 \cdot 1-43 \cdot 7)\end{array}$ & $\begin{array}{l}82 \cdot 2 \\
(79 \cdot 4-85 \cdot 0)\end{array}$ & $\begin{array}{l}25 \cdot 8 \\
(22 \cdot 0-29 \cdot 6)\end{array}$ & $\begin{array}{l}24 \cdot 4 \\
(18 \cdot 9-29 \cdot 9)\end{array}$ & $\begin{array}{l}40 \cdot 1 \\
(37 \cdot 4-42 \cdot 8)\end{array}$ & $\begin{array}{l}48 \cdot 2 \\
(35 \cdot 9-60 \cdot 5)\end{array}$ & $\begin{array}{l}49 \cdot 8 \\
(42 \cdot 7-56 \cdot 9)\end{array}$ & $\begin{array}{c}67 \cdot 3 \\
(57 \cdot 4-77 \cdot 2)\end{array}$ \\
\hline \multicolumn{11}{|l|}{ Guadeloupe* } \\
\hline $2000-04$ &.. &.. &.. &.. & .. &.. &.. &. &. &.. \\
\hline 2005-09 & $\begin{array}{l}69.85 \\
(60 \cdot 0-79 \cdot 7)\end{array}$ & $\begin{array}{l}20 \cdot 9+5 \\
(3 \cdot 3-38 \cdot 5)\end{array}$ & $\begin{array}{l}24 \cdot 2+5 \\
(8 \cdot 9-39 \cdot 5)\end{array}$ & $\begin{array}{l}82.85 \\
(78.2-87.5)\end{array}$ & $\begin{array}{c}0.2+5 \\
(0.0-0.6)\end{array}$ & $\begin{array}{l}37 \cdot 5+5 \\
(9 \cdot 8-65 \cdot 3)\end{array}$ & $\begin{array}{l}25 \cdot 7 \S \\
(17 \cdot 3-34 \cdot 0)\end{array}$ &.. &.. &.. \\
\hline $2010-14$ & $\begin{array}{l}50 \cdot 25 \\
(39 \cdot 6-60 \cdot 8)\end{array}$ & $\begin{array}{l}19 \cdot 45 \\
(9 \cdot 0-29 \cdot 9)\end{array}$ & $\begin{array}{l}29 \cdot 5+5 \\
(13 \cdot 8-45 \cdot 2)\end{array}$ & $\begin{array}{l}71 \cdot 45 \\
(65 \cdot 5-77 \cdot 2)\end{array}$ & $\begin{array}{c}9.6+5 \\
(0.0-19 \cdot 5)\end{array}$ & $\begin{array}{l}32 \cdot 38 \\
(21 \cdot 4-43 \cdot 2)\end{array}$ & $\begin{array}{l}36.05 \\
(27 \cdot 3-44 \cdot 6)\end{array}$ &.. &. &.. \\
\hline \multicolumn{11}{|l|}{ Martinique* } \\
\hline $2000-04$ & $\begin{array}{l}78.9 \\
(73.5-84 \cdot 3)\end{array}$ & $\begin{array}{l}69 \cdot 7 \\
(62 \cdot 5-76 \cdot 9)\end{array}$ & $\begin{array}{l}29 \cdot 5 \\
(17 \cdot 8-41 \cdot 1)\end{array}$ & $\begin{array}{l}93 \cdot 2 \\
(90 \cdot 5-95 \cdot 8)\end{array}$ & $\begin{array}{l}32 \cdot 6 \\
(22 \cdot 4-42 \cdot 8)\end{array}$ & $\begin{array}{l}46 \cdot 2 \\
(36 \cdot 8-55 \cdot 6)\end{array}$ & $\begin{array}{l}49 \cdot 8 \\
(44 \cdot 1-55 \cdot 5)\end{array}$ & .. & $\begin{array}{l}77 \cdot 5 \dagger \\
(55 \cdot 9-99 \cdot 2)\end{array}$ & .. \\
\hline $2005-09$ & $\begin{array}{l}87.8 \\
(83.5-92 \cdot 1)\end{array}$ & $\begin{array}{l}57 \cdot 6 \\
(48 \cdot 9-66 \cdot 3)\end{array}$ & $\begin{array}{l}34 \cdot 0 \\
(24 \cdot 6-43 \cdot 4)\end{array}$ & $\begin{array}{l}97 \cdot 8 \\
(95 \cdot 7-99 \cdot 9)\end{array}$ & $\begin{array}{l}28 \cdot 1 \\
(19 \cdot 9-36 \cdot 2)\end{array}$ & $\begin{array}{l}49 \cdot 7 \\
(41 \cdot 8-57 \cdot 6)\end{array}$ & $\begin{array}{l}48 \cdot 7 \\
(43 \cdot 9-53 \cdot 6)\end{array}$ &. &.. &.. \\
\hline 2010-14 & $\begin{array}{l}89 \cdot 8 \\
(84 \cdot 5-95 \cdot 1)\end{array}$ & $\begin{array}{l}57 \cdot 5 \\
(46 \cdot 2-68 \cdot 8)\end{array}$ & $\begin{array}{l}35 \cdot 75 \\
(23 \cdot 4-48 \cdot 0)\end{array}$ & $\begin{array}{l}97 \cdot 9 \\
(95 \cdot 1-100 \cdot 0)\end{array}$ & $\begin{array}{l}35 \cdot 8 \\
(26 \cdot 4-45 \cdot 3)\end{array}$ & $\begin{array}{l}47 \cdot 9 \\
(39 \cdot 4-56 \cdot 5)\end{array}$ & $\begin{array}{l}49 \cdot 4 \\
(43 \cdot 3-55 \cdot 4)\end{array}$ &.. & .. &.. \\
\hline
\end{tabular}




\begin{tabular}{|c|c|c|c|c|c|c|c|c|c|c|}
\hline & \multicolumn{3}{|c|}{ Women's cancers } & \multirow[t]{2}{*}{ Prostate } & \multirow[t]{2}{*}{$\begin{array}{l}\text { Brain } \\
\text { (adults) }\end{array}$} & \multicolumn{2}{|c|}{$\begin{array}{l}\text { Haemopoietic malignancies } \\
\text { (adults) }\end{array}$} & \multicolumn{3}{|c|}{ Childhood malignancies } \\
\hline & Breast & Cervix & Ovary & & & Myeloid & Lymphoid & Brain & $\begin{array}{l}\text { Acute } \\
\text { lymphoblastic } \\
\text { leukaemia }\end{array}$ & Lymphoma \\
\hline \multicolumn{11}{|c|}{ (Continued from previous page) } \\
\hline \multicolumn{11}{|c|}{ Mexico (childhood) $\ddagger$} \\
\hline $2000-04$ &.$\cdot$ & .. &.. &.$\cdot$ &.. &.$\cdot$ & .. & .. & .. & .. \\
\hline 2005-09 &. & .. &.. &.$\cdot$ &.. &.$\cdot$ & .. & $\begin{array}{l}41 \cdot 2 \\
(35 \cdot 1-47 \cdot 4)\end{array}$ & $\begin{array}{l}52 \cdot 5 \\
(49 \cdot 8-55 \cdot 2)\end{array}$ & $\begin{array}{c}75 \cdot 15 \\
(70 \cdot 7-79 \cdot 5)\end{array}$ \\
\hline 2010-14 &.$\cdot$ & .. &.. &.$\cdot$ &.$\cdot$ &.$\cdot$ & .. & $\begin{array}{l}36 \cdot 5 \\
(30 \cdot 0-43 \cdot 0)\end{array}$ & $\begin{array}{l}52 \cdot 7 \\
(49 \cdot 4-56 \cdot 1)\end{array}$ & $\begin{array}{l}72 \cdot 0 \$ \\
(66 \cdot 4-77 \cdot 7)\end{array}$ \\
\hline \multicolumn{11}{|l|}{ Peru (Lima) } \\
\hline $2000-04$ &.$\cdot$ & .. &.. &.$\cdot$ &.. & .. & .. & .. &.$\cdot$ & .. \\
\hline $2005-09$ &.. & .. &.. & .. & .. &.. & .. & .. & .. & .. \\
\hline 2010-14 & $\begin{array}{l}84 \cdot 0 \\
(81 \cdot 4-86 \cdot 7)\end{array}$ & $\begin{array}{l}57 \cdot 2 \\
(54 \cdot 8-59 \cdot 6)\end{array}$ &.$\cdot$ & .. &.$\cdot$ & $\begin{array}{l}36 \cdot 35 \\
(29 \cdot 5-43 \cdot 0)\end{array}$ & $\begin{array}{l}46 \cdot 25 \\
(42 \cdot 9-49 \cdot 6)\end{array}$ & .. & $\begin{array}{l}60 \cdot 4 \\
(53 \cdot 4-67 \cdot 3)\end{array}$ & $\begin{array}{c}73 \cdot 45 \\
(64 \cdot 8-82 \cdot 1)\end{array}$ \\
\hline \multicolumn{11}{|l|}{ Puerto Rico* } \\
\hline $2000-04$ & $\begin{array}{l}83 \cdot 4 \\
(81 \cdot 9-84 \cdot 9)\end{array}$ & $\begin{array}{l}60 \cdot 6 \\
(56 \cdot 9-64 \cdot 4)\end{array}$ & $\begin{array}{l}34 \cdot 9 \\
(30 \cdot 8-39 \cdot 0)\end{array}$ & $\begin{array}{l}98 \cdot 7 \\
(97 \cdot 6-99 \cdot 7)\end{array}$ & $\begin{array}{l}31 \cdot 75 \\
(28 \cdot 0-35 \cdot 4)\end{array}$ & $\begin{array}{l}25 \cdot 65 \\
(21 \cdot 4-29 \cdot 8)\end{array}$ & $\begin{array}{l}49 \cdot 1 \\
(46 \cdot 8-51 \cdot 4)\end{array}$ & $\begin{array}{l}71 \cdot 2 \\
(62 \cdot 1-80 \cdot 3)\end{array}$ & $\begin{array}{l}79 \cdot 3 \\
(70 \cdot 2-88 \cdot 4)\end{array}$ & $\begin{array}{c}94.5 \\
(90 \cdot 1-98.8)\end{array}$ \\
\hline 2005-09 & $\begin{array}{l}83 \cdot 0 \\
(81 \cdot 6-84 \cdot 4)\end{array}$ & $\begin{array}{l}58 \cdot 8 \\
(55 \cdot 3-62 \cdot 4)\end{array}$ & $\begin{array}{l}37 \cdot 2 \\
(33 \cdot 4-41 \cdot 0)\end{array}$ & $\begin{array}{l}99 \cdot 0 \\
(98 \cdot 1-99 \cdot 9)\end{array}$ & $\begin{array}{l}34 \cdot 8 \\
(31 \cdot 5-38 \cdot 0)\end{array}$ & $\begin{array}{l}38 \cdot 8 \\
(35 \cdot 6-42 \cdot 0)\end{array}$ & $\begin{array}{l}53 \cdot 5 \\
(51 \cdot 6-55 \cdot 4)\end{array}$ & $\begin{array}{l}76 \cdot 1 \\
(67 \cdot 6-84 \cdot 6)\end{array}$ & $\begin{array}{l}86 \cdot 2 \\
(78 \cdot 1-94 \cdot 4)\end{array}$ & $\begin{array}{c}90 \cdot 9 \\
(83 \cdot 1-98 \cdot 7)\end{array}$ \\
\hline 2010-14 & $\begin{array}{l}84 \cdot 1 \\
(82 \cdot 0-86 \cdot 3)\end{array}$ & $\begin{array}{l}63 \cdot 5 \\
(57 \cdot 9-69 \cdot 1)\end{array}$ & $\begin{array}{l}37 \cdot 3 \\
(32 \cdot 0-42 \cdot 6)\end{array}$ & $\begin{array}{l}98 \cdot 4 \\
(97 \cdot 0-99 \cdot 8)\end{array}$ & $\begin{array}{l}36 \cdot 3 \\
(31 \cdot 2-41 \cdot 4)\end{array}$ & $\begin{array}{l}44.9 \\
(39 \cdot 6-50 \cdot 1)\end{array}$ & $\begin{array}{l}60 \cdot 3 \\
(57 \cdot 2-63 \cdot 4)\end{array}$ & $\begin{array}{l}70 \cdot 5 \\
(56 \cdot 3-84 \cdot 7)\end{array}$ & $\begin{array}{l}93 \cdot 1 \\
(83 \cdot 6-100 \cdot 0)\end{array}$ & $\begin{array}{c}96 \cdot 3 \\
(89 \cdot 9-100 \cdot 0)\end{array}$ \\
\hline \multicolumn{11}{|l|}{ Uruguay* } \\
\hline $2000-04$ &.$\cdot$ &.$\cdot$ &.$\cdot$ &.$\cdot$ &.$\cdot$ &.$\cdot$ & .. & .. &.$\cdot$ &.$\cdot$ \\
\hline 2005-09 &. & $\begin{array}{l}55 \cdot 7 \\
(51 \cdot 6-59 \cdot 8)\end{array}$ & $\begin{array}{l}37 \cdot 4 \\
(31 \cdot 9-42 \cdot 8)\end{array}$ & $\begin{array}{l}84 \cdot 7 \\
(82 \cdot 1-87 \cdot 3)\end{array}$ &.. &.$\cdot$ & .. & .. &.$\cdot$ &. \\
\hline 2010-14 &. & $\begin{array}{l}56 \cdot 5 \\
(51 \cdot 8-61 \cdot 1)\end{array}$ & $\begin{array}{l}37 \cdot 45 \\
(31 \cdot 4-43 \cdot 4)\end{array}$ & $\begin{array}{l}86 \cdot 5 \\
(83 \cdot 7-89 \cdot 3)\end{array}$ &.. &. & .. & .. & .. & .. \\
\hline \multicolumn{11}{|c|}{ America (North) } \\
\hline \multicolumn{11}{|c|}{ Canada (nine registries) } \\
\hline $2000-04$ & $\begin{array}{l}85 \cdot 9 \\
(85 \cdot 5-86 \cdot 4)\end{array}$ & $\begin{array}{l}67 \cdot 9 \\
(66 \cdot 4-69 \cdot 5)\end{array}$ & $\begin{array}{l}37 \cdot 6 \\
(36 \cdot 6-38 \cdot 6)\end{array}$ & $\begin{array}{l}93 \cdot 0 \\
(92 \cdot 6-93 \cdot 3)\end{array}$ & $\begin{array}{l}24 \cdot 7 \\
(23 \cdot 4-26 \cdot 0)\end{array}$ & $\begin{array}{l}47 \cdot 5 \\
(46 \cdot 1-48 \cdot 8)\end{array}$ & $\begin{array}{l}60 \cdot 1 \\
(59 \cdot 5-60 \cdot 7)\end{array}$ & $\begin{array}{l}73 \cdot 3 \\
(68 \cdot 2-78 \cdot 4)\end{array}$ & $\begin{array}{l}91 \cdot 0 \\
(88 \cdot 8-93 \cdot 3)\end{array}$ & $\begin{array}{c}89 \cdot 4 \\
(85 \cdot 9-92 \cdot 9)\end{array}$ \\
\hline $2005-09$ & $\begin{array}{l}87 \cdot 6 \\
(87 \cdot 2-88 \cdot 0)\end{array}$ & $\begin{array}{l}66 \cdot 9 \\
(65 \cdot 3-68 \cdot 5)\end{array}$ & $\begin{array}{l}41 \cdot 0 \\
(40 \cdot 0-42 \cdot 0)\end{array}$ & $\begin{array}{l}94 \cdot 2 \\
(93 \cdot 9-94 \cdot 5)\end{array}$ & $\begin{array}{l}29 \cdot 8 \\
(28 \cdot 5-31 \cdot 1)\end{array}$ & $\begin{array}{l}49 \cdot 2 \\
(47 \cdot 9-50 \cdot 5)\end{array}$ & $\begin{array}{l}66 \cdot 0 \\
(65 \cdot 4-66 \cdot 5)\end{array}$ & $\begin{array}{l}72 \cdot 5 \\
(67 \cdot 7-77 \cdot 2)\end{array}$ & $\begin{array}{l}92 \cdot 1 \\
(90 \cdot 0-94 \cdot 3)\end{array}$ & $\begin{array}{c}88 \cdot 5 \\
(84 \cdot 4-92 \cdot 5)\end{array}$ \\
\hline 2010-14 & $\begin{array}{l}88 \cdot 2 \\
(87 \cdot 8-88.6)\end{array}$ & $\begin{array}{l}66 \cdot 6 \\
(65 \cdot 1-68 \cdot 1)\end{array}$ & $\begin{array}{l}40 \cdot 9 \\
(39 \cdot 9-41 \cdot 8)\end{array}$ & $\begin{array}{l}93 \cdot 6 \\
(93 \cdot 3-94 \cdot 0)\end{array}$ & $\begin{array}{l}29 \cdot 9 \\
(28 \cdot 6-31 \cdot 1)\end{array}$ & $\begin{array}{l}50 \cdot 4 \\
(49 \cdot 2-51 \cdot 6)\end{array}$ & $\begin{array}{l}68 \cdot 6 \\
(68 \cdot 1-69 \cdot 1)\end{array}$ & $\begin{array}{l}72 \cdot 7 \\
(68 \cdot 0-77 \cdot 4)\end{array}$ & $\begin{array}{l}92 \cdot 6 \\
(90 \cdot 7-94 \cdot 6)\end{array}$ & $\begin{array}{c}92 \cdot 3 \\
(89 \cdot 1-95 \cdot 6)\end{array}$ \\
\hline \multicolumn{11}{|c|}{ USA (48 registries) } \\
\hline 2000-04 & $\begin{array}{l}88.9 \\
(88.7-89.0)\end{array}$ & $\begin{array}{l}64 \cdot 3 \\
(63 \cdot 7-64 \cdot 8)\end{array}$ & $\begin{array}{l}40 \cdot 4 \\
(40 \cdot 0-40 \cdot 7)\end{array}$ & $\begin{array}{l}97 \cdot 5 \\
(97 \cdot 3-97 \cdot 6)\end{array}$ & $\begin{array}{l}26 \cdot 8 \\
(26 \cdot 5-27 \cdot 1)\end{array}$ & $\begin{array}{l}41 \cdot 0 \\
(40 \cdot 7-41 \cdot 3)\end{array}$ & $\begin{array}{l}61 \cdot 2 \\
(61 \cdot 0-61 \cdot 4)\end{array}$ & $\begin{array}{l}72 \cdot 1 \\
(71 \cdot 1-73 \cdot 2)\end{array}$ & $\begin{array}{l}86 \cdot 7 \\
(85 \cdot 8-87 \cdot 5)\end{array}$ & $\begin{array}{c}88 \cdot 5 \\
(87 \cdot 4-89 \cdot 6)\end{array}$ \\
\hline 2005-09 & $\begin{array}{l}89 \cdot 8 \\
(89 \cdot 6-89 \cdot 9)\end{array}$ & $\begin{array}{l}63 \cdot 0 \\
(62 \cdot 5-63 \cdot 5)\end{array}$ & $\begin{array}{l}42 \cdot 0 \\
(41 \cdot 7-42 \cdot 4)\end{array}$ & $\begin{array}{l}98 \cdot 1 \\
(98.0-98 \cdot 2)\end{array}$ & $\begin{array}{l}35 \cdot 1 \\
(34 \cdot 8-35 \cdot 4)\end{array}$ & $\begin{array}{l}45 \cdot 9 \\
(45 \cdot 6-46 \cdot 2)\end{array}$ & $\begin{array}{l}66 \cdot 1 \\
(66 \cdot 0-66 \cdot 3)\end{array}$ & $\begin{array}{l}76 \cdot 8 \\
(75 \cdot 9-77 \cdot 7)\end{array}$ & $\begin{array}{l}88 \cdot 1 \\
(87 \cdot 3-88 \cdot 9)\end{array}$ & $\begin{array}{c}90 \cdot 2 \\
(89 \cdot 2-91 \cdot 3)\end{array}$ \\
\hline 2010-14 & $\begin{array}{l}90 \cdot 2 \\
(90 \cdot 1-90 \cdot 4)\end{array}$ & $\begin{array}{l}62 \cdot 6 \\
(62 \cdot 0-63 \cdot 1)\end{array}$ & $\begin{array}{l}43 \cdot 4 \\
(43 \cdot 1-43 \cdot 8)\end{array}$ & $\begin{array}{l}97 \cdot 4 \\
(97 \cdot 3-97 \cdot 5)\end{array}$ & $\begin{array}{l}36 \cdot 5 \\
(36 \cdot 1-36 \cdot 8)\end{array}$ & $\begin{array}{l}46 \cdot 7 \\
(46 \cdot 4-47 \cdot 0)\end{array}$ & $\begin{array}{l}68 \cdot 1 \\
(67 \cdot 9-68 \cdot 3)\end{array}$ & $\begin{array}{l}78 \cdot 2 \\
(77 \cdot 3-79 \cdot 2)\end{array}$ & $\begin{array}{l}89.5 \\
(88 \cdot 8-90 \cdot 3)\end{array}$ & $\begin{array}{c}94 \cdot 3 \\
(93 \cdot 6-95 \cdot 1)\end{array}$ \\
\hline \multicolumn{11}{|l|}{ Asia } \\
\hline \multicolumn{11}{|c|}{ China (21 registries) } \\
\hline $2000-04$ & $\begin{array}{l}75 \cdot 9 \\
(70 \cdot 9-80 \cdot 9)\end{array}$ & $\begin{array}{l}53 \cdot 3 \\
(48 \cdot 1-58 \cdot 5)\end{array}$ & $\begin{array}{l}42 \cdot 4 \\
(38 \cdot 2-46 \cdot 6)\end{array}$ & $\begin{array}{l}57 \cdot 7 \\
(52 \cdot 3-63 \cdot 0)\end{array}$ & $\begin{array}{l}22 \cdot 7 \\
(20 \cdot 5-25 \cdot 0)\end{array}$ & $\begin{array}{l}18 \cdot 6 \\
(15 \cdot 8-21 \cdot 5)\end{array}$ & $\begin{array}{l}33 \cdot 9 \\
(31 \cdot 6-36 \cdot 2)\end{array}$ & $\begin{array}{l}32 \cdot 7 \\
(21 \cdot 0-44 \cdot 4)\end{array}$ & $\begin{array}{l}61 \cdot 8 \\
(46 \cdot 5-77 \cdot 2)\end{array}$ & $\begin{array}{c}44 \cdot 2 \dagger \\
(29 \cdot 7-58 \cdot 8)\end{array}$ \\
\hline 2005-09 & $\begin{array}{l}80 \cdot 4 \\
(79 \cdot 3-81 \cdot 5)\end{array}$ & $\begin{array}{l}63 \cdot 0 \\
(61 \cdot 2-64 \cdot 9)\end{array}$ & $\begin{array}{l}40 \cdot 6 \\
(38 \cdot 8-42 \cdot 5)\end{array}$ & $\begin{array}{l}62 \cdot 5 \\
(59 \cdot 9-65 \cdot 1)\end{array}$ & $\begin{array}{l}26 \cdot 4 \\
(25 \cdot 2-27 \cdot 7)\end{array}$ & $\begin{array}{l}20 \cdot 1 \\
(18 \cdot 7-21 \cdot 4)\end{array}$ & $\begin{array}{l}35 \cdot 4 \\
(34 \cdot 2-36 \cdot 6)\end{array}$ & $\begin{array}{l}39 \cdot 1 \\
(31 \cdot 2-47 \cdot 0)\end{array}$ & $\begin{array}{l}53 \cdot 5 \\
(44 \cdot 6-62 \cdot 4)\end{array}$ & $\begin{array}{c}52 \cdot 3 \\
(39 \cdot 5-65 \cdot 0)\end{array}$ \\
\hline \multirow[t]{2}{*}{ 2010-14 } & $\begin{array}{l}83 \cdot 2 \\
(82 \cdot 1-84 \cdot 3)\end{array}$ & $\begin{array}{l}67 \cdot 6 \\
(65 \cdot 8-69 \cdot 5)\end{array}$ & $\begin{array}{l}41 \cdot 8 \\
(39 \cdot 8-43 \cdot 7)\end{array}$ & $\begin{array}{l}69 \cdot 2 \\
(66 \cdot 4-72 \cdot 0)\end{array}$ & $\begin{array}{l}32 \cdot 0 \\
(30 \cdot 6-33 \cdot 5)\end{array}$ & $\begin{array}{l}24 \cdot 8 \\
(23 \cdot 2-26 \cdot 4)\end{array}$ & $\begin{array}{l}38 \cdot 3 \\
(37 \cdot 0-39 \cdot 5)\end{array}$ & $\begin{array}{l}41 \cdot 1 \\
(32 \cdot 0-50 \cdot 1)\end{array}$ & $\begin{array}{l}57 \cdot 7 \\
(46 \cdot 6-68 \cdot 7)\end{array}$ & $\begin{array}{c}61 \cdot 1 \\
(47 \cdot 5-74 \cdot 8)\end{array}$ \\
\hline & & & & & & & & & \multicolumn{2}{|c|}{ (Table 7 continues on next page } \\
\hline
\end{tabular}




\begin{tabular}{|c|c|c|c|c|c|c|c|c|c|c|}
\hline & \multicolumn{3}{|c|}{ Women's cancers } & \multirow[t]{2}{*}{ Prostate } & \multirow[t]{2}{*}{$\begin{array}{l}\text { Brain } \\
\text { (adults) }\end{array}$} & \multicolumn{2}{|c|}{$\begin{array}{l}\text { Haemopoietic malignancies } \\
\text { (adults) }\end{array}$} & \multicolumn{3}{|c|}{ Childhood malignancies } \\
\hline & Breast & Cervix & Ovary & & & Myeloid & Lymphoid & Brain & $\begin{array}{l}\text { Acute } \\
\text { lymphoblastic } \\
\text { leukaemia }\end{array}$ & Lymphoma \\
\hline \multicolumn{11}{|c|}{ (Continued from previous page) } \\
\hline \multicolumn{11}{|l|}{ Cyprus* } \\
\hline 2000-04 & $\begin{array}{l}89 \cdot 3 \S \\
(80 \cdot 8-97 \cdot 8)\end{array}$ & $\begin{array}{l}60 \cdot 4+5 \\
(41 \cdot 0-79 \cdot 8)\end{array}$ & $\begin{array}{l}42 \cdot 6+5 \\
(24 \cdot 6-60 \cdot 6)\end{array}$ & $\begin{array}{l}91 \cdot 0 \Omega \\
(84 \cdot 0-97 \cdot 9)\end{array}$ & $\begin{array}{l}32 \cdot 1+5 \\
(13 \cdot 4-50 \cdot 8)\end{array}$ & $\begin{array}{l}60 \cdot 0+5 \\
(39 \cdot 1-80 \cdot 9)\end{array}$ & $\begin{array}{l}61 \cdot 4 \Omega \\
(52 \cdot 2-70 \cdot 6)\end{array}$ &. & & .. \\
\hline 2005-09 & $\begin{array}{l}92 \cdot 05 \\
(88 \cdot 7-95 \cdot 4)\end{array}$ & $\begin{array}{l}66 \cdot 9 \Omega \\
(60 \cdot 0-73 \cdot 8)\end{array}$ & $\begin{array}{l}46 \cdot 25 \\
(39 \cdot 9-52 \cdot 4)\end{array}$ & $\begin{array}{l}98 \cdot 35 \\
(95 \cdot 4-100 \cdot 0)\end{array}$ & $\begin{array}{l}24 \cdot 6 \$ \\
(18 \cdot 6-30 \cdot 7)\end{array}$ & $\begin{array}{l}39 \cdot 85 \\
(33 \cdot 3-46 \cdot 2)\end{array}$ & $\begin{array}{l}69 \cdot 45 \\
(65 \cdot 1-73 \cdot 7)\end{array}$ & & $\begin{array}{l}85 \cdot 2+5 \\
(72 \cdot 1-98 \cdot 3)\end{array}$ & $\begin{array}{l}100 \cdot 0+5 \\
(86 \cdot 3-100 \cdot 0)\end{array}$ \\
\hline 2010-14 & $\begin{array}{l}92 \cdot 8 \$ \\
(89 \cdot 7-95 \cdot 9)\end{array}$ & $\begin{array}{l}73 \cdot 38 \\
(65 \cdot 1-81 \cdot 6)\end{array}$ & $\begin{array}{l}46 \cdot 45 \\
(40 \cdot 0-52 \cdot 7)\end{array}$ & $\begin{array}{l}99 \cdot 2 \Omega \\
(96 \cdot 4-100 \cdot 0)\end{array}$ & $\begin{array}{l}25 \cdot 25 \\
(19 \cdot 5-31 \cdot 0)\end{array}$ & $\begin{array}{l}36 \cdot 0 \Omega \\
(30 \cdot 2-41 \cdot 9)\end{array}$ & $\begin{array}{l}65 \cdot 75 \\
(61 \cdot 8-69 \cdot 6)\end{array}$ &. & $\begin{array}{l}86 \cdot 65 \\
(78 \cdot 2-95 \cdot 0)\end{array}$ & .. \\
\hline \multicolumn{11}{|l|}{ Hong Kong* } \\
\hline 2000-04 &.. &. & .. & .. &.. & .. & .. &.. & .. & .. \\
\hline 2005-09 & $\begin{array}{l}82 \cdot 2 \\
(80 \cdot 9-83 \cdot 5)\end{array}$ & $\begin{array}{l}66 \cdot 7 \\
(64 \cdot 5-69 \cdot 0)\end{array}$ & .. &. &. & .. &. &. &. &. \\
\hline 2010-14 & $\begin{array}{l}83 \cdot 3 \\
(82 \cdot 1-84 \cdot 6)\end{array}$ & $\begin{array}{l}65 \cdot 8 \\
(63 \cdot 6-68 \cdot 1)\end{array}$ & .. &. & .. & .. & .. & .. &. & .. \\
\hline \multicolumn{11}{|c|}{ India (two registries) } \\
\hline 2000-04 & $\begin{array}{l}57 \cdot 6 \\
(48 \cdot 1-67 \cdot 1)\end{array}$ & $\begin{array}{l}45 \cdot 2 \\
(34 \cdot 8-55 \cdot 6)\end{array}$ & $\begin{array}{l}25 \cdot 6 \dagger \\
(12 \cdot 2-39 \cdot 1)\end{array}$ & $\begin{array}{l}24 \cdot 8 \dagger \\
(6 \cdot 3-43 \cdot 3)\end{array}$ & $\begin{array}{l}22 \cdot 4+5 \\
(8 \cdot 3-36 \cdot 5)\end{array}$ & $\begin{array}{l}19 \cdot 7 \dagger \\
(6 \cdot 3-33 \cdot 1)\end{array}$ & $\begin{array}{l}40 \cdot 6 \dagger \\
(28 \cdot 1-53 \cdot 1)\end{array}$ &. & $\begin{array}{l}54 \cdot 0 \dagger \\
(28 \cdot 3-79 \cdot 8)\end{array}$ & .. \\
\hline 2005-09 & $\begin{array}{l}59 \cdot 1 \\
(46 \cdot 6-71 \cdot 6)\end{array}$ & $\begin{array}{l}51 \cdot 6 \\
(40 \cdot 5-62 \cdot 6)\end{array}$ & $\begin{array}{l}13 \cdot 2 \\
(7 \cdot 7-18 \cdot 7)\end{array}$ & $\begin{array}{l}33 \cdot 2 \dagger \\
(16 \cdot 3-50 \cdot 0)\end{array}$ & $\begin{array}{l}16 \cdot 7 \\
(10 \cdot 4-23 \cdot 0)\end{array}$ & $\begin{array}{c}6 \cdot 1 \\
(2 \cdot 5-9 \cdot 6)\end{array}$ & $\begin{array}{l}45 \cdot 4 \\
(34 \cdot 2-56 \cdot 6)\end{array}$ & .. & $\begin{array}{l}75 \cdot 5 \dagger \\
(52 \cdot 1-99 \cdot 0)\end{array}$ & .. \\
\hline 2010-14 & $\begin{array}{l}66 \cdot 1 \\
(51 \cdot 5-80 \cdot 8)\end{array}$ & $\begin{array}{l}59 \cdot 0 \\
(47 \cdot 5-70 \cdot 5)\end{array}$ & $\begin{array}{l}15 \cdot 6 \\
(10 \cdot 2-21 \cdot 1)\end{array}$ & $\begin{array}{l}44 \cdot 3 \\
(32 \cdot 1-56 \cdot 6)\end{array}$ & $\begin{array}{l}30 \cdot 0 \dagger \\
(17 \cdot 5-42 \cdot 6)\end{array}$ & $\begin{array}{l}29 \cdot 0 \dagger \\
(16 \cdot 8-41 \cdot 3)\end{array}$ & $\begin{array}{l}45 \cdot 6 \\
(33 \cdot 0-58 \cdot 1)\end{array}$ & .. &. & .. \\
\hline \multicolumn{11}{|c|}{ Iran (Golestan) } \\
\hline 2000-04 &. & .. & .. &.$\cdot$ &.. & .. & .. & .. & .. & .. \\
\hline 2005-09 &.$\cdot$ & .. & .. &. & .. & .. & .. &.$\cdot$ & .. &. \\
\hline 2010-14 &.. &.. &. &. &.. & .. & .. &.. & .. & .. \\
\hline \multicolumn{11}{|l|}{ |srael* } \\
\hline 2000-04 & $\begin{array}{l}85 \cdot 1 \\
(84 \cdot 2-86 \cdot 1)\end{array}$ & $\begin{array}{l}65 \cdot 0 \\
(61 \cdot 6-68 \cdot 5)\end{array}$ & $\begin{array}{l}40 \cdot 1 \\
(37 \cdot 8-42 \cdot 4)\end{array}$ & $\begin{array}{l}92 \cdot 5 \\
(91 \cdot 4-93 \cdot 6)\end{array}$ & $\begin{array}{l}29 \cdot 7 \\
(27 \cdot 8-31 \cdot 7)\end{array}$ & $\begin{array}{l}51 \cdot 3 \\
(49 \cdot 1-53 \cdot 5)\end{array}$ & $\begin{array}{l}63 \cdot 7 \\
(62 \cdot 5-64 \cdot 9)\end{array}$ & $\begin{array}{l}74 \cdot 5 \\
(69 \cdot 3-79 \cdot 6)\end{array}$ & $\begin{array}{l}86 \cdot 4 \\
(81 \cdot 6-91 \cdot 2)\end{array}$ & $\begin{array}{c}89 \cdot 1 \\
(84 \cdot 6-93 \cdot 5)\end{array}$ \\
\hline 2005-09 & $\begin{array}{l}87 \cdot 8 \\
(86 \cdot 8-88 \cdot 7)\end{array}$ & $\begin{array}{l}65 \cdot 9 \\
(62 \cdot 7-69 \cdot 1)\end{array}$ & $\begin{array}{l}43 \cdot 5 \\
(41 \cdot 1-45 \cdot 9)\end{array}$ & $\begin{array}{l}95 \cdot 7 \\
(94 \cdot 7-96 \cdot 6)\end{array}$ & $\begin{array}{l}32 \cdot 3 \\
(30 \cdot 4-34 \cdot 3)\end{array}$ & $\begin{array}{l}43 \cdot 9 \\
(41 \cdot 9-46 \cdot 0)\end{array}$ & $\begin{array}{l}65 \cdot 2 \\
(64 \cdot 1-66 \cdot 3)\end{array}$ & $\begin{array}{l}72 \cdot 0 \\
(67 \cdot 2-76 \cdot 9)\end{array}$ & $\begin{array}{l}86 \cdot 7 \\
(82 \cdot 2-91 \cdot 2)\end{array}$ & $\begin{array}{c}90 \cdot 8 \\
(87 \cdot 3-94 \cdot 3)\end{array}$ \\
\hline 2010-14 & $\begin{array}{l}88 \cdot 0 \\
(87 \cdot 0-89 \cdot 0)\end{array}$ & $\begin{array}{l}66 \cdot 6 \\
(63 \cdot 2-70 \cdot 1)\end{array}$ & $\begin{array}{l}45 \cdot 0 \\
(42 \cdot 3-47 \cdot 7)\end{array}$ & $\begin{array}{l}95 \cdot 6 \\
(94 \cdot 5-96 \cdot 7)\end{array}$ & $\begin{array}{l}32 \cdot 8 \\
(30 \cdot 6-34 \cdot 9)\end{array}$ & $\begin{array}{l}39 \cdot 7 \\
(37 \cdot 6-41 \cdot 9)\end{array}$ & $\begin{array}{l}65 \cdot 5 \\
(64 \cdot 2-66 \cdot 7)\end{array}$ & $\begin{array}{l}77 \cdot 6 \\
(72 \cdot 6-82 \cdot 6)\end{array}$ & $\begin{array}{l}87 \cdot 9 \\
(83 \cdot 1-92 \cdot 6)\end{array}$ & $\begin{array}{c}92 \cdot 3 \\
(89 \cdot 0-95 \cdot 6)\end{array}$ \\
\hline \multicolumn{11}{|c|}{ Japan (16 registries) } \\
\hline 2000-04 & $\begin{array}{l}85 \cdot 9 \\
(85 \cdot 2-86 \cdot 6)\end{array}$ & $\begin{array}{l}67 \cdot 5 \\
(66 \cdot 3-68 \cdot 7)\end{array}$ & $\begin{array}{l}35 \cdot 5 \\
(33 \cdot 8-37 \cdot 2)\end{array}$ & $\begin{array}{l}85.9 \\
(84.9-87.0)\end{array}$ & $\begin{array}{l}27 \cdot 9 \Omega \\
(26 \cdot 3-29 \cdot 5)\end{array}$ & $\begin{array}{l}24 \cdot 8 \\
(23 \cdot 4-26 \cdot 1)\end{array}$ & $\begin{array}{l}47 \cdot 5 \\
(46 \cdot 3-48 \cdot 7)\end{array}$ & $\begin{array}{l}65 \cdot 3 \\
(59 \cdot 2-71 \cdot 5)\end{array}$ & $\begin{array}{l}79 \cdot 7 \\
(74 \cdot 9-84 \cdot 4)\end{array}$ & $\begin{array}{c}86 \cdot 0 \\
(80 \cdot 2-91 \cdot 7)\end{array}$ \\
\hline 2005-09 & $\begin{array}{l}88.9 \\
(88.4-89 \cdot 3)\end{array}$ & $\begin{array}{l}69 \cdot 2 \\
(68 \cdot 3-70 \cdot 1)\end{array}$ & $\begin{array}{l}43 \cdot 9 \\
(42 \cdot 8-45 \cdot 1)\end{array}$ & $\begin{array}{l}91 \cdot 4 \\
(90 \cdot 8-92 \cdot 0)\end{array}$ & $\begin{array}{l}38 \cdot 58 \\
(37 \cdot 2-39 \cdot 7)\end{array}$ & $\begin{array}{l}27 \cdot 5 \\
(26 \cdot 7-28 \cdot 3)\end{array}$ & $\begin{array}{l}52 \cdot 0 \\
(51 \cdot 4-52 \cdot 6)\end{array}$ & $\begin{array}{l}62.5 \\
(58.1-66.8)\end{array}$ & $\begin{array}{l}83 \cdot 7 \\
(80 \cdot 6-86 \cdot 9)\end{array}$ & $\begin{array}{c}84.7 \\
(79.5-89.9)\end{array}$ \\
\hline 2010-14 & $\begin{array}{l}89.4 \\
(88.9-89.9)\end{array}$ & $\begin{array}{l}71 \cdot 4 \\
(70 \cdot 4-72 \cdot 3)\end{array}$ & $\begin{array}{l}46 \cdot 3 \\
(44 \cdot 9-47 \cdot 7)\end{array}$ & $\begin{array}{l}93 \cdot 0 \\
(92 \cdot 4-93 \cdot 6)\end{array}$ & $\begin{array}{l}46 \cdot 38 \\
(44 \cdot 9-47 \cdot 7)\end{array}$ & $\begin{array}{l}33 \cdot 3 \\
(32 \cdot 4-34 \cdot 3)\end{array}$ & $\begin{array}{l}57 \cdot 3 \\
(56 \cdot 5-58 \cdot 0)\end{array}$ & $\begin{array}{l}69 \cdot 6 \\
(64 \cdot 4-74 \cdot 7)\end{array}$ & $\begin{array}{l}87 \cdot 6 \\
(84 \cdot 2-91 \cdot 0)\end{array}$ & $\begin{array}{c}89.6 \\
(84 \cdot 2-95 \cdot 0)\end{array}$ \\
\hline \multicolumn{11}{|l|}{ Jordan* } \\
\hline 2000-04 & $\begin{array}{l}87 \cdot 6 \$ \\
(83 \cdot 5-91 \cdot 7)\end{array}$ & $\begin{array}{l}75 \cdot 65 \\
(66 \cdot 8-84 \cdot 3)\end{array}$ & .. & $\begin{array}{l}88 \cdot 5 \S \\
(83.7-93 \cdot 3)\end{array}$ & $\begin{array}{l}55 \cdot 85 \\
(49 \cdot 0-62 \cdot 6)\end{array}$ & $\begin{array}{l}57 \cdot 15 \\
(48 \cdot 1-66 \cdot 1)\end{array}$ & $\begin{array}{l}76.05 \\
(69 \cdot 4-82 \cdot 6)\end{array}$ & $\begin{array}{l}73 \cdot 55 \\
(65 \cdot 7-81 \cdot 3)\end{array}$ & $\begin{array}{l}75 \cdot 4 \S \\
(69 \cdot 7-81 \cdot 2)\end{array}$ & $\begin{array}{c}92.4 \Omega \\
(88.1-96.6)\end{array}$ \\
\hline 2005-09 & $\begin{array}{l}86 \cdot 65 \\
(83 \cdot 2-90 \cdot 0)\end{array}$ & $\begin{array}{l}70 \cdot 65 \\
(63 \cdot 9-77 \cdot 4)\end{array}$ &.. & $\begin{array}{l}88.65 \\
(83 \cdot 3-93 \cdot 9)\end{array}$ & $\begin{array}{l}46 \cdot 78 \\
(41 \cdot 1-52 \cdot 4)\end{array}$ & $\begin{array}{l}56 \cdot 5 \S \\
(46 \cdot 9-66 \cdot 1)\end{array}$ & $\begin{array}{l}74 \cdot 65 \\
(68 \cdot 3-80 \cdot 9)\end{array}$ & $\begin{array}{l}66 \cdot 05 \\
(58 \cdot 6-73 \cdot 3)\end{array}$ & $\begin{array}{l}89 \cdot 25 \\
(84 \cdot 8-93 \cdot 6)\end{array}$ & $\begin{array}{c}91 \cdot 15 \\
(86 \cdot 4-95 \cdot 8)\end{array}$ \\
\hline 2010-14 & $\begin{array}{l}84.4 \Omega \\
(80.9-88.0)\end{array}$ & $\begin{array}{l}56 \cdot 4 \S \\
(48 \cdot 2-64 \cdot 6)\end{array}$ &.. & $\begin{array}{l}86 \cdot 1 \S \\
(81 \cdot 1-91 \cdot 0)\end{array}$ & $\begin{array}{l}32 \cdot 9 \$ \\
(26 \cdot 8-39 \cdot 0)\end{array}$ & $\begin{array}{l}42 \cdot 5 \S \\
(35 \cdot 3-49 \cdot 6)\end{array}$ & $\begin{array}{l}65 \cdot 15 \\
(60 \cdot 3-69 \cdot 9)\end{array}$ & $\begin{array}{l}57 \cdot 35 \\
(49 \cdot 9-64 \cdot 8)\end{array}$ & $\begin{array}{l}88.05 \\
(83.1-92.8)\end{array}$ & $\begin{array}{c}87.05 \\
(81.4-92.6)\end{array}$ \\
\hline \multicolumn{11}{|l|}{ Korea* } \\
\hline 2000-04 & $\begin{array}{l}79 \cdot 5 \\
(78 \cdot 0-81 \cdot 0)\end{array}$ & $\begin{array}{l}76 \cdot 0 \\
(75 \cdot 3-76 \cdot 7)\end{array}$ & $\begin{array}{l}43 \cdot 0 \\
(41 \cdot 2-44 \cdot 7)\end{array}$ & $\begin{array}{l}76 \cdot 0 \\
(74 \cdot 6-77 \cdot 5)\end{array}$ & $\begin{array}{l}27 \cdot 6 \\
(26 \cdot 4-28 \cdot 9)\end{array}$ & $\begin{array}{l}31 \cdot 7 \\
(30 \cdot 4-33 \cdot 0)\end{array}$ & $\begin{array}{l}39 \cdot 8 \\
(38 \cdot 8-40 \cdot 8)\end{array}$ & $\begin{array}{l}54 \cdot 2 \\
(50 \cdot 8-57 \cdot 6)\end{array}$ & $\begin{array}{l}73 \cdot 1 \\
(70 \cdot 4-75 \cdot 8)\end{array}$ & $\begin{array}{c}82 \cdot 4 \\
(79 \cdot 3-85 \cdot 4)\end{array}$ \\
\hline 2005-09 & $\begin{array}{l}84 \cdot 0 \\
(83 \cdot 0-85 \cdot 0)\end{array}$ & $\begin{array}{l}77 \cdot 0 \\
(76 \cdot 4-77 \cdot 7)\end{array}$ & $\begin{array}{l}44 \cdot 1 \\
(42 \cdot 7-45 \cdot 5)\end{array}$ & $\begin{array}{l}87 \cdot 3 \\
(86 \cdot 5-88 \cdot 1)\end{array}$ & $\begin{array}{l}31 \cdot 9 \\
(30 \cdot 8-33 \cdot 0)\end{array}$ & $\begin{array}{l}41 \cdot 5 \\
(40 \cdot 5-42 \cdot 5)\end{array}$ & $\begin{array}{l}47 \cdot 5 \\
(46 \cdot 7-48 \cdot 4)\end{array}$ & $\begin{array}{l}61 \cdot 4 \\
(58 \cdot 1-64 \cdot 7)\end{array}$ & $\begin{array}{l}78 \cdot 6 \\
(76 \cdot 1-81 \cdot 0)\end{array}$ & $\begin{array}{c}83.0 \\
(80 \cdot 0-85.9)\end{array}$ \\
\hline \multirow[t]{2}{*}{ 2010-14 } & $\begin{array}{l}86 \cdot 6 \\
(85 \cdot 8-87 \cdot 5)\end{array}$ & $\begin{array}{l}77 \cdot 3 \\
(76 \cdot 6-78 \cdot 0)\end{array}$ & $\begin{array}{l}47 \cdot 5 \\
(46 \cdot 2-48 \cdot 9)\end{array}$ & $\begin{array}{l}89.9 \\
(89 \cdot 2-90 \cdot 5)\end{array}$ & $\begin{array}{l}33 \cdot 7 \\
(32 \cdot 6-34 \cdot 7)\end{array}$ & $\begin{array}{l}45 \cdot 9 \\
(44 \cdot 9-46 \cdot 8)\end{array}$ & $\begin{array}{l}52 \cdot 5 \\
(51 \cdot 8-53 \cdot 3)\end{array}$ & $\begin{array}{l}60 \cdot 3 \\
(56 \cdot 9-63 \cdot 8)\end{array}$ & $\begin{array}{l}84 \cdot 4 \\
(82 \cdot 1-86 \cdot 7)\end{array}$ & $\begin{array}{c}91.0 \\
(88.9-93 \cdot 1)\end{array}$ \\
\hline & & & & & & & & & \multicolumn{2}{|c|}{ (Table 7 continues on next page } \\
\hline
\end{tabular}




\begin{tabular}{|c|c|c|c|c|c|c|c|c|c|c|}
\hline & \multicolumn{3}{|c|}{ Women's cancers } & \multirow[t]{2}{*}{ Prostate } & \multirow[t]{2}{*}{$\begin{array}{l}\text { Brain } \\
\text { (adults) }\end{array}$} & \multicolumn{2}{|c|}{$\begin{array}{l}\text { Haemopoietic malignancies } \\
\text { (adults) }\end{array}$} & \multicolumn{3}{|c|}{ Childhood malignancies } \\
\hline & Breast & Cervix & Ovary & & & Myeloid & Lymphoid & Brain & $\begin{array}{l}\text { Acute } \\
\text { lymphoblastic } \\
\text { leukaemia }\end{array}$ & Lymphoma \\
\hline \multicolumn{11}{|c|}{ (Continued from previous page) } \\
\hline \multicolumn{11}{|l|}{ Kuwait* } \\
\hline 2000-04 & $\begin{array}{l}68 \cdot 3 \\
(58 \cdot 0-78 \cdot 7)\end{array}$ & $\begin{array}{l}54 \cdot 8 \\
(45 \cdot 2-64 \cdot 3)\end{array}$ & $\begin{array}{l}38 \cdot 9 \dagger \\
(26 \cdot 3-51 \cdot 5)\end{array}$ & $\begin{array}{l}78.8 \dagger \\
(66.7-90.9)\end{array}$ & $\begin{array}{l}30 \cdot 3+5 \\
(19 \cdot 1-41 \cdot 5)\end{array}$ & $\begin{array}{l}38 \cdot 6 \\
(27 \cdot 1-50 \cdot 0)\end{array}$ & $\begin{array}{l}52 \cdot 1 \\
(42 \cdot 9-61 \cdot 2)\end{array}$ & $\begin{array}{l}47 \cdot 1 \dagger \\
(24 \cdot 4-69 \cdot 8)\end{array}$ & $\begin{array}{l}76 \cdot 15 \\
(65 \cdot 7-86 \cdot 5)\end{array}$ & $\begin{array}{c}93 \cdot 0 \\
(86 \cdot 2-99 \cdot 8)\end{array}$ \\
\hline 2005-09 & $\begin{array}{l}71 \cdot 0 \\
(63 \cdot 8-78 \cdot 2)\end{array}$ & $\begin{array}{l}73 \cdot 8 \dagger \\
(61 \cdot 7-86 \cdot 0)\end{array}$ & $\begin{array}{l}35 \cdot 4 \\
(25 \cdot 2-45 \cdot 6)\end{array}$ & $\begin{array}{l}71 \cdot 9 \\
(63 \cdot 7-80 \cdot 0)\end{array}$ & $\begin{array}{l}24 \cdot 9 \\
(17 \cdot 3-32 \cdot 6)\end{array}$ & $\begin{array}{l}24 \cdot 0 \\
(15 \cdot 9-32 \cdot 0)\end{array}$ & $\begin{array}{l}63 \cdot 2 \\
(55 \cdot 8-70 \cdot 7)\end{array}$ & $\begin{array}{l}59 \cdot 2 \dagger \\
(39 \cdot 2-79 \cdot 1)\end{array}$ & $\begin{array}{l}74 \cdot 9 \\
(65 \cdot 6-84 \cdot 1)\end{array}$ & $\begin{array}{c}90 \cdot 7 \\
(83 \cdot 2-98 \cdot 2)\end{array}$ \\
\hline 2010-14 & $\begin{array}{l}75 \cdot 2 \\
(66 \cdot 4-83 \cdot 9)\end{array}$ & $\begin{array}{l}56 \cdot 6 \\
(44 \cdot 2-69 \cdot 0)\end{array}$ & $\begin{array}{l}35 \cdot 1 \\
(25 \cdot 6-44 \cdot 7)\end{array}$ & $\begin{array}{l}84 \cdot 0 \\
(74 \cdot 1-94 \cdot 0)\end{array}$ & $\begin{array}{l}31 \cdot 8 \\
(23 \cdot 2-40 \cdot 4)\end{array}$ & $\begin{array}{l}25 \cdot 6 \\
(17 \cdot 7-33 \cdot 6)\end{array}$ & $\begin{array}{l}68 \cdot 2 \\
(59 \cdot 5-76 \cdot 9)\end{array}$ &. & $\begin{array}{l}88 \cdot 4 \\
(80 \cdot 6-96 \cdot 2)\end{array}$ & $\begin{array}{c}96 \cdot 3 \\
(91 \cdot 4-100 \cdot 0)\end{array}$ \\
\hline \multicolumn{11}{|c|}{ Malaysia (Penang) } \\
\hline 2000-04 &.. & .. &.. & .. & .. &.$\cdot$ & .. & .. & .. & .. \\
\hline 2005-09 & $\begin{array}{l}74 \cdot 45 \\
(66 \cdot 3-82 \cdot 4)\end{array}$ & $\begin{array}{l}56 \cdot 35 \\
(49 \cdot 5-63 \cdot 0)\end{array}$ & $\begin{array}{l}36 \cdot 45 \\
(27 \cdot 3-45 \cdot 6)\end{array}$ & $\begin{array}{l}74 \cdot 9 \\
(67 \cdot 5-82 \cdot 3)\end{array}$ & $\begin{array}{l}20 \cdot 5 \dagger \\
(10 \cdot 7-30 \cdot 4)\end{array}$ & $\begin{array}{l}20 \cdot 3 \\
(11 \cdot 5-29 \cdot 1)\end{array}$ & $\begin{array}{l}31 \cdot 4 \\
(24 \cdot 7-38 \cdot 1)\end{array}$ & $\begin{array}{l}41 \cdot 3+5 \\
(19 \cdot 0-63 \cdot 6)\end{array}$ & $\begin{array}{l}72 \cdot 85 \\
(62 \cdot 4-83 \cdot 3)\end{array}$ & $\begin{array}{c}95 \cdot 35 \\
(89 \cdot 2-100 \cdot 0)\end{array}$ \\
\hline 2010-14 & $\begin{array}{l}65 \cdot 05 \\
(52 \cdot 1-78 \cdot 0)\end{array}$ & $\begin{array}{l}57 \cdot 15 \\
(48 \cdot 3-65 \cdot 9)\end{array}$ & $\begin{array}{l}46 \cdot 85 \\
(34 \cdot 5-59 \cdot 0)\end{array}$ & $\begin{array}{l}87 \cdot 7 \\
(80 \cdot 8-94 \cdot 5)\end{array}$ & $\begin{array}{l}28 \cdot 3 \dagger \\
(14 \cdot 7-42 \cdot 0)\end{array}$ & $\begin{array}{l}29 \cdot 6 \\
(21 \cdot 2-38 \cdot 0)\end{array}$ & $\begin{array}{l}51 \cdot 3 \\
(39 \cdot 6-63 \cdot 1)\end{array}$ & $\begin{array}{l}63 \cdot 4+5 \\
(45 \cdot 7-81 \cdot 2)\end{array}$ & $\begin{array}{l}82 \cdot 35 \\
(72 \cdot 0-92 \cdot 5)\end{array}$ & $\begin{array}{c}85 \cdot 0+5 \\
(66 \cdot 2-100 \cdot 0)\end{array}$ \\
\hline \multicolumn{11}{|l|}{ Mongolia* } \\
\hline $2000-04$ & .. & .. &.. & .. &.. &.$\cdot$ & .. & .. & .. & .. \\
\hline 2005-09 & $\begin{array}{l}76 \cdot 7 \\
(60 \cdot 0-93 \cdot 4)\end{array}$ & .. &.. &.$\cdot$ &.$\cdot$ &.$\cdot$ & .. & .. & .. & .. \\
\hline 2010-14 & $\begin{array}{l}76 \cdot 1 \\
(63 \cdot 8-88 \cdot 4)\end{array}$ &.. &.. &.$\cdot$ &.. &.$\cdot$ & .. & .. & .. &. \\
\hline \multicolumn{11}{|l|}{ Qatar* } \\
\hline $2000-04$ & $\begin{array}{l}59 \cdot 25 \\
(48 \cdot 7-69 \cdot 7)\end{array}$ & $\begin{array}{l}83 \cdot 9+5 \\
(68 \cdot 5-99 \cdot 2)\end{array}$ & $\begin{array}{l}47 \cdot 1+5 \\
(25 \cdot 1-69 \cdot 0)\end{array}$ & $\begin{array}{l}81 \cdot 5+5 \\
(60 \cdot 7-100 \cdot 0)\end{array}$ & $\begin{array}{l}52 \cdot 9+5 \\
(34 \cdot 0-71 \cdot 9)\end{array}$ & $\begin{array}{l}63 \cdot 8+5 \\
(49 \cdot 8-77 \cdot 7)\end{array}$ & $\begin{array}{l}46 \cdot 55 \\
(34 \cdot 5-58 \cdot 4)\end{array}$ & $\begin{array}{l}11 \cdot 1+5 \\
(0 \cdot 0-27 \cdot 1)\end{array}$ & $\begin{array}{l}67 \cdot 3+5 \\
(44 \cdot 3-90 \cdot 3)\end{array}$ & $\begin{array}{l}69.05 \\
(52 \cdot 6-85 \cdot 4)\end{array}$ \\
\hline 2005-09 & $\begin{array}{l}73 \cdot 35 \\
(63 \cdot 0-83 \cdot 7)\end{array}$ & $\begin{array}{l}55 \cdot 6+5 \\
(35 \cdot 3-76 \cdot 0)\end{array}$ & $\begin{array}{l}62 \cdot 6+5 \\
(47 \cdot 5-77 \cdot 6)\end{array}$ & $\begin{array}{l}98 \cdot 2+5 \\
(87 \cdot 1-100 \cdot 0)\end{array}$ & $\begin{array}{l}32 \cdot 4+5 \\
(19 \cdot 0-45 \cdot 8)\end{array}$ & $\begin{array}{l}52 \cdot 1+5 \\
(39 \cdot 7-64 \cdot 6)\end{array}$ & $\begin{array}{l}60 \cdot 35 \\
(45 \cdot 9-74 \cdot 7)\end{array}$ & .. & $\begin{array}{l}82 \cdot 6+5 \\
(61 \cdot 2-100 \cdot 0)\end{array}$ & $\begin{array}{c}88 \cdot 5+5 \\
(73 \cdot 8-100 \cdot 0)\end{array}$ \\
\hline 2010-14 & $\begin{array}{l}71 \cdot 9 \S \\
(58 \cdot 4-85 \cdot 5)\end{array}$ & $\begin{array}{l}63 \cdot 5+5 \\
(44 \cdot 2-82 \cdot 8)\end{array}$ & $\begin{array}{l}39 \cdot 25 \\
(26 \cdot 3-52 \cdot 1)\end{array}$ & $\begin{array}{l}89 \cdot 6 \$ \\
(79 \cdot 0-100 \cdot 0)\end{array}$ & $\begin{array}{l}36 \cdot 1+5 \\
(20 \cdot 5-51 \cdot 7)\end{array}$ & $\begin{array}{l}56 \cdot 9 \$ \\
(42 \cdot 0-71 \cdot 9)\end{array}$ & $\begin{array}{l}75 \cdot 0 \Omega \\
(58 \cdot 8-91 \cdot 2)\end{array}$ & $\begin{array}{l}65 \cdot 5+5 \\
(34 \cdot 5-96 \cdot 5)\end{array}$ & $\begin{array}{l}100 \cdot 0 \$ \\
(100 \cdot 0-100 \cdot 0)\end{array}$ & $\begin{array}{c}95 \cdot 35 \\
(87 \cdot 3-100 \cdot 0)\end{array}$ \\
\hline \multicolumn{11}{|l|}{ Singapore* } \\
\hline $2000-04$ & $\begin{array}{l}76 \cdot 3 \\
(73 \cdot 9-78 \cdot 7)\end{array}$ & $\begin{array}{l}68 \cdot 3 \\
(65 \cdot 2-71 \cdot 5)\end{array}$ & $\begin{array}{l}42 \cdot 5 \\
(37 \cdot 9-47 \cdot 0)\end{array}$ & $\begin{array}{l}83 \cdot 4 \\
(80 \cdot 3-86 \cdot 5)\end{array}$ & $\begin{array}{l}26 \cdot 2 \\
(21 \cdot 5-30 \cdot 9)\end{array}$ & $\begin{array}{l}39 \cdot 2 \\
(35 \cdot 1-43 \cdot 3)\end{array}$ & $\begin{array}{l}46 \cdot 0 \\
(43 \cdot 0-48 \cdot 9)\end{array}$ & $\begin{array}{l}60 \cdot 9 \\
(48 \cdot 0-73 \cdot 8)\end{array}$ & $\begin{array}{l}79 \cdot 8 \\
(72 \cdot 3-87 \cdot 2)\end{array}$ & $\begin{array}{c}86 \cdot 9 \\
(77 \cdot 5-96 \cdot 3)\end{array}$ \\
\hline 2005-09 & $\begin{array}{l}80 \cdot 3 \\
(78 \cdot 3-82 \cdot 3)\end{array}$ & $\begin{array}{l}63 \cdot 3 \\
(60 \cdot 1-66 \cdot 6)\end{array}$ & $\begin{array}{l}46 \cdot 8 \\
(42 \cdot 8-50 \cdot 7)\end{array}$ & $\begin{array}{l}86 \cdot 7 \\
(84 \cdot 5-88 \cdot 9)\end{array}$ & $\begin{array}{l}30 \cdot 3 \\
(26 \cdot 2-34 \cdot 4)\end{array}$ & $\begin{array}{l}43 \cdot 7 \\
(40 \cdot 1-47 \cdot 4)\end{array}$ & $\begin{array}{l}55 \cdot 3 \\
(52 \cdot 7-57 \cdot 9)\end{array}$ & $\begin{array}{l}57 \cdot 5 \\
(45 \cdot 9-69 \cdot 0)\end{array}$ & $\begin{array}{l}90 \cdot 4 \\
(84 \cdot 2-96 \cdot 6)\end{array}$ & $\begin{array}{c}73 \cdot 8 \\
(61 \cdot 6-86 \cdot 0)\end{array}$ \\
\hline 2010-14 & $\begin{array}{l}80 \cdot 3 \\
(78 \cdot 4-82 \cdot 2)\end{array}$ & $\begin{array}{l}63 \cdot 4 \\
(60 \cdot 2-66 \cdot 6)\end{array}$ & $\begin{array}{l}43 \cdot 9 \\
(40 \cdot 7-47 \cdot 0)\end{array}$ & $\begin{array}{l}87.8 \\
(85 \cdot 8-89 \cdot 8)\end{array}$ & $\begin{array}{l}34 \cdot 4 \\
(30 \cdot 6-38 \cdot 2)\end{array}$ & $\begin{array}{l}44 \cdot 1 \\
(41 \cdot 1-47 \cdot 1)\end{array}$ & $\begin{array}{l}58 \cdot 7 \\
(56 \cdot 4-61 \cdot 1)\end{array}$ & $\begin{array}{l}62 \cdot 0 \\
(51 \cdot 4-72 \cdot 5)\end{array}$ & $\begin{array}{l}88 \cdot 6 \\
(81 \cdot 8-95 \cdot 4)\end{array}$ & $\begin{array}{c}92 \cdot 1 \\
(85 \cdot 2-99 \cdot 1)\end{array}$ \\
\hline \multicolumn{11}{|l|}{ Taiwan* } \\
\hline $2000-04$ & $\begin{array}{l}80 \cdot 2 \\
(78 \cdot 9-81 \cdot 6)\end{array}$ & $\begin{array}{l}74 \cdot 3 \\
(73 \cdot 4-75 \cdot 2)\end{array}$ & $\begin{array}{l}44 \cdot 1 \\
(41 \cdot 8-46 \cdot 4)\end{array}$ & $\begin{array}{l}75 \cdot 5 \\
(73 \cdot 8-77 \cdot 2)\end{array}$ & $\begin{array}{l}26 \cdot 5 \\
(24 \cdot 8-28 \cdot 1)\end{array}$ & $\begin{array}{l}23 \cdot 1 \\
(21 \cdot 6-24 \cdot 6)\end{array}$ & $\begin{array}{l}40 \cdot 3 \\
(39 \cdot 2-41 \cdot 4)\end{array}$ & $\begin{array}{l}57 \cdot 1 \\
(52 \cdot 4-61 \cdot 8)\end{array}$ & $\begin{array}{l}72 \cdot 1 \\
(68 \cdot 2-75 \cdot 9)\end{array}$ & $\begin{array}{c}80 \cdot 1 \\
(75 \cdot 6-84 \cdot 7)\end{array}$ \\
\hline $2005-09$ & $\begin{array}{l}82 \cdot 2 \\
(81 \cdot 2-83 \cdot 2)\end{array}$ & $\begin{array}{l}73 \cdot 2 \\
(72 \cdot 2-74 \cdot 2)\end{array}$ & $\begin{array}{l}47 \cdot 5 \\
(45 \cdot 5-49 \cdot 5)\end{array}$ & $\begin{array}{l}79 \cdot 8 \\
(78 \cdot 7-81 \cdot 0)\end{array}$ & $\begin{array}{l}27 \cdot 6 \\
(26 \cdot 0-29 \cdot 2)\end{array}$ & $\begin{array}{l}30 \cdot 4 \\
(29 \cdot 1-31 \cdot 7)\end{array}$ & $\begin{array}{l}46 \cdot 8 \\
(45 \cdot 8-47 \cdot 8)\end{array}$ & $\begin{array}{l}58 \cdot 8 \\
(54 \cdot 1-63 \cdot 5)\end{array}$ & $\begin{array}{l}78 \cdot 8 \\
(75 \cdot 3-82 \cdot 3)\end{array}$ & $\begin{array}{l}84 \cdot 0 \\
(79 \cdot 6-88 \cdot 4)\end{array}$ \\
\hline $2010-14$ & $\begin{array}{l}84 \cdot 2 \\
(83 \cdot 3-85 \cdot 1)\end{array}$ & $\begin{array}{l}70 \cdot 0 \\
(68 \cdot 9-71 \cdot 1)\end{array}$ & $\begin{array}{l}48 \cdot 8 \\
(46 \cdot 9-50 \cdot 8)\end{array}$ & $\begin{array}{l}83.0 \\
(81 \cdot 9-84 \cdot 0)\end{array}$ & $\begin{array}{l}28 \cdot 4 \\
(26 \cdot 9-29 \cdot 9)\end{array}$ & $\begin{array}{l}33 \cdot 4 \\
(32 \cdot 2-34 \cdot 6)\end{array}$ & $\begin{array}{l}50 \cdot 5 \\
(49 \cdot 6-51 \cdot 5)\end{array}$ & $\begin{array}{l}54 \cdot 8 \\
(49 \cdot 9-59 \cdot 6)\end{array}$ & $\begin{array}{l}76 \cdot 5 \\
(72 \cdot 8-80 \cdot 1)\end{array}$ & $\begin{array}{c}86 \cdot 7 \\
(82 \cdot 5-90 \cdot 8)\end{array}$ \\
\hline \multicolumn{11}{|c|}{ Thailand (six registries) } \\
\hline $2000-04$ & $\begin{array}{l}56 \cdot 65 \\
(52 \cdot 0-61 \cdot 3)\end{array}$ & $\begin{array}{l}53 \cdot 5 \S \\
(51 \cdot 3-55 \cdot 7)\end{array}$ & $\begin{array}{l}41 \cdot 85 \\
(34 \cdot 8-48 \cdot 7)\end{array}$ & $\begin{array}{l}52 \cdot 15 \\
(46 \cdot 6-57 \cdot 6)\end{array}$ & $\begin{array}{l}11 \cdot 75 \\
(9 \cdot 2-14 \cdot 3)\end{array}$ & $\begin{array}{c}9 \cdot 45 \\
(7 \cdot 2-11 \cdot 6)\end{array}$ & $\begin{array}{l}30 \cdot 35 \\
(26 \cdot 7-34 \cdot 0)\end{array}$ & $\begin{array}{l}23 \cdot 45 \\
(15 \cdot 2-31 \cdot 5)\end{array}$ & $\begin{array}{l}54 \cdot 5 \$ \\
(46 \cdot 7-62 \cdot 4)\end{array}$ & $\begin{array}{c}43 \cdot 9+5 \\
(27 \cdot 1-60 \cdot 8)\end{array}$ \\
\hline 2005-09 & $\begin{array}{l}64.85 \\
(62.5-67 \cdot 0)\end{array}$ & $\begin{array}{l}55 \cdot 85 \\
(54 \cdot 0-57 \cdot 7)\end{array}$ & $\begin{array}{l}35 \cdot 8 \\
(32 \cdot 3-39 \cdot 3)\end{array}$ & $\begin{array}{l}71 \cdot 8 \\
(69 \cdot 0-74 \cdot 7)\end{array}$ & $\begin{array}{l}17 \cdot 6 \\
(15 \cdot 2-20 \cdot 0)\end{array}$ & $\begin{array}{l}18 \cdot 2 \\
(16 \cdot 0-20 \cdot 4)\end{array}$ & $\begin{array}{l}36 \cdot 1 \\
(33 \cdot 7-38 \cdot 4)\end{array}$ & $\begin{array}{l}38 \cdot 4 \\
(30 \cdot 6-46 \cdot 3)\end{array}$ & $\begin{array}{l}60 \cdot 6 \\
(54 \cdot 2-67 \cdot 1)\end{array}$ & $\begin{array}{c}63 \cdot 75 \\
(52 \cdot 3-75 \cdot 0)\end{array}$ \\
\hline 2010-14 & $\begin{array}{l}68 \cdot 7 \S \\
(66 \cdot 6-70 \cdot 8)\end{array}$ & $\begin{array}{l}53 \cdot 99 \\
(52 \cdot 1-55 \cdot 8)\end{array}$ & $\begin{array}{l}37 \cdot 2 \\
(34 \cdot 0-40 \cdot 5)\end{array}$ & $\begin{array}{l}68 \cdot 0 \\
(65 \cdot 2-70 \cdot 8)\end{array}$ & $\begin{array}{l}14 \cdot 7 \\
(12 \cdot 6-16 \cdot 9)\end{array}$ & $\begin{array}{l}25 \cdot 4 \\
(22 \cdot 8-28 \cdot 0)\end{array}$ & $\begin{array}{l}35 \cdot 0 \\
(32 \cdot 8-37 \cdot 1)\end{array}$ & $\begin{array}{l}44 \cdot 5 \$ \\
(35 \cdot 9-53 \cdot 1)\end{array}$ & $\begin{array}{l}65 \cdot 95 \\
(59 \cdot 2-72 \cdot 6)\end{array}$ & $\begin{array}{c}73.95 \\
(65 \cdot 0-82 \cdot 7)\end{array}$ \\
\hline
\end{tabular}




\begin{tabular}{|c|c|c|c|c|c|c|c|c|c|c|}
\hline & \multicolumn{3}{|c|}{ Women's cancers } & \multirow[t]{2}{*}{ Prostate } & \multirow[t]{2}{*}{$\begin{array}{l}\text { Brain } \\
\text { (adults) }\end{array}$} & \multicolumn{2}{|c|}{$\begin{array}{l}\text { Haemopoietic malignancies } \\
\text { (adults) }\end{array}$} & \multicolumn{3}{|c|}{ Childhood malignancies } \\
\hline & Breast & Cervix & Ovary & & & Myeloid & Lymphoid & Brain & $\begin{array}{l}\text { Acute } \\
\text { lymphoblastic } \\
\text { leukaemia }\end{array}$ & Lymphoma \\
\hline \multicolumn{11}{|c|}{ (Continued from previous page) } \\
\hline \multicolumn{11}{|c|}{ Turkey (nine registries) } \\
\hline $2000-04$ & $\begin{array}{l}84 \cdot 8 \\
(81 \cdot 5-88 \cdot 0)\end{array}$ & $\begin{array}{l}64 \cdot 8 \\
(59 \cdot 7-69 \cdot 9)\end{array}$ & $\begin{array}{l}46 \cdot 15 \\
(38 \cdot 3-53 \cdot 8)\end{array}$ & $\begin{array}{l}83 \cdot 45 \\
(79 \cdot 3-87 \cdot 5)\end{array}$ & $\begin{array}{l}32 \cdot 15 \\
(27 \cdot 9-36 \cdot 2)\end{array}$ & $\begin{array}{l}35 \cdot 9 \$ \\
(30 \cdot 6-41 \cdot 2)\end{array}$ & $\begin{array}{l}54 \cdot 35 \\
(49 \cdot 6-58 \cdot 9)\end{array}$ & $\begin{array}{l}56 \cdot 8 \\
(46 \cdot 3-67 \cdot 2)\end{array}$ & $\begin{array}{l}72 \cdot 4 \\
(64 \cdot 3-80 \cdot 6)\end{array}$ & $\begin{array}{c}79 \cdot 9 \\
(70 \cdot 9-88 \cdot 9)\end{array}$ \\
\hline 2005-09 & $\begin{array}{l}80 \cdot 6 \\
(79 \cdot 2-82 \cdot 1)\end{array}$ & $\begin{array}{l}59 \cdot 2 \\
(56 \cdot 5-61 \cdot 9)\end{array}$ & $\begin{array}{l}40 \cdot 0 \\
(37 \cdot 4-42 \cdot 6)\end{array}$ & $\begin{array}{l}81 \cdot 2 \\
(79 \cdot 9-82 \cdot 6)\end{array}$ & $\begin{array}{l}34 \cdot 0 \\
(32 \cdot 4-35 \cdot 6)\end{array}$ & $\begin{array}{l}49 \cdot 0 \\
(46 \cdot 8-51 \cdot 2)\end{array}$ & $\begin{array}{l}50 \cdot 3 \\
(48 \cdot 7-51 \cdot 8)\end{array}$ & $\begin{array}{l}61 \cdot 1 \\
(56 \cdot 2-66 \cdot 0)\end{array}$ & $\begin{array}{l}78 \cdot 7 \\
(74 \cdot 9-82 \cdot 6)\end{array}$ & $\begin{array}{c}85 \cdot 0 \\
(81 \cdot 4-88 \cdot 7)\end{array}$ \\
\hline 2010-14 & $\begin{array}{l}82 \cdot 1 \\
(80 \cdot 7-83 \cdot 5)\end{array}$ & $\begin{array}{l}60 \cdot 7 \\
(58 \cdot 1-63 \cdot 3)\end{array}$ & $\begin{array}{l}39 \cdot 7 \\
(37 \cdot 3-42 \cdot 0)\end{array}$ & $\begin{array}{l}83 \cdot 8 \\
(82 \cdot 5-85 \cdot 1)\end{array}$ & $\begin{array}{l}35 \cdot 6 \\
(34 \cdot 1-37 \cdot 0)\end{array}$ & $\begin{array}{l}54 \cdot 0 \\
(51 \cdot 8-56 \cdot 1)\end{array}$ & $\begin{array}{l}54 \cdot 5 \\
(53 \cdot 0-56 \cdot 1)\end{array}$ & $\begin{array}{l}62 \cdot 5 \\
(58 \cdot 4-66 \cdot 7)\end{array}$ & $\begin{array}{l}80 \cdot 9 \\
(77 \cdot 2-84 \cdot 6)\end{array}$ & $\begin{array}{c}82 \cdot 9 \\
(79 \cdot 1-86 \cdot 7)\end{array}$ \\
\hline \multicolumn{11}{|l|}{ Europe } \\
\hline \multicolumn{11}{|l|}{ Austria* } \\
\hline $2000-04$ & $\begin{array}{l}81 \cdot 7 \\
(80 \cdot 9-82 \cdot 4)\end{array}$ & $\begin{array}{l}65 \cdot 4 \\
(63 \cdot 4-67 \cdot 4)\end{array}$ & $\begin{array}{l}40 \cdot 9 \\
(39 \cdot 4-42 \cdot 5)\end{array}$ & $\begin{array}{l}90 \cdot 1 \\
(89 \cdot 3-90 \cdot 8)\end{array}$ & $\begin{array}{l}24 \cdot 2 \\
(22 \cdot 6-25 \cdot 9)\end{array}$ & $\begin{array}{l}25 \cdot 0 \\
(23 \cdot 1-26 \cdot 8)\end{array}$ & $\begin{array}{l}57 \cdot 6 \\
(56 \cdot 5-58 \cdot 7)\end{array}$ &.. &. &.. \\
\hline 2005-09 & $\begin{array}{l}83 \cdot 9 \\
(83 \cdot 2-84 \cdot 6)\end{array}$ & $\begin{array}{l}66 \cdot 0 \\
(63 \cdot 7-68 \cdot 2)\end{array}$ & $\begin{array}{l}41 \cdot 2 \\
(39 \cdot 6-42 \cdot 8)\end{array}$ & $\begin{array}{l}90 \cdot 8 \\
(90 \cdot 1-91 \cdot 5)\end{array}$ & $\begin{array}{l}28 \cdot 5 \\
(26 \cdot 9-30 \cdot 1)\end{array}$ & $\begin{array}{l}29 \cdot 6 \\
(27 \cdot 7-31 \cdot 5)\end{array}$ & $\begin{array}{l}62 \cdot 2 \\
(61 \cdot 1-63 \cdot 3)\end{array}$ &.. &.. &.. \\
\hline 2010-14 & $\begin{array}{l}84 \cdot 8 \\
(84 \cdot 1-85 \cdot 5)\end{array}$ & $\begin{array}{l}63 \cdot 9 \\
(61 \cdot 6-66 \cdot 2)\end{array}$ & $\begin{array}{l}41 \cdot 0 \\
(39 \cdot 4-42 \cdot 7)\end{array}$ & $\begin{array}{l}90 \cdot 2 \\
(89 \cdot 5-90 \cdot 9)\end{array}$ & $\begin{array}{l}26 \cdot 3 \\
(24 \cdot 7-27 \cdot 9)\end{array}$ & $\begin{array}{l}32 \cdot 0 \\
(30 \cdot 1-34 \cdot 0)\end{array}$ & $\begin{array}{l}63 \cdot 3 \\
(62 \cdot 3-64 \cdot 4)\end{array}$ &. &. &. \\
\hline \multicolumn{11}{|c|}{ Belarus (childhood) $\ddagger$} \\
\hline 2000-04 &.. &.. &.. &.. &. &. &. & $\begin{array}{l}66 \cdot 1 \\
(59 \cdot 8-72 \cdot 3)\end{array}$ & $\begin{array}{l}78 \cdot 5 \\
(73 \cdot 0-83 \cdot 9)\end{array}$ & $\begin{array}{c}86 \cdot 5 \\
(80 \cdot 7-92 \cdot 3)\end{array}$ \\
\hline 2005-09 & $\cdot$. &.$\cdot$ & .. & $\cdot$. &. & $\cdot$. & $\cdot$. & $\begin{array}{l}70 \cdot 4 \\
(64 \cdot 0-76 \cdot 8)\end{array}$ & $\begin{array}{l}87 \cdot 0 \\
(81 \cdot 9-92 \cdot 2)\end{array}$ & $\begin{array}{c}84 \cdot 6 \\
(77 \cdot 0-92 \cdot 2)\end{array}$ \\
\hline 2010-14 &.. & $\cdot$. &.. & $\cdot$. &. & $\cdot$. & .. & $\begin{array}{l}68 \cdot 5 \\
(61 \cdot 6-75 \cdot 5)\end{array}$ & $\begin{array}{l}86 \cdot 6 \\
(81 \cdot 7-91 \cdot 5)\end{array}$ & $\begin{array}{c}85 \cdot 2 \\
(78 \cdot 0-92 \cdot 4)\end{array}$ \\
\hline \multicolumn{11}{|l|}{ Belgium* } \\
\hline $2000-04$ & $\begin{array}{l}84 \cdot 8 \\
(83 \cdot 5-86 \cdot 0)\end{array}$ & $\begin{array}{l}65 \cdot 1 \\
(61 \cdot 1-69 \cdot 0)\end{array}$ & $\begin{array}{l}42 \cdot 7 \\
(39 \cdot 4-45 \cdot 9)\end{array}$ & $\begin{array}{l}92 \cdot 1 \\
(90 \cdot 8-93 \cdot 4)\end{array}$ & $\begin{array}{l}31 \cdot 5 \\
(28 \cdot 5-34 \cdot 5)\end{array}$ & $\begin{array}{l}49 \cdot 7 \\
(46 \cdot 7-52 \cdot 7)\end{array}$ & $\begin{array}{l}65 \cdot 5 \\
(63 \cdot 6-67 \cdot 4)\end{array}$ & $\begin{array}{l}84 \cdot 1 \\
(75 \cdot 6-92 \cdot 6)\end{array}$ & $\begin{array}{l}80 \cdot 4 \\
(69 \cdot 8-91 \cdot 0)\end{array}$ & $\begin{array}{c}94 \cdot 2 \\
(87 \cdot 9-100 \cdot 0)\end{array}$ \\
\hline 2005-09 & $\begin{array}{l}85 \cdot 3 \\
(84 \cdot 7-85 \cdot 8)\end{array}$ & $\begin{array}{l}65 \cdot 7 \\
(63 \cdot 8-67 \cdot 6)\end{array}$ & $\begin{array}{l}42 \cdot 8 \\
(41 \cdot 3-44 \cdot 3)\end{array}$ & $\begin{array}{l}93 \cdot 2 \\
(92 \cdot 6-93 \cdot 7)\end{array}$ & $\begin{array}{l}31 \cdot 9 \\
(30 \cdot 6-33 \cdot 2)\end{array}$ & $\begin{array}{l}53 \cdot 2 \\
(51 \cdot 9-54 \cdot 5)\end{array}$ & $\begin{array}{l}68 \cdot 0 \\
(67 \cdot 2-68 \cdot 8)\end{array}$ & $\begin{array}{l}75 \cdot 2 \\
(70 \cdot 3-80 \cdot 1)\end{array}$ & $\begin{array}{l}90 \cdot 5 \\
(87 \cdot 0-94 \cdot 1)\end{array}$ & $\begin{array}{l}95 \cdot 6 \\
(92 \cdot 9-98 \cdot 2)\end{array}$ \\
\hline $2010-14$ & $\begin{array}{l}86 \cdot 4 \\
(85 \cdot 9-86 \cdot 9)\end{array}$ & $\begin{array}{l}65 \cdot 4 \\
(63 \cdot 5-67 \cdot 2)\end{array}$ & $\begin{array}{l}43 \cdot 1 \\
(41 \cdot 6-44 \cdot 6)\end{array}$ & $\begin{array}{l}93 \cdot 8 \\
(93 \cdot 2-94 \cdot 3)\end{array}$ & $\begin{array}{l}31 \cdot 2 \\
(29 \cdot 9-32 \cdot 5)\end{array}$ & $\begin{array}{l}55 \cdot 4 \\
(54 \cdot 2-56 \cdot 5)\end{array}$ & $\begin{array}{l}70 \cdot 6 \\
(69 \cdot 8-71 \cdot 4)\end{array}$ & $\begin{array}{l}74 \cdot 5 \\
(70 \cdot 2-78 \cdot 9)\end{array}$ & $\begin{array}{l}90 \cdot 8 \\
(87 \cdot 3-94 \cdot 3)\end{array}$ & $\begin{array}{l}95 \cdot 4 \\
(92 \cdot 7-98 \cdot 1)\end{array}$ \\
\hline \multicolumn{11}{|l|}{ Bulgaria* } \\
\hline $2000-04$ & $\begin{array}{l}70 \cdot 9 \\
(69 \cdot 7-72 \cdot 1)\end{array}$ & $\begin{array}{l}49 \cdot 2 \\
(47 \cdot 6-50 \cdot 7)\end{array}$ & $\begin{array}{l}32 \cdot 9 \\
(30 \cdot 9-34 \cdot 9)\end{array}$ & $\begin{array}{l}49 \cdot 4 \\
(47 \cdot 1-51 \cdot 7)\end{array}$ &.. & $\begin{array}{l}29 \cdot 9 \S \\
(27 \cdot 2-32 \cdot 6)\end{array}$ & $\begin{array}{l}38 \cdot 1 \\
(36 \cdot 1-40 \cdot 0)\end{array}$ &.. & $\begin{array}{l}63 \cdot 5 \\
(55 \cdot 5-71 \cdot 5)\end{array}$ & $\begin{array}{c}70 \cdot 7 \\
(62 \cdot 2-79 \cdot 2)\end{array}$ \\
\hline 2005-09 & $\begin{array}{l}75 \cdot 9 \\
(74 \cdot 8-77 \cdot 0)\end{array}$ & $\begin{array}{l}53 \cdot 2 \\
(51 \cdot 7-54 \cdot 7)\end{array}$ & $\begin{array}{l}33 \cdot 9 \\
(32 \cdot 2-35 \cdot 5)\end{array}$ & $\begin{array}{l}54 \cdot 8 \\
(52 \cdot 7-56 \cdot 9)\end{array}$ &.$\cdot$ & $\begin{array}{l}40 \cdot 1 \\
(37 \cdot 6-42 \cdot 6)\end{array}$ & $\begin{array}{l}39 \cdot 9 \\
(38 \cdot 1-41 \cdot 6)\end{array}$ &.. & $\begin{array}{l}74 \cdot 8 \\
(68 \cdot 4-81 \cdot 1)\end{array}$ & $\begin{array}{c}74 \cdot 3 \\
(64 \cdot 9-83 \cdot 7)\end{array}$ \\
\hline 2010-14 & $\begin{array}{l}78 \cdot 3 \\
(77 \cdot 2-79 \cdot 4)\end{array}$ & $\begin{array}{l}54 \cdot 8 \\
(53 \cdot 3-56 \cdot 3)\end{array}$ & $\begin{array}{l}37 \cdot 3 \\
(35 \cdot 4-39 \cdot 1)\end{array}$ & $\begin{array}{l}68 \cdot 3 \\
(66 \cdot 2-70 \cdot 5)\end{array}$ &.$\cdot$ & $\begin{array}{l}41 \cdot 6 \$ \\
(39 \cdot 2-44 \cdot 1)\end{array}$ & $\begin{array}{l}43 \cdot 5 \\
(41 \cdot 8-45 \cdot 2)\end{array}$ &. & $\begin{array}{l}78 \cdot 3 \\
(71 \cdot 7-84 \cdot 9)\end{array}$ & $\begin{array}{c}87 \cdot 7 \\
(80 \cdot 6-94 \cdot 9)\end{array}$ \\
\hline \multicolumn{11}{|l|}{ Croatia* } \\
\hline $2000-04$ & $\begin{array}{l}73 \cdot 6 \\
(72 \cdot 2-75 \cdot 0)\end{array}$ & $\begin{array}{l}63 \cdot 2 \\
(60 \cdot 6-65 \cdot 8)\end{array}$ & $\begin{array}{l}36 \cdot 9 \\
(34 \cdot 6-39 \cdot 2)\end{array}$ & $\begin{array}{l}65 \cdot 7 \\
(63.6-67.9)\end{array}$ & $\begin{array}{l}37 \cdot 0 \\
(35 \cdot 1-38 \cdot 8)\end{array}$ & $\begin{array}{l}31 \cdot 1 \\
(28 \cdot 6-33 \cdot 6)\end{array}$ & $\begin{array}{l}42 \cdot 7 \\
(41 \cdot 0-44 \cdot 4)\end{array}$ & $\begin{array}{l}65 \cdot 7 \\
(58 \cdot 3-73 \cdot 1)\end{array}$ & $\begin{array}{l}81 \cdot 7 \\
(74 \cdot 3-89 \cdot 1)\end{array}$ & $\begin{array}{c}76 \cdot 6 \\
(66 \cdot 8-86 \cdot 3)\end{array}$ \\
\hline 2005-09 & $\begin{array}{l}78 \cdot 2 \\
(77 \cdot 0-79 \cdot 4)\end{array}$ & $\begin{array}{l}64 \cdot 3 \\
(61 \cdot 9-66 \cdot 7)\end{array}$ & $\begin{array}{l}33 \cdot 4 \\
(31 \cdot 3-35 \cdot 5)\end{array}$ & $\begin{array}{l}78 \cdot 3 \\
(76 \cdot 7-79 \cdot 9)\end{array}$ & $\begin{array}{l}39 \cdot 6 \\
(37 \cdot 8-41 \cdot 5)\end{array}$ & $\begin{array}{l}29 \cdot 2 \\
(26 \cdot 8-31 \cdot 6)\end{array}$ & $\begin{array}{l}51 \cdot 0 \\
(49 \cdot 3-52 \cdot 6)\end{array}$ & $\begin{array}{l}75 \cdot 2 \\
(67 \cdot 9-82 \cdot 5)\end{array}$ & $\begin{array}{l}86 \cdot 7 \\
(81 \cdot 1-92 \cdot 3)\end{array}$ & $\begin{array}{c}84 \cdot 6 \\
(76 \cdot 2-93 \cdot 0)\end{array}$ \\
\hline 2010-14 & $\begin{array}{l}78 \cdot 6 \\
(77 \cdot 4-79 \cdot 7)\end{array}$ & $\begin{array}{l}63 \cdot 2 \\
(60 \cdot 8-65 \cdot 6)\end{array}$ & $\begin{array}{l}36 \cdot 0 \\
(33 \cdot 9-38 \cdot 2)\end{array}$ & $\begin{array}{l}80 \cdot 9 \\
(79 \cdot 3-82 \cdot 4)\end{array}$ & $\begin{array}{l}42 \cdot 2 \\
(40 \cdot 4-44 \cdot 0)\end{array}$ & $\begin{array}{l}32 \cdot 2 \\
(29 \cdot 7-34 \cdot 7)\end{array}$ & $\begin{array}{l}52 \cdot 7 \\
(51 \cdot 1-54 \cdot 3)\end{array}$ & $\begin{array}{l}73 \cdot 4 \\
(65 \cdot 6-81 \cdot 3)\end{array}$ & $\begin{array}{l}85 \cdot 2 \\
(79 \cdot 1-91 \cdot 3)\end{array}$ & $\begin{array}{c}94 \cdot 5 \\
(89 \cdot 1-99 \cdot 9)\end{array}$ \\
\hline \multicolumn{11}{|c|}{ Czech Republic* } \\
\hline $2000-04$ & $\begin{array}{l}75 \cdot 7 \\
(74 \cdot 8-76 \cdot 5)\end{array}$ & $\begin{array}{l}60 \cdot 4 \\
(58 \cdot 9-61 \cdot 9)\end{array}$ & $\begin{array}{l}34 \cdot 3 \\
(33 \cdot 0-35 \cdot 5)\end{array}$ & $\begin{array}{l}71 \cdot 0 \\
(69 \cdot 9-72 \cdot 2)\end{array}$ & $\begin{array}{l}19 \cdot 5 \\
(18 \cdot 2-20 \cdot 8)\end{array}$ & $\begin{array}{l}19 \cdot 9 \\
(18 \cdot 2-21 \cdot 7)\end{array}$ & $\begin{array}{l}49 \cdot 9 \\
(48 \cdot 8-51 \cdot 0)\end{array}$ & $\begin{array}{l}55 \cdot 6 \\
(47 \cdot 9-63 \cdot 3)\end{array}$ & $\begin{array}{l}88 \cdot 0 \\
(82 \cdot 9-93 \cdot 1)\end{array}$ & $\begin{array}{c}89 \cdot 0 \\
(82 \cdot 7-95 \cdot 3)\end{array}$ \\
\hline 2005-09 & $\begin{array}{l}79 \cdot 1 \\
(78 \cdot 4-79 \cdot 9)\end{array}$ & $\begin{array}{l}62 \cdot 7 \\
(61 \cdot 2-64 \cdot 1)\end{array}$ & $\begin{array}{l}35 \cdot 3 \\
(34 \cdot 0-36 \cdot 5)\end{array}$ & $\begin{array}{l}81 \cdot 5 \\
(80 \cdot 7-82 \cdot 3)\end{array}$ & $\begin{array}{l}22 \cdot 1 \\
(20 \cdot 8-23 \cdot 4)\end{array}$ & $\begin{array}{l}33 \cdot 5 \\
(31 \cdot 8-35 \cdot 1)\end{array}$ & $\begin{array}{l}53 \cdot 2 \\
(52 \cdot 2-54 \cdot 3)\end{array}$ & $\begin{array}{l}64 \cdot 3 \\
(57 \cdot 4-71 \cdot 3)\end{array}$ & $\begin{array}{l}90 \cdot 3 \\
(85 \cdot 6-95 \cdot 0)\end{array}$ & $\begin{array}{c}82 \cdot 9 \\
(73 \cdot 2-92 \cdot 5)\end{array}$ \\
\hline \multirow[t]{2}{*}{ 2010-14 } & $\begin{array}{l}81 \cdot 4 \\
(80 \cdot 7-82 \cdot 1)\end{array}$ & $\begin{array}{l}61 \cdot 0 \\
(59 \cdot 5-62 \cdot 4)\end{array}$ & $\begin{array}{l}36 \cdot 5 \\
(35 \cdot 2-37 \cdot 8)\end{array}$ & $\begin{array}{l}85 \cdot 3 \\
(84 \cdot 6-86 \cdot 0)\end{array}$ & $\begin{array}{l}21 \cdot 4 \\
(20 \cdot 1-22 \cdot 7)\end{array}$ & $\begin{array}{l}36 \cdot 8 \\
(35 \cdot 2-38 \cdot 5)\end{array}$ & $\begin{array}{l}57 \cdot 2 \\
(56 \cdot 1-58 \cdot 2)\end{array}$ & $\begin{array}{l}69 \cdot 7 \\
(63 \cdot 2-76 \cdot 2)\end{array}$ & $\begin{array}{l}88 \cdot 2 \\
(82 \cdot 1-94 \cdot 3)\end{array}$ & $\begin{array}{c}89.6 \\
(82 \cdot 5-96 \cdot 6)\end{array}$ \\
\hline & & & & & & & & & \multicolumn{2}{|c|}{ (Table 7 continues on next page) } \\
\hline
\end{tabular}




\begin{tabular}{|c|c|c|c|c|c|c|c|c|c|c|}
\hline & \multicolumn{3}{|c|}{ Women's cancers } & \multirow[t]{2}{*}{ Prostate } & \multirow[t]{2}{*}{$\begin{array}{l}\text { Brain } \\
\text { (adults) }\end{array}$} & \multicolumn{2}{|c|}{$\begin{array}{l}\text { Haemopoietic malignancies } \\
\text { (adults) }\end{array}$} & \multicolumn{3}{|c|}{ Childhood malignancies } \\
\hline & Breast & Cervix & Ovary & & & Myeloid & Lymphoid & Brain & $\begin{array}{l}\text { Acute } \\
\text { lymphoblastic } \\
\text { leukaemia }\end{array}$ & Lymphoma \\
\hline \multicolumn{11}{|c|}{ (Continued from previous page) } \\
\hline \multicolumn{11}{|l|}{ Denmark* $^{*}$} \\
\hline 2000-04 & $\begin{array}{l}80 \cdot 3 \\
(79 \cdot 4-81 \cdot 2)\end{array}$ & $\begin{array}{l}63 \cdot 0 \\
(60 \cdot 6-65 \cdot 4)\end{array}$ & $\begin{array}{l}33 \cdot 1 \\
(31 \cdot 4-34 \cdot 8)\end{array}$ & $\begin{array}{l}63 \cdot 6 \\
(62 \cdot 1-65 \cdot 1)\end{array}$ & $\begin{array}{l}30 \cdot 5 \\
(28 \cdot 8-32 \cdot 2)\end{array}$ & $\begin{array}{l}35 \cdot 7 \\
(33 \cdot 6-37 \cdot 8)\end{array}$ & $\begin{array}{l}57 \cdot 7 \\
(56 \cdot 4-59 \cdot 0)\end{array}$ & $\begin{array}{l}68 \cdot 8 \\
(62 \cdot 1-75 \cdot 5)\end{array}$ & $\begin{array}{l}84 \cdot 3 \\
(78 \cdot 6-90 \cdot 0)\end{array}$ & $\begin{array}{c}90 \cdot 2 \\
(82 \cdot 2-98 \cdot 2)\end{array}$ \\
\hline 2005-09 & $\begin{array}{l}84 \cdot 0 \\
(83 \cdot 2-84 \cdot 8)\end{array}$ & $\begin{array}{l}66 \cdot 7 \\
(64 \cdot 3-69 \cdot 1)\end{array}$ & $\begin{array}{l}37 \cdot 4 \\
(35 \cdot 7-39 \cdot 2)\end{array}$ & $\begin{array}{l}82 \cdot 5 \\
(81 \cdot 6-83 \cdot 4)\end{array}$ & $\begin{array}{l}35 \cdot 1 \\
(33 \cdot 4-36 \cdot 7)\end{array}$ & $\begin{array}{l}45 \cdot 0 \\
(43 \cdot 0-47 \cdot 0)\end{array}$ & $\begin{array}{l}66 \cdot 9 \\
(65 \cdot 7-68 \cdot 0)\end{array}$ & $\begin{array}{l}79 \cdot 4 \\
(72 \cdot 9-85 \cdot 8)\end{array}$ & $\begin{array}{l}93 \cdot 1 \\
(88 \cdot 4-97 \cdot 7)\end{array}$ & $\begin{array}{c}90 \cdot 0 \\
(83 \cdot 1-97 \cdot 0)\end{array}$ \\
\hline 2010-14 & $\begin{array}{l}86 \cdot 1 \\
(85 \cdot 4-86 \cdot 9)\end{array}$ & $\begin{array}{l}69 \cdot 5 \\
(67 \cdot 0-72 \cdot 0)\end{array}$ & $\begin{array}{l}39 \cdot 7 \\
(37 \cdot 8-41 \cdot 6)\end{array}$ & $\begin{array}{l}85 \cdot 6 \\
(84 \cdot 7-86 \cdot 4)\end{array}$ & $\begin{array}{l}38 \cdot 9 \\
(37 \cdot 3-40 \cdot 6)\end{array}$ & $\begin{array}{l}47 \cdot 6 \\
(45 \cdot 7-49 \cdot 6)\end{array}$ & $\begin{array}{l}70 \cdot 9 \\
(69 \cdot 8-72 \cdot 1)\end{array}$ & $\begin{array}{l}79 \cdot 5 \\
(73 \cdot 3-85 \cdot 6)\end{array}$ & $\begin{array}{l}94 \cdot 0 \\
(90 \cdot 1-97 \cdot 9)\end{array}$ & $\begin{array}{c}93.8 \\
(89 \cdot 0-98 \cdot 7)\end{array}$ \\
\hline \multicolumn{11}{|l|}{ Estonia* } \\
\hline 2000-04 & $\begin{array}{l}70 \cdot 9 \\
(68 \cdot 2-73 \cdot 6)\end{array}$ & $\begin{array}{l}62 \cdot 3 \\
(58 \cdot 5-66 \cdot 1)\end{array}$ & $\begin{array}{l}31 \cdot 2 \\
(27 \cdot 7-34 \cdot 6)\end{array}$ & $\begin{array}{l}67 \cdot 9 \\
(64 \cdot 5-71 \cdot 3)\end{array}$ & $\begin{array}{l}25 \cdot 2 \\
(21 \cdot 4-29 \cdot 0)\end{array}$ & $\begin{array}{l}36 \cdot 6 \\
(31 \cdot 6-41 \cdot 6)\end{array}$ & $\begin{array}{l}48 \cdot 8 \\
(45 \cdot 6-52 \cdot 0)\end{array}$ & $\begin{array}{l}81 \cdot 2 \\
(69 \cdot 9-92 \cdot 5)\end{array}$ & $\begin{array}{l}63.7 \\
(51.0-76.4)\end{array}$ & $\begin{array}{c}88 \cdot 3 \dagger \\
(73 \cdot 4-100 \cdot 0)\end{array}$ \\
\hline 2005-09 & $\begin{array}{l}75 \cdot 4 \\
(73 \cdot 1-77 \cdot 6)\end{array}$ & $\begin{array}{l}66 \cdot 9 \\
(63 \cdot 4-70 \cdot 4)\end{array}$ & $\begin{array}{l}37 \cdot 2 \\
(33 \cdot 8-40 \cdot 7)\end{array}$ & $\begin{array}{l}83 \cdot 2 \\
(80 \cdot 9-85 \cdot 6)\end{array}$ & $\begin{array}{l}26 \cdot 1 \\
(22 \cdot 6-29 \cdot 6)\end{array}$ & $\begin{array}{l}42 \cdot 4 \\
(38 \cdot 1-46 \cdot 8)\end{array}$ & $\begin{array}{l}53 \cdot 5 \\
(50 \cdot 6-56 \cdot 4)\end{array}$ & $\begin{array}{l}72 \cdot 3^{\dagger} \\
(57 \cdot 9-86 \cdot 7)\end{array}$ & $\begin{array}{l}85 \cdot 4 \dagger \\
(73 \cdot 6-97 \cdot 1)\end{array}$ & $\begin{array}{c}85 \cdot 8 \dagger \\
(68 \cdot 1-100 \cdot 0)\end{array}$ \\
\hline 2010-14 & $\begin{array}{l}76 \cdot 6 \\
(73 \cdot 8-79 \cdot 3)\end{array}$ & $\begin{array}{l}66 \cdot 5 \\
(62 \cdot 2-70 \cdot 7)\end{array}$ & $\begin{array}{l}42 \cdot 3 \\
(37 \cdot 4-47 \cdot 1)\end{array}$ & $\begin{array}{l}86 \cdot 3 \\
(83 \cdot 5-89 \cdot 0)\end{array}$ & $\begin{array}{l}31 \cdot 0 \\
(26 \cdot 1-36 \cdot 0)\end{array}$ & $\begin{array}{l}37 \cdot 8 \\
(32 \cdot 1-43 \cdot 5)\end{array}$ & $\begin{array}{l}53 \cdot 8 \\
(50 \cdot 2-57 \cdot 4)\end{array}$ & $\begin{array}{l}64 \cdot 5 \\
(47 \cdot 3-81 \cdot 8)\end{array}$ & $\begin{array}{l}87.7 \\
(76 \cdot 4-98 \cdot 9)\end{array}$ & $\begin{array}{c}88 \cdot 0 \\
(73 \cdot 1-100 \cdot 0)\end{array}$ \\
\hline \multicolumn{11}{|l|}{ Finland* } \\
\hline $2000-04$ & $\begin{array}{l}86 \cdot 5 \\
(85 \cdot 5-87 \cdot 5)\end{array}$ & $\begin{array}{l}67 \cdot 8 \\
(64 \cdot 3-71 \cdot 3)\end{array}$ & $\begin{array}{l}41 \cdot 2 \\
(39 \cdot 2-43 \cdot 1)\end{array}$ & $\begin{array}{l}90 \cdot 0 \\
(89.1-90 \cdot 9)\end{array}$ & $\begin{array}{l}35 \cdot 4 \\
(33 \cdot 5-37 \cdot 4)\end{array}$ & $\begin{array}{l}40 \cdot 15 \\
(37 \cdot 4-42 \cdot 8)\end{array}$ & $\begin{array}{l}56 \cdot 8 \\
(55 \cdot 5-58 \cdot 1)\end{array}$ & $\begin{array}{l}78 \cdot 5 \\
(72 \cdot 5-84 \cdot 6)\end{array}$ & $\begin{array}{l}84 \cdot 7 \\
(78 \cdot 0-91 \cdot 4)\end{array}$ & $\begin{array}{c}90 \cdot 5 \\
(84 \cdot 3-96 \cdot 7)\end{array}$ \\
\hline $2005-09$ & $\begin{array}{l}87 \cdot 7 \\
(86 \cdot 9-88 \cdot 5)\end{array}$ & $\begin{array}{l}65 \cdot 2 \\
(61 \cdot 4-69 \cdot 0)\end{array}$ & $\begin{array}{l}44 \cdot 2 \\
(42 \cdot 2-46 \cdot 2)\end{array}$ & $\begin{array}{l}93 \cdot 4 \\
(92 \cdot 6-94 \cdot 1)\end{array}$ & $\begin{array}{l}35 \cdot 8 \\
(34 \cdot 0-37 \cdot 6)\end{array}$ & $\begin{array}{l}41 \cdot 6 \$ \\
(39 \cdot 2-44 \cdot 0)\end{array}$ & $\begin{array}{l}62 \cdot 5 \\
(61 \cdot 2-63 \cdot 7)\end{array}$ & $\begin{array}{l}78 \cdot 0 \\
(71 \cdot 5-84.5)\end{array}$ & $\begin{array}{l}88 \cdot 1 \\
(83 \cdot 2-92 \cdot 9)\end{array}$ & $\begin{array}{c}92 \cdot 0 \\
(84 \cdot 5-99 \cdot 6)\end{array}$ \\
\hline 2010-14 & $\begin{array}{l}88 \cdot 5 \\
(87 \cdot 7-89 \cdot 3)\end{array}$ & $\begin{array}{l}67 \cdot 4 \\
(63 \cdot 8-71 \cdot 1)\end{array}$ & $\begin{array}{l}41 \cdot 1 \\
(39 \cdot 2-43 \cdot 0)\end{array}$ & $\begin{array}{l}93 \cdot 2 \\
(92 \cdot 4-93 \cdot 9)\end{array}$ & $\begin{array}{l}37 \cdot 6 \\
(35 \cdot 7-39 \cdot 4)\end{array}$ & $\begin{array}{l}47 \cdot 25 \\
(44 \cdot 7-49 \cdot 6)\end{array}$ & $\begin{array}{l}64 \cdot 4 \\
(63 \cdot 2-65 \cdot 5)\end{array}$ & $\begin{array}{l}75 \cdot 6 \\
(69 \cdot 0-82 \cdot 2)\end{array}$ & $\begin{array}{l}95 \cdot 2 \\
(91 \cdot 5-98 \cdot 9)\end{array}$ & $\begin{array}{c}91 \cdot 2 \\
(84 \cdot 3-98 \cdot 1)\end{array}$ \\
\hline \multicolumn{11}{|c|}{ France (23 registries) $\ddagger$} \\
\hline 2000-04 & $\begin{array}{l}86 \cdot 8 \\
(86 \cdot 1-87 \cdot 4)\end{array}$ & $\begin{array}{l}61 \cdot 7 \\
(59 \cdot 4-64 \cdot 0)\end{array}$ & $\begin{array}{l}41 \cdot 6 \\
(39 \cdot 8-43 \cdot 3)\end{array}$ & $\begin{array}{l}90 \cdot 1 \\
(89 \cdot 4-90 \cdot 8)\end{array}$ & $\begin{array}{l}21 \cdot 8 \\
(20 \cdot 3-23 \cdot 3)\end{array}$ & $\begin{array}{l}53 \cdot 3 \\
(51 \cdot 9-54 \cdot 7)\end{array}$ & $\begin{array}{l}64 \cdot 4 \\
(63 \cdot 6-65 \cdot 3)\end{array}$ & $\begin{array}{l}67 \cdot 2 \\
(65 \cdot 0-69 \cdot 5)\end{array}$ & $\begin{array}{l}88 \cdot 1 \\
(86 \cdot 4-89 \cdot 8)\end{array}$ & $\begin{array}{c}92 \cdot 7 \\
(90 \cdot 8-94 \cdot 6)\end{array}$ \\
\hline 2005-09 & $\begin{array}{l}87 \cdot 2 \\
(86 \cdot 6-87 \cdot 8)\end{array}$ & $\begin{array}{l}62 \cdot 1 \\
(60 \cdot 0-64 \cdot 2)\end{array}$ & $\begin{array}{l}42 \cdot 1 \\
(40 \cdot 4-43 \cdot 7)\end{array}$ & $\begin{array}{l}93 \cdot 6 \\
(93 \cdot 1-94 \cdot 1)\end{array}$ & $\begin{array}{l}27 \cdot 2 \\
(25 \cdot 8-28 \cdot 7)\end{array}$ & $\begin{array}{l}54 \cdot 6 \\
(53 \cdot 3-55 \cdot 8)\end{array}$ & $\begin{array}{l}69 \cdot 9 \\
(69 \cdot 1-70 \cdot 7)\end{array}$ & $\begin{array}{l}70 \cdot 8 \\
(68 \cdot 6-73 \cdot 0)\end{array}$ & $\begin{array}{l}90 \cdot 0 \\
(88 \cdot 4-91 \cdot 6)\end{array}$ & $\begin{array}{l}92 \cdot 6 \\
(90 \cdot 6-94 \cdot 6)\end{array}$ \\
\hline 2010-14 & $\begin{array}{l}86.7 \\
(85 \cdot 5-88.0)\end{array}$ & $\begin{array}{l}65 \cdot 0 \\
(60 \cdot 3-69 \cdot 7)\end{array}$ & $\begin{array}{l}43 \cdot 5 \\
(40 \cdot 0-46 \cdot 9)\end{array}$ & $\begin{array}{l}93 \cdot 1 \\
(91 \cdot 9-94 \cdot 2)\end{array}$ & $\begin{array}{l}27 \cdot 2 \\
(24 \cdot 2-30 \cdot 3)\end{array}$ & $\begin{array}{l}57 \cdot 5 \\
(54.9-60 \cdot 1)\end{array}$ & $\begin{array}{l}69 \cdot 6 \\
(67 \cdot 9-71 \cdot 3)\end{array}$ & $\begin{array}{l}70 \cdot 8 \\
(68 \cdot 1-73 \cdot 5)\end{array}$ & $\begin{array}{l}88 \cdot 6 \\
(86 \cdot 5-90 \cdot 8)\end{array}$ & $\begin{array}{c}94 \cdot 2 \\
(92 \cdot 0-96 \cdot 4)\end{array}$ \\
\hline \multicolumn{11}{|c|}{ Germany (ten registries) } \\
\hline 2000-04 & $\begin{array}{l}83 \cdot 9 \\
(83 \cdot 4-84 \cdot 4)\end{array}$ & $\begin{array}{l}64 \cdot 9 \\
(63 \cdot 8-66 \cdot 1)\end{array}$ & $\begin{array}{l}40 \cdot 8 \\
(39 \cdot 3-42 \cdot 2)\end{array}$ & $\begin{array}{l}90 \cdot 4 \\
(89.9-90 \cdot 9)\end{array}$ & $\begin{array}{l}29 \cdot 1 \\
(24 \cdot 8-33 \cdot 3)\end{array}$ & $\begin{array}{l}46 \cdot 0 \\
(43 \cdot 9-48 \cdot 1)\end{array}$ & $\begin{array}{l}61 \cdot 2 \\
(60 \cdot 4-62 \cdot 0)\end{array}$ & $\begin{array}{l}63 \cdot 1 \\
(56 \cdot 0-70 \cdot 2)\end{array}$ & $\begin{array}{l}94 \cdot 0 \\
(90 \cdot 7-97 \cdot 3)\end{array}$ & $\begin{array}{c}90 \cdot 4 \\
(84 \cdot 2-96 \cdot 5)\end{array}$ \\
\hline 2005-09 & $\begin{array}{l}85 \cdot 6 \\
(85 \cdot 2-85 \cdot 9)\end{array}$ & $\begin{array}{l}65 \cdot 7 \\
(64 \cdot 6-66 \cdot 8)\end{array}$ & $\begin{array}{l}40 \cdot 6 \\
(39 \cdot 6-41 \cdot 6)\end{array}$ & $\begin{array}{l}91 \cdot 8 \\
(91 \cdot 3-92 \cdot 2)\end{array}$ & $\begin{array}{l}27 \cdot 3 \\
(26 \cdot 2-28 \cdot 5)\end{array}$ & $\begin{array}{l}51 \cdot 9 \\
(50 \cdot 7-53 \cdot 1)\end{array}$ & $\begin{array}{l}65 \cdot 7 \\
(65 \cdot 1-66 \cdot 3)\end{array}$ & $\begin{array}{l}67 \cdot 8 \\
(61 \cdot 1-74 \cdot 6)\end{array}$ & $\begin{array}{l}92 \cdot 1 \\
(89 \cdot 2-95 \cdot 0)\end{array}$ & $\begin{array}{c}93.8 \\
(88.9-98.8)\end{array}$ \\
\hline 2010-14 & $\begin{array}{l}86 \cdot 0 \\
(85 \cdot 7-86 \cdot 4)\end{array}$ & $\begin{array}{l}65 \cdot 2 \\
(64 \cdot 0-66 \cdot 4)\end{array}$ & $\begin{array}{l}41 \cdot 2 \\
(40 \cdot 2-42 \cdot 2)\end{array}$ & $\begin{array}{l}91 \cdot 6 \\
(91 \cdot 2-92 \cdot 0)\end{array}$ & $\begin{array}{l}29 \cdot 6 \\
(28 \cdot 3-30 \cdot 9)\end{array}$ & $\begin{array}{l}54 \cdot 9 \\
(53 \cdot 5-56 \cdot 3)\end{array}$ & $\begin{array}{l}67 \cdot 9 \\
(67 \cdot 3-68 \cdot 6)\end{array}$ & $\begin{array}{l}69 \cdot 5 \\
(61 \cdot 8-77 \cdot 2)\end{array}$ & $\begin{array}{l}91 \cdot 1 \\
(87 \cdot 4-94 \cdot 8)\end{array}$ & $\begin{array}{c}96 \cdot 9 \\
(94 \cdot 2-99 \cdot 6)\end{array}$ \\
\hline \multicolumn{11}{|l|}{ Gibraltar* } \\
\hline $2000-04$ & $\begin{array}{l}79 \cdot 3+5 \\
(66 \cdot 5-92 \cdot 1)\end{array}$ & .. &.. & $\begin{array}{l}62 \cdot 5+5 \\
(31 \cdot 5-93 \cdot 5)\end{array}$ & .. &.. & .. & .. & .. & .. \\
\hline 2005-09 & $\begin{array}{l}72 \cdot 15 \\
(56 \cdot 8-87 \cdot 4)\end{array}$ & .. &.. & $\begin{array}{l}42 \cdot 3+5 \\
(9 \cdot 1-75 \cdot 6)\end{array}$ & .. &. & .. & .. & .. & .. \\
\hline 2010-14 & .. & .. & .. & .. & .. &.$\cdot$ & .. & .. & .. &.$\cdot$ \\
\hline \multicolumn{11}{|c|}{ Greece (childhood) $\ddagger$} \\
\hline $2000-04$ & .. & .. & .. &.$\cdot$ &.$\cdot$ &.$\cdot$ & .. & .. & $\begin{array}{l}80 \cdot 3 \\
(75 \cdot 3-85 \cdot 4)\end{array}$ & $\begin{array}{c}89 \cdot 2 \\
(82 \cdot 6-95 \cdot 8)\end{array}$ \\
\hline 2005-09 &. & .. &.. &.. & .. &.. & .. & .. & $\begin{array}{l}84 \cdot 8 \\
(80 \cdot 4-89 \cdot 3)\end{array}$ & $\begin{array}{c}84 \cdot 9 \\
(78 \cdot 3-91 \cdot 6)\end{array}$ \\
\hline \multirow[t]{2}{*}{ 2010-14 } &. & .. & .. &.. &.. &. &.. & $\begin{array}{l}68 \cdot 9 \\
(60 \cdot 3-77 \cdot 4)\end{array}$ & $\begin{array}{l}84 \cdot 2 \\
(80 \cdot 0-88 \cdot 3)\end{array}$ & $\begin{array}{c}87 \cdot 5 \\
(81 \cdot 5-93 \cdot 5)\end{array}$ \\
\hline & & & & & & & & & \multicolumn{2}{|c|}{ (Table 7 continues on next page } \\
\hline
\end{tabular}




\begin{tabular}{|c|c|c|c|c|c|c|c|c|c|c|}
\hline & \multicolumn{3}{|c|}{ Women's cancers } & \multirow[t]{2}{*}{ Prostate } & \multirow[t]{2}{*}{$\begin{array}{l}\text { Brain } \\
\text { (adults) }\end{array}$} & \multicolumn{2}{|c|}{$\begin{array}{l}\text { Haemopoietic malignancies } \\
\text { (adults) }\end{array}$} & \multicolumn{3}{|c|}{ Childhood malignancies } \\
\hline & Breast & Cervix & Ovary & & & Myeloid & Lymphoid & Brain & $\begin{array}{l}\text { Acute } \\
\text { lymphoblastic } \\
\text { leukaemia }\end{array}$ & Lymphoma \\
\hline \multicolumn{11}{|c|}{ (Continued from previous page) } \\
\hline \multicolumn{11}{|l|}{ Iceland* } \\
\hline 2000-04 & $\begin{array}{l}87 \cdot 4 \\
(82 \cdot 9-91 \cdot 8)\end{array}$ & $\begin{array}{l}81 \cdot 8 \dagger \\
(72 \cdot 8-90 \cdot 8)\end{array}$ & $\begin{array}{l}39 \cdot 6 \\
(30 \cdot 9-48 \cdot 3)\end{array}$ & $\begin{array}{l}80 \cdot 1 \\
(75 \cdot 5-84 \cdot 7)\end{array}$ & $\begin{array}{l}24 \cdot 1 \\
(19 \cdot 5-28 \cdot 6)\end{array}$ & $\begin{array}{l}48 \cdot 2 \\
(38 \cdot 3-58 \cdot 1)\end{array}$ & $\begin{array}{l}66 \cdot 6 \\
(60 \cdot 4-72 \cdot 8)\end{array}$ & $\begin{array}{l}80 \cdot 0 \dagger \\
(56 \cdot 6-100 \cdot 0)\end{array}$ & & .. \\
\hline 2005-09 & $\begin{array}{l}85 \cdot 8 \\
(81 \cdot 4-90 \cdot 2)\end{array}$ & $\begin{array}{l}87 \cdot 6 \dagger \\
(79 \cdot 5-95 \cdot 7)\end{array}$ & $\begin{array}{l}40 \cdot 9 \\
(31 \cdot 3-50 \cdot 5)\end{array}$ & $\begin{array}{l}89.7 \\
(86.5-92 \cdot 9)\end{array}$ & $\begin{array}{l}21 \cdot 0 \\
(14 \cdot 3-27 \cdot 6)\end{array}$ & $\begin{array}{l}47 \cdot 9 \\
(39 \cdot 7-56 \cdot 1)\end{array}$ & $\begin{array}{l}67 \cdot 9 \\
(62 \cdot 6-73 \cdot 1)\end{array}$ & $\begin{array}{l}73 \cdot 4^{\dagger} \\
(51 \cdot 8-94 \cdot 9)\end{array}$ & .. & .. \\
\hline 2010-14 & $\begin{array}{l}89 \cdot 1 \\
(85 \cdot 1-93 \cdot 1)\end{array}$ & $\begin{array}{l}80 \cdot 1 \dagger \\
(71 \cdot 2-89 \cdot 1)\end{array}$ & $\begin{array}{l}40 \cdot 3 \\
(31 \cdot 2-49 \cdot 4)\end{array}$ & $\begin{array}{l}90 \cdot 8 \\
(87 \cdot 7-93 \cdot 9)\end{array}$ & $\begin{array}{l}29 \cdot 2 \\
(20 \cdot 8-37 \cdot 7)\end{array}$ & $\begin{array}{l}43 \cdot 4 \\
(35 \cdot 8-50 \cdot 9)\end{array}$ & $\begin{array}{l}71 \cdot 5 \\
(66 \cdot 3-76 \cdot 6)\end{array}$ & $\begin{array}{l}90 \cdot 1 \dagger \\
(72 \cdot 4-100 \cdot 0)\end{array}$ & $\begin{array}{l}92 \cdot 4 \dagger \\
(78 \cdot 4-100 \cdot 0)\end{array}$ & .. \\
\hline \multicolumn{11}{|l|}{ Ireland* } \\
\hline 2000-04 & $\begin{array}{l}77 \cdot 2 \\
(75 \cdot 8-78 \cdot 5)\end{array}$ & $\begin{array}{l}57 \cdot 8 \\
(54 \cdot 1-61 \cdot 6)\end{array}$ & $\begin{array}{l}29 \cdot 4 \\
(27 \cdot 2-31 \cdot 7)\end{array}$ & $\begin{array}{l}83 \cdot 7 \\
(82 \cdot 5-84 \cdot 9)\end{array}$ & $\begin{array}{l}26 \cdot 9 \\
(24 \cdot 9-29 \cdot 0)\end{array}$ & $\begin{array}{l}47 \cdot 6 \\
(44 \cdot 9-50 \cdot 2)\end{array}$ & $\begin{array}{l}55 \cdot 4 \\
(53 \cdot 8-57 \cdot 0)\end{array}$ & $\begin{array}{l}67 \cdot 8 \\
(60 \cdot 0-75 \cdot 6)\end{array}$ & $\begin{array}{l}82 \cdot 9 \\
(76 \cdot 6-89 \cdot 2)\end{array}$ & $\begin{array}{l}97 \cdot 4 \\
(94 \cdot 6-100 \cdot 0)\end{array}$ \\
\hline 2005-09 & $\begin{array}{l}81 \cdot 4 \\
(80 \cdot 2-82 \cdot 6)\end{array}$ & $\begin{array}{l}58 \cdot 7 \\
(55 \cdot 5-61 \cdot 9)\end{array}$ & $\begin{array}{l}31 \cdot 2 \\
(28 \cdot 9-33 \cdot 4)\end{array}$ & $\begin{array}{l}89 \cdot 7 \\
(88 \cdot 7-90 \cdot 7)\end{array}$ & $\begin{array}{l}30 \cdot 8 \\
(28 \cdot 7-33 \cdot 0)\end{array}$ & $\begin{array}{l}52 \cdot 5 \\
(50 \cdot 1-55 \cdot 0)\end{array}$ & $\begin{array}{l}64 \cdot 2 \\
(62 \cdot 7-65 \cdot 7)\end{array}$ & $\begin{array}{l}68 \cdot 6 \\
(61 \cdot 1-76 \cdot 1)\end{array}$ & $\begin{array}{l}87 \cdot 1 \\
(80 \cdot 9-93 \cdot 4)\end{array}$ & $\begin{array}{l}96 \cdot 2 \\
(92 \cdot 0-100 \cdot 0)\end{array}$ \\
\hline 2010-14 & $\begin{array}{l}82 \cdot 0 \\
(80 \cdot 7-83 \cdot 3)\end{array}$ & $\begin{array}{l}63 \cdot 6 \\
(60 \cdot 1-67 \cdot 2)\end{array}$ & $\begin{array}{l}32 \cdot 8 \\
(30 \cdot 3-35 \cdot 3)\end{array}$ & $\begin{array}{l}91 \cdot 1 \\
(90 \cdot 1-92 \cdot 2)\end{array}$ & $\begin{array}{l}34 \cdot 5 \\
(32 \cdot 0-36 \cdot 9)\end{array}$ & $\begin{array}{l}53 \cdot 1 \\
(50 \cdot 5-55 \cdot 8)\end{array}$ & $\begin{array}{l}66 \cdot 9 \\
(65 \cdot 3-68 \cdot 5)\end{array}$ & $\begin{array}{l}74 \cdot 0 \\
(66 \cdot 4-81 \cdot 5)\end{array}$ & $\begin{array}{l}88 \cdot 3 \\
(82 \cdot 4-94 \cdot 3)\end{array}$ & $\begin{array}{l}95 \cdot 3 \\
(89 \cdot 6-100 \cdot 0)\end{array}$ \\
\hline \multicolumn{11}{|c|}{ Italy (45 registries) } \\
\hline 2000-04 & $\begin{array}{l}84 \cdot 2 \\
(83 \cdot 8-84 \cdot 5)\end{array}$ & $\begin{array}{l}67 \cdot 3 \\
(66 \cdot 0-68 \cdot 7)\end{array}$ & $\begin{array}{l}37 \cdot 7 \\
(36 \cdot 8-38 \cdot 6)\end{array}$ & $\begin{array}{l}87 \cdot 2 \\
(86 \cdot 7-87 \cdot 7)\end{array}$ & $\begin{array}{l}24 \cdot 1 \\
(23 \cdot 2-25 \cdot 1)\end{array}$ & $\begin{array}{l}46 \cdot 4 \\
(45 \cdot 5-47 \cdot 3)\end{array}$ & $\begin{array}{l}58 \cdot 5 \\
(57 \cdot 9-59 \cdot 0)\end{array}$ & $\begin{array}{l}68 \cdot 5 \\
(64.5-72 \cdot 5)\end{array}$ & $\begin{array}{l}82 \cdot 6 \\
(79 \cdot 2-86 \cdot 0)\end{array}$ & $\begin{array}{l}88 \cdot 3 \\
(84 \cdot 9-91 \cdot 8)\end{array}$ \\
\hline 2005-09 & $\begin{array}{l}85 \cdot 9 \\
(85 \cdot 5-86 \cdot 2)\end{array}$ & $\begin{array}{l}67 \cdot 7 \\
(66.5-68.9)\end{array}$ & $\begin{array}{l}39 \cdot 3 \\
(38 \cdot 5-40 \cdot 1)\end{array}$ & $\begin{array}{l}89 \cdot 6 \\
(89 \cdot 2-90 \cdot 0)\end{array}$ & $\begin{array}{l}27 \cdot 0 \\
(26 \cdot 2-27 \cdot 8)\end{array}$ & $\begin{array}{l}49 \cdot 2 \\
(48 \cdot 5-49 \cdot 9)\end{array}$ & $\begin{array}{l}61 \cdot 9 \\
(61 \cdot 5-62 \cdot 4)\end{array}$ & $\begin{array}{l}75 \cdot 5 \\
(72 \cdot 3-78 \cdot 8)\end{array}$ & $\begin{array}{l}89 \cdot 2 \\
(86 \cdot 8-91 \cdot 6)\end{array}$ & $\begin{array}{c}89 \cdot 4 \\
(86 \cdot 5-92 \cdot 4)\end{array}$ \\
\hline 2010-14 & $\begin{array}{l}86 \cdot 0 \\
(85 \cdot 5-86 \cdot 4)\end{array}$ & $\begin{array}{l}66 \cdot 8 \\
(65 \cdot 1-68 \cdot 5)\end{array}$ & $\begin{array}{l}39 \cdot 4 \\
(38 \cdot 3-40 \cdot 5)\end{array}$ & $\begin{array}{l}89.5 \\
(89.0-89.9)\end{array}$ & $\begin{array}{l}28 \cdot 8 \\
(27 \cdot 8-29 \cdot 9)\end{array}$ & $\begin{array}{l}49 \cdot 2 \\
(48 \cdot 2-50 \cdot 2)\end{array}$ & $\begin{array}{l}62 \cdot 6 \\
(61 \cdot 9-63 \cdot 2)\end{array}$ & $\begin{array}{l}74 \cdot 8 \\
(70 \cdot 4-79 \cdot 2)\end{array}$ & $\begin{array}{l}87.8 \\
(84 \cdot 4-91 \cdot 2)\end{array}$ & $\begin{array}{c}91.6 \\
(87 \cdot 4-95 \cdot 7)\end{array}$ \\
\hline \multicolumn{11}{|l|}{ Latvia* } \\
\hline 2000-04 & $\begin{array}{l}79 \cdot 7 \\
(77 \cdot 1-82 \cdot 2)\end{array}$ & $\begin{array}{l}52 \cdot 8 \\
(49 \cdot 0-56 \cdot 6)\end{array}$ & $\begin{array}{l}40 \cdot 3 \\
(36 \cdot 6-44 \cdot 0)\end{array}$ & $\begin{array}{l}69 \cdot 9 \\
(66 \cdot 5-73 \cdot 3)\end{array}$ & $\begin{array}{l}22 \cdot 2 \\
(18 \cdot 8-25 \cdot 7)\end{array}$ & $\begin{array}{l}22 \cdot 2 \\
(18 \cdot 7-25 \cdot 7)\end{array}$ & $\begin{array}{l}59 \cdot 8 \\
(54 \cdot 9-64 \cdot 8)\end{array}$ & $\begin{array}{l}79 \cdot 7 \dagger \\
(67 \cdot 1-92 \cdot 2)\end{array}$ & $\begin{array}{l}80 \cdot 5 \\
(70 \cdot 0-91 \cdot 0)\end{array}$ & .. \\
\hline 2005-09 & $\begin{array}{l}79.9 \\
(77 \cdot 8-82.1)\end{array}$ & $\begin{array}{l}57 \cdot 7 \\
(54 \cdot 2-61 \cdot 2)\end{array}$ & $\begin{array}{l}39 \cdot 8 \\
(36 \cdot 5-43 \cdot 1)\end{array}$ & $\begin{array}{l}88.8 \\
(86.0-91 \cdot 6)\end{array}$ & $\begin{array}{l}23 \cdot 9 \\
(20 \cdot 8-27 \cdot 0)\end{array}$ & $\begin{array}{l}17 \cdot 9 \\
(14 \cdot 4-21 \cdot 4)\end{array}$ & $\begin{array}{l}64 \cdot 7 \\
(60 \cdot 5-68.9)\end{array}$ & $\begin{array}{l}72 \cdot 3 \dagger \\
(52 \cdot 2-92 \cdot 3)\end{array}$ & $\begin{array}{l}77 \cdot 0 \\
(66 \cdot 4-87.7)\end{array}$ &. \\
\hline 2010-14 & $\begin{array}{l}82 \cdot 2 \\
(80 \cdot 3-84 \cdot 2)\end{array}$ & $\begin{array}{l}56 \cdot 0 \\
(52 \cdot 6-59 \cdot 5)\end{array}$ & $\begin{array}{l}45 \cdot 5 \\
(41 \cdot 9-49 \cdot 0)\end{array}$ & $\begin{array}{l}90 \cdot 4 \\
(87 \cdot 6-93 \cdot 2)\end{array}$ & $\begin{array}{l}26 \cdot 1 \\
(22 \cdot 7-29 \cdot 5)\end{array}$ & $\begin{array}{l}21 \cdot 4 \\
(17 \cdot 9-25 \cdot 0)\end{array}$ & $\begin{array}{l}71 \cdot 6 \\
(67 \cdot 8-75 \cdot 4)\end{array}$ & $\begin{array}{l}67 \cdot 1 \\
(51 \cdot 2-83 \cdot 1)\end{array}$ & $\begin{array}{l}84 \cdot 1 \\
(73 \cdot 2-94 \cdot 9)\end{array}$ & .. \\
\hline \multicolumn{11}{|l|}{ Lithuania* } \\
\hline 2000-04 & $\begin{array}{l}64 \cdot 6 \\
(62 \cdot 9-66 \cdot 4)\end{array}$ & $\begin{array}{l}53 \cdot 8 \\
(51 \cdot 6-56 \cdot 0)\end{array}$ & $\begin{array}{l}30 \cdot 2 \\
(28 \cdot 1-32 \cdot 3)\end{array}$ & $\begin{array}{l}75 \cdot 8 \\
(73 \cdot 7-77 \cdot 9)\end{array}$ & $\begin{array}{l}19 \cdot 4 \\
(17 \cdot 2-21 \cdot 6)\end{array}$ & $\begin{array}{l}25 \cdot 4 \\
(22 \cdot 5-28 \cdot 3)\end{array}$ & $\begin{array}{l}43 \cdot 5 \\
(41 \cdot 5-45 \cdot 5)\end{array}$ & $\begin{array}{l}47 \cdot 5 \\
(36 \cdot 2-58 \cdot 8)\end{array}$ & $\begin{array}{l}74 \cdot 3 \\
(65 \cdot 2-83 \cdot 3)\end{array}$ & $\begin{array}{c}86 \cdot 1 \\
(77 \cdot 2-95 \cdot 0)\end{array}$ \\
\hline 2005-09 & $\begin{array}{l}71 \cdot 3 \\
(69 \cdot 6-73 \cdot 1)\end{array}$ & $\begin{array}{l}59 \cdot 1 \\
(56 \cdot 9-61 \cdot 3)\end{array}$ & $\begin{array}{l}31 \cdot 6 \\
(29 \cdot 5-33 \cdot 8)\end{array}$ & $\begin{array}{l}93 \cdot 8 \\
(92 \cdot 6-95 \cdot 1)\end{array}$ & $\begin{array}{l}22 \cdot 0 \\
(19 \cdot 7-24 \cdot 3)\end{array}$ & $\begin{array}{l}47 \cdot 5 \\
(45 \cdot 1-49 \cdot 9)\end{array}$ & $\begin{array}{l}50 \cdot 8 \\
(48 \cdot 9-52 \cdot 7)\end{array}$ & $\begin{array}{l}66 \cdot 6 \\
(53 \cdot 4-79 \cdot 8)\end{array}$ & $\begin{array}{l}67 \cdot 7 \\
(56 \cdot 5-78.8)\end{array}$ & $\begin{array}{c}79.6 \dagger \\
(67.1-92.1)\end{array}$ \\
\hline 2010-14 & $\begin{array}{l}73 \cdot 5 \\
(71 \cdot 3-75 \cdot 7)\end{array}$ & $\begin{array}{l}59 \cdot 2 \\
(56 \cdot 4-62 \cdot 0)\end{array}$ & $\begin{array}{l}35 \cdot 0 \\
(32 \cdot 0-37 \cdot 9)\end{array}$ & $\begin{array}{l}94 \cdot 3 \\
(92 \cdot 7-95 \cdot 8)\end{array}$ & $\begin{array}{l}23 \cdot 45 \\
(20 \cdot 4-26 \cdot 4)\end{array}$ & $\begin{array}{l}52 \cdot 8 \\
(49 \cdot 7-56 \cdot 0)\end{array}$ & $\begin{array}{l}56 \cdot 7 \\
(54 \cdot 0-59 \cdot 3)\end{array}$ & $\begin{array}{l}61 \cdot 8 \\
(44 \cdot 7-78.9)\end{array}$ & $\begin{array}{l}74 \cdot 7 \\
(62 \cdot 8-86 \cdot 5)\end{array}$ & $\begin{array}{l}93 \cdot 7 \\
(85 \cdot 9-100 \cdot 0)\end{array}$ \\
\hline \multicolumn{11}{|l|}{ Malta* } \\
\hline 2000-04 & $\begin{array}{l}79 \cdot 7 \\
(75 \cdot 6-83 \cdot 8)\end{array}$ & $\begin{array}{l}46 \cdot 4 \dagger \\
(30 \cdot 8-62 \cdot 0)\end{array}$ & $\begin{array}{l}39 \cdot 6 \\
(31 \cdot 6-47 \cdot 5)\end{array}$ & $\begin{array}{l}81 \cdot 9 \\
(76 \cdot 6-87 \cdot 2)\end{array}$ & $\begin{array}{l}22 \cdot 3 \\
(15 \cdot 2-29 \cdot 3)\end{array}$ & $\begin{array}{l}32 \cdot 7 \\
(24 \cdot 6-40 \cdot 8)\end{array}$ & $\begin{array}{l}43 \cdot 4 \\
(37 \cdot 8-49 \cdot 0)\end{array}$ & $\begin{array}{l}60.1 \dagger \\
(31.7-88.6)\end{array}$ & $\begin{array}{l}87.5 \dagger \\
(71.8-100.0)\end{array}$ & .. \\
\hline 2005-09 & $\begin{array}{l}84 \cdot 8 \\
(81 \cdot 3-88 \cdot 2)\end{array}$ & $\begin{array}{l}65.1 \dagger \\
(51 \cdot 4-78.9)\end{array}$ & $\begin{array}{l}27 \cdot 5 \\
(22 \cdot 0-33 \cdot 0)\end{array}$ & $\begin{array}{l}86 \cdot 4 \\
(81 \cdot 9-91 \cdot 0)\end{array}$ & $\begin{array}{l}20 \cdot 1 \\
(14 \cdot 0-26 \cdot 1)\end{array}$ & $\begin{array}{l}25 \cdot 4 \Omega \\
(18 \cdot 0-32 \cdot 8)\end{array}$ & $\begin{array}{l}52 \cdot 4 \\
(47 \cdot 3-57 \cdot 6)\end{array}$ & .. & $\begin{array}{l}93 \cdot 8 \dagger \\
(82 \cdot 3-100 \cdot 0)\end{array}$ & .. \\
\hline 2010-14 & $\begin{array}{l}86 \cdot 9 \\
(83 \cdot 1-90 \cdot 6)\end{array}$ & $\begin{array}{l}57 \cdot 4 \\
(46 \cdot 8-68 \cdot 1)\end{array}$ & $\begin{array}{l}28 \cdot 0 \\
(21 \cdot 4-34 \cdot 6)\end{array}$ & $\begin{array}{l}88 \cdot 2 \\
(83.9-92 \cdot 5)\end{array}$ & $\begin{array}{l}28 \cdot 0 \\
(19 \cdot 7-36 \cdot 2)\end{array}$ & $\begin{array}{l}46 \cdot 38 \\
(38 \cdot 3-54 \cdot 3)\end{array}$ & $\begin{array}{l}61.9 \\
(55 \cdot 8-68 \cdot 0)\end{array}$ & .. &. &.. \\
\hline \multicolumn{11}{|l|}{ Netherlands* } \\
\hline 2000-04 & $\begin{array}{l}83 \cdot 9 \\
(83 \cdot 4-84 \cdot 4)\end{array}$ & $\begin{array}{l}66 \cdot 1 \\
(64 \cdot 1-68 \cdot 0)\end{array}$ & $\begin{array}{l}36 \cdot 3 \\
(35 \cdot 1-37 \cdot 6)\end{array}$ & $\begin{array}{l}83 \cdot 4 \\
(82 \cdot 7-84 \cdot 1)\end{array}$ & $\begin{array}{l}21 \cdot 4 \\
(20 \cdot 4-22 \cdot 5)\end{array}$ & $\begin{array}{l}39 \cdot 4 \\
(38 \cdot 2-40 \cdot 6)\end{array}$ & $\begin{array}{l}54 \cdot 7 \\
(53 \cdot 9-55 \cdot 4)\end{array}$ & $\begin{array}{l}62 \cdot 7 \\
(58 \cdot 4-67 \cdot 0)\end{array}$ & $\begin{array}{l}84 \cdot 1 \\
(80 \cdot 7-87 \cdot 4)\end{array}$ & $\begin{array}{c}86 \cdot 5 \\
(81 \cdot 9-91 \cdot 2)\end{array}$ \\
\hline 2005-09 & $\begin{array}{l}85 \cdot 8 \\
(85 \cdot 3-86 \cdot 3)\end{array}$ & $\begin{array}{l}65 \cdot 5 \\
(63 \cdot 6-67 \cdot 3)\end{array}$ & $\begin{array}{l}37 \cdot 2 \\
(36 \cdot 0-38 \cdot 5)\end{array}$ & $\begin{array}{l}87.5 \\
(86.9-88.1)\end{array}$ & $\begin{array}{l}26 \cdot 3 \\
(25 \cdot 2-27 \cdot 3)\end{array}$ & $\begin{array}{l}49 \cdot 9 \\
(48 \cdot 8-51 \cdot 0)\end{array}$ & $\begin{array}{l}63 \cdot 7 \\
(63 \cdot 0-64 \cdot 4)\end{array}$ & $\begin{array}{l}66 \cdot 0 \\
(61 \cdot 9-70 \cdot 1)\end{array}$ & $\begin{array}{l}89 \cdot 8 \\
(86 \cdot 9-92 \cdot 6)\end{array}$ & $\begin{array}{c}86 \cdot 5 \\
(82 \cdot 3-90 \cdot 8)\end{array}$ \\
\hline 2010-14 & $\begin{array}{l}86 \cdot 6 \\
(86 \cdot 1-87 \cdot 1)\end{array}$ & $\begin{array}{l}67 \cdot 5 \\
(65 \cdot 6-69 \cdot 3)\end{array}$ & $\begin{array}{l}37 \cdot 5 \\
(36 \cdot 2-38 \cdot 7)\end{array}$ & $\begin{array}{l}88.5 \\
(87.9-89.0)\end{array}$ & $\begin{array}{l}28 \cdot 2 \\
(27 \cdot 2-29 \cdot 3)\end{array}$ & $\begin{array}{l}52 \cdot 2 \\
(51 \cdot 2-53 \cdot 2)\end{array}$ & $\begin{array}{l}66 \cdot 4 \\
(65 \cdot 8-67 \cdot 1)\end{array}$ & $\begin{array}{l}69 \cdot 1 \\
(65 \cdot 1-73 \cdot 1)\end{array}$ & $\begin{array}{l}90 \cdot 4 \\
(87 \cdot 5-93 \cdot 3)\end{array}$ & $\begin{array}{c}87.9 \\
(83 \cdot 5-92 \cdot 2)\end{array}$ \\
\hline
\end{tabular}




\begin{tabular}{|c|c|c|c|c|c|c|c|c|c|c|}
\hline & \multicolumn{3}{|c|}{ Women's cancers } & \multirow[t]{2}{*}{ Prostate } & \multirow[t]{2}{*}{$\begin{array}{l}\text { Brain } \\
\text { (adults) }\end{array}$} & \multicolumn{2}{|c|}{$\begin{array}{l}\text { Haemopoietic malignancies } \\
\text { (adults) }\end{array}$} & \multicolumn{3}{|c|}{ Childhood malignancies } \\
\hline & Breast & Cervix & Ovary & & & Myeloid & Lymphoid & Brain & $\begin{array}{l}\text { Acute } \\
\text { lymphoblastic } \\
\text { leukaemia }\end{array}$ & Lymphoma \\
\hline \multicolumn{11}{|c|}{ (Continued from previous page) } \\
\hline \multicolumn{11}{|l|}{ Norway* } \\
\hline 2000-04 & $\begin{array}{l}84 \cdot 7 \\
(83 \cdot 7-85 \cdot 8)\end{array}$ & $\begin{array}{l}70 \cdot 9 \\
(68 \cdot 0-73 \cdot 7)\end{array}$ & $\begin{array}{l}41 \cdot 4 \\
(39 \cdot 3-43 \cdot 6)\end{array}$ & $\begin{array}{l}83 \cdot 8 \\
(82 \cdot 8-84 \cdot 9)\end{array}$ & $\begin{array}{l}32 \cdot 4 \\
(30 \cdot 5-34 \cdot 3)\end{array}$ & $\begin{array}{l}41 \cdot 5 \\
(39 \cdot 2-43 \cdot 8)\end{array}$ & $\begin{array}{l}57 \cdot 5 \\
(56 \cdot 1-58 \cdot 8)\end{array}$ & $\begin{array}{l}79 \cdot 7 \\
(73 \cdot 8-85 \cdot 5)\end{array}$ & $\begin{array}{l}87 \cdot 7 \\
(82 \cdot 4-93 \cdot 1)\end{array}$ & $\begin{array}{c}93 \cdot 3 \\
(87 \cdot 2-99 \cdot 4)\end{array}$ \\
\hline 2005-09 & $\begin{array}{l}87 \cdot 2 \\
(86 \cdot 1-88 \cdot 3)\end{array}$ & $\begin{array}{l}70 \cdot 7 \\
(67 \cdot 8-73 \cdot 6)\end{array}$ & $\begin{array}{l}42 \cdot 8 \\
(40 \cdot 7-45 \cdot 0)\end{array}$ & $\begin{array}{l}90 \cdot 3 \\
(89 \cdot 5-91 \cdot 1)\end{array}$ & $\begin{array}{l}36 \cdot 7 \\
(34 \cdot 9-38 \cdot 5)\end{array}$ & $\begin{array}{l}50 \cdot 9 \\
(48 \cdot 8-53 \cdot 0)\end{array}$ & $\begin{array}{l}64 \cdot 9 \\
(63 \cdot 7-66 \cdot 2)\end{array}$ & $\begin{array}{l}75 \cdot 9 \\
(69 \cdot 7-82 \cdot 1)\end{array}$ & $\begin{array}{l}84.9 \\
(78 \cdot 2-91.6)\end{array}$ & $\begin{array}{c}90 \cdot 9 \\
(84 \cdot 3-97 \cdot 5)\end{array}$ \\
\hline 2010-14 & $\begin{array}{l}87 \cdot 7 \\
(86 \cdot 6-88 \cdot 8)\end{array}$ & $\begin{array}{l}73 \cdot 3 \\
(70 \cdot 3-76 \cdot 3)\end{array}$ & $\begin{array}{l}45 \cdot 5 \\
(43 \cdot 3-47 \cdot 7)\end{array}$ & $\begin{array}{l}92 \cdot 9 \\
(92 \cdot 2-93 \cdot 7)\end{array}$ & $\begin{array}{l}36 \cdot 8 \\
(34 \cdot 9-38 \cdot 7)\end{array}$ & $\begin{array}{l}52 \cdot 7 \\
(50 \cdot 7-54 \cdot 7)\end{array}$ & $\begin{array}{l}68 \cdot 4 \\
(67 \cdot 2-69 \cdot 6)\end{array}$ & $\begin{array}{l}74 \cdot 3 \\
(67 \cdot 6-81 \cdot 0)\end{array}$ & $\begin{array}{l}83 \cdot 0 \\
(76 \cdot 5-89 \cdot 5)\end{array}$ & $\begin{array}{c}95 \cdot 2 \\
(90 \cdot 9-99 \cdot 4)\end{array}$ \\
\hline \multicolumn{11}{|c|}{ Poland (16 registries)* } \\
\hline $2000-04$ & $\begin{array}{l}71 \cdot 3 \\
(70 \cdot 7-71 \cdot 9)\end{array}$ & $\begin{array}{l}51 \cdot 6 \\
(50 \cdot 8-52 \cdot 5)\end{array}$ & $\begin{array}{l}32 \cdot 7 \\
(31 \cdot 9-33 \cdot 6)\end{array}$ & $\begin{array}{l}68 \cdot 8 \\
(67 \cdot 9-69 \cdot 7)\end{array}$ & $\begin{array}{l}26 \cdot 6 \\
(25 \cdot 6-27 \cdot 7)\end{array}$ & $\begin{array}{l}18 \cdot 9 \\
(17 \cdot 9-19 \cdot 9)\end{array}$ & $\begin{array}{l}40 \cdot 3 \\
(39 \cdot 6-41 \cdot 0)\end{array}$ & $\begin{array}{l}62 \cdot 6 \\
(59 \cdot 2-66 \cdot 0)\end{array}$ & $\begin{array}{l}79.6 \\
(76 \cdot 3-82 \cdot 9)\end{array}$ & $\begin{array}{l}81 \cdot 7 \\
(77 \cdot 8-85 \cdot 6)\end{array}$ \\
\hline 2005-09 & $\begin{array}{l}74 \cdot 7 \\
(74 \cdot 2-75 \cdot 2)\end{array}$ & $\begin{array}{l}54 \cdot 4 \\
(53 \cdot 6-55 \cdot 3)\end{array}$ & $\begin{array}{l}35 \cdot 4 \\
(34 \cdot 6-36 \cdot 2)\end{array}$ & $\begin{array}{l}75 \cdot 0 \\
(74 \cdot 4-75 \cdot 7)\end{array}$ & $\begin{array}{l}29 \cdot 0 \\
(28 \cdot 2-29 \cdot 8)\end{array}$ & $\begin{array}{l}26 \cdot 3 \\
(25 \cdot 2-27 \cdot 5)\end{array}$ & $\begin{array}{l}47 \cdot 8 \\
(47 \cdot 2-48 \cdot 5)\end{array}$ & $\begin{array}{l}61 \cdot 7 \\
(58 \cdot 1-65 \cdot 3)\end{array}$ & $\begin{array}{l}84 \cdot 4 \\
(81 \cdot 7-87 \cdot 0)\end{array}$ & $\begin{array}{c}89 \cdot 4 \\
(86 \cdot 4-92 \cdot 4)\end{array}$ \\
\hline 2010-14 & $\begin{array}{l}76 \cdot 5 \\
(76 \cdot 1-77 \cdot 0)\end{array}$ & $\begin{array}{l}55 \cdot 1 \\
(54 \cdot 2-55 \cdot 9)\end{array}$ & $\begin{array}{l}37 \cdot 5 \\
(36 \cdot 7-38 \cdot 3)\end{array}$ & $\begin{array}{l}78 \cdot 1 \\
(77 \cdot 5-78 \cdot 7)\end{array}$ & $\begin{array}{l}28 \cdot 2 \\
(27 \cdot 4-28 \cdot 9)\end{array}$ & $\begin{array}{l}27 \cdot 3 \\
(26 \cdot 2-28 \cdot 4)\end{array}$ & $\begin{array}{l}52 \cdot 1 \\
(51 \cdot 4-52 \cdot 8)\end{array}$ & $\begin{array}{l}62 \cdot 5 \\
(58 \cdot 7-66 \cdot 4)\end{array}$ & $\begin{array}{l}86 \cdot 9 \\
(84 \cdot 1-89 \cdot 7)\end{array}$ & $\begin{array}{c}92 \cdot 6 \\
(89 \cdot 7-95 \cdot 5)\end{array}$ \\
\hline \multicolumn{11}{|c|}{ Portugal (four registries)* } \\
\hline $2000-04$ & $\begin{array}{l}81 \cdot 6 \\
(80 \cdot 7-82 \cdot 5)\end{array}$ & $\begin{array}{l}60 \cdot 4 \\
(58 \cdot 6-62 \cdot 2)\end{array}$ & $\begin{array}{l}39 \cdot 9 \\
(37 \cdot 7-42 \cdot 1)\end{array}$ & $\begin{array}{l}87 \cdot 2 \\
(86 \cdot 2-88 \cdot 1)\end{array}$ & $\begin{array}{l}22 \cdot 9 \\
(21 \cdot 3-24 \cdot 6)\end{array}$ & $\begin{array}{l}43 \cdot 7 \\
(41 \cdot 6-45 \cdot 8)\end{array}$ & $\begin{array}{l}51 \cdot 3 \\
(50 \cdot 1-52 \cdot 6)\end{array}$ & $\begin{array}{l}62 \cdot 0 \\
(55 \cdot 0-69 \cdot 1)\end{array}$ & $\begin{array}{l}80 \cdot 8 \\
(75 \cdot 3-86 \cdot 4)\end{array}$ & $\begin{array}{l}86 \cdot 2 \\
(81 \cdot 0-91 \cdot 4)\end{array}$ \\
\hline 2005-09 & $\begin{array}{l}86 \cdot 1 \\
(85 \cdot 3-86 \cdot 9)\end{array}$ & $\begin{array}{l}65 \cdot 3 \\
(63 \cdot 6-67 \cdot 0)\end{array}$ & $\begin{array}{l}41 \cdot 8 \\
(39 \cdot 7-44 \cdot 0)\end{array}$ & $\begin{array}{l}90 \cdot 0 \\
(89 \cdot 3-90 \cdot 8)\end{array}$ & $\begin{array}{l}23 \cdot 8 \\
(22 \cdot 4-25 \cdot 3)\end{array}$ & $\begin{array}{l}49 \cdot 0 \\
(47 \cdot 1-50 \cdot 9)\end{array}$ & $\begin{array}{l}58 \cdot 2 \\
(57 \cdot 1-59 \cdot 2)\end{array}$ & $\begin{array}{l}63 \cdot 1 \\
(56 \cdot 5-69 \cdot 7)\end{array}$ & $\begin{array}{l}84 \cdot 1 \\
(78 \cdot 8-89 \cdot 5)\end{array}$ & $\begin{array}{l}94 \cdot 0 \\
(90 \cdot 1-97 \cdot 9)\end{array}$ \\
\hline 2010-14 & $\begin{array}{l}87 \cdot 6 \\
(85 \cdot 9-89 \cdot 3)\end{array}$ & $\begin{array}{l}66 \cdot 2 \\
(62 \cdot 6-69 \cdot 8)\end{array}$ & $\begin{array}{l}43 \cdot 6 \\
(38 \cdot 7-48 \cdot 4)\end{array}$ & $\begin{array}{l}90 \cdot 9 \\
(89 \cdot 1-92 \cdot 6)\end{array}$ & $\begin{array}{l}22 \cdot 7 \\
(19 \cdot 7-25 \cdot 8)\end{array}$ & $\begin{array}{l}49 \cdot 8 \\
(45 \cdot 4-54 \cdot 2)\end{array}$ & $\begin{array}{l}59 \cdot 7 \\
(57 \cdot 3-62 \cdot 1)\end{array}$ & $\begin{array}{l}70 \cdot 5 \\
(57 \cdot 0-84 \cdot 1)\end{array}$ & $\begin{array}{l}89.8 \\
(80 \cdot 7-98 \cdot 9)\end{array}$ & $\begin{array}{l}94 \cdot 7 \\
(88 \cdot 9-100 \cdot 0)\end{array}$ \\
\hline \multicolumn{11}{|c|}{ Romania (Cluj) } \\
\hline $2000-04$ &.$\cdot$ & .. &.$\cdot$ &.$\cdot$ &.. &.$\cdot$ & .. &.. & .. & .. \\
\hline 2005-09 & $\begin{array}{l}74 \cdot 8 \\
(70 \cdot 4-79 \cdot 3)\end{array}$ & $\begin{array}{l}61 \cdot 7 \\
(57 \cdot 0-66 \cdot 5)\end{array}$ & $\begin{array}{l}28 \cdot 95 \\
(22 \cdot 3-35 \cdot 6)\end{array}$ & $\begin{array}{l}78 \cdot 2 \\
(72 \cdot 5-83 \cdot 8)\end{array}$ & $\begin{array}{l}20 \cdot 45 \\
(14 \cdot 0-26 \cdot 7)\end{array}$ & $\begin{array}{l}44 \cdot 9 \\
(37 \cdot 2-52 \cdot 5)\end{array}$ & $\begin{array}{l}47 \cdot 3 \\
(42 \cdot 0-52 \cdot 5)\end{array}$ & .. & .. & .. \\
\hline 2010-14 & $\begin{array}{l}74 \cdot 8 \\
(68 \cdot 5-81 \cdot 1)\end{array}$ & $\begin{array}{l}65 \cdot 3 \\
(59 \cdot 7-70 \cdot 9)\end{array}$ & $\begin{array}{l}37 \cdot 25 \\
(29 \cdot 7-44 \cdot 6)\end{array}$ & $\begin{array}{l}77 \cdot 1 \\
(70 \cdot 0-84 \cdot 2)\end{array}$ & $\begin{array}{l}34 \cdot 05 \\
(23 \cdot 8-44 \cdot 1)\end{array}$ & $\begin{array}{l}51 \cdot 5 \\
(42 \cdot 7-60 \cdot 3)\end{array}$ & $\begin{array}{l}40 \cdot 4 \\
(33 \cdot 3-47 \cdot 5)\end{array}$ & $\begin{array}{l}60 \cdot 1 \dagger \\
(31 \cdot 6-88 \cdot 5)\end{array}$ & $\begin{array}{l}53 \cdot 9 \dagger \\
(28 \cdot 2-79 \cdot 6)\end{array}$ & .. \\
\hline \multicolumn{11}{|c|}{ Russia (five registries) } \\
\hline $2000-04$ & $\begin{array}{l}71 \cdot 6 \\
(69 \cdot 8-73 \cdot 4)\end{array}$ & $\begin{array}{l}59 \cdot 8 \\
(57 \cdot 3-62 \cdot 2)\end{array}$ & $\begin{array}{l}38 \cdot 1 \\
(35 \cdot 2-41 \cdot 1)\end{array}$ & $\begin{array}{l}58 \cdot 8 \\
(55 \cdot 4-62 \cdot 1)\end{array}$ & $\begin{array}{l}25 \cdot 2 \\
(21 \cdot 3-29 \cdot 0)\end{array}$ & $\begin{array}{l}33 \cdot 1 \\
(28 \cdot 7-37 \cdot 5)\end{array}$ & $\begin{array}{l}40 \cdot 5 \\
(37 \cdot 4-43 \cdot 5)\end{array}$ & $\begin{array}{l}61 \cdot 2 \\
(50 \cdot 4-72 \cdot 0)\end{array}$ & $\begin{array}{l}71 \cdot 3 \\
(62 \cdot 6-80 \cdot 0)\end{array}$ & $\begin{array}{c}67 \cdot 0 \\
(54 \cdot 9-79 \cdot 1)\end{array}$ \\
\hline $2005-09$ & $\begin{array}{l}67 \cdot 7 \\
(66 \cdot 4-69 \cdot 0)\end{array}$ & $\begin{array}{l}58 \cdot 0 \\
(56 \cdot 0-59 \cdot 9)\end{array}$ & $\begin{array}{l}33 \cdot 2 \\
(31 \cdot 3-35 \cdot 0)\end{array}$ & $\begin{array}{l}68 \cdot 6 \\
(66 \cdot 2-71 \cdot 0)\end{array}$ & $\begin{array}{l}21 \cdot 2 \\
(19 \cdot 2-23 \cdot 2)\end{array}$ & $\begin{array}{l}35 \cdot 3 \\
(31 \cdot 7-39 \cdot 0)\end{array}$ & $\begin{array}{l}42 \cdot 4 \\
(39 \cdot 8-45 \cdot 0)\end{array}$ & $\begin{array}{l}61 \cdot 2 \\
(50 \cdot 3-72 \cdot 0)\end{array}$ & $\begin{array}{l}74 \cdot 2 \\
(65 \cdot 3-83 \cdot 0)\end{array}$ & $\begin{array}{c}73 \cdot 5 \\
(62 \cdot 6-84 \cdot 4)\end{array}$ \\
\hline $2010-14$ & $\begin{array}{l}70 \cdot 8 \\
(69 \cdot 5-72 \cdot 1)\end{array}$ & $\begin{array}{l}57 \cdot 7 \\
(55 \cdot 7-59 \cdot 7)\end{array}$ & $\begin{array}{l}34 \cdot 8 \\
(32 \cdot 8-36 \cdot 8)\end{array}$ & $\begin{array}{l}79 \cdot 3 \\
(77 \cdot 1-81 \cdot 5)\end{array}$ & $\begin{array}{l}22 \cdot 8 \\
(20 \cdot 8-24 \cdot 9)\end{array}$ & $\begin{array}{l}33 \cdot 2 \\
(29 \cdot 8-36 \cdot 7)\end{array}$ & $\begin{array}{l}45 \cdot 5 \\
(42 \cdot 9-48 \cdot 2)\end{array}$ & $\begin{array}{l}61 \cdot 7 \\
(51 \cdot 5-71 \cdot 8)\end{array}$ & $\begin{array}{l}76 \cdot 9 \\
(68 \cdot 4-85 \cdot 4)\end{array}$ & $\begin{array}{c}92 \cdot 1 \\
(84 \cdot 6-99 \cdot 6)\end{array}$ \\
\hline \multicolumn{11}{|l|}{ Slovakia* } \\
\hline $2000-04$ & $\begin{array}{l}75 \cdot 3 \\
(73 \cdot 5-77 \cdot 2)\end{array}$ & $\begin{array}{l}61 \cdot 8 \\
(59 \cdot 4-64 \cdot 3)\end{array}$ & $\begin{array}{l}35 \cdot 0 \\
(32 \cdot 4-37 \cdot 6)\end{array}$ & $\begin{array}{l}63 \cdot 6 \\
(61 \cdot 1-66 \cdot 0)\end{array}$ & $\begin{array}{l}22 \cdot 05 \\
(20 \cdot 0-24 \cdot 0)\end{array}$ & $\begin{array}{l}35 \cdot 15 \\
(32 \cdot 2-38 \cdot 1)\end{array}$ & $\begin{array}{l}46 \cdot 6 \\
(44 \cdot 6-48 \cdot 6)\end{array}$ & $\begin{array}{l}67 \cdot 9 \\
(60 \cdot 5-75 \cdot 4)\end{array}$ & $\begin{array}{l}79 \cdot 2 \\
(71 \cdot 8-86 \cdot 5)\end{array}$ & $\begin{array}{c}83 \cdot 8 \\
(75 \cdot 1-92 \cdot 5)\end{array}$ \\
\hline 2005-09 & $\begin{array}{l}76 \cdot 6 \\
(75 \cdot 1-78 \cdot 2)\end{array}$ & $\begin{array}{l}58 \cdot 9 \\
(56 \cdot 5-61 \cdot 3)\end{array}$ & $\begin{array}{l}34 \cdot 5 \\
(31 \cdot 7-37 \cdot 3)\end{array}$ & $\begin{array}{l}74 \cdot 4 \\
(72 \cdot 7-76 \cdot 2)\end{array}$ & $\begin{array}{l}27 \cdot 2 \\
(25 \cdot 3-29 \cdot 0)\end{array}$ & $\begin{array}{l}37 \cdot 1 \\
(34 \cdot 8-39 \cdot 4)\end{array}$ & $\begin{array}{l}49 \cdot 6 \\
(48 \cdot 0-51 \cdot 2)\end{array}$ & $\begin{array}{l}70 \cdot 0 \\
(62 \cdot 5-77 \cdot 5)\end{array}$ & $\begin{array}{l}80 \cdot 7 \\
(73 \cdot 8-87 \cdot 7)\end{array}$ & $\begin{array}{l}94 \cdot 6 \\
(90 \cdot 1-99 \cdot 1)\end{array}$ \\
\hline 2010-14 & $\begin{array}{l}75 \cdot 5 \\
(72 \cdot 4-78 \cdot 5)\end{array}$ & $\begin{array}{l}60 \cdot 5 \\
(56 \cdot 2-64 \cdot 9)\end{array}$ & $\begin{array}{l}33 \cdot 4 \\
(28 \cdot 6-38 \cdot 2)\end{array}$ & $\begin{array}{l}74 \cdot 7 \\
(70 \cdot 9-78 \cdot 6)\end{array}$ & $\begin{array}{l}28 \cdot 5 \\
(24 \cdot 3-32 \cdot 8)\end{array}$ & $\begin{array}{l}37 \cdot 5 \\
(32 \cdot 2-42 \cdot 8)\end{array}$ & $\begin{array}{l}51 \cdot 6 \\
(48 \cdot 0-55 \cdot 2)\end{array}$ & $\begin{array}{l}80 \cdot 6 \\
(69 \cdot 4-91 \cdot 7)\end{array}$ & $\begin{array}{l}87 \cdot 0 \\
(77 \cdot 3-96 \cdot 7)\end{array}$ & $\begin{array}{l}88 \cdot 6 \\
(75 \cdot 4-100 \cdot 0)\end{array}$ \\
\hline \multicolumn{11}{|l|}{ Slovenia* } \\
\hline $2000-04$ & $\begin{array}{l}78 \cdot 7 \\
(76 \cdot 9-80 \cdot 5)\end{array}$ & $\begin{array}{l}67 \cdot 2 \\
(63 \cdot 8-70 \cdot 7)\end{array}$ & $\begin{array}{l}37 \cdot 8 \\
(34 \cdot 4-41 \cdot 2)\end{array}$ & $\begin{array}{l}74 \cdot 4 \\
(71 \cdot 9-76 \cdot 9)\end{array}$ & $\begin{array}{l}23 \cdot 0 \\
(19 \cdot 9-26 \cdot 1)\end{array}$ & $\begin{array}{l}42 \cdot 4 \\
(38 \cdot 5-46 \cdot 2)\end{array}$ & $\begin{array}{l}52 \cdot 7 \\
(50 \cdot 2-55 \cdot 2)\end{array}$ & $\begin{array}{l}76 \cdot 5 \\
(66 \cdot 8-86 \cdot 3)\end{array}$ & $\begin{array}{l}89 \cdot 7 \\
(82 \cdot 1-97 \cdot 3)\end{array}$ & $\begin{array}{l}80 \cdot 1 \\
(68 \cdot 7-91 \cdot 5)\end{array}$ \\
\hline 2005-09 & $\begin{array}{l}82 \cdot 5 \\
(81 \cdot 0-84 \cdot 1)\end{array}$ & $\begin{array}{l}67 \cdot 1 \\
(63 \cdot 3-70 \cdot 9)\end{array}$ & $\begin{array}{l}35 \cdot 4 \\
(32 \cdot 3-38 \cdot 4)\end{array}$ & $\begin{array}{l}83 \cdot 2 \\
(81 \cdot 6-84 \cdot 8)\end{array}$ & $\begin{array}{l}24 \cdot 1 \\
(21 \cdot 2-27 \cdot 0)\end{array}$ & $\begin{array}{l}39 \cdot 8 \\
(36 \cdot 6-42 \cdot 9)\end{array}$ & $\begin{array}{l}56 \cdot 7 \\
(54 \cdot 6-58 \cdot 9)\end{array}$ & $\begin{array}{l}61 \cdot 2 \\
(49 \cdot 2-73 \cdot 1)\end{array}$ & $\begin{array}{l}79 \cdot 1 \dagger \\
(67 \cdot 1-91 \cdot 1)\end{array}$ & $\begin{array}{l}100 \cdot 0 \dagger \\
(86 \cdot 3-100 \cdot 0)\end{array}$ \\
\hline \multirow[t]{2}{*}{ 2010-14 } & $\begin{array}{l}83 \cdot 5 \\
(81 \cdot 8-85 \cdot 2)\end{array}$ & $\begin{array}{l}65 \cdot 5 \\
(61 \cdot 3-69 \cdot 8)\end{array}$ & $\begin{array}{l}37 \cdot 0 \\
(33 \cdot 4-40 \cdot 5)\end{array}$ & $\begin{array}{l}85 \cdot 0 \\
(83 \cdot 3-86 \cdot 7)\end{array}$ & $\begin{array}{l}24 \cdot 8 \\
(21 \cdot 4-28 \cdot 3)\end{array}$ & $\begin{array}{l}37 \cdot 5 \\
(34 \cdot 2-40 \cdot 7)\end{array}$ & $\begin{array}{l}59 \cdot 0 \\
(56 \cdot 6-61 \cdot 3)\end{array}$ & $\begin{array}{l}60 \cdot 1 \\
(44 \cdot 2-76 \cdot 1)\end{array}$ & $\begin{array}{l}70 \cdot 1 \\
(54 \cdot 4-85 \cdot 8)\end{array}$ & $\begin{array}{l}100 \cdot 0 \\
(100 \cdot 0-100 \cdot 0)\end{array}$ \\
\hline & & & & & & & & & \multicolumn{2}{|c|}{ (Table 7 continues on next page } \\
\hline
\end{tabular}




\begin{tabular}{|c|c|c|c|c|c|c|c|c|c|c|}
\hline & \multicolumn{3}{|c|}{ Women's cancers } & \multirow[t]{2}{*}{ Prostate } & \multirow[t]{2}{*}{$\begin{array}{l}\text { Brain } \\
\text { (adults) }\end{array}$} & \multicolumn{2}{|c|}{$\begin{array}{l}\text { Haemopoietic malignancies } \\
\text { (adults) }\end{array}$} & \multicolumn{3}{|c|}{ Childhood malignancies } \\
\hline & Breast & Cervix & Ovary & & & Myeloid & Lymphoid & Brain & $\begin{array}{l}\text { Acute } \\
\text { lymphoblastic } \\
\text { leukaemia }\end{array}$ & Lymphoma \\
\hline \multicolumn{11}{|c|}{ (Continued from previous page) } \\
\hline \multicolumn{11}{|c|}{ Spain (ten registries) } \\
\hline $2000-04$ & $\begin{array}{l}82 \cdot 9 \\
(82 \cdot 0-83 \cdot 8)\end{array}$ & $\begin{array}{l}63 \cdot 6 \\
(61 \cdot 3-65 \cdot 9)\end{array}$ & $\begin{array}{l}36 \cdot 0 \\
(34 \cdot 1-37 \cdot 9)\end{array}$ & $\begin{array}{l}85 \cdot 0 \\
(84 \cdot 1-85 \cdot 9)\end{array}$ & $\begin{array}{l}21 \cdot 6 \\
(20 \cdot 2-23 \cdot 0)\end{array}$ & $\begin{array}{l}45 \cdot 4 \\
(43 \cdot 6-47 \cdot 2)\end{array}$ & $\begin{array}{l}58 \cdot 2 \\
(57 \cdot 1-59 \cdot 3)\end{array}$ & $\begin{array}{l}63 \cdot 6 \\
(57 \cdot 7-69 \cdot 4)\end{array}$ & $\begin{array}{l}80 \cdot 9 \\
(76 \cdot 3-85 \cdot 5)\end{array}$ & $\begin{array}{l}85 \cdot 8 \\
(80 \cdot 2-91 \cdot 4)\end{array}$ \\
\hline 2005-09 & $\begin{array}{l}84 \cdot 6 \\
(83 \cdot 8-85 \cdot 4)\end{array}$ & $\begin{array}{l}64 \cdot 5 \\
(62 \cdot 2-66 \cdot 8)\end{array}$ & $\begin{array}{l}37 \cdot 9 \\
(36 \cdot 1-39 \cdot 6)\end{array}$ & $\begin{array}{l}90 \cdot 4 \\
(89 \cdot 7-91 \cdot 1)\end{array}$ & $\begin{array}{l}27 \cdot 2 \\
(25 \cdot 8-28 \cdot 7)\end{array}$ & $\begin{array}{l}52 \cdot 3 \\
(50 \cdot 8-53 \cdot 8)\end{array}$ & $\begin{array}{l}62 \cdot 2 \\
(61 \cdot 3-63 \cdot 2)\end{array}$ & $\begin{array}{l}63 \cdot 4 \\
(58 \cdot 2-68 \cdot 6)\end{array}$ & $\begin{array}{l}80 \cdot 0 \\
(75 \cdot 4-84 \cdot 5)\end{array}$ & $\begin{array}{l}89.9 \\
(85 \cdot 9-94 \cdot 0)\end{array}$ \\
\hline 2010-14 & $\begin{array}{l}85 \cdot 2 \\
(84 \cdot 0-86 \cdot 5)\end{array}$ & $\begin{array}{l}64 \cdot 5 \\
(60 \cdot 8-68 \cdot 1)\end{array}$ & $\begin{array}{l}39 \cdot 8 \\
(36 \cdot 9-42 \cdot 7)\end{array}$ & $\begin{array}{l}89 \cdot 7 \\
(88 \cdot 6-90 \cdot 7)\end{array}$ & $\begin{array}{l}27 \cdot 4 \\
(25 \cdot 2-29 \cdot 7)\end{array}$ & $\begin{array}{l}50 \cdot 0 \\
(47 \cdot 7-52 \cdot 3)\end{array}$ & $\begin{array}{l}62 \cdot 0 \\
(60 \cdot 5-63 \cdot 6)\end{array}$ & $\begin{array}{l}66 \cdot 2 \\
(58 \cdot 0-74 \cdot 4)\end{array}$ & $\begin{array}{l}84 \cdot 7 \\
(77 \cdot 6-91 \cdot 7)\end{array}$ & $\begin{array}{l}92 \cdot 9 \\
(87 \cdot 5-98 \cdot 3)\end{array}$ \\
\hline \multicolumn{11}{|l|}{ Sweden* } \\
\hline 2000-04 & $\begin{array}{l}85 \cdot 6 \\
(84 \cdot 9-86 \cdot 3)\end{array}$ & $\begin{array}{l}66 \cdot 9 \\
(64 \cdot 7-69 \cdot 1)\end{array}$ & $\begin{array}{l}43 \cdot 2 \\
(41 \cdot 6-44 \cdot 8)\end{array}$ & $\begin{array}{l}85 \cdot 9 \\
(85 \cdot 3-86 \cdot 5)\end{array}$ & $\begin{array}{l}26 \cdot 5 \\
(25 \cdot 1-28 \cdot 0)\end{array}$ & $\begin{array}{l}30 \cdot 7 \\
(28 \cdot 9-32 \cdot 6)\end{array}$ & $\begin{array}{l}58 \cdot 5 \\
(57 \cdot 6-59 \cdot 5)\end{array}$ & $\begin{array}{l}75 \cdot 9 \\
(70 \cdot 5-81 \cdot 3)\end{array}$ & $\begin{array}{l}86 \cdot 8 \\
(82.6-90 \cdot 9)\end{array}$ & $\begin{array}{l}84 \cdot 7 \\
(75 \cdot 8-93 \cdot 6)\end{array}$ \\
\hline 2005-09 & $\begin{array}{l}87 \cdot 9 \\
(87 \cdot 2-88 \cdot 5)\end{array}$ & $\begin{array}{l}67 \cdot 7 \\
(65 \cdot 6-69 \cdot 9)\end{array}$ & $\begin{array}{l}42 \cdot 9 \\
(41 \cdot 2-44 \cdot 6)\end{array}$ & $\begin{array}{l}90 \cdot 1 \\
(89 \cdot 6-90 \cdot 6)\end{array}$ & $\begin{array}{l}29 \cdot 0 \\
(27 \cdot 5-30 \cdot 4)\end{array}$ & $\begin{array}{l}54 \cdot 6 \\
(53 \cdot 1-56 \cdot 1)\end{array}$ & $\begin{array}{l}64 \cdot 2 \\
(63 \cdot 3-65 \cdot 1)\end{array}$ & $\begin{array}{l}78 \cdot 4 \\
(73 \cdot 2-83 \cdot 6)\end{array}$ & $\begin{array}{l}87.1 \\
(82.6-91 \cdot 5)\end{array}$ & $\begin{array}{l}88 \cdot 6 \\
(81 \cdot 5-95 \cdot 8)\end{array}$ \\
\hline $2010-14$ & $\begin{array}{l}88 \cdot 8 \\
(88 \cdot 2-89 \cdot 4)\end{array}$ & $\begin{array}{l}68 \cdot 3 \\
(66 \cdot 1-70 \cdot 4)\end{array}$ & $\begin{array}{l}46 \cdot 5 \\
(44 \cdot 8-48 \cdot 2)\end{array}$ & $\begin{array}{l}90 \cdot 7 \\
(90 \cdot 2-91 \cdot 2)\end{array}$ & $\begin{array}{l}31 \cdot 6 \\
(30 \cdot 1-33 \cdot 0)\end{array}$ & $\begin{array}{l}57 \cdot 5 \\
(56 \cdot 0-58 \cdot 9)\end{array}$ & $\begin{array}{l}66 \cdot 7 \\
(65 \cdot 9-67 \cdot 6)\end{array}$ & $\begin{array}{l}79 \cdot 8 \\
(74 \cdot 9-84 \cdot 7)\end{array}$ & $\begin{array}{l}89 \cdot 0 \\
(84 \cdot 6-93 \cdot 3)\end{array}$ & $\begin{array}{c}88.0 \\
(80 \cdot 4-95 \cdot 7)\end{array}$ \\
\hline \multicolumn{11}{|c|}{ Switzerland (ten registries) $\ddagger$} \\
\hline 2000-04 & $\begin{array}{l}84 \cdot 4 \\
(83 \cdot 3-85 \cdot 5)\end{array}$ & $\begin{array}{l}63 \cdot 4 \\
(58 \cdot 4-68 \cdot 4)\end{array}$ & $\begin{array}{l}36 \cdot 9 \\
(34 \cdot 4-39 \cdot 5)\end{array}$ & $\begin{array}{l}86 \cdot 9 \\
(85 \cdot 8-87 \cdot 9)\end{array}$ & $\begin{array}{l}26 \cdot 4 \\
(23 \cdot 9-28 \cdot 8)\end{array}$ & $\begin{array}{l}46 \cdot 5 \\
(43 \cdot 9-49 \cdot 1)\end{array}$ & $\begin{array}{l}61 \cdot 6 \\
(60 \cdot 1-63 \cdot 2)\end{array}$ & $\begin{array}{l}73 \cdot 7 \\
(67 \cdot 7-79 \cdot 8)\end{array}$ & $\begin{array}{l}87 \cdot 3 \\
(82 \cdot 4-92 \cdot 2)\end{array}$ & $\begin{array}{l}94 \cdot 0 \\
(89 \cdot 5-98 \cdot 6)\end{array}$ \\
\hline 2005-09 & $\begin{array}{l}86 \cdot 4 \\
(85 \cdot 3-87 \cdot 4)\end{array}$ & $\begin{array}{l}69 \cdot 4 \\
(65 \cdot 1-73 \cdot 6)\end{array}$ & $\begin{array}{l}42 \cdot 0 \\
(39 \cdot 5-44 \cdot 4)\end{array}$ & $\begin{array}{l}88 \cdot 6 \\
(87 \cdot 6-89 \cdot 5)\end{array}$ & $\begin{array}{l}29 \cdot 0 \\
(26 \cdot 7-31 \cdot 2)\end{array}$ & $\begin{array}{l}51 \cdot 6 \\
(49 \cdot 3-53 \cdot 9)\end{array}$ & $\begin{array}{l}70 \cdot 9 \\
(69 \cdot 5-72 \cdot 3)\end{array}$ & $\begin{array}{l}68 \cdot 0 \\
(61 \cdot 2-74 \cdot 8)\end{array}$ & $\begin{array}{l}89 \cdot 2 \\
(84 \cdot 9-93 \cdot 4)\end{array}$ & $\begin{array}{l}94 \cdot 2 \\
(89 \cdot 5-98 \cdot 9)\end{array}$ \\
\hline 2010-14 & $\begin{array}{l}86 \cdot 2 \\
(85 \cdot 1-87 \cdot 3)\end{array}$ & $\begin{array}{l}71 \cdot 4 \\
(66 \cdot 6-76 \cdot 2)\end{array}$ & $\begin{array}{l}44 \cdot 1 \\
(41 \cdot 3-46 \cdot 8)\end{array}$ & $\begin{array}{l}89 \cdot 2 \\
(88 \cdot 2-90 \cdot 3)\end{array}$ & $\begin{array}{l}29 \cdot 7 \\
(27 \cdot 5-31 \cdot 9)\end{array}$ & $\begin{array}{l}49 \cdot 7 \\
(47 \cdot 2-52 \cdot 2)\end{array}$ & $\begin{array}{l}72 \cdot 0 \\
(70 \cdot 4-73 \cdot 5)\end{array}$ & $\begin{array}{l}71 \cdot 6 \\
(65 \cdot 1-78 \cdot 0)\end{array}$ & $\begin{array}{l}90 \cdot 3 \\
(86 \cdot 1-94 \cdot 5)\end{array}$ & $\begin{array}{l}93.6 \\
(88 \cdot 1-99 \cdot 1)\end{array}$ \\
\hline \multicolumn{11}{|c|}{ UK (four registries)* } \\
\hline $2000-04$ & $\begin{array}{l}79 \cdot 8 \\
(79 \cdot 5-80 \cdot 1)\end{array}$ & $\begin{array}{l}58.9 \\
(58 \cdot 0-59 \cdot 9)\end{array}$ & $\begin{array}{l}30 \cdot 2 \\
(29 \cdot 7-30 \cdot 7)\end{array}$ & $\begin{array}{l}81 \cdot 9 \\
(81 \cdot 6-82 \cdot 3)\end{array}$ & $\begin{array}{l}20 \cdot 6 \\
(20 \cdot 1-21 \cdot 2)\end{array}$ & $\begin{array}{l}42 \cdot 3 \\
(41 \cdot 7-42 \cdot 9)\end{array}$ & $\begin{array}{l}54 \cdot 3 \\
(53 \cdot 9-54 \cdot 6)\end{array}$ & $\begin{array}{l}68 \cdot 4 \\
(66 \cdot 2-70 \cdot 7)\end{array}$ & $\begin{array}{l}85 \cdot 9 \\
(84.1-87.7)\end{array}$ & $\begin{array}{c}86 \cdot 8 \\
(84 \cdot 1-89 \cdot 5)\end{array}$ \\
\hline 2005-09 & $\begin{array}{l}83 \cdot 8 \\
(83 \cdot 6-84 \cdot 1)\end{array}$ & $\begin{array}{l}61 \cdot 9 \\
(61 \cdot 0-62 \cdot 9)\end{array}$ & $\begin{array}{l}33 \cdot 2 \\
(32 \cdot 6-33 \cdot 7)\end{array}$ & $\begin{array}{l}86 \cdot 7 \\
(86 \cdot 4-86.9)\end{array}$ & $\begin{array}{l}23 \cdot 8 \\
(23 \cdot 3-24 \cdot 4)\end{array}$ & $\begin{array}{l}47 \cdot 7 \\
(47 \cdot 1-48 \cdot 2)\end{array}$ & $\begin{array}{l}60 \cdot 7 \\
(60 \cdot 4-61 \cdot 1)\end{array}$ & $\begin{array}{l}69 \cdot 1 \\
(66 \cdot 9-71 \cdot 3)\end{array}$ & $\begin{array}{l}91 \cdot 4 \\
(89.9-92 \cdot 8)\end{array}$ & $\begin{array}{l}90 \cdot 6 \\
(88 \cdot 3-93 \cdot 0)\end{array}$ \\
\hline 2010-14 & $\begin{array}{l}85 \cdot 6 \\
(85 \cdot 4-85 \cdot 9)\end{array}$ & $\begin{array}{l}63 \cdot 8 \\
(62 \cdot 8-64 \cdot 7)\end{array}$ & $\begin{array}{l}36 \cdot 2 \\
(35 \cdot 7-36 \cdot 8)\end{array}$ & $\begin{array}{l}88 \cdot 7 \\
(88.5-89.0)\end{array}$ & $\begin{array}{l}26 \cdot 3 \\
(25 \cdot 7-26 \cdot 8)\end{array}$ & $\begin{array}{l}48 \cdot 7 \\
(48 \cdot 1-49 \cdot 2)\end{array}$ & $\begin{array}{l}64 \cdot 9 \\
(64 \cdot 6-65 \cdot 3)\end{array}$ & $\begin{array}{l}71 \cdot 9 \\
(69 \cdot 8-74 \cdot 0)\end{array}$ & $\begin{array}{l}92 \cdot 2 \\
(90 \cdot 9-93 \cdot 6)\end{array}$ & $\begin{array}{c}91 \cdot 7 \\
(89 \cdot 7-93 \cdot 8)\end{array}$ \\
\hline \multicolumn{11}{|l|}{ Oceania } \\
\hline \multicolumn{11}{|c|}{ Australia (eight registries)* } \\
\hline $2000-04$ & $\begin{array}{l}87.0 \\
(86.5-87.4)\end{array}$ & $\begin{array}{l}67 \cdot 9 \\
(66 \cdot 1-69 \cdot 6)\end{array}$ & $\begin{array}{l}37 \cdot 3 \\
(36 \cdot 1-38 \cdot 6)\end{array}$ & $\begin{array}{l}87 \cdot 8 \\
(87 \cdot 4-88 \cdot 2)\end{array}$ & $\begin{array}{l}24 \cdot 5 \\
(23 \cdot 5-25 \cdot 4)\end{array}$ & $\begin{array}{l}43 \cdot 3 \\
(42 \cdot 3-44 \cdot 3)\end{array}$ & $\begin{array}{l}61 \cdot 5 \\
(60 \cdot 9-62 \cdot 1)\end{array}$ & $\begin{array}{l}62 \cdot 0 \\
(57 \cdot 7-66 \cdot 3)\end{array}$ & $\begin{array}{l}86 \cdot 5 \\
(83 \cdot 8-89 \cdot 1)\end{array}$ & $\begin{array}{l}91 \cdot 4 \\
(88 \cdot 0-94 \cdot 8)\end{array}$ \\
\hline 2005-09 & $\begin{array}{l}88 \cdot 5 \\
(88 \cdot 1-89 \cdot 0)\end{array}$ & $\begin{array}{l}67 \cdot 5 \\
(65 \cdot 7-69 \cdot 3)\end{array}$ & $\begin{array}{l}41 \cdot 0 \\
(39 \cdot 8-42 \cdot 2)\end{array}$ & $\begin{array}{l}93 \cdot 2 \\
(92 \cdot 9-93 \cdot 5)\end{array}$ & $\begin{array}{l}28 \cdot 9 \\
(27 \cdot 9-29 \cdot 8)\end{array}$ & $\begin{array}{l}50 \cdot 3 \\
(49 \cdot 4-51 \cdot 1)\end{array}$ & $\begin{array}{l}68 \cdot 1 \\
(67 \cdot 6-68 \cdot 7)\end{array}$ & $\begin{array}{l}60 \cdot 6 \\
(56 \cdot 4-64 \cdot 9)\end{array}$ & $\begin{array}{l}90 \cdot 3 \\
(87 \cdot 9-92 \cdot 6)\end{array}$ & $\begin{array}{l}94 \cdot 2 \\
(91 \cdot 4-96 \cdot 9)\end{array}$ \\
\hline $2010-14$ & $\begin{array}{l}89.5 \\
(89.1-90.0)\end{array}$ & $\begin{array}{l}66 \cdot 4 \\
(64 \cdot 5-68 \cdot 2)\end{array}$ & $\begin{array}{l}42 \cdot 0 \\
(40 \cdot 8-43 \cdot 2)\end{array}$ & $\begin{array}{l}94 \cdot 5 \\
(94 \cdot 1-94 \cdot 8)\end{array}$ & $\begin{array}{l}30 \cdot 2 \\
(29 \cdot 2-31 \cdot 2)\end{array}$ & $\begin{array}{l}51 \cdot 8 \\
(50 \cdot 9-52 \cdot 7)\end{array}$ & $\begin{array}{l}71 \cdot 2 \\
(70 \cdot 6-71 \cdot 8)\end{array}$ & $\begin{array}{l}67 \cdot 1 \\
(62 \cdot 9-71 \cdot 4)\end{array}$ & $\begin{array}{l}90 \cdot 7 \\
(88 \cdot 3-93 \cdot 1)\end{array}$ & $\begin{array}{c}92 \cdot 3 \\
(89 \cdot 2-95 \cdot 4)\end{array}$ \\
\hline \multicolumn{11}{|c|}{ New Zealand* } \\
\hline $2000-04$ & $\begin{array}{l}82 \cdot 8 \\
(81 \cdot 6-84 \cdot 1)\end{array}$ & $\begin{array}{l}67 \cdot 4 \\
(63 \cdot 8-71 \cdot 1)\end{array}$ & $\begin{array}{l}38 \cdot 7 \\
(36 \cdot 0-41 \cdot 4)\end{array}$ & $\begin{array}{l}89 \cdot 1 \\
(88 \cdot 1-90 \cdot 0)\end{array}$ & $\begin{array}{l}19 \cdot 0 \\
(16 \cdot 8-21 \cdot 2)\end{array}$ & $\begin{array}{l}40 \cdot 3 \\
(37 \cdot 8-42 \cdot 9)\end{array}$ & $\begin{array}{l}61 \cdot 0 \\
(59 \cdot 6-62 \cdot 5)\end{array}$ & $\begin{array}{l}60 \cdot 2 \\
(50 \cdot 6-69 \cdot 8)\end{array}$ & $\begin{array}{l}85 \cdot 8 \\
(79 \cdot 9-91 \cdot 6)\end{array}$ & $\begin{array}{l}93.6 \\
(88 \cdot 7-98 \cdot 4)\end{array}$ \\
\hline 2005-09 & $\begin{array}{l}86 \cdot 1 \\
(84 \cdot 9-87 \cdot 3)\end{array}$ & $\begin{array}{l}64 \cdot 4 \\
(60 \cdot 4-68 \cdot 4)\end{array}$ & $\begin{array}{l}33 \cdot 4 \\
(31 \cdot 0-35 \cdot 9)\end{array}$ & $\begin{array}{l}89 \cdot 3 \\
(88 \cdot 4-90 \cdot 2)\end{array}$ & $\begin{array}{l}22 \cdot 7 \\
(20 \cdot 4-25 \cdot 0)\end{array}$ & $\begin{array}{l}49 \cdot 7 \\
(47 \cdot 7-51 \cdot 8)\end{array}$ & $\begin{array}{l}63 \cdot 5 \\
(62 \cdot 1-64 \cdot 9)\end{array}$ & $\begin{array}{l}54 \cdot 4 \\
(42 \cdot 9-66 \cdot 0)\end{array}$ & $\begin{array}{l}91 \cdot 2 \\
(86 \cdot 8-95 \cdot 6)\end{array}$ & $\begin{array}{l}93.5 \\
(88.0-99 \cdot 0)\end{array}$ \\
\hline 2010-14 & $\begin{array}{l}87 \cdot 6 \\
(86 \cdot 4-88 \cdot 7)\end{array}$ & $\begin{array}{l}67 \cdot 4 \\
(63 \cdot 4-71 \cdot 5)\end{array}$ & $\begin{array}{l}36 \cdot 7 \\
(34 \cdot 1-39 \cdot 3)\end{array}$ & $\begin{array}{l}90 \cdot 3 \\
(89 \cdot 4-91 \cdot 1)\end{array}$ & $\begin{array}{l}23 \cdot 3 \\
(21 \cdot 0-25 \cdot 7)\end{array}$ & $\begin{array}{l}44 \cdot 1 \\
(42 \cdot 1-46 \cdot 2)\end{array}$ & $\begin{array}{l}65 \cdot 6 \\
(64 \cdot 3-66 \cdot 9)\end{array}$ & $\begin{array}{l}58 \cdot 2 \\
(46 \cdot 1-70 \cdot 4)\end{array}$ & $\begin{array}{l}91 \cdot 4 \\
(86 \cdot 7-96 \cdot 1)\end{array}$ & $\begin{array}{l}96 \cdot 6 \\
(92 \cdot 7-100 \cdot 0)\end{array}$ \\
\hline \multicolumn{11}{|c|}{$\begin{array}{l}\text { Some registries contributed data for selected cancers or calendar periods. *Data with } 100 \% \text { coverage of the national population. †Survival estimate is not age-standardised. } \neq \text { Data with } 100 \% \text { coverage of th } \\
\text { national population for childhood malignancies only. SSurvival estimate considered less reliable because } 15 \% \text { or more of patients were (1) lost to follow-up or censored alive within } 5 \text { years of diagnosis or, if } \\
\text { diagnosed in } 2010 \text { or later, before Dec } 31,2014 \text {; or (2) registered only from a death certificate or at autopsy; or (3) patients with unknown vital status or registered with incomplete dates-ie, unknown year } \\
\text { of birth, unknown month or year of diagnosis, or unknown year of last known vital status. }\end{array}$} \\
\hline
\end{tabular}


Figure 4: Global range of age-standardised 5-year net survival estimates for colon cancer, breast cancer (women), and acute lymphoblastic leukaemia (children) among 296 cancer registry populations in 64 countries, by continent and calendar period of diagnosis

Each box plot shows the range of survival estimates among all cancer registries for which suitable estimates could be obtained for patients diagnosed in each calendar period, in each continent. Survival estimates considered less reliable are not included.

The vertical line inside each box represents the median survival estimate among all contributing registries (the central value in the range, or 50 th centile). The box covers

the IQR between the lower and upper quartiles (25th and 75th centiles). Where there are only a few widely scattered estimates, the median might be close to the lower or upper quartile. The extreme limits of the box plot are $1.5 \times I Q R$ below the lower quartile and $1.5 \times$ IQR above the upper quartile. Open circles indicate outlier values, outside this range. See appendix (pp 247-265) for other cancers.

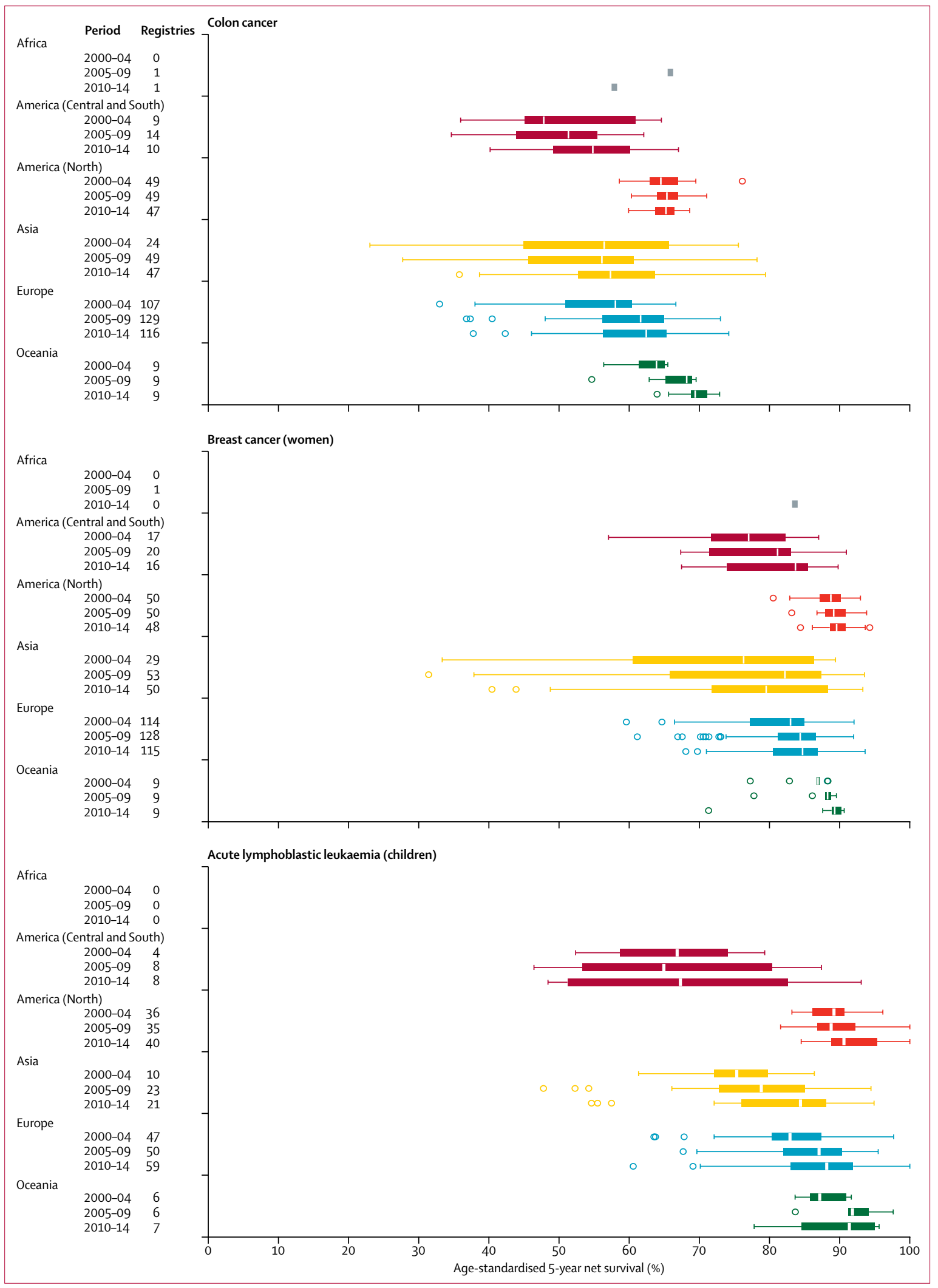


Europe]); and Australia and New Zealand (table 6; appendix p 217).

Survival was in the range $80-89 \%$ in 14 countries: Canada; Israel; and 12 European countries (Estonia, Finland, Iceland, Ireland, and Norway [northern Europe]; Italy, Malta, Portugal, Slovenia, and Spain [southern Europe]; the Czech Republic [eastern Europe]; and Austria [western Europe]).

Survival was in the range $70-79 \%$ in ten countries: four countries in Central and South America (Argentina, Brazil, Costa Rica, and Puerto Rico); and six European countries (Latvia and Lithuania [northern Europe]; Croatia [southern Europe]; and Poland, Romania [Cluj], and Slovakia [eastern Europe]).

Survival was in the range $60-69 \%$ in six countries: four Asian countries (Singapore [south Asia]; Korea and Japan [east Asia]; and Turkey [western Asia]); and Bulgaria and Russia. Survival was less than $60 \%$ in Ecuador, China, and Taiwan.

Trends between 2000-04 and 2010-14 were generally stable in North America, Oceania, and Japan, and in several European countries, where 5-year survival was already around 85-90\% among patients diagnosed during 2000-04. Survival increased by $5-10 \%$ in Korea and in 12 European countries (Denmark, Estonia, Latvia, Lithuania, and the UK [northern Europe]; Croatia, Portugal, and Slovenia [southern Europe]; Bulgaria, the Czech Republic, and Poland [eastern Europe]; and Belgium [western Europe]).

\section{Breast (women)}

Results are available for 6422553 women from 298 cancer registries in 66 countries (tables 2,5 ).

The range of survival estimates is still wide in each continent, apart from North America and Oceania (figure 4; appendix p 256). Most estimates were considered reliable (table 7; appendix pp 152-164).

For women diagnosed during 2010-14, age-standardised 5 -year net survival was $85 \%$ or higher in 25 countries: Costa Rica and Martinique; Canada and the USA; Israel, Japan, and Korea; 16 European countries (Denmark, Finland, Iceland, Norway, Sweden, and the UK [northern Europe]; Austria, Belgium, France, Germany, the Netherlands, and Switzerland [western Europe]; and Italy, Malta, Portugal, and Spain [southern Europe]); and Australia and New Zealand (table 7; figure 2; appendix p 218).

5-year survival was in the range $80-84 \%$ in 12 countries: three countries in Central and South America (Argentina, Peru [Lima], and Puerto Rico); five Asian countries (Singapore [south Asia]; China, Hong Kong, and Taiwan [east Asia]; and Turkey [west Asia]); and four European countries (Ireland [northern Europe]; the Czech Republic and Latvia [eastern Europe]; and Slovenia [southern Europe]). Survival was in the range 70-79\% in 12 countries: Cuba and Ecuador; Kuwait and Mongolia; and eight countries in Europe (Estonia and Lithuania [northern Europe]; Croatia [southern Europe]; and Bulgaria, Poland,
Romania [Clui], Russia, and Slovakia [eastern Europe]). Survival was still low in India (Karunagappally; table 7).

5-year net survival continued to increase up to 2010-14 in most countries in Central and South America, east and west Asia, and in all of Europe. Even so, survival remains lower in eastern Europe than in other parts of the continent. In North America and Oceania, 5-year net survival approached $90 \%$ (figure 3; appendix p 237).

\section{Cervix}

Results are available for 660744 women from 295 cancer registries in 64 countries (tables 2, 5). The global range in cervical cancer survival is still wide (50-70\%), especially in Central and South America, Asia, and Europe (table 7; appendix p 257). Most survival estimates are reliable (appendix pp 152-164).

For women diagnosed during 2010-14, agestandardised 5 -year net survival was $70 \%$ or higher in seven countries (Japan, Korea, and Taiwan; Denmark, Norway, and Switzerland; and Cuba), of which five had national coverage (table 7; appendix p 219).

Survival was in the range $60-69 \%$ in 29 countries: Canada and the USA; Brazil and Puerto Rico; five countries in Asia (China and Hong Kong [east Asia]; Singapore [south Asia]; and Israel and Turkey [west Asia]); 18 countries in Europe; and Australia and New Zealand.

Survival was in the range $50-59 \%$ in five countries in Central and South America (Argentina, Ecuador, Martinique, Peru [Lima], and Uruguay); India and Kuwait; and six European countries (Latvia and Lithuania [northern Europe]; Bulgaria, Poland, and Russia [eastern Europe]; and Malta [southern Europe]).

Over the 20 years from 1995 to 2014, 5-year survival has increased by 4-7\% in Cuba; Israel, Japan, and Korea; and six European countries (Denmark, Ireland, Lithuania, Norway, and the UK [northern Europe]; and Poland [eastern Europe]; appendix p 238). Survival increased by $8-10 \%$ in India; and in Bulgaria, Estonia, and Switzerland. In China, 5-year survival increased by $14.3 \%$ from 2000-04 to 2010-14 (table 7).

\section{Ovary}

Results are available for 865501 women from 289 registries in 61 countries (tables 2, 5).

Age-standardised 5-year net survival was mostly in the range $30-50 \%$, with even wider variation in Europe and Asia (appendix p 258). Most survival estimates were reliable (table 7; appendix pp 152-164).

For women diagnosed during 2010-14, 5-year survival was still less than $50 \%$ in most countries, except Costa Rica (table 7; appendix pp 220, 239, 258). Survival was in the range $40-49 \%$ in 24 countries: in Canada and the USA; seven countries in Asia (Singapore [south Asia]; China, Korea, Japan, and Taiwan [east Asia]; and Israel and Turkey [west Asia]); 14 European countries (Denmark, Estonia, Finland, Iceland, Latvia, Norway, and Sweden [northern Europe]; Portugal and Spain [southern 
Europe]; and Austria, Belgium, France, Germany, and Switzerland [western Europe]); and Australia.

Survival was in the range 30-39\% in 19 countries: four in Central and South America (Argentina, Brazil, Ecuador, and Puerto Rico); Kuwait and Thailand; 12 European countries (Ireland, Lithuania, and the UK [northern Europe]; Croatia, Italy, and Slovenia [southern Europe]; Bulgaria, the Czech Republic, Poland, Russia, and Slovakia [eastern Europe]; and the Netherlands [western Europe]); and New Zealand. Survival was less than $30 \%$ in Malta and less than $20 \%$ in India (Karunagappally; table 7).

Survival trends between 1995-99 and 2010-14 were fairly flat in most countries. However, 5-year survival rose by $5-10 \%$ in the USA; Israel, Korea, and Taiwan; 11 European countries (Denmark, Iceland, Ireland, Norway, and Sweden [northern Europe]; Portugal and Spain [southern Europe]; Bulgaria and Poland [eastern Europe]; and France and Switzerland [western Europe]); and Australia. Survival increased by more than $10 \%$ in Estonia and Latvia, and by $20 \%$ in Japan.

\section{Prostate}

Results are available for 5864878 men from 290 registries in 62 countries (tables 2, 5).

Age-standardised 5-year net survival was in the range $70-100 \%$ in most countries (appendix p 259). Most estimates were reliable (table 7; appendix pp 152-164).

For men diagnosed during 2010-14, 5-year survival was approaching $100 \%$ in Puerto Rico, Martinique, and the USA. Survival was at least $90 \%$ in a further 22 countries: Brazil and Costa Rica; Canada; Israel, Japan, and Korea; 14 European countries (Iceland, Ireland, Finland, Latvia, Lithuania, Norway, and Sweden [northern Europe]; Italy, Portugal, and Spain [southern Europe]; Austria, Belgium, France, and Germany [western Europe]); and Australia and New Zealand.

Survival was in the range $80-89 \%$ in 17 countries: Argentina, Ecuador, and Uruguay; five Asian countries (Malaysia [Penang] and Singapore [south Asia]; Taiwan [east Asia]; Kuwait and Turkey [west Asia]); and nine European countries (Denmark, Estonia, and the UK [northern Europe]; Croatia, Malta, and Slovenia [southern Europe]; the Czech Republic [eastern Europe]; and the Netherlands and Switzerland [western Europe]).

Survival approached $80 \%$ in Russia, Poland, and Romania (Cluj). It was less than $80 \%$ in Slovakia; less than $70 \%$ in China, Mauritius, Bulgaria, and Thailand; and less than $50 \%$ in India (Karunagappally; table 7).

Over the 20-year period between 1995-99 and 2010-14, age-standardised 5-year net survival rose in most countries. Survival increased by $5-10 \%$ in Brazil and Ecuador; Canada; China and Turkey; Austria and Portugal; and New Zealand. During the same period, 5-year survival rose by more than $10 \%$ in Israel, Taiwan, and Thailand, and in 12 European countries (Finland, Iceland, Norway, and Sweden [northern Europe]; Croatia, Italy, Malta, and Spain [southern Europe]; and France, Germany, the
Netherlands, and Switzerland [western Europe]); and Australia. Survival increased by more than $20 \%$ in 13 countries: Japan, Korea, and Malaysia (Penang); and ten European countries (Denmark, Estonia, Ireland, Latvia, Lithuania, and the UK [northern Europe]; Slovenia [southern Europe]; and Bulgaria, the Czech Republic, and Poland [eastern Europe]).

\section{Brain (adults)}

Results are available for 656659 adults from 286 registries in 59 countries (tables 2, 5). Age-standardised 5-year net survival was in the range $20-40 \%$ in most countries (appendix p 260). Most estimates were considered reliable (table 7; appendix pp 165-177).

For patients diagnosed during 2010-14, 5-year survival was higher than $40 \%$ only in Croatia (table 7). Survival was in the range $30-40 \%$ in 20 countries: Canada and the USA; Puerto Rico and Martinique; six Asian countries (Singapore [south Asia]; China and Korea [east Asia]; and Israel, Kuwait, and Turkey [west Asia]); nine European countries (Denmark, Estonia, Finland, Ireland, Norway, and Sweden [northern Europe]; and Belgium, Germany, and Switzerland [western Europe]); and Australia.

Survival was in the range $20-29 \%$ in 19 countries: Chile and Ecuador; Taiwan; 15 European countries (Iceland, Latvia, and the UK [northern Europe]; Italy, Malta, Portugal, Slovenia, and Spain [southern Europe]; the Czech Republic, Poland, Russia, and Slovakia [eastern Europe]; and Austria, France, and the Netherlands [western Europe]); and New Zealand (table 7). 5-year survival was $14 \cdot 7 \%$ in Thailand.

Trends in 5-year survival between 2000-04 and 2010-14 were generally rather flat, but survival increased by 3-5\% in 12 countries: Martinique; Canada; Israel; eight European countries (Iceland, Latvia, Norway, and Sweden [northern Europe]; Croatia and Italy [southern Europe]; and France and Switzerland [western Europe]); and New Zealand.

Survival increased by $6-10 \%$ in a further 12 countries: the USA; China, Korea, and Singapore; and seven European countries (Denmark, Estonia, Ireland, and the UK [northern Europe]; Malta and Spain [southern Europe]; and the Netherlands [western Europe]); and Australia.

\section{Myeloid malignancies}

Results are available for 1151226 adults from 286 registries in 61 countries (tables 2, 5).

Age-standardised 5-year net survival was in the range $30-50 \%$ in most countries, although lower in Asia (appendix p 261). Most estimates were considered reliable (table 7; appendix pp 165-177).

For patients diagnosed during 2010-14, survival was 55-60\% in Belgium, France, Germany, and Sweden; and in the range $50-54 \%$ in Canada; Turkey; eight European countries (Ireland, Lithuania, and Norway [northern Europe]; Portugal and Spain [southern Europe]; Romania [Cluj; eastern Europe]; and the Netherlands and Switzerland [western Europe]); and Australia. 
5-year survival was in the range 40-49\% in 11 countries: the USA; Martinique and Puerto Rico; Israel, Korea, and Singapore; four European countries (Denmark, Iceland, and the UK [northern Europe]; and Italy [southern Europe]); and New Zealand.

Survival was in the range $30-39 \%$ in 12 countries: Argentina and Brazil; Japan, Malaysia (Penang), and Taiwan; and seven European countries (Estonia [northern Europe]; Croatia and Slovenia [southern Europe]; the Czech Republic, Russia, and Slovakia [eastern Europe]; and Austria [western Europe]). Survival was less than $30 \%$ in Chile and Ecuador; China, Kuwait, and Thailand; and Latvia and Poland.

Over the 15 years between 2000-04 and 2010-14, agestandardised 5 -year net survival increased by $5-10 \%$ in 14 countries: the USA; China, Japan, Singapore, and Taiwan; eight European countries (Ireland and the UK [northern Europe]; Portugal and Spain [southern Europe]; Poland [eastern Europe]; and Austria, Belgium, and Germany [western Europe]); and Australia. Survival rose by more than $10 \%$ in Korea, Denmark, the Netherlands, and Norway.

Survival increased dramatically in Lithuania (27.4\%), Sweden (26.8\%), and the Czech Republic (16.9\%).

\section{Lymphoid malignancies}

Results are available for 3011054 adults from 289 registries in 62 countries (tables 2, 5).

5 -year age-standardised net survival was usually in the range $40-70 \%$ in most countries, but lower in Asia and in Central and South America (appendix p 262). Most estimates were considered reliable (table 7; appendix pp 165-177).

For patients diagnosed during 2010-14, 5-year survival was $70 \%$ or higher in six European countries (Denmark, Iceland, and Latvia [northern Europe]; and Belgium, France, and Switzerland [western Europe]); and Australia. Survival was in the range $60-69 \%$ in Mauritius; Puerto Rico; Canada and the USA; Israel and Kuwait; 12 European countries (Finland, Ireland, Norway, Sweden, and the UK [northern Europe]; Italy, Malta, Portugal, and Spain [southern Europe]; and Austria, Germany, and the Netherlands [western Europe]); and New Zealand.

Survival was $50-59 \%$ in 14 countries: Costa Rica; six Asian countries (Malaysia [Penang] and Singapore [south Asia]; Japan, Korea, and Taiwan [east Asia]; and Turkey [west Asia]); and seven European countries (Estonia and Lithuania [northern Europe]; Croatia and Slovenia [southern Europe]; and the Czech Republic, Poland, and Slovakia [eastern Europe]).

Survival was lower than $50 \%$ in five countries in Central and South America (Argentina, Brazil, Chile, Ecuador, and Martinique); China, India (Karunagappally), and Thailand; and Bulgaria, Romania (Cluj), and Russia.

5-year survival trends between 2000-04 and 2010-14 were increasing in most countries. Survival increased by
5-10\% in 23 countries: Ecuador; Canada and the USA; Japan and Taiwan; 15 European countries (Finland, Estonia, Iceland, and Sweden [northern Europe]; Croatia, Portugal, and Slovenia [southern Europe]; Bulgaria, the Czech Republic, Russia, and Slovakia [eastern Europe]; and Austria, Belgium, France, and Germany [western Europe]); and Australia and New Zealand.

Survival increased by more than $10 \%$ in 14 countries: Puerto Rico; Korea, Kuwait, and Singapore; and ten European countries (Denmark, Ireland, Latvia, Lithuania, Norway, and the UK [northern Europe]; Malta [southern Europe]; Poland [eastern Europe]; and the Netherlands and Switzerland [western Europe]).

\section{Brain (children)}

Results are available for 66814 children from 260 registries in 60 countries (tables 2, 5). Age-standardised 5-year net survival was in the range $40-80 \%$ in most countries (appendix p 263). Most estimates were reliable (table 7; appendix pp 165-177).

For children diagnosed during 2010-14, age-standardised 5-year net survival was close to $80 \%$ in Denmark, Slovakia, and Sweden. Survival was in the range $70-79 \%$ in 18 countries: Canada and the USA; Costa Rica and Puerto Rico; Israel and Japan; and 12 European countries (Finland, Ireland, Norway, and the UK [northern Europe]; Croatia, Italy, and Portugal [southern Europe]; the Czech Republic [eastern Europe]; and Belgium, France, Germany, and Switzerland [western Europe]).

Survival was in the range $60-69 \%$ in 14 countries: Korea, Singapore, and Turkey; ten European countries (Estonia, Latvia, and Lithuania [northern Europe]; Greece, Slovenia, and Spain [southern Europe]; Belarus, Poland, and Russia [eastern Europe]; and the Netherlands [western Europe]); and Australia. 5-year survival was less than $40 \%$ in Brazil and Mexico.

Survival trends between 2000-04 and 2010-14 were generally stable or increasing. 5-year age-standardised survival increased by $5-10 \%$ in the USA; China, Korea, and Turkey; six European countries (Ireland [northern Europe]; Croatia, Italy, and Portugal [southern Europe]; and Germany and the Netherlands [western Europe]); and Australia.

Survival increased by $10 \%$ or more in four European countries (Denmark and Lithuania [northern Europe]; and the Czech Republic and Slovakia [eastern Europe]).

\section{Acute lymphoblastic leukaemia (children)}

Results are available for 87351 children from 254 registries in 61 countries (tables 2, 5).

The global range in survival was very wide, from $50 \%$ to more than $90 \%$ (figure 2; appendix pp 226, 264). Most estimates were considered reliable. For children diagnosed during 2010-14, age-standardised 5-year net survival was $90 \%$ or more in Puerto Rico; Canada and the USA; eight European countries (Denmark, Finland, and the UK [northern Europe]; Portugal [southern Europe]; 
Belgium, Germany, the Netherlands, and Switzerland [western Europe]); and Australia and New Zealand.

Survival was in the range $80-89 \%$ in Costa Rica; six Asian countries (Singapore [south Asia]; Japan and Korea [east Asia]; and Israel, Kuwait, and Turkey [west Asia]); and 14 European countries (Estonia, Ireland, Latvia, Norway, and Sweden [northern Europe]; Croatia, Greece, Italy, and Spain [southern Europe]; Belarus, the Czech Republic, Poland, and Slovakia [eastern Europe]; and France [western Europe]; table 7; figure 2; appendix p 226).

5 -year net survival was still less than $70 \%$, even after adjustment for the very high background mortality in childhood, in Brazil, Chile, Colombia, and Peru (Lima). Survival was less than $60 \%$ in China, Ecuador, and Mexico.

In the 20-year period between 1995-99 and 2010-2014, 5 -year survival increased by $10 \%$ or more in 14 countries: Colombia; five Asian countries (China, Japan, Korea, and Taiwan [east Asia]; and Turkey [west Asia]); eight European countries (Finland, Lithuania, and the UK [northern Europe]; Portugal and Spain [southern Europe]; Belarus and Bulgaria [eastern Europe]; and Belgium [western Europe]; appendix p 245).

\section{Lymphoma (children)}

Results are available for 41196 children from 257 registries in 62 countries (tables 2, 5).

5-year age-standardised net survival was generally in the range $80-95 \%$ (table 7; appendix p 265). Most estimates were reliable. For children diagnosed during 2010-14, 5-year survival was at least $90 \%$ in 29 countries: Canada and the USA; Costa Rica and Puerto Rico; five Asian countries (Singapore [south Asia]; Japan and Korea [east Asia]; and Israel and Kuwait [west Asia]); 18 European countries (Denmark, Finland, Ireland, Lithuania, Norway, and the UK [northern Europe]; Croatia, Italy, Portugal, Slovenia, and Spain [southern Europe]; the Czech Republic, Poland, and Russia [eastern Europe]; and Belgium, France, Germany, and Switzerland [western Europe]); and Australia and New Zealand. 5-year survival was less than $70 \%$ only in Ecuador and China (table 7).

5 -year survival trends were generally rather flat over the 15 years between 2000-04 and 2010-14 (appendix p 246), but survival increased by $5-10 \%$ in the USA; Korea, Singapore, and Taiwan; and six European countries (the UK and Lithuania [northern Europe]; Portugal and Spain [southern Europe]; Slovakia [eastern Europe]; and Germany [western Europe]).

5 -year survival increased by more than $10 \%$ in Brazil, Bulgaria, Croatia, and Poland, and by at least $20 \%$ in Slovenia and Russia (table 7).

\section{Discussion}

CONCORD-3 updates the worldwide surveillance of cancer survival to 2014. It is the largest and most up-todate study of international cancer survival trends. It includes individual data for more than 37.5 million patients diagnosed with cancer during the 15 -year period
2000-14. Data were provided by more than 320 populationbased cancer registries in 71 countries and territories, in 47 of which the data covered $100 \%$ of the population. The participating countries were home to $67 \%$ of the world's population in 2014 ( 7.3 billion people).$^{28}$ The registries record all cancers diagnosed in a combined population of almost 1 billion people, or $14 \%$ of the world population. Internationally comparable survival trends are now available for 18 cancers that collectively represent $75 \%$ of all cancers diagnosed worldwide every year.

We used a similar design and statistical approach to those used in CONCORD- $2^{6}$ to enable evaluation of survival trends for ten cancers over the 20-year period 1995-2014. Worldwide survival trends are also available for the first time for melanoma of the skin and cancers of the oesophagus and pancreas in adults, and for brain tumours and lymphomas in both adults and children.

5 -year survival has been recognised by clinicians as an index of the effectiveness of the treatment of cancer for more than 60 years. When applied to hospital case series, it has often been labelled as the 5-year cure rate, because "with so mortal a disease as cancer, those who survive for this length of time can be considered cured". ${ }^{44} 5$-year survival has increased for many cancers since the 1950s, but it remains a widely used benchmark, even though it cannot be directly interpreted as the proportion of patients who are cured. ${ }^{45}$

Population-based cancer survival is increasingly recognised as a key indicator of the overall effectiveness of health systems in managing care and treatment for all patients with cancer. ${ }^{46,47}$ Other outcome measures with applications in cancer control include the number of avoidable premature deaths, ${ }^{48-53}$ person-years of life lost, ${ }^{54,55}$ disability-adjusted life-years $\operatorname{lost}^{56}$ and estimates of the proportion of patients with cancer who can be considered to have been cured. ${ }^{57-61}$ Cancer survival has applications to cancer control and health policy at the state, national, and global levels, in both high-income and low-income countries. ${ }^{62}$

In some countries, population-based cancer survival estimates might be considered as too high, potentially discouraging ministerial action to improve survival. Estimates showing ethnic or regional variation in cancer survival can be politically sensitive. Survival estimates can also be considered too low if they are seen as a reflection of clinical competence. ${ }^{63}$ Low levels of survival in a country or region should not be interpreted as an indicator of the competence of the health professionals who work there. Population-based survival reflects the overall effectiveness of the health service, which depends on much wider issues than the competence of any individual doctor or team..$^{64-66}$

The Organisation for Economic Co-operation and Development (OECD) recently concluded from the wide international variation in cancer survival that many countries could do better in cancer control. ${ }^{64}$ It recommended a national cancer plan, adequate funding, 
and initiatives for early detection and rapid access to high-quality treatment. ${ }^{64}$ The OECD also recommended improving the quality of cancer data to support monitoring improvements in survival.

From 2017, the OECD has included age-standardised 5 -year net survival estimates from the CONCORD programme for colorectal and breast cancers in adults and acute lymphoblastic leukaemia in children among the indicators of health-care quality in its biennial and online publications Health at a Glance. ${ }^{67}$ This is formal recognition of the global coverage, methodological rigour, and international comparability of the CONCORD survival estimates, which will now contribute to the comparative evaluation of health systems performance in 48 countries, including all OECD member countries. The findings will also help to monitor progress toward the overarching goal of the 2013 World Cancer Declaration: to achieve major improvements in cancer survival by $2020 .^{68}$

We carried out extensive checks on data quality and liaised with the cancer registries to resolve problems. Many registries told us that the CONCORD data quality reports helped them to improve their data. Some centres in Nigeria and India have modified hospital admission forms or pathology request forms to capture telephone numbers of patients and their next of kin, to facilitate follow-up of their patients. We extended our programs and reports on data quality control. Rectifying errors or inconsistencies in the data often led to extensive discussion with registry staff and resubmission of data with higher quality.

The quality and completeness of cancer registration data and follow-up vary between countries, and this can affect the comparability of survival estimates. We have provided extensive documentation of data quality with standard indicators ${ }^{69}$ for each cancer and each cancer registry (appendix pp 6-101). Survival figures and trends should be interpreted alongside those indicators. The overall proportion of tumour records excluded because of incomplete dates $(0.5 \%)$ or for other reasons such as missing vital status $(1 \cdot 2 \%)$ has remained very low. The overall proportion of cancers registered solely from a DCO or detected at autopsy dropped to $2.9 \%$, but remains high in some countries where cancer registration processes are slow, especially for the more rapidly lethal cancers of the oesophagus, pancreas, and liver. These are well known issues in population-based cancer registration. DCOs can be included in cancer incidence statistics under certain assumptions, but they reflect some underestimation of incidence. ${ }^{69}$ By contrast, DCO cases must be excluded from survival analyses, because the patient's survival time is unknown: this tends to inflate survival estimates. ${ }^{70}$

In some countries, survival estimates have fluctuated or declined in successive calendar periods; this is likely to reflect improvements in the completeness of cancer registration data and in the completeness of follow-up for vital status. In Jordan, for example, linkage with the national death index has been insufficient because only about $70 \%$ of deaths are certified. Survival estimates were very high for stomach and colorectal cancers and are flagged as less reliable. A recent hospital-based survival study $^{71}$ from Jordan suggests that colon cancer survival in Jordan is much lower than the estimates we have obtained. Other countries with incomplete death registration, such as India, follow up their patients actively to determine their vital status.

Despite these problems, we believe our findings represent the best that can be achieved with the available coverage and quality of cancer registration systems and vital statistics systems worldwide. The quality of diagnostic evidence is generally high. Data quality has improved in many countries, with increasing proportions of cases for which the diagnosis was confirmed by pathology, imaging, or biomarkers, and a reduction in the proportion of patients lost to follow-up. Pathological confirmation of a primary, invasive malignancy was available for more than $94 \%$ of all patients. The proportion varies widely between countries and for different cancers, but the evidence supporting a cancer diagnosis in routine cancer registry data is far more precise and definitive than for the cause or causes of death recorded on death certificates, from which mortality statistics are derived. ${ }^{72-75}$

In some cases, especially in South America, the improvement in data quality is reflected in survival estimates that are actually lower than those previously published, ${ }^{6}$ with fewer DCO registrations and more complete follow-up. In Brazil, for example, improvement in the quality and completeness of the national death registry $^{76}$ during 2000-15 has enabled more complete linkage of death records with the cancer registries. In several countries, survival estimates that were flagged as less reliable in CONCORD-2 are now more reliable, even if the survival estimates are lower.

To estimate the global burden of cancer incidence, assumptions are required where no cancer registries exist, usually by modelling incidence and mortality data from other countries in the same world region., 2,7 By contrast, we have made no attempt to model cancer survival in countries or regions where population-based cancer registration data were not available. Cancer survival cannot be estimated or modelled by assuming that the health system is as effective as in some other country for which population-based survival estimates are available. On the contrary, cancer survival estimates are required to assess the overall effectiveness of a country's health system in the first place. For that, cancer registries are essential.

The survival estimates reported here are derived directly from the records of individual patients diagnosed with cancer, and from long-term follow-up to ascertain their vital status, followed by standardised quality control and central analysis. This is not a compilation of published reports or a meta-analysis. 
Survival for most cancers remains among the highest in the world in the USA, Canada, Australia, and New Zealand, and in Finland, Iceland, Norway, and Sweden. Publications that showed surprisingly low survival in Denmark $^{78,79}$ prompted national cancer plans in 2000 , 2005, and 2011 that were all focused on early diagnosis and treatment to improve survival. ${ }^{80}$ From 2007, cancer was regarded as an acute life-threatening disease, leading to accelerated cancer-specific pathways for diagnosis, with public monitoring of hospitals' compliance with waiting times. For most cancers, survival has increased more rapidly in Denmark, nearly catching up with the other Nordic countries..$^{81,82}$ Norway and Sweden have now established similar pathways for cancer patients.

Cancer survival trends are generally increasing, even for some of the more lethal cancers. In some countries, survival increased by up to $5 \%$ for cancers of the liver, pancreas, or lung. For example, survival trends for liver cancer were generally stable during 1995-2014, but survival increased by more than $10 \%$ in Korea, Singapore, and Norway.

However, for cancers for which 5-year survival remains extremely low in all countries (eg, pancreatic cancer, in the range 5-15\%), international efforts will be required to understand risk factors, improve prevention, and promote earlier diagnosis and better treatment to improve outcomes. International comparisons of survival for pancreatic cancer include both ductal adenocarcinomas and the less common neuroendocrine tumours, for which survival is generally higher. The effect of variation in these proportions will require detailed analysis.

Age-standardised 5-year net survival for stomach cancer was less than $30 \%$ in most countries, but high in Korea $(68 \cdot 9 \%)$ and Japan $(60 \cdot 3 \%)$, where it increased by $10 \%$ or more between 2000-04 and 2010-14. This pattern is likely to be associated with long-standing population-based endoscopic screening programmes for early detection of gastric and oesophageal cancers, which are very common. Population awareness is high. Gastric cancer screening in Korea started in 1999 as part of the National Cancer Screening Programme, with biennial contrast radiology or endoscopy for adults aged 40 years or older ${ }^{83}$ This doubles the chances of early diagnosis compared with unscreened patients.$^{84}$ Endoscopic resection with clear margins can be curative in stage I oesophageal and gastric cancers (up to $2 \mathrm{~cm}$ diameter) if invasion is limited to the superficial submucosa and there is no lymphovascular invasion. ${ }^{85}$ By contrast, in countries where gastric cancer is a less serious public health issue, and in the absence of screening, this cancer is often diagnosed at an advanced stage. In Russia in 2015, for example, stomach cancer was most often diagnosed in stage IV (40\%), and open laparotomy, chemotherapy, and radiation were required for $22 \%$ and $25 \%$ of cases in stages II and III, respectively. Screening for oesophageal and gastric cancers should be considered as part of national cancer control plans in countries where these tumours are common, or in high-risk populations. ${ }^{86}$
Survival trends for colorectal cancer were generally flat, or increasing, over the 20 years 1995-2014. Survival for rectal cancer was very similar among the Nordic countries (64-69\%) and among most southern European countries (61\%).

Survival from melanoma of the skin is generally lower in Asian populations than in the rest of the world. One explanation might be lower public awareness because melanoma is less common in Asian populations, but it could also be that Asian patients typically present with more advanced disease and with acral lentiginous melanoma. This is one of the more lethal subtypes, and it is more common in Asian populations than in western populations. ${ }^{87}$ In the CONCORD data (not shown), acral lentiginous melanoma represents $1 \cdot 2 \%$ of all skin melanomas, and 1\% in Europe and North America, but $6 \%$ in Asia.

The increasing trend in 5-year net survival from breast cancer during the 15 years $1995-2009^{6}$ has continued in most countries up to 2014, but remains lower in India, Thailand, and several of the eastern European countries.

5 -year survival from cervical cancer has increased in several European and Asian countries. However, survival can even decline following an increase in diagnostic activity. In North America and Oceania, survival is lower than in other countries due to more intensive screening programmes that detect precancerous cells and in-situ tumours. These can be cured with a range of simple techniques, reducing the incidence of invasive malignancy by removal of the more indolent pre-invasive lesions, whereas the more aggressive tumours are less likely to be detected by screening.

Some of the global range in survival might be attributable to differences in the intensity of diagnostic activity, and to overdiagnosis from the detection of very small or less aggressive tumours that would not have been expected to lead to symptomatic diagnosis or death in the patient's expected lifetime. ${ }^{88,89}$ As in CONCORD- $2,{ }^{6}$ we were unable to use the proportion of in-situ cancers to compare the intensity of diagnostic activity for solid tumours. Some registries still do not record in-situ tumours and other registries did not submit data for in-situ tumours.

Because screening programmes are only available in wealthier countries, mainly in selected age ranges for cancers of the breast, cervix, and colon, the extent of overdiagnosis seems unlikely to have a large effect on the global range of cancer survival. Measures of overdiagnosis are only available at the population level, so their application in the interpretation of cancer survival patterns would be limited to ecological comparisons, as for gross domestic product (GDP) or total national expenditure on health. By contrast, data on stage at diagnosis are available for individuals. Analyses of the distribution of stage at diagnosis and stage-specific survival will be expected to provide further insight into international variation in cancer survival..$^{90,91}$ 
Survival from the adult leukaemias up to 2009 in Asian populations was much lower than in Europe, North America, and Oceania. ${ }^{6}$ One possible explanation was the relative rarity in Asian populations of chronic lymphocytic leukaemia, which has a relatively good prognosis in western populations. However, survival from chronic lymphocytic leukaemia is also much lower in Taiwan than in the USA, ${ }^{92}$ and the findings reported here also show that survival in adults is generally lower in southeast Asia than in other countries, for both myeloid and lymphoid malignancies. The difference between the median of the survival estimates for Asian populations and for other populations narrowed between 2000-04 and 2010-14, for both myeloid and lymphoid malignancies. In most southeast Asian countries, survival for myeloid malignancies has risen by $5-14 \%$, and by $10 \%$ or more for lymphoid malignancies.

Survival from brain tumours in children is generally higher than for adults, but the global range is much more pronounced. Some of the international variation in survival from brain tumours might be due to variation in the proportion that are benign. Where benign tumours are registered, the proportion typically ranges up to $10-15 \%$ in both adults and children (data not shown). However, some registries do not record benign brain tumours, and this varies both between and within countries. For example, in Australia, benign brain tumours are not registrable in New South Wales or Western Australia (45\% of the national population), whereas they comprise up to $5 \%$ of brain tumours in Queensland and Victoria, with a similar combined population. The impact of morphology, behaviour, and grade on international patterns of brain tumour survival needs further research.

International variation in survival for childhood lymphoma was less marked than for childhood acute lymphoblastic leukaemia. The marked increase in 5-year survival among children diagnosed with lymphoma in Brazil (from $69 \cdot 2 \%$ in $2000-04$ to $88 \cdot 2 \%$ in $2010-14$ ) is likely to reflect a real improvement in diagnosis and treatment.

Cancer kills more than 100000 children every year, mainly in low-income and middle-income countries, ${ }^{93}$ where access to health services is often poor and abandonment of treatment is a major problem. ${ }^{94,95}$ Reliable data on the cost and the effectiveness of health services in managing childhood cancer are scarce, yet such data would offer important evidence for countries to compare the impact of their strategies for managing children with cancer. ${ }^{96}$ Survival estimates published here for children diagnosed with a brain tumour, lymphoma, or leukaemia will be used in a Lancet Oncology Commission on childhood cancer, designed to establish the evidence for investing in effective interventions to reduce the burden of childhood cancer.

Survival trends could not be systematically assessed in Africa. In some registries, the proportion of records with incomplete dates reached up to $40 \%$. Survival estimates for acute lymphoblastic leukaemia in Algeria were considered less reliable because follow-up was less than 5 years for more than $50 \%$ of children. For Nigeria (Ibadan) and South Africa (Eastern Cape), data were only available for 12 and seven children, respectively, and survival was not estimated. Where survival could be estimated with some confidence, it was often very low, although survival in Mauritius was generally higher. In Nigeria, for example, there are no trained medical oncologists. Some haematologists and paediatric oncologists administer chemotherapy, but the availability of chemotherapy is limited in both the public and private sectors. Most patients pay out of their own pocket, and the cost is prohibitive. These factors frequently disrupt treatment and are likely to lead to poor outcomes.

To control for background mortality by age and sex, we updated the library of life tables for 1995-2010 by country, registry, race (selected countries), and calendar year to 2014, with a statistical summary for each set of life tables. The updated library is available from the Cancer Survival Group website. In some countries, it has become more difficult to obtain the death and population counts required to construct life tables.

Survival estimates from CONCORD-2 for cancers of the breast and cervix were used in a recent Lancet Series on women's cancers, ${ }^{97,98}$ to help to describe trends in the global burden of these cancers. Survival for the 2 million women diagnosed with one of these cancers every year remains highly dependent on the country in which they live. The Series highlighted the urgent need for more cost-effective cancer control strategies in low-income and middle-income countries.

The global economic cost of treating the 12.9 million new patients diagnosed with cancer worldwide in 2009 was estimated at US $\$ 285.8$ billion..$^{99}$ The costs of cancer treatment and care in the USA alone were projected to rise by $23 \%$ between 2010 and 2020, from $\$ 124.6$ to $\$ 157.7$ billion, solely on the basis of demographic change, and with fixed incidence rates, survival probabilities, and treatment costs. ${ }^{100}$ If treatment costs rise by $2 \%$ a year in the first and last phases of treatment, the overall cost of treatment and care in the USA could reach $\$ 172.8$ billion, a $39 \%$ increase. If incidence rates continue to rise, the prevalence of cancer survivors will increase further, triggering still further increase in the costs of care.

On the basis of these figures, it seems plausible that the global cost of cancer treatment and care in 2017 must already be substantially higher than $\$ 300$ billion a year. Spiralling costs ${ }^{101}$ threaten the viability of health systems and national economies. Where universal health coverage has not been achieved, the out-of-pocket costs of cancer treatment can lead to financial catastrophe for individuals and families. ${ }^{3}$

The indirect economic costs associated with premature death and lost productivity from the growing cancer
For the life tables from the CONCORD programme see http://csg.lshtm.ac.uk/toolsanalysis\#tools 
burden have been estimated at $\$ 1.16$ trillion a year, ${ }^{3}$ or approaching $2 \%$ of global GDP. ${ }^{102}$ The increasing cost and complexity of cancer treatment might require a radical shift in cancer policy, in which inequitable access to affordable cancer treatment ceases to be politically acceptable. ${ }^{103}$ Population-based data on cancer survival trends that are comparable within and between countries are part of the evidence base needed to drive such a policy shift.

With this background, cancer registries can be seen as efficient public health instruments, producing a continuous stream of valuable information for cancer control at low cost. ${ }^{104}$ In Europe in 2013, the average cost per patient registered, including the registry's costs for personnel, information technology, and infrastructure, was $€ 51$ (range €6-213; equivalent to \$59 [7-252]). This is less than the typical cost of a chest $\mathrm{x}$-ray. For the population as a whole, the cost was less than $€ 1(\$ 1 \cdot 18)$ per person per year.

In 2015, the UN introduced 17 Sustainable Development Goals (SDGs), aiming to end poverty, protect the planet, and ensure prosperity for all. ${ }^{105}$ Goal 3 is "to ensure healthy lives and promote well-being for all at all ages". For this goal, target 3.4 is to reduce "premature" mortality (among people aged 30-70 years) from non-communicable diseases, including cancer, by a third by 2030 , through prevention and treatment. ${ }^{106}$ The challenge will be to secure overall improvements in health outcomes that do not lead to wider inequalities. ${ }^{107}$

Achieving the SDG target of a one-third reduction in premature mortality by 2030 clearly requires more effective prevention to reduce cancer incidence. However, the 15-year timeframe is short, and achieving the target will also require investment in more effective health systems, to improve survival. ${ }^{46}$

WHO recently called for the development of populationbased cancer registries, so that effective policies for cancer control can be founded on accurate data. ${ }^{3}$ It also called for stronger civil registration and vital statistics systems. These systems support the basic functions of government and enable measurement of progress towards development goals, ${ }^{108}$ yet population coverage is poor in low-income and middle-income countries, and closely related to gross national income. ${ }^{109}$

Most cancer registries establish the vital status of all patients registered with cancer by linkage with vital statistics data (regional or national death indexes). This is known as passive follow-up, although many registries also contact patients' doctors or families directly (active follow-up). For passive follow-up to work, efficient civil registration systems that capture information on all deaths are required. This process underpins the estimation of population-based cancer survival, even though active follow-up can be effective in some populations. Yet cancer registries report increasing difficulty in linking their databases to regional or national death indexes. Legal and administrative obstacles and technical difficulties have all been reported. Some national authorities holding death indexes clearly give very low priority to such linkages.

These problems undermine the public health purpose of cancer registration. Of the 400 operational registries we contacted, more than 20 were unable to follow up all registered patients to ascertain their vital status. This problem arose in 16 countries, including some highincome countries. Some registries were unable to provide survival data at all. In Canada, for example, national coverage of cancer survival statistics was achieved for the first time in CONCORD-2, ${ }^{6}$ with data from all 13 provinces and territories for 1995-2009, but several jurisdictions were unable to participate in CONCORD-3 because of legal or administrative difficulties in linking their cancer registry with death records. For eight countries that expressed interest or even submitted data, these difficulties meant that no survival estimates could be produced at all: Benin, Bosnia and Herzegovina, Indonesia, Panama, the Philippines, Saudi Arabia, Serbia, and Tunisia.

It is crucial for national and regional governments to recognise that population-based cancer registries are key policy tools, both to monitor the impact of cancer prevention strategies and to evaluate the effectiveness of the health system for all patients diagnosed with cancer. All registries, especially those in low-income and middleincome countries, need to be given adequate resources to register all patients with cancer in a timely fashion, the right to access up-to-date national and regional death records to establish their vital status, and the legislative stability to operate efficiently over the long term. ${ }^{10}$

\section{Contributors}

$\mathrm{CA}, \mathrm{AB}, \mathrm{CAS}$, and MPC drafted the protocol. CA and MPC obtained statutory and ethical approvals. GAeS, W-QC, GE, SE, CJJ, GHL, AM, TM, OJO, MV, and HKW contributed to data acquisition. CA, VDC, and MPC prepared the life tables. CA, VDC, RH, MM, MN, AB, and MPC had access to all the raw data. CA, VDC, RH, MM, MN, AB, CJJ, and MPC did the data preparation, quality control, and analyses, and CA VDC, RH, MM, MN, AB, JA, GE, JE, SE, OV, RW, and MPC checked the results. CA and MPC drafted the report. All authors contributed to writing the final report and approved the version to be published. All members of the CONCORD Working Group had access to the results of all steps of data preparation, quality control, and analyses, and contributed to interpretation of the findings.

\section{CONCORD Working Group}

Africa: Algeria: S Bouzbid (Registre du Cancer d'Annaba);

M Hamdi-Chérif*, Z Zaidi (Registre du Cancer de Sétif); K Meguenni, D Regagba (Registre du Cancer Tlemcen); Mali: S Bayo, T Cheick Bougadari (Kankou Moussa University); Mauritius: S S Manraj (Mauritius National Cancer Registry); Morocco: K Bendahhou (Registre du Cancer du Grand Casablanca); Nigeria: A Fabowale, O J Ogunbiyi* (Ibadan Cancer Registry); South Africa: D Bradshaw, N I M Somdyala (Eastern Cape Province Cancer Registry). America (Central and South): Argentina: I Kumcher, F Moreno (National Childhood Cancer Registry); G H Calabrano, S B Espinola (Chubut Cancer Registry); B Carballo Quintero, R Fita (Registro Provincial de Tumores de Córdoba); M C Diumenjo, W D Laspada (Registro Provincial de Tumores de Mendoza); S G Ibañez (Population Registry of Cancer of the Province Tierra del Fuego); Brazil: C A Lima (Registro de Câncer de Base Populacional de Aracaju); P C F De Souza (Registro de Câncer de Base Populacional de Cuiabá); K Del Pino, C Laporte (Registro de Curitiba); M P Curado, J C de Oliveira (Registro de Goiânia); C L A Veneziano, 
D B Veneziano (Registro de Câncer de Base Populacional de Jaú); M R D O Latorre, L F Tanaka (Registro de Câncer de São Paulo); M S Rebelo, M O Santos (Instituto Nacional de Câncer, Rio de Janeiro); G Azevedo e Silva* (University of Rio de Janeiro); Chile: J C Galaz (Registro Poblacional de Cáncer Region de Antofagasta); M Aparicio Aravena, J Sanhueza Monsalve (Registro Poblacional de Cáncer de la Provincia de Biobio; Registro Poblacional de Cáncer Provincia de Concepción); D A Herrmann, S Vargas (Registro Poblacional Region de Los Rios); Colombia: V M Herrera, C J Uribe (Registro Poblacional de Cáncer Area Metropolitana de Bucaramanga); L E Bravo, L S Garcia (Cali Cancer Registry); N E Arias-Ortiz, D Morantes (Registro Poblacional de Cáncer de Manizales); D M Jurado, M C Yépez Chamorro (Registro Poblacional de Cáncer del Municipio de Pasto); Costa Rica: S Delgado, M Ramirez (Registro Nacional de Tumores de Costa Rica); Cuba: Y H Galán Alvarez, P Torres (Registro Nacional de Cáncer de Cuba); Ecuador: F Martínez-Reyes (Cuenca Tumor Registry); L Jaramillo, R Quinto (Guayaquil Cancer Registry); J Castillo (Loja Cancer Registry); M Mendoza (Manabí Cancer Registry); P Cueva, J G Yépez (Quito Cancer Registry); France: B Bhakkan, J Deloumeaux (Registre des cancers de la Guadeloupe); C Joachim, J Macni (General Cancer Registry of Martinique); Mexico: R Carrillo, J Shalkow Klincovstein (Centro Nacional para la Salud de la Infancia y la Adolescencia); R Rivera Gomez (Registro Poblacional de Cancer Region Fronteriza Norte de Mexico Zona Tijuana); Peru: E Poquioma (Lima Metropolitan Cancer Registry); Puerto Rico: G Tortolero-Luna, D Zavala (Puerto Rico Central Cancer Registry); Uruguay: R Alonso, E Barrios (Registro Nacional de Cáncer). America (North): Canada: A Eckstrand, C Nikiforuk (Alberta Cancer Registry); R R Woods (British Columbia Cancer Registry); G Noonan, D Turner* (Manitoba Cancer Registry); E Kumar, B Zhang (New Brunswick Provincial Cancer Registry); F R McCrate, S Ryan (Newfoundland \& Labrador Cancer Registry); M MacIntyre, N Saint-Jacques (Nova Scotia Cancer Registry); D E Nishri* (Ontario Cancer Registry); C A McClure, K A Vriends (Prince Edward Island Cancer Registry); S Kozie, H Stuart-Panko (Saskatchewan Cancer Agency); USA: T Freeman, J T George (Alabama Statewide Cancer Registry); J T Brockhouse, D K O’Brien (Alaska Cancer Registry); A Holt (Arkansas Central Cancer Registry); L Almon (Metropolitan Atlanta Registry); S Kwong, C Morris (California State Cancer Registry); R Rycroft (Colorado Central Cancer Registry); L Mueller, C E Phillips (Connecticut Tumor Registry); H Brown, B Cromartie (Delaware Cancer Registry); A G Schwartz, F Vigneau (Metropolitan Detroit Cancer Surveillance System); G M Levin, B Wohler (Florida Cancer Data System); R Bayakly (Georgia Cancer Registry); K C Ward (Georgia Cancer Registry; Metropolitan Atlanta Registry); S L Gomez, M McKinley (Greater Bay Area Cancer Registry); R Cress (Cancer Registry of Greater California); M D Green, K Miyagi (Hawaii Tumor Registry); C J Johnson (Cancer Data Registry of Idaho); L P Ruppert (Indiana State Cancer Registry); C F Lynch (State Health Registry of Iowa); B Huang, T C Tucker* (Kentucky Cancer Registry); D Deapen, L Liu (Los Angeles Cancer Surveillance Program); M C Hsieh, X C Wu (Louisiana Tumor Registry); M Schwenn (Maine Cancer Registry); S T Gershman, R C Knowlton (Massachusetts Cancer Registry); G Alverson, G E Copeland (Michigan State Cancer Surveillance Program); S Bushhouse (Minnesota Cancer Surveillance System); D B Rogers (Mississippi Cancer Registry); J Jackson-Thompson (Missouri Cancer Registry and Research Center); D Lemons, H J Zimmerman (Montana Central Tumor Registry); M Hood, J Roberts-Johnson (Nebraska Cancer Registry); J R Rees, B Riddle (New Hampshire State Cancer Registry); K S Pawlish, A Stroup (New Jersey State Cancer Registry); C Key, C Wiggins (New Mexico Tumor Registry); A R Kahn, M J Schymura (New York State Cancer Registry); S Radhakrishnan, C Rao (North Carolina Central Cancer Registry); L K Giljahn, R M Slocumb (Ohio Cancer Incidence Surveillance System); R E Espinoza, F Khan (Oklahoma Central Cancer Registry); K G Aird, T Beran (Oregon State Cancer Registry); J J Rubertone, S J Slack (Pennsylvania Cancer Registry); L Garcia, D L Rousseau (Rhode Island Cancer Registry); T A Janes, S M Schwartz (Seattle Cancer Surveillance System); S W Bolick, D M Hurley (South Carolina Central Cancer Registry); M A Whiteside (Tennessee Cancer Registry); P Miller-Gianturco, M A Williams (Texas Cancer Registry); K Herget, C Sweeney (Utah Cancer Registry); A T Johnson (Vermont Cancer Registry); M B Keitheri
Cheteri, P Migliore Santiago (Washington State Cancer Registry); S E Blankenship, S Farley (West Virginia Cancer Registry); R Borchers, R Malicki (Wisconsin Department of Health Services); J R Espinoza, J Grandpre (Wyoming Cancer Surveillance Program); H K Weir*, $\mathrm{R}$ Wilson (Centers for Disease Control and Prevention); B K Edwards*, A Mariotto (National Cancer Institute). Asia: China: Y Lei, N Wang (Beijing Cancer Registry); J S Chen, Y Zhou (Changle City Cancer Registry); Y T He, G H Song (Cixian Cancer Registry); X P Gu (Dafeng County Center for Disease Control and Prevention); D Mei, H J Mu (Dalian Centers for Disease Prevention and Control); H M Ge, T H Wu (Donghai County Center for Disease Prevention and Control); Y Y Li, D L Zhao (Feicheng County Cancer Registry); F Jin, J H Zhang (Ganyu Center for Disease Prevention and Control); F D Zhu (Guanyun Cancer Registry); Q Junhua, Y L Yang (Haimen Cancer Registry); C X Jiang (Haining City Cancer Registry); W Biao, J Wang (Jianhu Cancer Registry); Q L Li (Jiashan County Cancer Registry); H Yi, X Zhou (Jintan Cancer Registry); J Dong, W Li (Lianyungang Center for Disease Prevention and Control); F X Fu, S Z Liu (Linzhou Cancer Registry); J G Chen, J Zhu (Qidong County Cancer Registry); Y H Li, Y Q Lu (Sihui Cancer Registry); M Fan, S Q Huang (Taixing Cancer Registry); G P Guo, H Zhaolai (Cancer Institute of Yangzhong City); K Wei (Zhongshan City Cancer Registry); W Q Chen*, H Zeng (The National Cancer Center); Cyprus: A V Demetriou (Cyprus Cancer Registry); Hong Kong: W K Mang, K C Ngan (Hong Kong Cancer Registry); India: A C Kataki, M Krishnatreya (Guwahati Cancer Registry); P A Jayalekshmi, P Sebastian (Karunagappally Cancer Registry); A Nandakumar* (National Centre for Disease Informatics and Research); Iran: R Malekzadeh, G Roshandel (Golestan Population-based Cancer Registry); Israel: L Keinan-Boker, B G Silverman (Israel National Cancer Registry); Japan: H Ito, H Nakagawa (Aichi Cancer Registry); M Sato, F Tobori (Akita Prefectural Cancer Registry); I Nakata, N Teramoto (Ehime Prefectural Cancer Registry); M Hattori, Y Kaizaki (Fukui Cancer Registry); F Moki (Gunma Prefectural Cancer Registry); H Sugiyama, M Utada (Hiroshima Prefecture Cancer Registry); M Nishimura, K Yoshida (Hyogo Prefectural Cancer Registry); K Kurosawa, Y Nemoto (Ibaraki Prefectural Cancer Registry); H Narimatsu, M Sakaguchi (Kanagawa Cancer Registry); S Kanemura (Miyagi Prefectural Cancer Registry); M Naito, R Narisawa (Niigata Prefecture Cancer Registry); I Miyashiro, K Nakata (Osaka Cancer Registry); S Sato, M Yoshii (Saga Prefectural Cancer Registry); I Oki (Tochigi Prefectural Cancer Registry); N Fukushima, A Shibata (Yamagata Prefectural Cancer Registry); K Iwasa, C Ono (Yamanashi Cancer Registry); T Matsuda* (National Cancer Center); Jordan: O Nimri (Jordan National Cancer Registry); Korea: K W Jung, Y J Won (Korea Central Cancer Registry); Kuwait: E Alawadhi, A Elbasmi (Kuwait Cancer Registry); Malaysia: A Ab Manan (Malaysia National Cancer Registry); F Adam (Penang Cancer Registry); Mongolia: E Sanjaajmats, U Tudev (Cancer Registry of Mongolia); C Ochir (Mongolian National University of Medical Sciences); Qatar: A M Al Khater, M M El Mistiri (Qatar Cancer Registry); Singapore: G H Lim, Y Y Teo (Singapore Cancer Registry); Taiwan: C J Chiang, W C Lee (Taiwan Cancer Registry); Thailand: R Buasom, S Sangrajrang (Bangkok Cancer Registry); S Kamsa-ard, S Wiangnon (Khon Kaen Provincial Cancer Registry); K Daoprasert, D Pongnikorn (Lampang Cancer Registry; Lamphun Cancer Registry); A Leklob,

S Sangkitipaiboon (Lopburi Cancer Registry); S L Geater, H Sriplung (Songkhla Cancer Registry); Turkey: O Ceylan, I Kög (Ankara Cancer Registry); O Dirican (Antalya Cancer Registry); T Köse (Bursa Cancer Registry); T Gurbuz (Edirne Cancer Registry); F E Karaşahin, D Turhan (Erzurum Cancer Registry Center); U Aktaş, Y Halat (Eskişehir Cancer Registry); S Eser, C I Yakut (Izmir Cancer Registry); M Altinisik, Y Cavusoglu (Samsun Cancer Registry); A Türkköylü, N Üçüncü (Trabzon Cancer Registry). Europe: Austria: M Hackl (Austrian National Cancer Registry); Belarus: A A Zborovskaya (Belarus Childhood Cancer Subregistry); O V Aleinikova (Belarusian Research Center for Pediatric Oncology, Hematology and Immunology); Belgium: K Henau, L Van Eycken (Belgian Cancer Registry); Bulgaria: Z Valerianova, M R Yordanova (Bulgarian National Cancer Registry); Croatia: M Šekerija (Croatian National Cancer Registry); Czech Republic: L Dušek, M Zvolský (Czech National Cancer Registry); Denmark: G Engholm, H Storm* (Danish Cancer Society); Estonia: K Innos, M Mägi (Estonian Cancer Registry); Finland: N Malila, K Seppä (Cancer Society of Finland); 
France: J Jégu, M Velten (Bas-Rhin General Cancer Registry); E Cornet, $\mathrm{X}$ Troussard (Registre Régional des Hémopathies Malignes de Basse Normandie); A M Bouvier (Registre Bourguignon des Cancers Digestifs); A V Guizard (Registre Général des Tumeurs du Calvados); V Bouvier, G Launoy (Registre des Tumeurs Digestives du Calvados) P Arveux (Breast cancers registry of Côte-d'Or France); M Maynadié, M Mounier (Hémopathies Malignes de Côte d'Or); A S Woronoff (Doubs and Belfort Territory General Cancer Registry); M Daoulas,

M Robaszkiewicz (Finistère Cancer Registry); J Clavel, S Goujon (French National Registry of Childhood Hematopoietic Malignancies); B Lacour (National Registry of Childhood Solid Tumors); I Baldi, C Pouchieu (Gironde Registry of Primary Central Nervous System Tumors); B Amadeo, G Coureau (General Cancer Registry of Gironde Department); A Monnereau (Registre des Hémopathies Malignes de la Gironde; French Network of Cancer Registries (FRANCIM)); S Orazio (Registre des Hémopathies Malignes de la Gironde); P M Preux, F Rharbaoui (Registre Général des Cancers de Haute-Vienne); E Marrer (Haut-Rhin Cancer Registry); B Trétarre (Registre des Tumeurs de l'Hérault); M Colonna, P Delafosse (Registre du Cancer du Département de l'Isère); K Ligier, S Plouvier (Registre Général des Cancers de Lille et de sa Region); A Cowppli-Bony, F Molinié (Loire-Atlantique-Vendée Cancer Registry); S Bara (Manche Cancer Registry); O Ganry, B LapôtreLedoux (Registre du Cancer de la Somme); P Grosclaude (Tarn Cancer Registry); N Bossard, Z Uhry (Hospices Civils de Lyon); F Bray*, M Piñeros* (International Agency for Research on Cancer); J Estève (Université Claude Bernard, Lyon); Germany: R Stabenow, $\mathrm{H}$ Wilsdorf-Köhler (Common Cancer Registry of the Federal States); A Eberle, S Luttmann (Bremen Cancer Registry); I Löhden, A L Nennecke (Hamburg Cancer Registry); J Kieschke, E Sirri (Epidemiological Cancer Registry of Lower Saxony); K Emrich, S R Zeissig (Rhineland Palatinate Cancer Registry); B Holleczek (Saarland Cancer Registry); N Eisemann, A Katalinic (Schleswig Holstein Cancer Registry); Gibraltar: R A Asquez, V Kumar (Gibraltar Cancer Registry); Greece: E Petridou (Nationwide Registry for Childhood Haematological Malignancies and Solid Tumors); Iceland: E J Ólafsdóttir, L Tryggvadóttir (Icelandic Cancer Registry, Icelandic Cancer Society); Ireland: K Clough-Gorr, P M Walsh (National Cancer Registry Ireland); H Sundseth* (European Institute of Women's Health); Italy: G Mazzoleni, F Vittadello (Registro Tumori Alto Adige); E Coviello, F Cuccaro (Registro Tumori Puglia-Sezione ASL BT); R Galasso (Registro Tumori di Basilicata); G Sampietro (Registro Tumori di Bergamo); A Giacomin $\uparrow$ (Piedmont Cancer Registry Provinces of Biella and Vercelli); M Magoni (Registro Tumori Dell'ASL Di Brescia); A Ardizzone (Registro Tumori Brindisi); A D’Argenzio (Caserta Cancer Registry); M Castaing, G Grosso (Integrated Cancer Registry of Catania-Messina-Siracusa-Enna); A M Lavecchia, A Sutera Sardo (Registro Tumori Catanzaro); G Gola (Registro Tumori della Provincia di Como); L Gatti, P Ricci (Registro Tumori Cremona; Registro Tumori Mantova); S Ferretti (Registro Tumori della Provincia di Ferrara); D Serraino, A Zucchetto (Registro Tumori del Friuli Venezia Giulia); M V Celesia, R A Filiberti (Registro Tumori Regione Liguria); F Pannozzo (Registro Tumori della Provincia di Latina); A Melcarne, F Quarta (Registro Tumori Della Provincia Di Lecce Sezione RTP); A G Russo (Registro Tumori Milano); G Carrozzi, C Cirilli (Registro Tumori della Provincia di Modena); L Cavalieri d'Oro, M Rognoni (Registro Tumori di Monza e Brianza); M Fusco, M F Vitale (Registro Tumori della ASL Napoli 3 Sud); M Usala (Nuoro Cancer Registry); R Cusimano, W Mazzucco (Registro Tumori di Palermo e Provincia); M Michiara, P Sgargi (Registro Tumori della Provincia di Parma); L Boschetti (Cancer Registry of the province of Pavia); E Borciani, P Seghini (Registro Tumori Piacenza); M M Maule, F Merletti (Piedmont Childhood Cancer Registry); R Tumino (Registro Tumori della Provincia di Ragusa); P Mancuso, M Vicentini (Registro Tumori Reggio Emilia); T Cassetti, R Sassatelli (Pancreas Tumour Registry of Reggio Emilia Province); F Falcini, S Giorgetti (Registro Tumori della Romagna); A L Caiazzo, R Cavallo (Registro Tumori Salerno); R Cesaraccio, D R Pirino (Registro Tumori della Provincia di Sassari); M L Contrino, F Tisano (Registro Tumori Siracusa); A C Fanetti, S Maspero (Registro Tumori della Provincia di Sondrio); S Carone, A Mincuzzi (Registro Tumori Taranto); G Candela, T Scuderi (Registro Tumori Trapani); M A Gentilini, S Piffer (Registro Tumori Trento); S Rosso (Piedmont
Cancer Registry); A Barchielli, A Caldarella (Registro Tumori della Regione Toscana); F Bianconi, F Stracci (Registro Tumori Umbro di Popolazione); P Contiero, G Tagliabue (Registro Tumori Lombardia, Provincia di Varese); M Rugge, M Zorzi (Registro Tumori Veneto); S Beggiato, A Brustolin (Registro Tumori Della Provincia Di Viterbo); F Berrino*, G Gatta, M Sant**(Fondazione IRCCS Istituto Nazionale dei Tumori); C Buzzoni, L Mangone (Italian Association of Cancer Registries [AIRTUM]); R Capocaccia*, R De Angelis (National Centre for Epidemiology); R Zanetti* (International Association of Cancer Registries; Piedmont Cancer Registry); Latvia: A Maurina, S Pildava (Latvian Cancer Registry); Lithuania: N Lipunova, I Vincerževskienė (Lithuanian Cancer Registry); Malta: D Agius, N Calleja (Malta National Cancer Registry); Netherlands: S Siesling, O Visser (Netherlands Cancer Registry, IKNL); Norway: S Larønningen, B Møller (The Cancer Registry of Norway); Poland: A Dyzmann-Sroka, M Trojanowski (Greater Poland Cancer Registry); S Góźdź, R Mężyk (Holy Cross Cancer Registry); T Mierzwa (Kuiavian-Pomeranian Cancer Registry); L Molong, J Rachtan (Lesser Poland Cancer Registry); S Szewczyk (Łódź Cancer Registry); J Błaszczyk, K Kępska (Lower Silesian Cancer Registry); B Kościańska (Lublin Cancer Registry); K Tarocińska (Lubush Cancer Registry); M Zwierko (Mazovian Cancer Registry); K Drosik (Opole Cancer Registry); K M Maksimowicz, E Purwin-Porowska (Podlahian Cancer Registry); E Reca, J Wójcik-Tomaszewska (Pomeranian Cancer Registry); A Tukiendorf (Silesian Cancer Registry); M Grądalska-Lampart, A U Radziszewska (Subcarpathian Cancer Registry); A Gos (Varmian-Mazurian Cancer Registry); M Talerczyk, M Wyborska (West-Pomeranian Cancer Registry); J A Didkowska, U Wojciechowska (National Cancer Registry); M Bielska-Lasota (National Institute of Public Health, NIH); Portugal: G Forjaz de Lacerda, R A Rego (Registo Oncológico Regional dos Açores); J Bastos, M A Silva (Registo Oncológico Regional do Centro); L Antunes, J Laranja Pontes (Registo Oncológico Regional do Norte); A Mayer-da-Silva, A Miranda (Registo Oncólogico Regional do Sul); Romania: L M Blaga, D Coza (Cancer Institute I. Chiricuta); Russia: M Y Valkov (Arkhangelsk Regional Cancer Registry); L Gusenkova, O Lazarevich (Population Cancer Registry of the Republic of Karelia); O Prudnikova, D M Vjushkov (Omsk Regional Cancer Registry); A G Egorova, A E Orlov (Samara Cancer Regional Registry); L A Kudyakov, L V Pikalova (Tomsk Regional Cancer Registry); Slovakia: J Adamcik, C Safaei Diba (National Cancer Registry of Slovakia); Slovenia: M Primic-Žakelj, V Zadnik (Cancer Registry of Republic of Slovenia); Spain: N Larrañaga, A Lopez de Munain (Basque Country Cancer Registry); A A Herrera, R Redondas (Registro Poblacional de Cáncer de la Comunidad Autónoma de Canarias); R Marcos-Gragera, M L Vilardell Gil (Epidemiology Unit and Girona Cancer Registry); E Molina, M J Sánchez Perez (Granada Cancer Registry); P Franch Sureda, M Ramos Montserrat (Mallorca Cancer Registry); M D Chirlaque, C Navarro (Murcia Cancer Registry); E E Ardanaz, M M Guevara (Registro de Cáncer de Navarra); R Fernández-Delgado, R Peris-Bonet (Registro Español de Tumores Infantiles); M Carulla, J Galceran (Tarragona Cancer Registry); C Alberich, M Vicente-Raneda (Comunitat Valenciana Childhood Cancer Registry); Sweden: S Khan, D Pettersson (Swedish Cancer Registry); P Dickman* (Karolinska Institutet, Stockholm); Switzerland: I Avelina, K Staehelin (Basel Cancer Registry); B Camey (Registre Fribourgeois des Tumeurs); C Bouchardy, R Schaffar (Geneva Cancer Registry); H Frick, C Herrmann (Cancer Registry Grisons and Glarus; Cancer Registry of St Gallen-Appenzell); J L Bulliard, M Maspoli-Conconi (Registre Neuchâtelois et Jurassien des Tumeurs); C E Kuehni, S M Redmond (Swiss Childhood Cancer Registry); A Bordoni, L Ortelli (Registro Tumori Canton Ticino); A Chiolero, I Konzelmann (Registre Valaisan des Tumeurs); K L Matthes, S Rohrmann (Cancer Registry Zürich and Zug); UK: J Broggio, J Rashbass (National Cancer Registration and Analysis Service England); D Fitzpatrick, A Gavin (Northern Ireland Cancer Registry); D I Clark, A J Deas (Scottish Cancer Registry); D W Huws, C White (Welsh Cancer Intelligence \& Surveillance Unit); C Allemani*, A Bonaventure, M P Coleman*, V Di Carlo, R Harewood, M Matz, L Montel, M Nikšić, B Rachet*, A D Turculeț (London School of Hygiene \& Tropical Medicine); R Stephens* (National Cancer Research Institute, London); C A Stiller* (Public Health England). Oceania: Australia: E Chalker, H Phung (Australian Capital Territory Cancer Registry); R Walton, H You (NSW Cancer Registry); S Guthridge, 
F Johnson (Northern Territory of Australia Cancer Registry); J Aitken, P Gordon (Queensland Cancer Registry); K D'Onise, K Priest (South Australian Cancer Registry); B C Stokes, A Venn (Tasmanian Cancer Registry); H Farrugia, V Thursfield (Victorian Cancer Registry); J Dowling (Western Australian Cancer Registry); D Currow* (Cancer Institute NSW); New Zealand: J Hendrix, C Lewis (New Zealand Cancer Registry). *CONCORD Steering Committee. $\uparrow$ A Giacomin passed away on March 23, 2017.

\section{Declaration of interests}

We declare no competing interests.

\section{Acknowledgments}

This work was funded by the Centers for Disease Control and Prevention (Atlanta, GA, USA); Swiss Re (London, UK); Swiss Cancer Research foundation (Bern, Switzerland); Swiss Cancer League (Bern, Switzerland); Institut National du Cancer (Paris, France); La Ligue Contre le Cancer (Paris, France); Rossy Family Foundation (Montreal, QC, Canada); National Cancer Institute (Bethesda, MD, USA); American Cancer Society (Atlanta, GA, USA); Susan G Komen Foundation (Dallas, TX, USA). We gratefully acknowledge the cancer registry personnel who have recorded the diagnosis and outcome for every patient with cancer in their jurisdictions over many years: without their efforts, we would know very little about trends in the global cancer burden or the effectiveness of health systems in addressing it. The protocol was translated into Arabic by Mufid El Mistiri (Qatar Cancer Registry, Doha, Qatar) and Eiman Alawadhi (London School of Hygiene \& Tropical Medicine [LSHTM], London, UK); into Chinese by Ning Wang, Shuo Liu (Beijing Cancer Registry, Beijing, China), Yannan Yuan (Beijing University Cancer Hospital, Beijing, China), and Chun-Ju Chiang (Taiwan Cancer Registry, Taipei, Taiwan); into French by Audrey Bonaventure and Michel Coleman (LSHTM); into Italian by Veronica Di Carlo, Cristina Renzi, and Claudia Allemani (LSHTM); into Japanese by Tomohiro Matsuda (National Cancer Centre, Tokyo, Japan), Mari Kajiwara, and Kayo Nakata (LSHTM); into Portuguese by Gulnar Azevedo e Silva (University of Rio de Janeiro State, Rio de Janeiro, Brazil); into Russian by Daria Dubovichenko and Mikhail Valkov (Arkhangelsk Cancer Registry, Arkhangelsk, Russia); and into Spanish by Gustavo Hernandez Suarez (National Cancer Institute, Bogotá, Colombia), Natalia Sanz (LSHTM), and Enrique Barrios (Cancer Registry of Uruguay, Montevideo, Uruguay). We are grateful for expert advice and ideas from many colleagues, including Marc Maynadié (Hémopathies Malignes de Côte d’Or, Dijon, France) for advice on the classification of haematological malignancies; Amy Kahn (New York State Cancer Registry, Albany, NY, USA), Ron Dewar (Cancer Care Nova Scotia, Halifax, NS, Canada) and Jennifer Stevens (National Cancer Institute, Bethesda, MD, USA) for the program to convert NAACCR data structures to meet the CONCORD protocol; Angela Mariotto (National Cancer Institute, Bethesda, MD, USA) for US mortality data, Reda Wilson (Centers for Disease Control and Prevention, Atlanta, GA, USA) for assistance with the National Program of Cancer Registries, and Giovanni Luca Lo Magno (Caltanissetta, Italy) for the program to convert Stata output into Microsoft Word files. We also thank Graciela Abriata (Instituto Nacional del Cáncer, Buenos Aires, Argentina); Cristian Herrera (Ministerio de Salud, Santiago, Chile); Daniel Salas Peraza (Ministerio de Salud, San José, Costa Rica); Noorlia Yahaya (Penang State Health Department, George Town, Malaysia); Niek Klazinga and Rie Fujisawa (OECD, Paris, France), and Steve Scoppa (Information Management Services, Calverton, MD, USA). We thank our LSHTM colleagues Natalia Sanz (CONCORD programme manager to March, 2017), Lisa Montel (CONCORD programme manager from April, 2017), Yuki Alencar (Cancer Survival Group coordinator), Adrian Turculet for maps and database management, and Hakim Miah for the CONCORD file transmission utility. We gratefully acknowledge endorsement of CONCORD by the following agencies: American Cancer Society (Atlanta, GA, USA); Asociación Española contra el Cáncer (Madrid, Spain); Association of European Cancer Leagues (Brussels, Belgium); Canadian Association of Provincial Cancer Agencies (Toronto, Canada); Canadian Council of Cancer Registries (Toronto, Canada); Childhood Cancer International (Nieuwegein, Netherlands); Children with Cancer UK (London, UK); Danish Cancer Society (Copenhagen, Denmark); European CanCer Organisation (Brussels, Belgium); European Cancer Patient Coalition (Brussels, Belgium); European Institute for
Women's Health (Dublin, Ireland); European Society for Medical Oncology (Lugano, Switzerland); Fondation de France (Paris, France); International Agency for Research on Cancer (Lyon, France); International Atomic Energy Agency (Vienna, Austria); International Network for Cancer Treatment and Research (Brussels, Belgium); International SOS (Papua, Indonesia); Israel Centre for Disease Control (Tel-Hashomer, Israel); Jolanta Kwaśniewska's Foundation (Warsaw, Poland); Liga Argentina de Lucha contra el Cancer (Buenos Aires, Argentina); Members of the European Parliament Against Cancer (Brussels, Belgium); National Cancer Institute Center for Global Health (Bethesda MD, USA); National Cancer Research Institute Consumer Liaison Group (Leeds, UK); National Institute for Cancer Epidemiology and Registration (Zürich, Switzerland); NCD Asia Pacific Alliance (Tokyo, Japan); North American Association of Central Cancer Registries (Springfield, IL, USA); OECD (Paris, France); Société Internationale d'Oncologie Pédiatrique (Geneva, Switzerland); Union for International Cancer Control (Geneva, Switzerland); WHO Regional Office for Europe (Copenhagen, Denmark); and World Bank (Washington, DC, USA). The interpretation of the findings in this report, and the opinions, conclusions, and recommendations are those of the authors and do not necessarily reflect the views or official position of the British Columbia Cancer Agency or Cancer Care Ontario (Canada); the Centers for Disease Control and Prevention, the National Cancer Institute, Maryland Cancer Registry, New Hampshire Department of Health and Human Services, New York City Department of Health and Mental Hygiene, Ohio Department of Health, Pennsylvania Department of Health, West Virginia Cancer Registry, or the Susan G Komen Foundation (USA); the Health Directorate of the Australian Capital Territory, or the Institut National du Cancer (France). We are very grateful to Giulia Vivaldi for expert assistance with preparation of this Article.

\section{References}

1 Schottenfeld D, Fraumeni JF, eds. Cancer epidemiology and prevention, 3rd edn. Oxford: Oxford University Press, 2006.

2 International Agency for Research on Cancer. GLOBOCAN 2012: estimated cancer incidence, mortality and prevalence worldwide in 2012. http://globocan.iarc.fr/Default.aspx (accessed Sept 25, 2017).

3 WHO. Cancer prevention and control in the context of an integrated approach: report by the Secretariat. Geneva: World Health Organization, 2016.

4 WHO. Cancer prevention and control [WHA 58.22]. Geneva: World Health Organization, 2017.

5 Vázquez TR, Ghebreyesus TA. Beating NCDs can help deliver universal health coverage. Lancet 2017; 390: 1473-74.

6 Allemani C, Weir HK, Carreira H, et al. Global surveillance of cancer survival 1995-2009: analysis of individual data for 25676887 patients from 279 population-based registries in 67 countries (CONCORD-2). Lancet 2015; 385: 977-1010.

7 Harlan LC, Warren JL. Global survival patterns: potential for cancer control. Lancet 2015; 385: 926-28.

8 Centers for Disease Control and Prevention. Cancer survival: the start of global surveillance. 2015. https://www.cdc.gov/cancer/ $\mathrm{dcpc} /$ research/articles/CONCORD-2.htm (accessed Nov 1, 2017).

9 Walters S, Benitez-Majano S, Muller P, et al. Is England closing the international gap in cancer survival? Br J Cancer 2015; 113: 848-60.

10 Independent Cancer Taskforce. Achieving world-class cancer outcomes: a strategy for England 2015-2020. London: NHS England, 2015.

11 International Atomic Energy Agency. Access to cancer therapy. Nov 20, 2015. https://www.youtube.com/watch?v=cXJ79_hxdNA (accessed Nov 1, 2017).

12 International Atomic Energy Agency. PACT's new campaign raises awareness of the persistent inequalities in access to lifesaving cancer services: PACT highlights the growing global divide in cancer survival rates. Sept 11, 2015. https://cancer.iaea.org/ newsstory.asp?id=167 (accessed Nov 1, 2017).

13 Weir HK, Stewart S, Allemani C, et al. Population-based cancer survival (2001-2009) in the United States: findings from the CONCORD-2 study. Cancer 2017; 123: 4963-68.

14 Allemani C, Coleman MP. Public health surveillance of cancer survival in the US and world-wide: the contribution of the CONCORD programme. Cancer 2017; 123: 4977-81. 
15 Ferlay J, Soerjomataram I, Dikshit R, et al. Cancer incidence and mortality worldwide: sources, methods and major patterns in GLOBOCAN 2012. Int J Cancer 2015; 136: E359-86.

16 UN Statistical Division. Standard country or area codes for statistical use (M49). 2015. https://unstats.un.org/unsd/ methodology/m49/ (accessed Sept 1, 2017).

17 Fritz AG, Percy C, Jack A, eds. International classification of diseases for oncology (ICD-O), 3rd edn. Geneva: World Health Organization, 2000.

18 Fritz AG, Percy C, Jack A, eds. International Classification of diseases for oncology (ICD-O), first revision of 3rd edn. Geneva: World Health Organization, 2013.

19 Sant M, Karjalainen-Lindsberg ML, Maynadié M, et al. Manual for coding and reporting haematological malignancies. Tumori 2010; 96: i-A32.

20 Steliarova-Foucher E, Stiller C, Lacour B, Kaatsch P. International classification of childhood cancer, 3rd edn. Cancer 2005; 103: 1457-67.

21 Matz M, Coleman MP, Sant M, et al. The histology of ovarian cancer: world-wide distribution and implications for international survival comparisons (CONCORD-2). Gynecol Oncol 2017; 144: 405-13.

22 Morton LM, Turner JJ, Cerhan JR, et al. Proposed classification of lymphoid neoplasms for epidemiologic research from the Pathology Working Group of the International Lymphom Epidemiology Consortium (InterLymph). Blood 2007; 110: 695-708.

23 Turner JJ, Morton LM, Linet MS, et al. InterLymph hierarchical classification of lymphoid neoplasms for epidemiologic research based on the WHO classification (2008): update and future directions. Blood 2010; 116: e90-98.

24 Woods LM, Rachet B, Ellis L, Coleman MP. Full dates (day, month, year) should be used in population-based cancer survival studies. Int J Cancer 2012; 131: E1120-24.

25 Surveillance Epidemiology and End Results program. Multiple primary and histology coding rules manual. Bethesda, MD: National Cancer Institute, 2013.

26 IARC Working Group. International rules for multiple primary cancers (ICD-O 3rd edn). Eur J Cancer Prev 2005; 14: 307-08.

27 Environmental Systems Research Institute. ArcGIS desktop: release 10.3. 2015. http://desktop.arcgis.com/en/arcmap/10.3/ main/get-started/whats-new-in-arcgis.htm (accessed June 1, 2017).

28 United Nations. World population prospects: the 2015 revision. Department of Economic and Social Affairs, Population Division, 2015. http://esa.un.org/unpd/wpp/ (accessed Jan 10, 2017).

29 Allemani C, Harewood R, Johnson C, et al. Population-based cancer survival in the US: data, quality control and statistical methods. Cancer 2017; 123: 4982-93.

30 Estève J, Benhamou E, Raymond L. Statistical methods in cancer research, volume IV. Descriptive epidemiology (IARC Scientific Publications no. 128). Lyon: International Agency for Research on Cancer, 1994.

31 Cutler SJ, Ederer F. Maximum utilisation of the life table method in analyzing survival. J Chronic Dis 1958; 8: 699-712.

32 Brenner $\mathrm{H}$, Gefeller O. An alternative approach to monitoring cancer patient survival. Cancer 1996; 78: 2004-10.

33 Brenner H, Spix C. Combining cohort and period methods for retrospective time trend analyses of long-term cancer patient survival rates. Br J Cancer 2003; 89: 1260-65.

34 Pohar Perme M, Stare J, Estève J. On estimation in relative survival. Biometrics 2012; 68: 113- 20.

35 Clerc-Urmès I, Grzebyk M, Hédelin G. Net survival estimation with stns. Stata J 2014; 14: 87-102.

36 Rachet B, Maringe C, Woods LM, Ellis L, Spika D, Allemani C. Multivariable flexible modelling for estimating complete, smoothed life tables for sub-national populations. BMC Public Health 2015; 15: 1240.

37 Micheli A, Baili P, Mugno E, et al. Life expectancy and cancer survival in the EUROCARE-3 cancer registry areas. Ann Onco 2003; 14 (suppl 5): 28-40.

38 Ewbank DC, Gómez de León JC, Stoto MA. A reducible four-parameter system of model life tables. Popul Studies 1983; 37: 105-29.
39 Brass W. On the scale of mortality. In: Brass W, ed. Biological aspects of demography. London: Taylor and Francis, 1971: 69-110.

40 Elandt-Johnson RC, Johnson NL. Survival models and data analysis (Wiley series in probability and mathematical statistics). Indianapolis, IN: John Wiley \& Sons, 1980.

41 Corazziari I, Quinn MJ, Capocaccia R. Standard cancer patient population for age standardising survival ratios. Eur J Cancer 2004; 40: $2307-16$

42 Stiller CA, Bunch KJ. Trends in survival for childhood cancer in Britain diagnosed 1971-85. Br J Cancer 1990; 62: 806-65.

43 Greenwood M. The natural duration of cancer. Report on public health and medical subjects no. 33. London: HM Stationery Office, 1926.

44 Berkson J, Gage RP. Survival curve for cancer patients following treatment. J Am Stat Assoc 1952; 47: 501-15.

45 Dickman PW, Adami HO. Interpreting trends in cancer patient survival. J Intern Med 2006; 260: 103-17.

46 Coleman MP. Cancer survival: global surveillance will stimulate health policy and improve equity. Lancet 2014; 383: 564-73.

47 Allemani C. The importance of global surveillance of cancer survival for cancer control: the CONCORD programme. Cancer Control (in press).

48 Abdel-Rahman MA, Stockton DL, Rachet B, Hakulinen T, Coleman MP. What if cancer survival in Britain were the same as in Europe: how many deaths are avoidable? Br J Cancer 2009; 101 (suppl 2): 115-24.

49 Ellis L, Coleman MP, Rachet B. How many deaths would be avoidable if socioeconomic inequalities in cancer survival in England were eliminated? A national population-based study, 1996-2006. Eur J Cancer 2012; 48: 270-78.

50 Ho GF, Taib NA, Pritam Singh RK, Yip CH, Abdullah MM, Lim TO. What if all patients with breast cancer in Malaysia have access to the best available care: how many deaths are avoidable? Glob J Health Sci 2017; 9: 32-39.

51 Pokhrel A, Martikainen P, Pukkala E, Rautalahti M, Seppä K, Hakulinen T. Education, survival and avoidable deaths in cancer patients in Finland. Br J Cancer 2010; 103: 1009-114.

52 Sandiford P, Abdel-Rahman ME, Allemani C, Coleman MP, Gala G How many cancer deaths could New Zealand avoid if five-year relative survival ratios were the same as in Australia? Aust NZ J Public Health 2015; 39: 157-61.

53 Seppä K, Hakulinen T, Läärä E. Avoidable deaths and random variation in patients' survival. Br J Cancer 2012; 106: 1846-49

54 Mirzaei M, Mirzadeh M, Mirzaei M. Expected years of life lost due to adult cancer mortality in Yazd (2004-2010). Asian Pac J Cancer Prev 2016; 17: 101-05.

55 Brustugun OT, Møller B, Helland Å. Years of life lost as a measure of cancer burden on a national level. Br J Cancer 2014; 111: 1014-20.

56 Soerjomataram I, Lortet-Tieulent J, Parkin DM, et al. Global burden of cancer in 2008: a systematic analysis of disability-adjusted life-years in 12 world regions. Lancet 2012; 380: 1840-50.

57 Woods LM, Morris M, Rachet B. No 'cure' within 12 years of diagnosis among breast cancer patients who are diagnosed via mammographic screening: women diagnosed in the West Midlands region of England 1989-2011. Ann Oncol 2016; 27: 2025-31

58 Andersson TML, Eriksson H, Hansson J, et al. Estimating the cure proportion of malignant melanoma, an alternative approach to assess long term survival: a population-based study. Cancer Epidemiol 2014; 38: 93-99.

59 Gatta G, Rossi S, Foschi R, et al. Survival and cure trends for European children, adolescents and young adults diagnosed with acute lymphoblastic leukemia from 1982 to 2002. Haematologica 2013; 98: 744-52.

60 Ito Y, Nakayama T, Tsukuma $\mathrm{H}$, et al. Role of age and tumour stage in the temporal pattern of 'cure' from stomach cancer: a population-based study in Osaka, Japan. Cancer Epidemiol 2012; 36: $128-32$.

61 Shack LG, Shah A, Lambert P, Rachet B. "Cure" by age and stage at diagnosis for colorectal cancer patients: a population-based study. Cancer Epidemiol 2012; 36: 548-53.

62 Coleman MP. Why we need global surveillance of cancer survival: the CONCORD programme. In: McGrath I, ed. Cancer care in emerging health systems. Oxford: International Network for Cancer Treatment and Research, 2013: 60-65. 
63 Cookson JB. Cancer survival. Lancet 2000; 356: 1611.

64 OECD. Cancer care: assuring quality to improve survival. Paris: Organisation for Economic Co-operation and Development, 2013.

65 Atun R, Jaffray DA, Barton MB, et al. Expanding global access to radiotherapy. Lancet Oncol 2015; 16: 1153-86.

66 Meara JG, Leather AJM, Hagander L, et al. Global Surgery 2030: evidence and solutions for achieving health, welfare, and economic development. Lancet 2015; 386: 569-624.

67 OECD. Health at a glance 2017: OECD indicators. Paris Organisation for Economic Co-operation and Development, 2017.

68 UICC. World Cancer Declaration 2013. Geneva: Union for International Cancer Control, 2013.

69 Jensen OM, Parkin DM, MacLennan R, Muir CS, Skeet RG, eds. Cancer registration: principles and methods (IARC Scientific Publications no. 95). Lyon: International Agency for Research on Cancer, 1991.

70 Berrino F, Estève J, Coleman MP. Basic issues in the estimation and comparison of cancer patient survival. In: Berrino F, Sant M, Verdecchia A, Capocaccia R, Hakulinen T, Estève J, eds. Survival of cancer patients in Europe: the EUROCARE study (IARC Scientific Publications no. 132). Lyon: International Agency for Research on Cancer (WHO), 1995: 1-14.

71 Abdel-Razeq H, Attiga F, Mansour A. Cancer care in Jordan. Hematol Oncol Stem Cell Ther 2015; 8: 64-70.

72 Laurenti R, Coleman MP, Aylin P. Accuracy of statements of the cause of death on death certificates and the international comparability of mortality statistics. In: Coleman MP, Aylin P, eds. Death certification and mortality statistics: an international perspective. London: Office for National Statistics, 2000: 1-9.

73 Ovcharov VK, Tishuk EA. The improvement of statistical records of the causes of death in Russia. In: Coleman MP, Aylin P, eds. Death certification and mortality statistics: an international perspective. London: Office for National Statistics, 2000: 28-30.

74 Filippatos G, Andriopoulos P, Panoutsopoulos G, et al. The quality of death certification practice in Greece. Hippokratia 2016; 20: 19-25.

75 Haghighi MH, Dehghani M, Teshizi SH, Mahmoodi H. Impact of documentation errors on accuracy of cause of death coding in an educational hospital in Southern Iran. HIM J 2014; 43: 35-42.

76 Cândida da Cunha C, Teixeira R, França E. Assessment of the investigation of ill-defined causes of death in Brazil in 2010 Epidemiol Serv Saude 2017; 26: 1-12.

77 Antoni S, Soerjomataram I, Møller B, Bray F, Ferlay J. An assessment of GLOBOCAN methods for deriving national estimates of cancer incidence. Bull World Health Organ 2016; 94: 174-84.

78 Engeland A, Haldorsen T, Dickman PW, et al. Relative survival of cancer patients: a comparison between Denmark and other Nordic countries. Acta Oncol 1998; 37: 49-59.

79 Berrino F, Capocaccia R, Estève J, et al, eds. Survival of cancer patients in Europe: the EUROCARE-2 study (IARC Scientific Publications no. 151). Lyon: International Agency for Research on Cancer, 1999.

80 Storm HH, Gislum M, Engholm G. Cancer survival before and after initiating the Danish Cancer Control plan. Ugeskr Lager 2008; 170: 3065-69 (in Danish).

81 Engholm G, Ferlay J, Christensen N, et al. NORDCAN: cancer incidence, mortality, prevalence and survival in the Nordic Countries, version 7.3 (08.07.2016). Association of the Nordic Cancer Registries, Danish Cancer Society, 2016. http://www.ancr.nu (accessed Nov 3, 2017).

82 Storm HH, Engholm G, Hakulinen T, et al. Survival of patients diagnosed with cancer in the Nordic countries up to 1999-2003 followed to the end of 2006. A critical overview of the results. Acta Oncol 2010; 49: 532-44.

83 Yoo KY. Cancer control activities in the Republic of Korea. Jpn J Clin Oncol 2008; 38: 327-33.

84 Choi KS, Jun JK, Suh M, et al. Effect of endoscopy screening on stage at gastric cancer diagnosis: results of the National Cancer Screening Programme in Korea. Br J Cancer 2015; 112: 608-12.

85 Pech O, May A, Manner H, et al. Long-term efficacy and safety of endoscopic resection for patients with mucosal adenocarcinoma of the esophagus. Gastroenterology 2014; 146: 652-60.
86 Suh YS, Yang HK. Screening and early detection of gastric cancer: East versus West. Surg Clin North Am 2015; 95: 1053-66.

87 Chang JW-C, Guo J, Hung C-Y, et al. Sunrise in melanoma management: time to focus on melanoma burden in Asia. Asia Pac J Clin Oncol 2017; 13: 423-27.

88 Brawley OW. Accepting the existence of breast cancer overdiagnosis. Ann Intern Med 2017; 166: 364-65.

89 Marcus PM, Prorok PC, Miller AB, DeVoto EJ, Kramer BS. Conceptualizing overdiagnosis in cancer screening. J Natl Cancer Inst 2015; 107: 1-4.

90 Allemani C, Rachet B, Weir HK, et al. Colorectal cancer survival in the USA and Europe: a CONCORD high-resolution study. BMJ Open 2013; 3: e003055.

91 Maringe C, Walters S, Rachet B, et al. Stage at diagnosis and colorectal cancer survival in six high-income countries: a population-based study of patients diagnosed during 2000-2007. Acta Oncol 2013; 52: 919-32.

92 Wu SJ, Chiang CJ, Lin CT, Tien HF, Lai MS. Improving but inferior survival in patients with chronic lymphocytic leukemia in Taiwan: a population-based study, 1990-2004. PLoS One 2013; 8: e62930.

93 Sullivan R, Kowalczyk JR, Agarwal B, et al. New policies to address the global burden of childhood cancers. Lancet Oncol 2013; 14: e125-35.

94 Magrath I, Steliarova-Foucher E, Epelman S, et al. Paediatric cancer in low-income and middle-income countries. Lancet Oncol 2013; 14: e104-16.

95 Mostert S, Arora RS, Arreola M, et al. Abandonment of treatment for childhood cancer: position statement of a SIOP PODC Working Group. Lancet Oncol 2011; 12: 719-20.

96 Pritchard-Jones K, Stiller CA. What can we learn from geographical comparisons of childhood cancer survival? Br J Cancer 2007; 96: 1493-97.

97 Ginsburg O, Bray F, Coleman MP, et al. The global burden of women's cancers: a grand challenge in global health. Lancet 2017; 389: 847-60.

98 Ginsburg O, Badwe R, Boyle P, et al. Changing global policy to deliver safe, equitable and affordable care for women's cancers. Lancet 2017; 389: 871-80.

99 Beaulieu N, Bloom D, Bloom R, Stein R. Breakaway: the global burden of cancer-challenges and opportunities. A report from the Economist Intelligence Unit. London: The Economist, 2009.

100 Mariotto AB, Yabroff KR, Shao Y, Feuer EJ, Brown ML. Projections of the cost of cancer care in the United States: 2010-2020. J Natl Cancer Inst 2011; 103: 117-28.

101 Fojo T, Grady C. How much is life worth: cetuximab, non-small cell lung cancer, and the $\$ 440$ billion question. J Natl Cancer Inst 2009; 101: $1044-48$.

102 Knaul FM, Gralow J, Atun R, Bhadelia A. Closing the cancer divide: an equity imperative. Boston, MA: Harvard University Press, 2011.

103 Sullivan R, Peppercorn J, Sikora K, et al. Delivering affordable cancer care in high-income countries. Lancet Oncol 2011; 12: 933-80.

104 Tangka FKL, Subramanian S, Beebe MC, et al. Cost of operating central cancer registries and factors that affect cost: findings from an economic evaluation of Centers for Disease Control and Prevention National Program of Cancer Registries. J Public Health Manag Pract 2016; 22: 452-60.

105 United Nations. Sustainable Development Goals. 2015. http://www.un.org/sustainabledevelopment/sustainabledevelopment-goals/ (accessed Sept 25, 2017).

106 United Nations. Sustainable Development Goal 3. 2015. https://sustainabledevelopment.un.org/sdg3 (accessed Sept 25, 2017).

107 Schmidt H, Gostin LO, Emanuel EJ. Public health, universal health coverage, and sustainable development goals: can they coexist? Lancet 2015; 386: 928-30.

108 López A, Thomason J. Civil registration and vital statisticseverybody's business but nobody's business. Lancet 2013; 381: $1275-76$

109 Setel PW, Macfarlane SB, Szreter S, et al. Who counts? 1: A scandal of invisibility: making everyone count by counting everyone. Lancet 2007; 370: 1569-77.

110 WHO. Cancer prevention and control in the context of an integrated approach [A70/A/CONF./9]. Geneva: World Health Organization, 2017. 